Chapter 1

\title{
Quaternary Geochronology Using Accelerator Mass Spectrometry (AMS) - Current Status of the AMS System at the TONO Geoscience Center
}

\author{
Akihiro Matsubara, Yoko Saito-Kokubu, \\ Akimitsu Nishizawa, Masayasu Miyake, \\ Tsuneari Ishimaru and Koji Umeda \\ Additional information is available at the end of the chapter \\ http://dx.doi.org/10.5772/58549
}

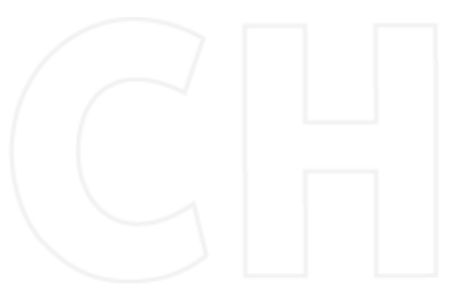

\section{Introduction}

\subsection{Background}

The Tono Geoscience Center (TGC) of the Japan Atomic Energy Agency (JAEA) has been conducting research into the long term (several million years) stability of underground environments, in order to provide the scientific knowledge needed to ensure safety and reliability for the geological disposal of high-level radioactive waste [1-3]. The time scale for occurrence of the relevant geoscientific activities, as shown in Figure 1, i.e., earthquake/fault and volcanic activities, behavior of groundwater flow, uplift/subsidence and erosion of the ground surface, and so on, corresponds well to the duration of the Quaternary Period geology. Geochronology of the Quaternary Period has been strongly enhanced by measurement of terrestrial in situ cosmogenic radionuclides, such as ${ }^{10} \mathrm{Be},{ }^{14} \mathrm{C},{ }^{26} \mathrm{Al}$, and ${ }^{36} \mathrm{Cl}$, produced by secondary cosmic rays (e.g., neutron, muon) which are generated by interaction between the atmosphere of earth and primary cosmic rays that originate from the sun and galactic systems.

Applications of accelerator mass spectrometry (AMS) using those rare radionuclides for geological studies have been summarized by various authors [4-7]. It is a well-known fact that ${ }^{14} \mathrm{C}$ has been widely utilized in several disciplines, including geology, environmental science, archaeology, and biomedicine. With regard to research into underground geological disposal of waste, radiocarbon dating of organic samples (e.g., bulk organic, humic acid, and humin fractions) taken from faults provide an historical archive of typical conventional applications 
to the investigation of seismic activity $[7,8]$. The dates obtained, combined with other scientific and historical information, help to determine whether or not the fault is a so called "active fault", and to estimate cyclicity of seismic events and probability for serious large fault movements during the post-closure duration of geological disposal.

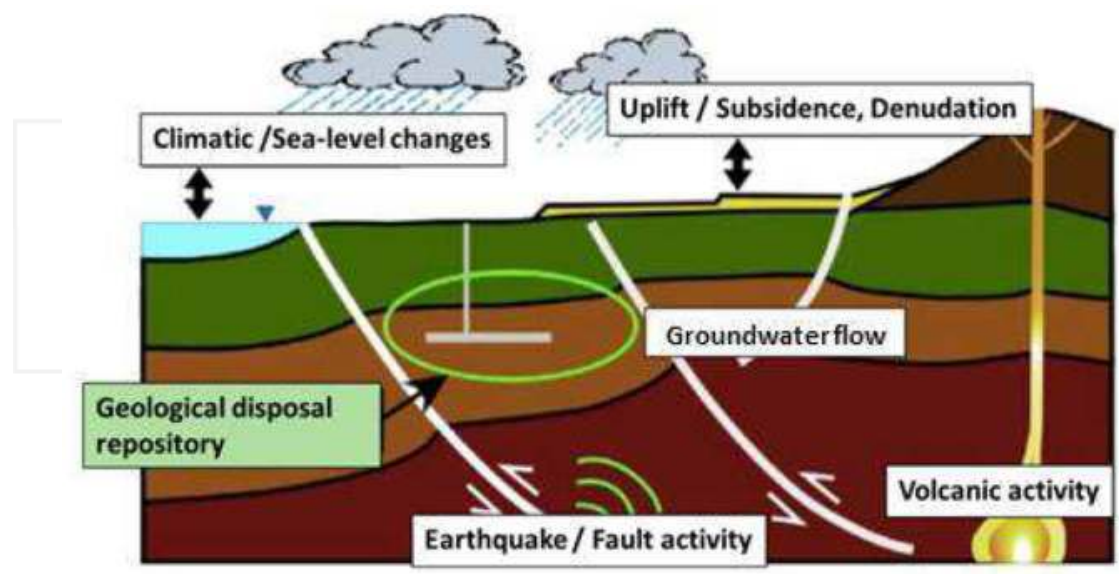

Figure 1. Long-term geological activities relevant to the geological disposal of high-level radioactive waste.

Long-lived cosmogenic radionuclides, such as ${ }^{10} \mathrm{Be},{ }^{26} \mathrm{Al}$, and ${ }^{36} \mathrm{Cl}$ enable us to apply to the exposure dating methods on boulder and bedrock surfaces for exposure ages up to $10^{7}$ years [4-7]. These methods can provide information relevant to geological disposal with respect to geomorphological evolution, i.e., erosion rates of rock surfaces, burial histories of rock surfaces and sediments, fault slip rates, and so on. One of the typical radionuclides for surface exposure dating is ${ }^{10} \mathrm{Be}$, or both ${ }^{10} \mathrm{Be}$ and ${ }^{26} \mathrm{Al}$. The half-lives of ${ }^{10} \mathrm{Be}$ and ${ }^{26} \mathrm{Al}$ are long enough $(1.6 \mathrm{Myr}$ and $0.7 \mathrm{Myr}$, respectively) to span the entire Quaternary timescale. They can be produced simultaneously in a single sample of quartz where ${ }^{26} \mathrm{Al}$ and ${ }^{10} \mathrm{Be}$ are mainly produced through nuclear spallation from ${ }^{28} \mathrm{Si}$ and ${ }^{16} \mathrm{O}$, respectively. Concentration of each depends on balance between in situ production and surface erosion and generally, there are two unknown variables. The simultaneous measurement of ${ }^{26} \mathrm{Al}$ and ${ }^{10} \mathrm{Be}$ concentration has the advantage of solving for the two variables: exposure age and erosion rate. On the other hand, ${ }^{36} \mathrm{Cl}$ is produced through multi-channel reactions: spallation on $\mathrm{Ca}$ and $\mathrm{K}$ with neutron and muon, and the thermal neutron capture on ${ }^{35} \mathrm{Cl}$. This feature provides an advantage to the ${ }^{36} \mathrm{Cl}$ exposure dating that is not restricted to specific rock types or minerals (such as carbonates or silicates) under a number of conditions. Furthermore, decomposition of contribution for ${ }^{36} \mathrm{Cl}$ production into spallogenic, mugenic, and thermal neutron can increase the amount of information for one sample, with the potential for greater erosion-exposure history accuracy.

The measurement of ${ }^{14} \mathrm{C}$ and ${ }^{36} \mathrm{Cl}$ is also applicable to hydrogeologic investigations: studies of groundwater age, origin and mixing. Most of these nuclides are produced through interaction 
with alpha-particle/neutron emitted from radioactive elements such as Th and $U$ within the sediment or rock dozens of meters or more underground, where there is no cosmogenic radionuclide production [5].

\subsection{Purpose and contents of this article}

Our ongoing efforts, therefore, have been dedicated to development of a multi-nuclide AMS for measurement of the rare radionuclides ${ }^{10} \mathrm{Be},{ }^{14} \mathrm{C},{ }^{26} \mathrm{Al}$, and ${ }^{36} \mathrm{Cl}$. In this article, the current status of the AMS system at the JAEA-AMS-TONO and our activities leading to development of a multi-nuclide AMS are presented.

The next section shows the history and present-day status of our AMS system. The detail of the AMS system and its configuration are described in Section 3. The current status for ${ }^{14} \mathrm{C}$, ${ }^{10} \mathrm{Be}$ and ${ }^{26} \mathrm{Al}$ measurements is presented in Sections 4, 5, and 6, respectively. Section 7 provides the research and development related to improvement of the isobar discrimination for the ionization chamber. Finally, Section 8 presents a summary.

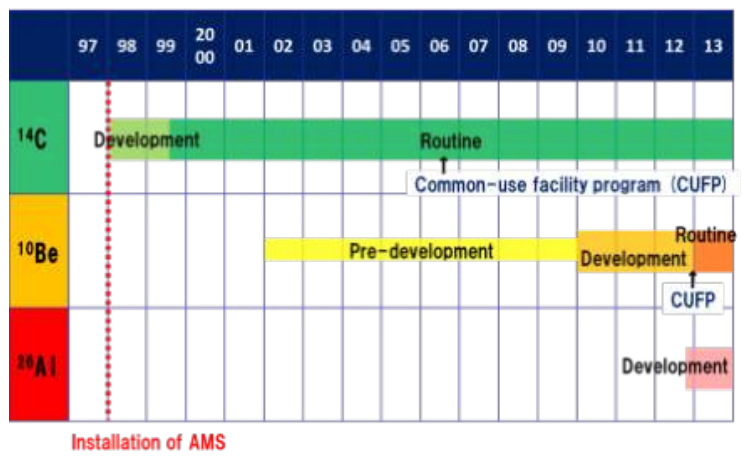

Figure 2. Timeline of the JAEA-AMS-TONO development.

\section{Operation status}

The history of the JAEA AMS system is depicted in Figure 2. The AMS system was installed at TGC in 1997, and routine measurement of ${ }^{14} \mathrm{C}$ started in 1998 [9]. The preliminary development of the ${ }^{10} \mathrm{Be}-\mathrm{AMS}$ started around 2002 [10], intensive development was implemented from 2010 to 2012 [11,12], and progress of which will be described in Section 5. After that, the routine measurement of ${ }^{10} \mathrm{Be}$ started at the beginning of fiscal year 2013. At present, we have initiated the development of the ${ }^{26} \mathrm{Al}$-AMS (described in Section 6) [13, 14]. Furthermore, as a part of preparatory activity for the development of the ${ }^{36} \mathrm{Cl}$-AMS, we have started to investigate the nature of the pulse trace that is disturbed by interfering particles in the heavy ion detector, in order to improve the discrimination performance for the detector system (presented in Section 7) [15]. 
The evolutions of the measurement time and the number of sample cathode (target) are shown in Figure 3. Total, cumulative, measurement time (the blue line) has increased more or less continuously for 15 years, and reached 15,000 hours this fiscal year. Around 2005, routine measurements ceased for a while due to system maintenance by the lab-staff. As shown on the bar chart, the average number of samples measured annually is between 800 and 1000, and the total number of samples will exceed 12,500 within the next few months. After the development of the ${ }^{10} \mathrm{Be}$ measurement has been intensive since the start, the proportion of ${ }^{10} \mathrm{Be}$ samples to the total sample number has increased rapidly. In fiscal year 2012, the proportion of ${ }^{14} \mathrm{C},{ }^{10} \mathrm{Be}$ and ${ }^{26} \mathrm{Al}$ sample cathodes are $76 \%, 20 \%$, and $4 \%$, respectively.

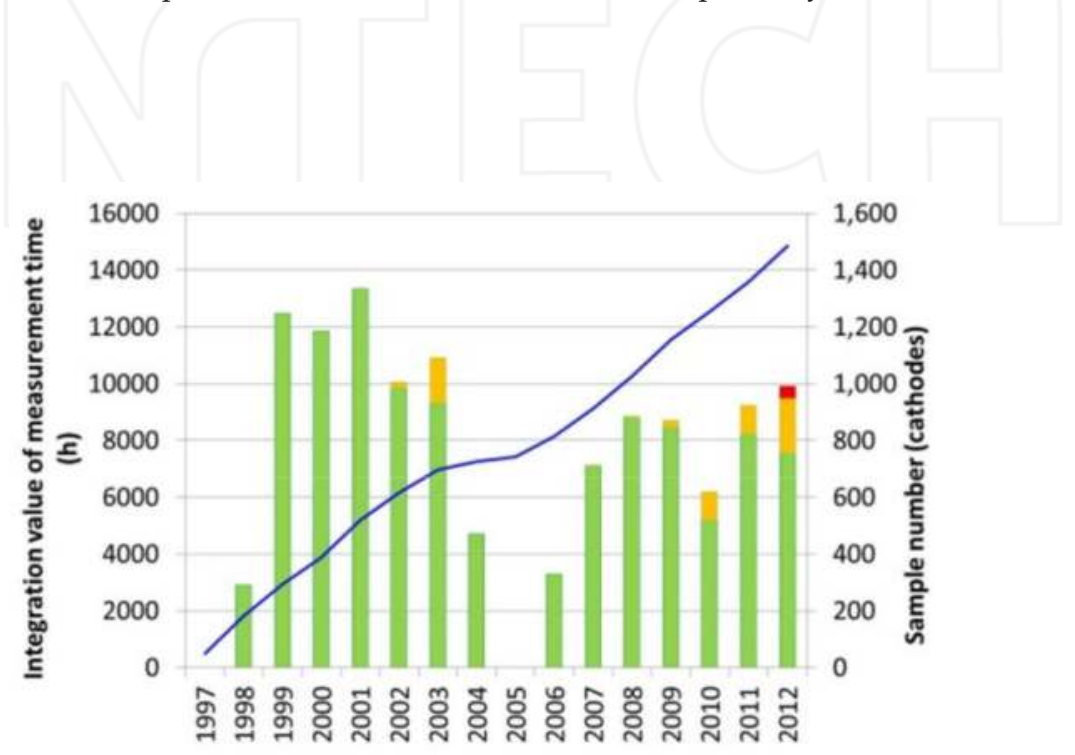

Annual measurement time

Figure 3. Evolution of the measurement time and sample number.

In Figure 4, the pie chart on the left shows the proportion of ${ }^{14} \mathrm{C}$-AMS samples measured for the various study fields in fiscal year 2012. Geoscience accounted for about $60 \%$, while environmental studies accounted for most of the balance. The proportion labeled as "Analysis" stands for the cathode number used in our technical development. The pie chart on the right in Figure 4 illustrates the relative proportion of measured samples requested by users in JAEA to other users. Almost all of the samples were requested by JAEA users. The measurement of the other samples were performed under JAEA's common-use facility program for non-JAEA users [16]. This program started in 2006, in order to enlarge and expand the public use of JAEA's facilities. The study fields using the program were mostly in environmental science and archaeology. 
Figure 4. Proportion of ${ }^{14} \mathrm{C}$-AMS samples measured in the various investigation fields (left) and, on the right, those requested by JAEA users to by others in fiscal year 2012.

\section{AMS system}

\subsection{Overall features}

The AMS system is a versatile system based on the Pelletron ${ }^{\mathrm{TM}}$ tandem accelerator (Model 15SDH-2, 5 MV terminal voltage) [17]. The same type (5 MV Pelletron) of the AMS system has been used in other facilities, for example, at the Micro Analysis Laboratory, Tandem accelerator (MALT) at the University of Tokyo, Japan [18], the AMS system at the National Institute for Environmental Studies (NIES) of Japan [19], at the Scottish Universities Environmental Research Center (SUERC) in the United Kingdom [20,21], and at the Uppsala 5 MV Pelletron tandem accelerator developed in the Uppsala University, Sweden [22,23].

This AMS system is designed for the AMS analysis with most radio-isotopes including ${ }^{10} \mathrm{Be}$, ${ }^{14} \mathrm{C},{ }^{26} \mathrm{Al},{ }^{36} \mathrm{Cl}$, and ${ }^{129} \mathrm{I}$. Although technological advances in recent years have enabled practical use of compact AMS systems below $1 \mathrm{MV}$ allowing the measurement of ${ }^{10} \mathrm{Be},{ }^{14} \mathrm{C}$, and ${ }^{26} \mathrm{Al}[24-$ 26], the relatively wide range of high terminal voltage greater than several megavolts has, even now, been generally recognized to be beneficial to efficient suppression of signal background, resulting in further potential for expandability for a multi-nuclides measurement.

\subsection{System description [17]}

Figure 5 is a schematic of the AMS system layout. The system can be divided into five major subsystems: the ion sources, the sequential injection system, the tandem Pelletron accelerator, the post-accelerator beamline with the high-energy mass spectrometer components, and the heavy ion detection system by means of the ionization chamber. There are eight vacuum turbomolecular pumping systems attached along the beamline, where, several beam steerers 
and magnetic or electrostatic lenses are located, and the total length of the system is around 31 metres. Summary of the system configuration for rare isotopes are presented individually in Table 1.

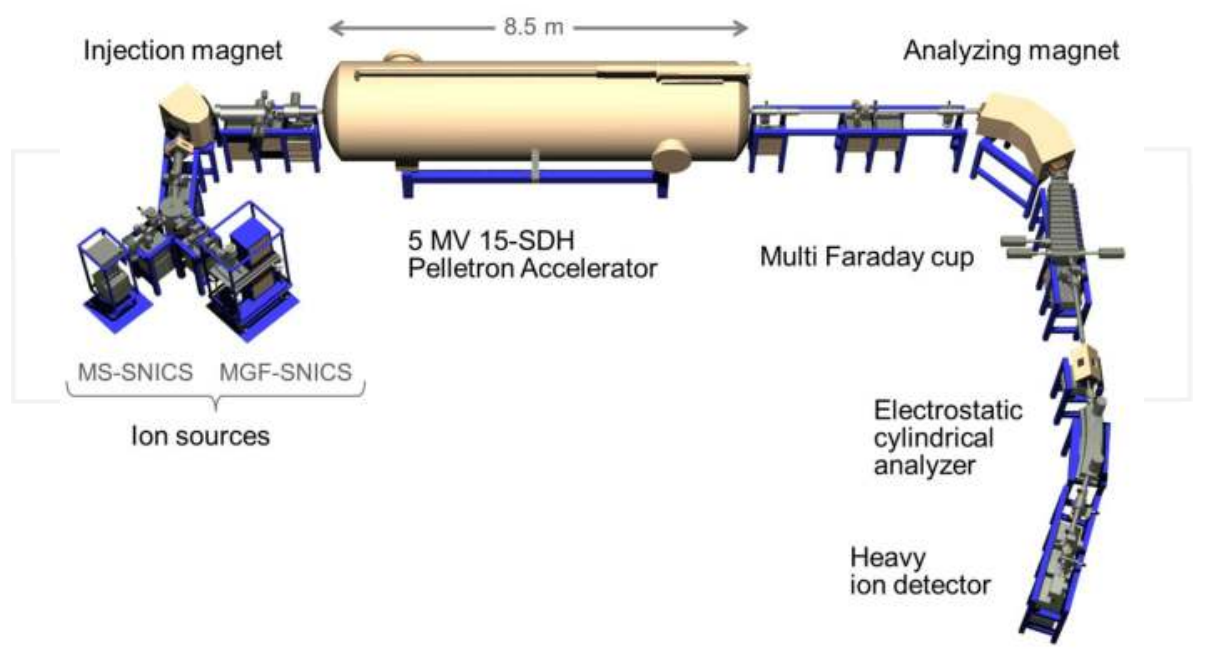

Figure 5. AMS system at JAEA-AMS-TONO.

\begin{tabular}{|c|c|c|c|}
\hline Nuclide & ${ }^{14} \mathrm{C}$ & ${ }^{10} \mathrm{Be}$ & ${ }^{26} \mathrm{Al}$ \\
\hline $\begin{array}{l}\text { Terminal volt. } \\
\text { (Tot. Energy) }\end{array}$ & $\begin{array}{c}4.5 \mathrm{MV} \\
(22.5 \mathrm{MeV})\end{array}$ & $\begin{array}{c}4.8 \mathrm{MV} \\
(16.3 \mathrm{MeV})\end{array}$ & $\begin{array}{c}4.3 \mathrm{MV} \\
(17.2 \mathrm{MeV})\end{array}$ \\
\hline Target & $\begin{array}{l}\text { Graphite with Fe } \\
\text { powder }\end{array}$ & $\begin{array}{c}\text { BeO with } \mathrm{Nb} \\
\text { powder }\end{array}$ & $\begin{array}{l}\mathrm{Al}_{2} \mathrm{O}_{3} \text { with } \mathrm{Ag} \\
\text { powder }\end{array}$ \\
\hline Current & $20 \mu \mathrm{A}\left(\mathrm{C}^{-}\right)$ & $2 \mu \mathrm{A}\left(\mathrm{BeO}^{-}\right)$ & $0.1 \mu \mathrm{A}\left(\mathrm{Al}^{-}\right)$ \\
\hline Injection & $\begin{array}{l}\text { Sequential }\left({ }^{12} \mathrm{C}: 0.3 \mathrm{~ms},\right. \\
\left.{ }^{13} \mathrm{C}: 0.9 \mathrm{~ms},{ }^{14} \mathrm{C}: 98.6 \mathrm{~ms}\right)\end{array}$ & $\begin{array}{c}\text { Simultaneous } \\
\left({ }^{10} \mathrm{Be}^{16} \mathrm{O},{ }^{9} \mathrm{Be}^{17} \mathrm{O}\right)\end{array}$ & $\begin{array}{c}\text { Sequential } \\
\left({ }^{26} \mathrm{Al}: 98 \mathrm{~ms},{ }^{27} \mathrm{Al}: 1 \mathrm{~ms}\right)\end{array}$ \\
\hline Transmit. & $58 \%\left({ }^{12} \mathrm{C}\right)$ & $21 \%\left({ }^{9} \mathrm{Be}\right)$ & $39 \%\left({ }^{27} \mathrm{Al}\right)$ \\
\hline $\begin{array}{l}\text { Meas. ratio } \\
\text { (Count rate) }\end{array}$ & $\begin{array}{c}{ }^{14} \mathrm{C}^{4+} /{ }^{12} \mathrm{C}^{4+},{ }^{13} \mathrm{C}^{4+} /{ }^{12} \mathrm{C}^{4+} \\
(60 \mathrm{cps} @ \mathrm{HOx} \mathrm{II})\end{array}$ & $\begin{array}{l}{ }^{10} \mathrm{Be}^{3+} /{ }^{17} \mathrm{O}^{5+} \\
(70 \mathrm{cps} @ \mathrm{~S} 5-1)\end{array}$ & $\begin{array}{l}{ }^{26} \mathrm{Al}^{3+} /{ }^{27} \mathrm{Al}{ }^{3+} \\
(15 \mathrm{cps} @ S 4-1)\end{array}$ \\
\hline Background & $\begin{array}{c}<7 \times 10^{-16}(<0.06 \mathrm{pMC}) @ \text { WAKO } \\
\text { Powder }\end{array}$ & $\begin{array}{l}<7 \times 10^{-15} \\
\text { @MITSUWA powder }\end{array}$ & $\begin{array}{l}<3 \times 10^{-14} \\
@ B l a n k \dagger\end{array}$ \\
\hline Ionization chamber & $\Delta E_{1}-E_{\mathrm{Res}}$ & $\begin{array}{c}\Delta E_{1}-E_{\text {Res }} \\
\text { (with gas cell) }\end{array}$ & $\Delta E_{1}-E_{\mathrm{Tot}}$ \\
\hline
\end{tabular}

† Sample of BLK was made from a quantified standard sample for atomic absorption spectrometry supplied from Wako Pure Chemical Industries, Ltd.

Table 1. Summary of current AMS specifications for the JAEA-AMS-TONO. 
The ion sources, the Multi-Cathode, Source of Negative Ions by Cesium Sputtering (MCSNICS) for solid samples (40 cathodes) and the Multiple Gas Feed, SNICS (MGF-SNICS) for $\mathrm{CO}_{2}$-gas samples (12 cathodes) are connected to the main beamline through the $45^{\circ}$ electrostatic spherical analyzer (ESA). The sources consist of a cesium oven generating Cs vapour, a heated ionizer electrode producing a focused $\mathrm{Cs}^{+}$beam at the sample cathode, and extraction and focus electrodes. Particles sputtered from the sample cathode by $\mathrm{Cs}^{+}$bombardments pick up electrons as they pass through the cesium layer condensed on the sample; thus, negative ions are produced. To stabilize the cesium vapour feed to the source, we added a simple autocontrollable electrical heating subsystem to the cesium oven and its feeder pipe; the standard deviation of the temperature monitored during the routine measurement has been kept within a range of $\pm 0.5 \mathrm{C}^{\circ}$ [27]. This type of simple technical addition or modification is commonly used for the same purpose [28]. The acceleration voltage of the ion source is usually set to $55 \mathrm{kV}$. By using the beam-slit located at the image point of the ESA (before the injection magnet), the "tail" of the beam profile can be trimmed, where the tail is due to an energy spread in the sputtering process. This trimming assures open-aperture optical properties (often called "flat top transmission") on the downstream side of that slit. The combination of the ESA and the injection magnet ( $M E / q^{2} \leq 15$, where $M$ is the mass number of the ion, $E$ is the ion energy in $\mathrm{MeV}$ and $q$ is the charge number, and the nominal radius is $0.457 \mathrm{~m}$ ) limits the specific chargeto-mass ratio, $M / q$, for the transmittance of negative ions in the pre-accelerator region to remove unwanted particles from the ion beam. The mass resolution $(M / \Delta M)$ reaches 200 for the electrostatic and magnetic filters combination. The same concept is applied for the highenergy post-accelerator region containing the analyzing magnet and electrostatic cylindrical analyzer (ECA) listed below.

The AMS system employs the sequential injection method for the precise measurement of the ratio of rare to abundant isotopes regardless of fluctuations of source conditions. This method, or the rapid switching of the masses (isotopes) to be injected toward the accelerator, (so called sequence "bounced" or "jumping" beams) is accomplished by applying an appropriate bias potential to the electrically insulated bent chamber inside the injection magnet. Most of the duration ( 99\%) in the sequential injection is allocated for the measurement of the rare isotope (details in Table 1).

In the tandem Pelletron accelerator (15-SDH), there are two parallel chains charging the highvoltage terminal with current up to $300 \mu \mathrm{A}$. The consequent maximum terminal potential of $5 \mathrm{MV}$ leads to the suitable stripped ionization state of $4+$ for carbon by using a gas stripper (the ion beam energy is up to $25 \mathrm{MeV}$ ). For the chlorine, the charge state is designed to be $7+$ or $8+$ by using a foil stripper and its energy would be lie in the range of $42-45 \mathrm{MeV}$. The pressure of the stripper gas is typically $10 \mu$ Torr, $9 \mu$ Torr, and $5 \mu$ Torr for ${ }^{14} \mathrm{C}-,{ }^{10} \mathrm{Be}-$-, and ${ }^{26} \mathrm{Al}$-measurement, respectively.

The high-energy mass spectrometer in the post-accelerator region is composed of magnetic and electrostatic filters and detector systems. The analyzing magnet (produced by Danfysik $\mathrm{A} / \mathrm{S}$ ) is a double focusing $90^{\circ}$ sector magnet with a nominal radius of $1.270 \mathrm{~m}$, having parameters of $M E / q^{2} \leq 176$ and $M / \triangle M=725$. After the off-axis, multi-Faraday cup detector for the abundant isotopes, the $20^{\circ}$ ECA with a nominal radius of $3.810 \mathrm{~m}$ is used to remove abundant 
isotopes having the same mass energy product as the rare isotopes. The resolution attained by the ECA is $M / \triangle M=200$.

The final detector for counting the rare isotopes, the "heavy ion detector", is the gas ionization detector that contains multiple $\Delta E$ electrodes (five) arranged along the axis of the beamline (for details see Section 5.2). A nearly equivalent type of detector is used at the SUERC facility in the UK [21]. The $\Delta E$ electrodes determine the specific of energy loss arising in their respective regions. In the case for the ion energy over $\mathrm{MeV} / \mathrm{u}$, the rate of the energy loss (or stopping power) increases with increasing atomic number. Thus, the spectrum on the plane given by energy-loss 2D-coordinates for various combinations of $\Delta E$ electrodes shows its characteristic position on that plane, which makes it possible to distinguish the spectrum of desired nuclide from other spectra of interfering particles. Typical particles interfering with the rare isotopes of ${ }^{10} \mathrm{Be},{ }^{14} \mathrm{C}$, and ${ }^{36} \mathrm{Cl}$ are respectively ${ }^{10} \mathrm{~B},{ }^{7} \mathrm{Li}$, and ${ }^{36} \mathrm{~S}$; their $\mathrm{M} / q$ ratios can be equivalent to those of the rare isotop es, thus allowing their entry into the detector. The problem with respect to the ${ }^{10} \mathrm{~B}$ and ${ }^{36} \mathrm{~S}$ is known as an isobar problem (different elements but same atomic weight). The $M / q$ ratio of ${ }^{14} \mathrm{C}^{4+}$ coincides with that of ${ }^{7} \mathrm{Li}^{2+}$; although the prime number of charge state (3+ for ${ }^{14} \mathrm{C}$ in this case) is preferable to avoid the coincidence of the $M / q$ ratio, we focus on the higher stripping yield for $4+(59 \%$ at $4.5 \mathrm{MV}$ [5]) rather than $3+(55 \%$ at $2.9 \mathrm{MV}$ [5]). Since the relative difference between atomic numbers for the case of "Be and $\mathrm{B}$ " or " $\mathrm{Cl}$ and $\mathrm{S}$ " is much smaller than the difference for $\mathrm{C}$ and $\mathrm{Li}$, distinguishing them in the former case is harder than in the latter case. Therefore, discrimination techniques are crucial for measurements of ${ }^{10} \mathrm{Be}$ and ${ }^{36} \mathrm{Cl}$. A gas absorber technique has been employed in the ${ }^{10} \mathrm{Be}-\mathrm{AMS}$ operation, which is described in Section 5.2. In addition, investigation of a pulse trace fluctuation caused by interfering particles in a preparation for the ${ }^{36} \mathrm{Cl}$ measurement is presented in Section 7.

\section{4. ${ }^{14} \mathrm{C}$ measurement}

\subsection{Stability and reliability}

In the ${ }^{14} \mathrm{C}$-AMS operation, the stability and reliability of the routine measurements have been checked continuously against measuring standards. The typical standards are, IAEAC1, -C5, and -C6 [29], and the oxalic acid HOxII (SRM-4990C) that is produced by the National Institute of Standards and Technology, NIST in the USA. Such checks have been performed simultaneously with routine measurements. In our AMS analysis, usually only the HOxII is used for obtaining the normalization constant that is given by the $\delta^{13} \mathrm{C}$ corrected activity divided by the fraction modern for the HOxII (1.3406). Here, the term fraction modern is a quantity defined as the ratio of a sample ${ }^{14} \mathrm{C}$ activity $A_{\mathrm{SN}}$ to a normalized sample ${ }^{14} \mathrm{C}$ activity $A_{\mathrm{ON}}$, where $A_{\mathrm{ON}}$ is equal to the ${ }^{14} \mathrm{C}$-decay corrected absolute international standard specific activity $A_{\text {abs }}$ that is intended to correspond to the hypothetical specific activity of atmospheric carbon of year 1950 (in detail see Ref.[30,31]). With respect to data quality of the HOxII standard, therefore, only the relative precision (or relative standard deviation, rsd) affects the normalization constant; in other words, the accuracy itself has no meaning. The value of $r s d$ for the HOxII is around $0.22 \%$ in every routine-measurement. The 
procedure of data analysis, as well as ${ }^{10} \mathrm{Be}$ and ${ }^{26} \mathrm{Al}$ analyses has followed an algorithm in the software code "abc" available from the NEC Corporation [30]. It is noted that to obtain the net activity the ${ }^{14} \mathrm{C} /{ }^{12} \mathrm{C}$ ratio for all samples (except $\mathrm{C} 1$ ) is normally subtracted by the IAEA-C1 value as the chemical background due to the sample preparation, where $\mathrm{C} 1$ is made from marble, and its nominal value in the percent Modern Carbon (pMC) that is equivalent to the $100 \% \times$ fraction modern (described above) is $0.00 \pm 0.02$ [29].

The left column of rectangles on Figure 6 shows the evolution of pMC for IAEA-C6, -C5, and $-\mathrm{C} 1$ in the year 2013. In some periods no ${ }^{14} \mathrm{C}$ measurements were performed. The period from May to June was allotted for ${ }^{10} \mathrm{Be}$ measurements. Intensive system maintenance was carried out (normally annually) in August, and then ${ }^{10} \mathrm{Be}$ measurements were performed until the end of September. Almost all measured pMC-values for both C6 and C5 are in agreement with the nominal values within $3 \sigma$ of each point ( $\sigma$ is basically the statistical uncertainty that is inversely proportional to the square root of ${ }^{14} \mathrm{C}$ counts). A few irregular points in $\mathrm{C} 6$ could be due to surface roughness of the graphite sample. The roughness is reflected by unsuccessful graphite compression with an Arbor press (hammering with a press-pin). We continue to check the surface condition and data related to such irregular results.
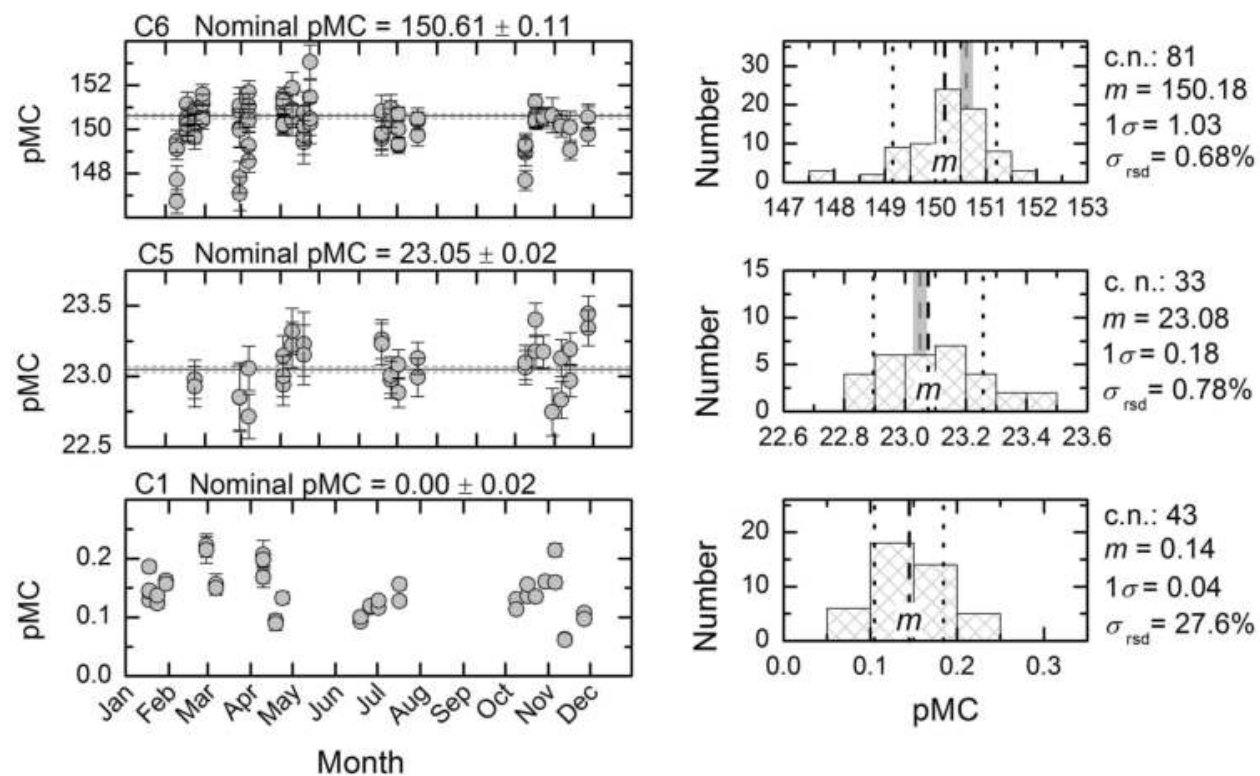

Figure 6. Measurement quality in a 12-month period for ${ }^{14} \mathrm{C}$-AMS standards: C6 (a), C5 (b), and C1 (c). The left column shows evolutions of the measured value in percent Modern Carbon for the year 2013. The gray line and hatch for both left and right columns depicts respectively the nominal value and its range of uncertainty. The right column shows corresponding histograms for the left column measurement points. The dashed and dotted lines present respectively the arithmetic mean $m$ and its standard deviation $\sigma_{H}$ on the histogram. The symbol c.n. stands for the number of sample cathodes used in constructing the histogram, and $\sigma_{\mathrm{Hrsd}}$ is the relative standard deviation given by $\sigma_{\mathrm{H}} / m \times 100 \%$. 
The frames in the right-hand side of Figure 6 show histograms corresponding to temporal evolution for each standard in the left frames. It can be seen that the arithmetic mean of the histogram for C6 (labelled as $m$ ) is significantly lower than the range of its nominal value; in other words, a difference between them of $0.36 \%$ is quite a bit larger than "the standard deviation of $m^{\prime \prime}$ of $0.10 \%$ given by $1 \sigma_{\mathrm{H}} /$ (c.n. $)^{0.5}$ where c.n. is the cathode number used in constructing the histogram. This fact, however, is very similar to results obtained in other facilities [32,33], suggesting to us to reconsider use of the nominal value for the purpose of comparisons with measured data. On the other hand, the arithmetic mean for C5 is consistent with its nominal value; the difference between them is within the standard deviation of $m$ of $0.03 \%$. The $\pm 1 \sigma_{\mathrm{H}}(0.18 \%)$ of the histogram for $\mathrm{C} 5$ is equivalent to \pm 100 years in its ${ }^{14} \mathrm{C}$ age of 11,750 years, which can be a measure of the confidence interval for one-year. This performance is comparable with the other facilities, e.g., a well-known carbon dating lab in Japan, the Paleo Labo Co.,Ltd [33,34]. For C1, although the arithmetic mean is $0.15 \mathrm{pMC}$, sometimes values are below $0.1 \mathrm{pMC}$. This scatter implies possibility of contamination during the sample preparation, which is supported by another fact described as follows. In contrast with $\mathrm{C} 1$ in the estimation of background, a high-purity synthetic graphite powder (No.072-03845 Wako Pure Chemical Industries, Ltd, Osaka, Japan) is used as a machine background samples. This powder is made from coke $\left({ }^{14} \mathrm{C}\right.$-free), and is directly poured into the cathode hole without any chemical preparations; thus, ${ }^{14} \mathrm{C}$ counts detected with the powder are due to machine background. We have continuously observed around $0.06 \mathrm{pMC}$ for the graphite powder, which shows potential for improvement for lowering the background due to chemical process.

\subsection{Inter-laboratory comparison testing}

Comparison of the results obtained in different laboratories on the same samples is fundamental to objectively assessing accuracy and system performance. Comparison tests were carried out twice, in 2010 and 2012, with another AMS facility, the JAEA-AMS-MUTSU, of the Aomori Research and Development Center, JAEA [35,36]. This facility has provided highquality ${ }^{129} \mathrm{I}$-and ${ }^{14} \mathrm{C}$-AMSs for environmental science studies, especially for marine transport properties of radio-isotopes, as well as for radiocarbon dating. The typical properties of the AMS system are as follows: a 3 MV Tandetron Cockcroft-Walton accelerator manufactured by High Voltage Engineering Europa, and the simultaneous injection system with the separator-combiner.

In the comparison test performed in 2012, the samples of the HOxII, C5, and C1 were prepared in the MUTSU, distributed to the TONO, and measured in both facilities. The measurement condition such as the duration time or the beam current was taken as the normal condition in each facility. Figure 7 shows results obtained in 2012. For the data analysis, the algorithm used in the TONO was employed. It can be seen for the C5, that there was no significant difference between data obtained in both facilities. The results of the $\mathrm{C} 1$ analyzed in the TONO are much lower than that for the MUTSU. This is mostly due to the fact that during ${ }^{14} \mathrm{C}$ counting, ${ }^{13} \mathrm{C}$ ions for the simultaneous injection also entered the accelerator continuously, thus the counting rate (or its possibility) of ${ }^{13} \mathrm{C}$ coming into the ionization detector is much higher for the simultaneous injection than for the sequential injection, in spite of filtering by the combination of magnetic 
and electrostatic analyzers. Consequently, the measurement of quite low concentration samples is suitable relative to use by the TONO. The detailed results and discussion for the series of comparison tests will be summarised in a JAEA report in the future.
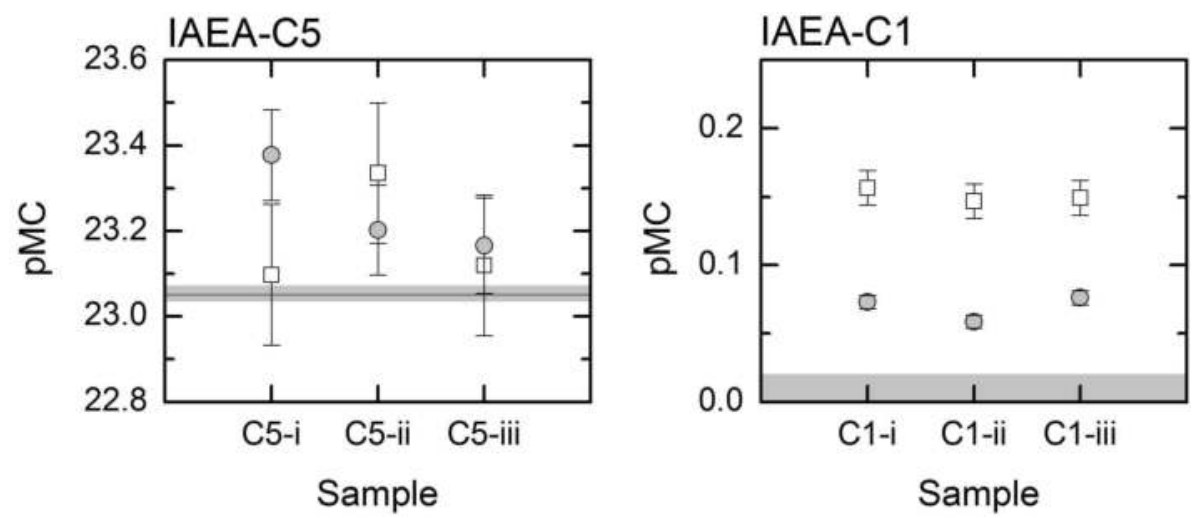

Figure 7. Results of comparison test between JAEA-AMS-TONO (shaded circles) and JAEA-AMS-MUTSU (empty squares). This test was carried out in 2012. The samples were prepared in the MUTSU, distributed to the TONO, and measured in both facilities. The algorithm for the data analysis followed the software code used in the TONO.

\section{5. ${ }^{10}$ Be measurement}

\subsection{System configuration and method}

The configuration for our ${ }^{10} \mathrm{Be}-\mathrm{AMS}$ operation is fundamentally standard, and is based on that used in the MALT $[18,37,38]$. Samples are made from the solid oxide of beryllium, $\mathrm{BeO}$, for its positive electron affinity to produce negative ions. Since the amount of the rare isotope ${ }^{10} \mathrm{Be}$ is distributed according to the abundance of oxygen isotope ratios, i.e., ${ }^{16} \mathrm{O}$ : ${ }^{17} \mathrm{O}:{ }^{18} \mathrm{O}=99.76: 0.04: 0.20$, respectively, the ${ }^{10} \mathrm{Be}^{16} \mathrm{O}$ is selected for injection into the accelerator to ensure high extraction efficiency of ${ }^{10} \mathrm{Be}$ from the sample. The terminal voltage, usually set at 4.7 or $4.8 \mathrm{MV}$, is made preferably as high as possible within the range of around 8 MV so as to increase the stripping efficiency from negative ions to 3+ (in our AMS system, the terminal voltage is limited to the specification of $5 \mathrm{MV}$ ). In addition, the higher ion energy is also preferred for ensuring the good performance of the discrimination between ${ }^{10} \mathrm{Be}$ and ${ }^{10} \mathrm{~B}$ for the heavy ion detector as described below (details in Section 5.2). One of the most significant features of ${ }^{10} \mathrm{Be}$ measurement is the simultaneous injection of ${ }^{9} \mathrm{Be}^{17} \mathrm{O}$ with mass the same as ${ }^{10} \mathrm{Be}^{16} \mathrm{O}$ for counting the abundance of ${ }^{9} \mathrm{Be}$ isotopes. For this purpose the current of ${ }^{17} \mathrm{O}^{5+}$ is measured to avoid uncertainty in the amount of beryllium hydroxide ${ }^{9} \mathrm{Be}^{16} \mathrm{OH}$ contamination in the sample [37]. The abundance isotope is detected with a Faraday cup behind the analyzing magnet as shown in Figure 5.

The mathematical formula used for obtaining the measured isotope ratio $\left(R_{\mathrm{m}}={ }^{10} \mathrm{Be} /{ }^{9} \mathrm{Be}\right)$ is: 


$$
R_{m}=\frac{\mathrm{a}_{10} B e}{\mathrm{a}_{9} B e} \frac{T_{17} O^{5+}}{T_{10} B e^{3+}} \frac{c p s_{10} B e^{3+}}{c p S_{17} O^{5+}},
$$

where $a$ is the correction factor for the abundance ratio of the oxygen isotopes, $T$ is transmittance for yielding positive ions from the negative ions, and cps is counts per second. The transmittance values for ${ }^{17} \mathrm{O}^{5+}$ and ${ }^{10} \mathrm{Be}^{3+}$ are substituted by the values for ${ }^{16} \mathrm{O}^{5+}(=21 \%)$ and ${ }^{10} \mathrm{Be}^{3+}(=6 \%)$, respectively. The absolute isotope ratio, $R_{\mathrm{A}}$, is obtained by correcting the measured ratio, as $R_{\mathrm{A}}=R_{\mathrm{m}} /$ Const, where Const is the normalization constant that is given by the measured ratio of a standard divided by its nominal ratio. For the ${ }^{10} \mathrm{Be}$ standard, usually so-called "ICN standard 01-5-1" (hereinafter simply S5-1) is used. The series of ICN standards has been prepared and distributed by Nishiizumi of $U$ of $C$, Berkeley to worldwide AMS laboratories [39].

The boron ions ${ }^{10} \mathrm{~B}^{3+}$ has the same charge-to-mass ratios as the ${ }^{10} \mathrm{Be}^{3+}$, thus it remains on the beamline regardless of the magnetic and electrostatic filters, and results in the entry into the ionization chamber, with respect to the isobar problem as mentioned in Section 3. Usually a gas or solid absorber technique has been used to discriminate between them. Therefore, optimization of the absorber is fundamental in the ${ }^{10} \mathrm{Be}$ measurement.

\subsection{Optimization of the rare isotope detector}

For the development of the ${ }^{10} \mathrm{Be}-\mathrm{AMS}$, discrimination between the ${ }^{10} \mathrm{Be}$ and ${ }^{10} \mathrm{~B}$ isotopes was accomplished by optimization of gas pressures in the ionization chamber and the absorber gas cell (hereinafter simply the gas-cell) attached in front of gas ionization chamber. Figure 8 shows the configuration of the rare isotope detector for the ${ }^{10} \mathrm{Be}-\mathrm{AMS}$ in our system. Ionization chamber consists of the cathode electrode (plate), grid, and anodes that are multiple (five) $\Delta E$ electrodes arranged along the axis of the beamline. The grid so called Frisch grid is to remove the dependence of the pulse amplitude on position of ion pair generation by ionization due to an incident particles.

As mentioned in Section 3.2, the $\Delta E$ electrode provides the pulse signal equivalent to the energy loss arising in the area corresponding to the electrode. For incident ion energy over a million electron volts, the rate of energy loss depends largely on the atomic number. Thus, the spectrum peak on the plane given by, e.g., both the coordinates $\Delta E_{1}$ and $E_{\text {Res }}$ (where "Res" means residual, see Figure 8) takes a characteristic position on that plane. This individuality of the peak position helps to discriminate the ${ }^{10} \mathrm{~B}$. However, the amount of ${ }^{10} \mathrm{~B}$ entering into the ionization chamber is enormous (over $10^{6}$ times larger than that of the ${ }^{10} \mathrm{Be}[40]$ ), so that the ${ }^{10} \mathrm{Be}$ signal is disturbed if there is no additional absorber for it. The gas-cell method is an absorbance technique for ${ }^{10} \mathrm{~B}$ based on the fact that the energy loss rate for the boron is expected to be larger than that for beryllium for the dependence of energy-loss rate on the atomic number. In our system, the gas-cell is prepared with 1-inch diameter pipe covered by the Havar and Mylar foils at both ends. The nitrogen gas pressure in the absorber significantly affects the shape of the ${ }^{10}$ Be spectrum, as described below. 


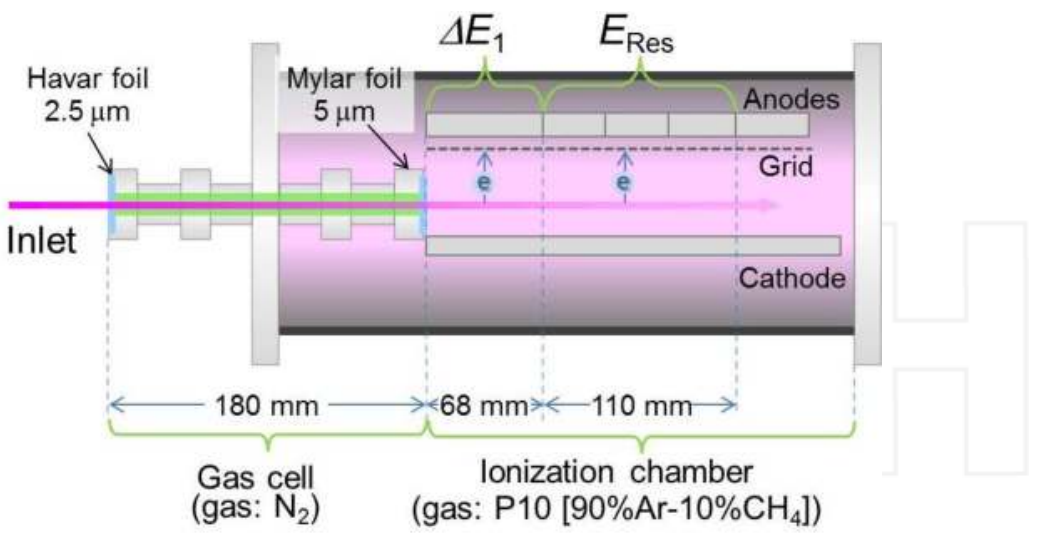

Figure 8. Configuration of the rare isotope detector (heavy ion detector) for ${ }^{10} \mathrm{Be}-\mathrm{AMS}$. The absorber gas-cell is attached in front of ionization chamber. The anode electrode is separated into multiple (five) $\Delta E$ electrodes. $\Delta E_{1}$ and $E_{\text {Res }}$ (that means Residual energy) indicate the energy loss arising in the region corresponding to the anode plate 1 and plates 2-4, respectively.

We investigated experimentally the discrimination function of the detector system through observation of the variation of the $\Delta E_{1}-E_{\text {Res }}$ spectrum by varying the gas pressure of the gas cell. Figure 9 shows the effect of gas pressure on the $\Delta E_{1}-E_{\text {Res }}$ spectrum. It can be seen that the horizontal width ( $\Delta E_{1}$ component) is reduced significantly with increasing $P_{\mathrm{C}}$. It should be noted that the gas pressure of the ionization chamber $P_{\mathrm{I}}$ is decreased with increasing $P_{\mathrm{C}}$, so as to balance between pulse heights for $\Delta E_{1}$ and $E_{\text {Res }}$, because the peak position on the Bragg curve (that provides the distribution of energy-loss rate as a function of the distance along the beam axis) shifts toward the gas-cell, as $P_{\mathrm{c}}$ is increased. There is a lower limit of $0.2 \mathrm{~V}$ in $E_{\mathrm{Res}}$ for data points. This is because the both pulse heights corresponding to $\left(\Delta E_{1}, E_{\mathrm{Res}}\right)$ coordinates of data points is acquired if the electrical voltage of the $E_{\mathrm{Res}}$-trace exceeds $0.2 \mathrm{~V}$ to discriminate between signal and noise. The middle and bottom frames in Figure 9 show the peak profiles in the $\Delta E_{1}$ component for ${ }^{10} \mathrm{Be}$ and ${ }^{10} \mathrm{~B}$, respectively. These peak profiles are well fitted with the Gaussian curve characterized by the standard deviation of $\sigma_{10 \mathrm{Be}}$ and $\sigma_{10 \mathrm{~B}}$.

The width of the ${ }^{10}$ Be peak defined by $\sigma_{10 \mathrm{Be}}$ is approximately $1 / 6$ smaller at $P_{\mathrm{C}}=58$ Torr than that at 32 Torr. As a measure of the discrimination function, the dimensionless normalized distance between ${ }^{10} \mathrm{Be}$ and ${ }^{10} \mathrm{~B}$ peaks given by

$$
\frac{\Delta}{\sqrt{\sigma_{10 B e, H}^{2}+\sigma_{10 B e, V}^{2}+\sigma_{10 B, H}^{2}+\sigma_{10 B, V}^{2}}}
$$


where, $\Delta$ is the distance between ${ }^{10} \mathrm{Be}$ and ${ }^{10} \mathrm{~B}$ peaks, and subscripts $\mathrm{H}$ and $\mathrm{V}$ mean horizontal and vertical, respectively. Figure 10 shows the experimentally obtained normalized distance with equation (2) as a function of $P_{\mathrm{C}}$. In the calculation of Equation (2) the value of $\sigma_{10 \mathrm{Be}, \mathrm{H}}$ is substituted in $\sigma_{10 \mathrm{~B}, \mathrm{H}}$ for the following two reasons. (i) The precision of $\sigma_{10 \mathrm{~B}, \mathrm{H}}$ is inadequate for higher $P_{C}$, because the peak of boron becomes so small that the main portion of the peak hides under the lower limit of $E_{\text {Res }}(0.2 \mathrm{~V})$ as shown in Figure 9. (ii) It has been experimentally observed that the relation $\sigma_{10 \mathrm{~B}, \mathrm{H}} \approx \sigma_{10 \mathrm{~B}, \mathrm{H}}$ holds independent of $P_{\mathrm{C}}$ within the range where $\sigma_{10 \mathrm{Be}, \mathrm{H}}$ and $\sigma_{10 \mathrm{~B}, \mathrm{H}}$ vary significantly in the same way with $P_{\mathrm{C}}$. It is found that the values of normalized distance peak at $P_{\mathrm{C}}=57$ Torr. This is due to the fact that as $P_{\mathrm{C}}$ increases over $55 \mathrm{Torr}$, the reduction of peak widths becomes saturated, while the distance $\Delta$ going into decline because of the enhancement in the energy loss in the gas-cell. It can be said that the optimum $P_{\mathrm{C}}$ is around 57 Torr providing the best discrimination. The nature of the $P_{\mathrm{C}}$-dependence on the peak-width is attributed to the baseline fluctuation of the pulse trace detected in the ionization chamber, which is briefly discussed in Section 7.
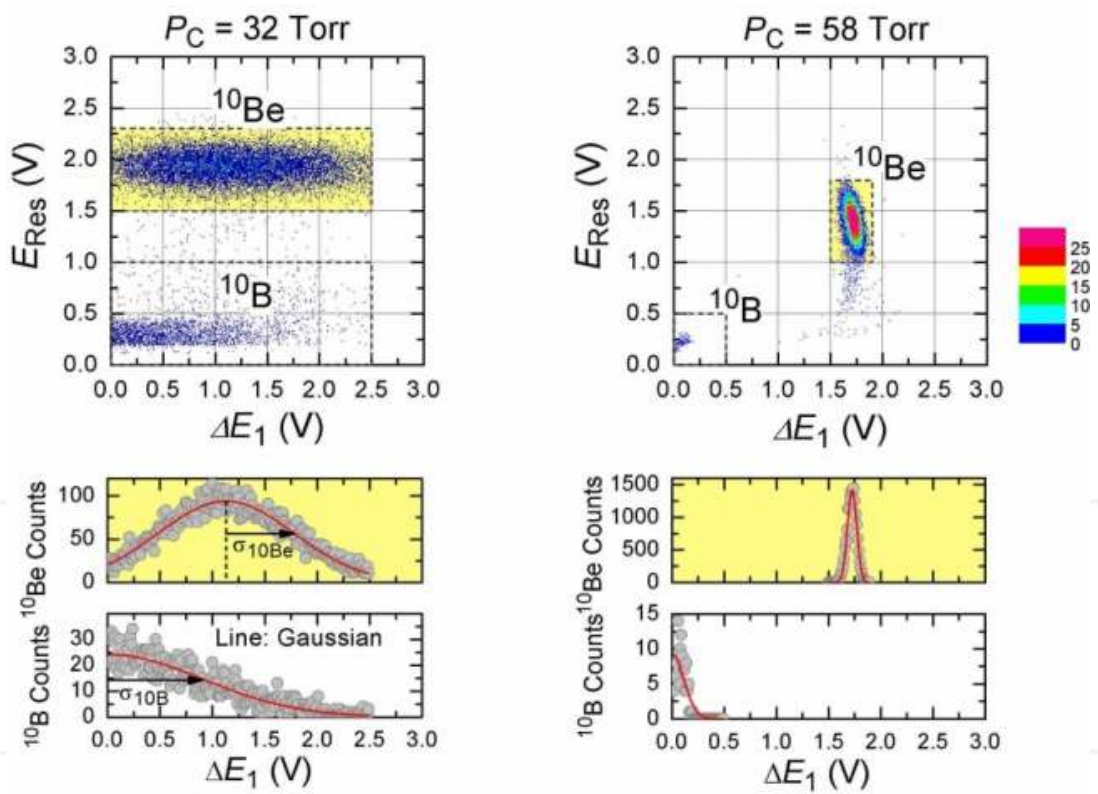

Figure 9. The variation of $\Delta E_{1}-E_{\text {Res }}$ spectrum for two gas pressures of the gas-cell, $P_{C}$ (upper frames). Corresponding peak profiles of ${ }^{10} \mathrm{Be}$ and ${ }^{10} \mathrm{~B}$ are also depicted for the $\Delta E_{1}$ component in the middle and bottom frames, respectively. The peak is fitted by a Gaussian distribution characterized with a standard deviation of $\sigma$. The gas pressure for the ionization chamber for left and right cases are 114 Torr and 56 Torr, respectively. 


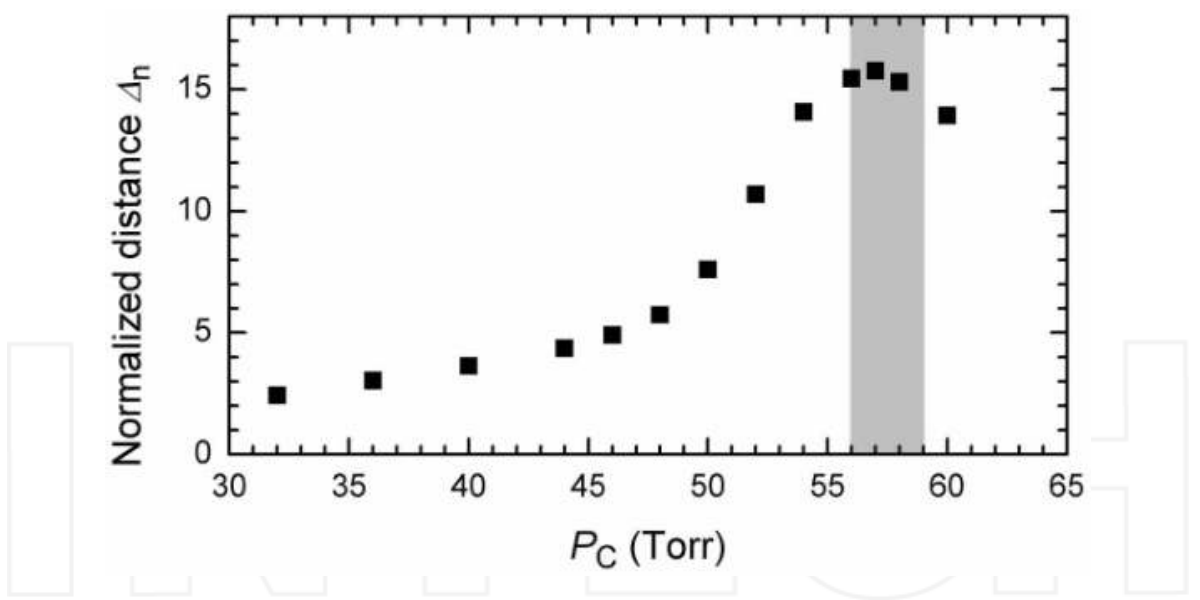

Figure 10. Variation of the normalized distance between ${ }^{10} \mathrm{Be}$ and ${ }^{10} \mathrm{~B}$ spectrum peaks as a function of the $P_{\mathrm{C}}$. The range of $P_{C}$ indicated by the shaded region is an optimized range, and is usually employed.

\subsection{Test measurements, Long-term reliability}

We completed the development of the ${ }^{10} \mathrm{Be}$ measurement technique last fiscal year (2012), confirming high stability and reliability of the ${ }^{10} \mathrm{Be} / \mathrm{Be}$ ratios in numerous test measurements. Even after we started to perform requested ${ }^{10} \mathrm{Be}$ measurements in 2013 , data quality has been continuously checked for every routine measurement using standards. Three typical standards are the ICN standards mention in the Section 5.1, S5-1, S5-2, S6-2, and a blank sample. The blank sample (hereafter BLK) is made from a quantified standard for atomic absorption spectrometry (No.020-07481) produced by Wako Pure Chemical Industries for which a ${ }^{10} \mathrm{Be} / \mathrm{Be}$ ratio of $\sim 2 \times 10^{-14}$ is expected [41]. As shown in Section 5.1, S5-1 is used to obtain the normalization constant given by the measured ratio of S5-1 divided by its nominal ratio. With respect to the data quality of the S5-1 standard, the relative precision, in terms of the relative standard deviation $r s d$ is important, since only the $r s d$ affects the normalization constant, where the role of the S5-1 standard is similar to that of HOxII standard in the ${ }^{14} \mathrm{C}$ measurement, as described in Section 4.1. The value of $r s d$ is around $0.50 \%$ for each routine measurement.

Figure 11 shows the quality of measurements from October 2011 to December 2013. The left column shows the evolution of the ${ }^{10} \mathrm{Be} / \mathrm{Be}$ ratios using the S5-2, S6-2, and BLK standards. The ${ }^{10} \mathrm{Be}$ measurements have been conducted at intervals ranging from around a few months to half a year. All ${ }^{10} \mathrm{Be} / \mathrm{Be}$ ratios for both S5-2 and S6-2 agree with the nominal values within $3 \sigma$ of each point, where the $\sigma$ is the combined uncertainty of the counting and the nominal ratio of the S5-1 standard. On the other hand, although the ratio of BLKs lies around its expected range, it seems to vary systematically up and down. This variation can be related to at least two factors: one is beam slit condition, and the other is the problem of the sample preparation. As shown in Figure 11, the time when the BLK ratio is low, in December 2012, coincides with the time when we began to narrow the beam slit behind the ECA to improve the ECA resolu- 
tion. The rise in the ratio after December 2012 implies that there could still remain a somewhat unidentified route for contamination in the sample preparation. This interpretation is supported by the observation that the ratio for the commercial, high-purity chemical reagent $\mathrm{BeO}$ powder produced by the Mitsuwa Chemical Co., Ltd. stabilized below 10-14 after Dec. 2012.
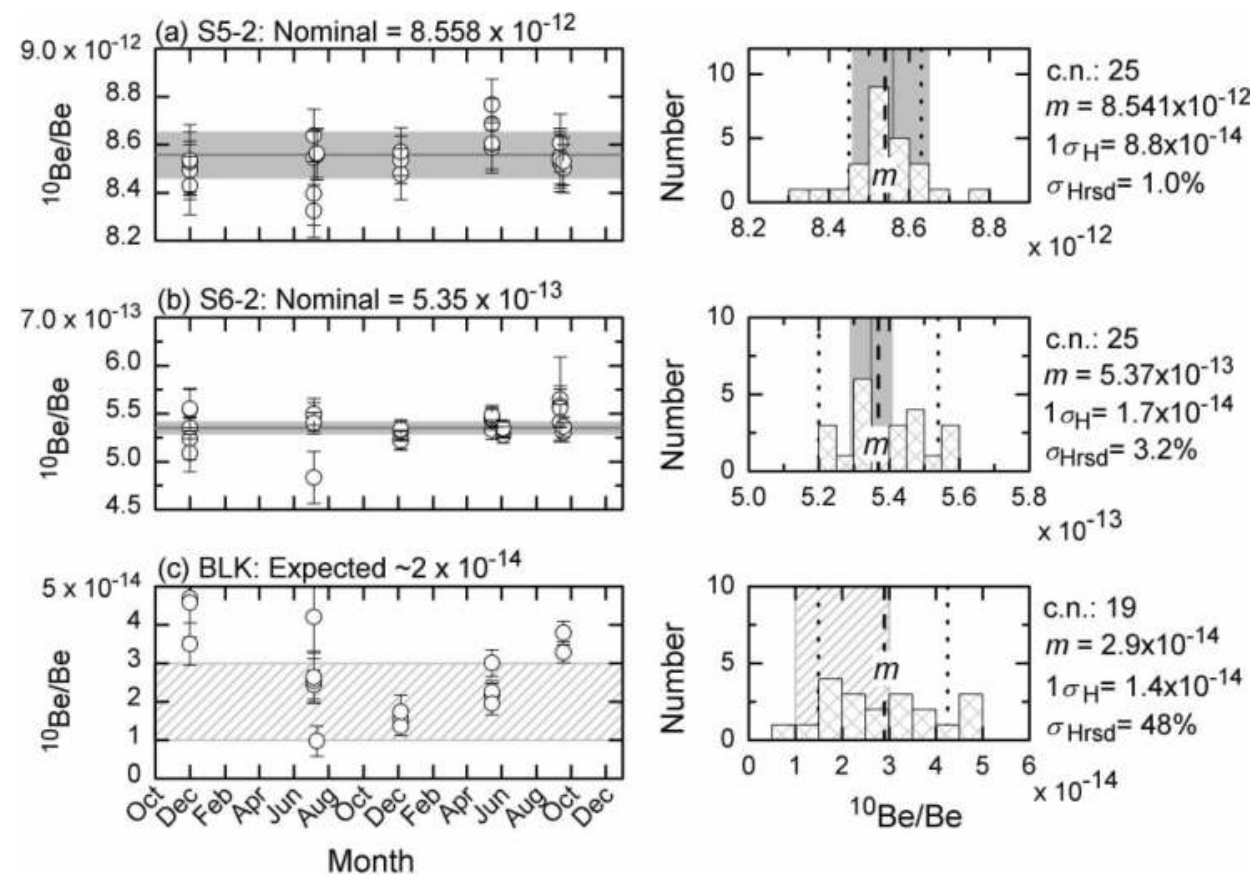

Figure 11. Measurement quality from October 2011 to December 2013 using ${ }^{10}$ Be-AMS standards: S5-2 (a), S6-2 (b), and blank BLK (c). The left column shows evolution of the measurement results. The gray line and hatching in both the left and right columns depicts respectively the nominal values and their uncertainty. The uncertainty range is based on a nominal uncertainty of $1.1 \%(1 \sigma)$ [39]. The right column depicts the corresponding histograms based on the left column measurement points. The dashed and dotted lines represent respectively the mean values $(m)$ and their uncertainty $\left(1 \sigma_{\mathrm{H}}\right)$. The symbol c.n. stands for the number of sampling cathodes used in building the histograms, and $\sigma_{\mathrm{Hrsd}}$ is the relative standard deviation of the $\sigma_{\mathrm{H}}$.

The frames on the right-hand side of Figure 11 are histograms corresponding to the left-hand frames. It can be seen that the value of $m$ (the arithmetic mean of the histogram) for the S5-2 and S6-2 standards are fairly consistent with corresponding nominal values; each difference between measured and nominal value is within the standard deviation of $m$, given by $1 \sigma_{\mathrm{H}} /$ (c.n.) $)^{0.5}$, i.e., $1.8 \times 10^{-14}$ for S5-2, and $0.34 \times 10^{-14}$ for S6-2.

\subsection{Comparison test}

We performed a comparison test with the AMS system in the MALT using beryllium samples made from an ice core. The samples measured in our system were originally prepared as spares 
for the ${ }^{10} \mathrm{Be}$ measurements that had already been performed in the MALT accelerator in 2010. In this test, therefore, the samples measured at both facilities are produced by the same process for the comparison.

Figure 12 and Table 2 show the results of the comparison test. Almost all of the measured ${ }^{10} \mathrm{Be} /$ Be ratios are consistent with the values obtained by the MALT AMS system. There is a significant difference between samples $\mathrm{B}$ and $\mathrm{H}$ (indicated by the arrow) taking into consideration their uncertainties, which could be due to unknown systematic errors. For the results of the ${ }^{10} \mathrm{Be} / \mathrm{Be}$ ratio measured in the MALT AMS system, two data sets are depicted for different data processing methods: one method is used at Hirosaki Univ. Japan, and the other is our method. The algorithms used for drawing the ${ }^{10} \mathrm{Be} / \mathrm{Be}$ ratios for both methods are almost the same $^{1}$, but the uncertainty has different interpretations, depending on which method is chosen. The uncertainty in the Hirosaki Univ., unc1, represents the standard deviation obtained by repeated measurements, whereas in our method, the uncertainty, unc2, is drawn basically by combining both the counting uncertainty of ${ }^{10} \mathrm{Be}$ and the uncertainty in the nominal value of S5-1 (1.1\% for $1 \sigma[39])$. The uncertainty in the nominal value affects that in the normalized constant, but is generally not always considered. This is because a lot number of standards does not change until the sample bottle is renewed, and thus the true value of the normalization constant rarely shifts. However, even when one assesses the data quality over a long time frame or performs an inter-laboratory comparison test, one should consider the difference in the lot number in order to accurately compare the isotope ratio values. Despite this fact, as shown in Table 2, there seems to be no systematic difference between unc1 and unc2, as expected. It is noted that the counting uncertainty itself is around $70 \%$ of unc1.

\begin{tabular}{c|rr|rrrr}
\hline \multirow{2}{*}{ Sample } & \multicolumn{2}{|c|}{ TONO } & \multicolumn{4}{c}{ MALT $^{\star}$} \\
\cline { 2 - 7 } & $\begin{array}{r}\text { Ratio } \\
\times 10^{-12}\end{array}$ & $\begin{array}{c}\text { unc } \\
\times 10^{-14}\end{array}$ & $\begin{array}{r}\text { Ratio1 } \\
\times 10^{-12}\end{array}$ & $\begin{array}{r}\text { unc1 } \\
\times 10^{-14}\end{array}$ & $\begin{array}{r}\text { Ratio2 } \\
\times 10^{-12}\end{array}$ & $\begin{array}{c}\text { unc2 } \\
\times 10^{-14}\end{array}$ \\
\hline A & 3.278 & 4.4 & 3.273 & 6.4 & 3.253 & 6.1 \\
\hline B & 6.252 & 8.0 & 6.637 & 14.4 & 6.617 & 9.9 \\
\hline C & 4.067 & 5.8 & 4.095 & 7.1 & 4.077 & 7.4 \\
\hline D & 4.406 & 5.6 & 4.379 & 9.3 & 4.362 & 7.2 \\
\hline E & 2.869 & 4.1 & 2.970 & 9.2 & 2.951 & 5.6 \\
\hline F & 3.846 & 5.0 & 3.967 & 4.8 & 3.945 & 6.1 \\
\hline G & 4.501 & 6.0 & 4.585 & 10.0 & 4.561 & 7.0 \\
\hline H & 4.414 & 5.6 & 4.643 & 5.6 & 4.620 & 7.1 \\
\hline I & 4.148 & 5.5 & 4.266 & 4.7 & 4.244 & 6.8 \\
\hline J & 3.618 & 4.8 & 3.688 & 5.7 & 3.666 & 6.2 \\
\hline
\end{tabular}

*Ratio1:HIROSAKI Univ., Ratio2: TONO.

Table 2. Results of the comparison test corresponding to the points in Figure 12.

1 In the calculation in the normalized constant, S5-1 and/or S5-2 were used atHirosaki Univ., but only S5-1 was used in the TONO. 


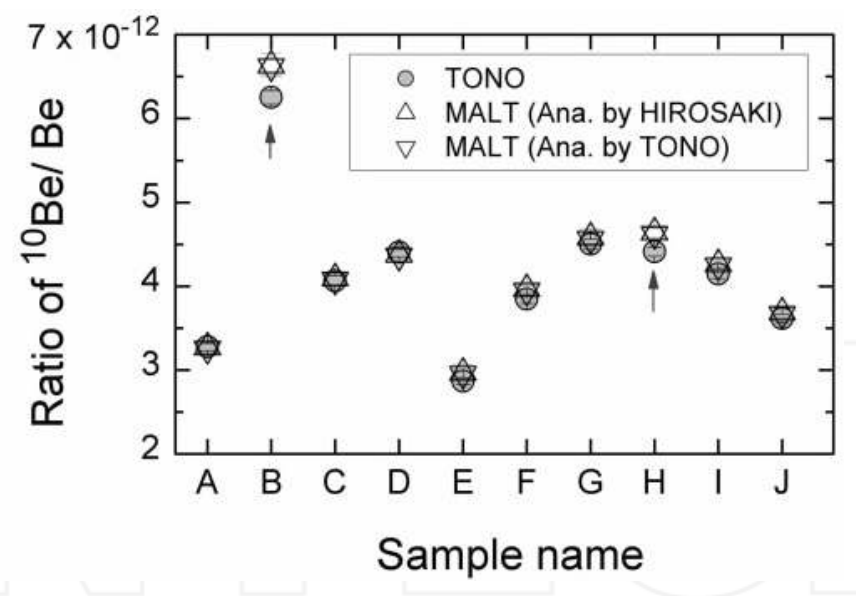

Figure 12. Comparison test using unknown samples.

On the other hand, the average uncertainty for the TONO (unc) is approximately $78 \%$ of that for the MALT (unc2). This is due to the fact that the total counts of the ${ }^{10} \mathrm{Be}$ signal for the TONO were three times larger than that for the MALT because of longer measurement time at the TONO. It should be mentioned that the average count rate is $70 \mathrm{cps}$ at the TONO but is 250 cps at the MALT AMS system, a reflection of the different specifications of their respective ion sources. Actually, the ion current for the MALT AMS system can provide a few times larger current than for the TONO.

\section{6. ${ }^{26} \mathrm{Al}$ measurement}

\subsection{Development and test measurements}

Tuning up of the system and the test measurements for the routine ${ }^{26} \mathrm{Al}-\mathrm{AMS}$ operation started in March of 2013, after the development of routine ${ }^{10} \mathrm{Be}$ measurements was finished. We plan to complete the development of the routine ${ }^{26} \mathrm{Al}$-AMS operation in the middle of fiscal year 2014, confirming the long term stability and reliability of the operation through statistical analysis of accumulated data.

For the ${ }^{26} \mathrm{Al}$ measurements, $\mathrm{Al}_{2} \mathrm{O}_{3}$ powder is chosen as the sample material (mixed with silver powder), and fundamentally no isobar problems occur because ${ }^{26} \mathrm{Mg}$ does not form negative ions. Therefore, the development of the ${ }^{26} \mathrm{Al}$-AMS procedure is more straightforward than the ${ }^{10} \mathrm{Be}-\mathrm{AMS}$ procedure. The configuration for ${ }^{26} \mathrm{Al}$-AMS is listed in Table 1.

Figure 13 shows the observed ${ }^{26} \mathrm{Al}^{3+}$ peak in the $E_{\mathrm{Tot}}-\Delta E_{1}$ spectrum. As described above, there is no other peak. The data points outside the counting gate (the green square) can be attributed to some incident ions reaching the electrode without complete volumetric energy-loss due to large-angle scattering at the window of the ionization chamber. 

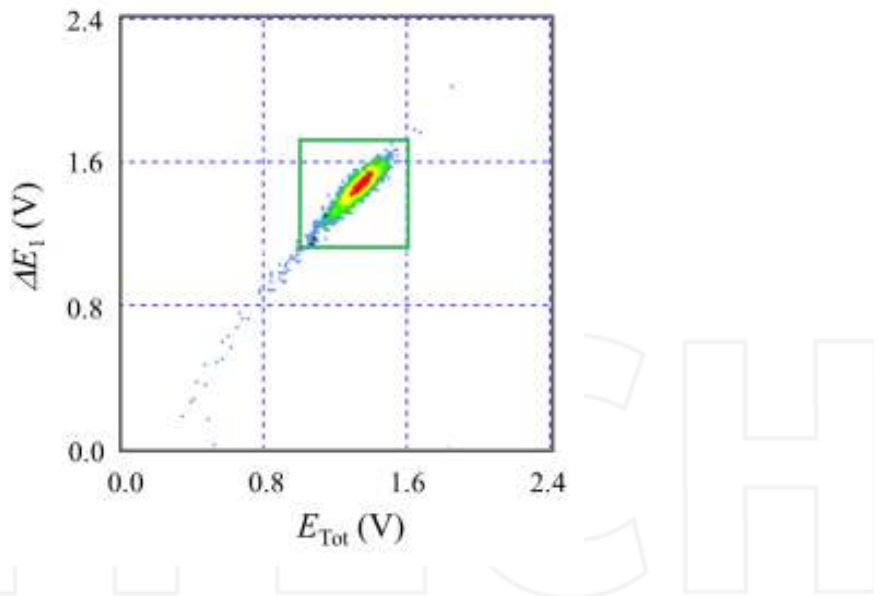

Figure 13. Example of the observed ${ }^{26} \mathrm{Al}{ }^{3+}$ peak in the $E_{\text {Tot }}-\Delta E_{1}$ spectrum.

We have performed test measurements using ${ }^{26} \mathrm{Al}$ standards, in order to investigate measurement stability. For the test measurements, the series of standards prepared and distributed by Nishiizumi have been used [42], as well as the ${ }^{10} \mathrm{Be}$ measurement mentioned in Section 5.3. Typical standards, 01-4-1 and 01-5-1 (S4-1 and S5-1, respectively), especially the former have been employed to compute the normalization constant that is given by the measured ratio of a standard S4-1 divided by its nominal ratio. The blank sample (BLK) was made from a quantified standard for atomic absorption spectrometry (No.016-15471) supplied by Wako Pure Chemical Industries.

The left column on Figure 14 shows the results of the two test measurements carried out between routine ${ }^{14} \mathrm{C}$ - and ${ }^{10} \mathrm{Be}-\mathrm{AMS}$ operations. Concerning the isotope ratio for the $\mathrm{S} 4-1$ standard, only the precision is meaningful (in other words, the accuracy has little meaning), since the arithmetic mean of the points obtained in the same batch (or on same date) is already normalized to the nominal value. A long-term precision can be drawn from the statistical dispersion of the data points displayed on the same figure, which will be mentioned in the below description related to the histogram. All data points for the S5-1 standard are consistent with the nominal value within $3 \sigma$, where $\sigma$ (indicated as error bars) is combined uncertainty from the statistical uncertainty and the uncertainty normalized constant (originated from the uncertainty of the nominal ratio of the S4-1 standard). On the other hand, the result of the BLK implies a decrease in the isotope ratio. This can be due to improvement of the energy resolution of the ECA accomplished by narrowing the beam slit located behind the ECA. It is believed that the contamination to the beamline originates in the ion source or in the high energy beamline where unwanted ions can exist because of the dissociation of molecular ions [5]. Although the expected isotope ratio for the BLK is not yet known, the observed range might be acceptable considering the range of the BLK ratio for ${ }^{10} \mathrm{Be}$ [see Section 5.3]. 

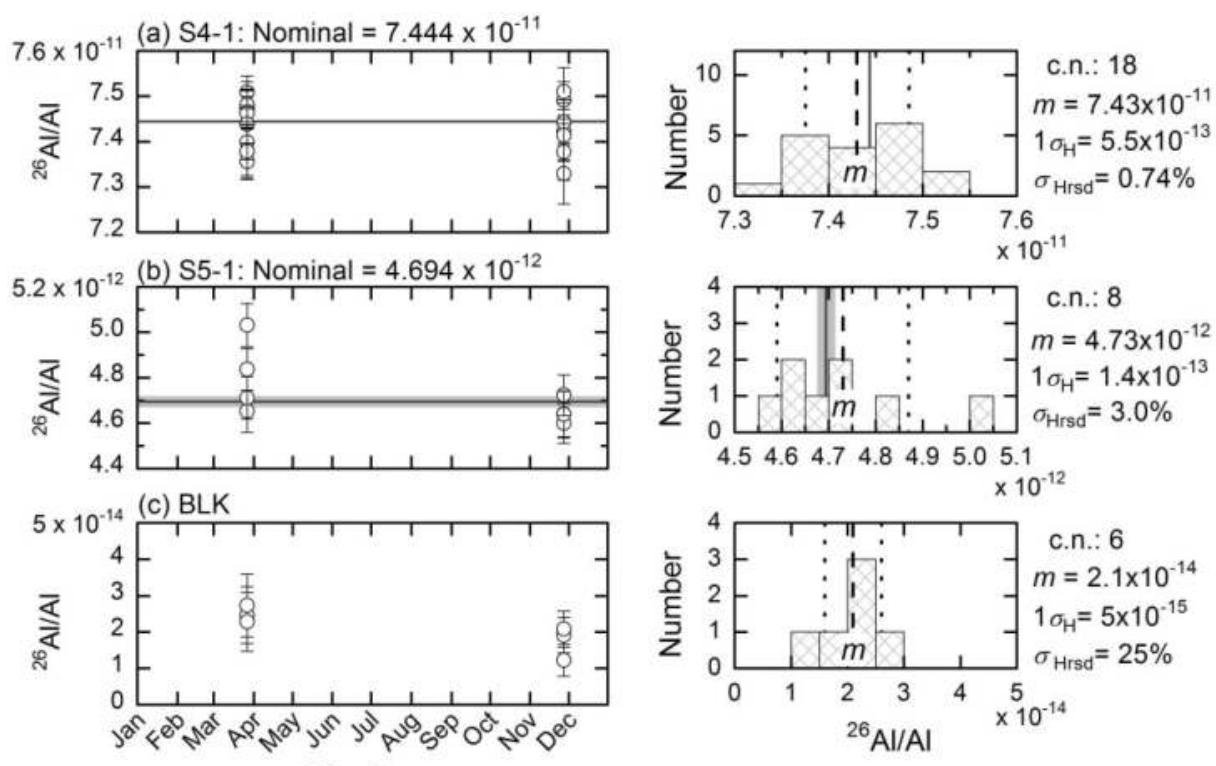

Month

Figure 14. Data quality in test measurements implemented from March 2013 for ${ }^{26} \mathrm{Al}$ standards: S4-1 (a), S5-1 (b), and BLK (c). The left column shows time series plots. The gray line and hatching in (b) for left and right frames indicate the nominal value and the range of its uncertainty, respectively. This range is based on the uncertainty of the absolute value of the nominal value $(0.37 \%$ for $1 \sigma[42])$. The right side shows corresponding histograms representing the left measurements. The dashed and dotted lines stand for arithmetical mean $(m)$ and uncertainty $\left(\sigma_{H}\right)$, respectively, for the histogram. The symbol c.n. denotes the number of cathodes used for building the histogram, and $\sigma_{\text {Hrsd }}$ is the relative standard deviation of $\sigma_{\mathrm{H}}$.

The histograms in the right-side frames in Figure 14 present the data distributions shown as data points in the left-side frames. The long-term precision of the S4-1 standard can be indicated by the statistical dispersion of their points in Figure 14a labelled as $\sigma_{\mathrm{H}}$. The relative uncertainty of $\sigma_{\mathrm{H},}$ or $\sigma_{\mathrm{Hrsd}}(=0.74 \%)$ is less than $0.9 \%$. This magnitude relation, or $\sigma_{\mathrm{Hrsd}}<0.9 \%$, provides a necessary condition that the precision of the S5-1 standard would be less than $4 \%$ where the value itself is comparable to the precision in the ${ }^{26} \mathrm{Al}$ measurements in the MALT AMS system [18]. Here we assume that the ${ }^{10} \mathrm{Be}$ counts are simply proportional to the nominal ratio, and the S5-1 standard is regarded as representative of ${ }^{26} \mathrm{Al}$ standards except the S4-1 standard. Actually, the nominal ${ }^{26} \mathrm{Al} / \mathrm{Al}$ ratio of the S5-1 standard is nearest to a log-average (or a geometric mean) for all the ${ }^{26} \mathrm{Al}$-AMS standards. As shown in the histogram of the S5-1 standard, the precision is, indeed, less than $4 \%$. Furthermore, the difference between $m$ and

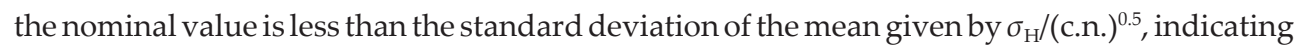
there is consistency between them. For all the results shown in this figure, data points are inadequate for a statistical discussion; thus we will need to acquire additional data for evaluation of the measurement stability, in order to develop ${ }^{26} \mathrm{Al}$ routine measurements. 


\section{Research and development: Baseline fluctuation of the ${ }^{10} \mathrm{Be}$ pulse trace}

As described in Section 5.2, we saw that discrimination between ${ }^{10} \mathrm{Be}$ and ${ }^{10} \mathrm{~B}$ in the ${ }^{10} \mathrm{Be}$-AMS is strongly dependent on the gas pressure of the gas-cell $\left(P_{\mathrm{C}}\right)$ located in front of gas ionization chamber. The $\Delta E_{1}$ component of the ${ }^{10} \mathrm{Be}$ peak on the $\Delta E_{1}-E_{\mathrm{Res}}$ spectrum shrinks significantly with decreased $P_{C}$ value. This shrinking, it should be emphasized, accompanies the reduction of the $\Delta E_{1}$ component of the ${ }^{10} \mathrm{~B}$ (see Figure 9), implying the shape of the ${ }^{10} \mathrm{Be}$ peak is closely related to the occurrence of incident ${ }^{10} \mathrm{~B}$, an interfering particle, is briefly discussed.

The peak width is a reflection of the statistical dispersion of the pulse height of signal traces detected from the ionization chamber. Figure 15 shows pulse traces observed by the $\Delta E_{1^{-}}$ electrode for the two $P_{\mathrm{C}}$ values corresponding to Figure 9 . One can see that the fluctuation of the baseline of the ${ }^{10} \mathrm{Be}$ signal is greater for the lower $P_{\mathrm{C}}$. It is noted that there was no remarkable fluctuation in the pulse trace observed by the $E_{\text {Res }}$-electrode; this indicates the dependence of the fluctuation on the distance from the inlet of ionization chamber. In addition, the fluctuation contains frequency components of around a few tenths of a kilohertz. It was observed that the frequency of the major component seems to decrease as $P_{\mathrm{C}}$ increases.

Figure 16 shows both the variation of the standard deviation of the fluctuation ( $\sigma$ in Figure 15 ) and the energy loss of ${ }^{10} \mathrm{~B}$ in the area of $\Delta E_{1}, \Delta E_{1}{ }^{10 \mathrm{~B}}$ (evaluated using SRIM [43]) as a function of $P_{\mathrm{C}}$. The values of $\sigma$ and $\Delta E_{1}{ }^{10 \mathrm{~B}}$ decrease in a similar way with increasing $P_{\mathrm{C}}$, indicating that the fluctuation can be closely related to the incident energy of the ${ }^{10} \mathrm{~B}$.
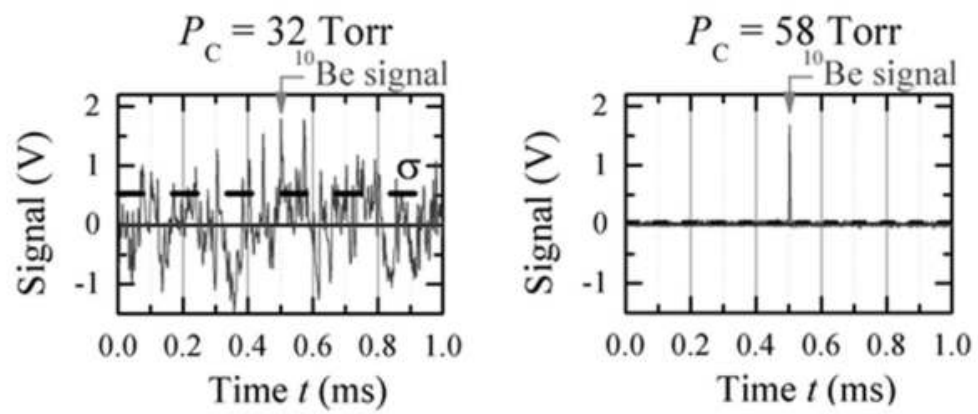

Figure 15. Pulse traces detected by the $\Delta E_{1}$-electrode in the ionization chamber for two values of $P_{\mathrm{C}}$ corresponding to those shown in Figure 9. The trigger for acquiring the pulse trace is taken from the pulse detected simultaneously by the $E_{\text {res }}$-electrode at $t=0.5 \mathrm{~ms}$. The dashed line shows the standard deviation of the fluctuation.

In general, if the incident frequency can no longer be ignored comparing the reciprocal of the time scale for the pulse width, a high counting rate induces pulse pile-up, and deteriorates the time resolution of the ionization chamber. Our investigation, by measuring the ${ }^{10} \mathrm{~B}$ current shows that the average frequency of the ${ }^{10} \mathrm{~B}$ incident is on the order of a megahertz, which is comparable to the reciprocal of the pulse width. In fact, the amount of ${ }^{10} \mathrm{~B}$ entering toward the ionization chamber is expected to be over $10^{6}$ times larger than that of ${ }^{10} \mathrm{Be}[40]$. The mechanism for the baseline fluctuation, however, is independent of the signal pile-up, but can be due to 


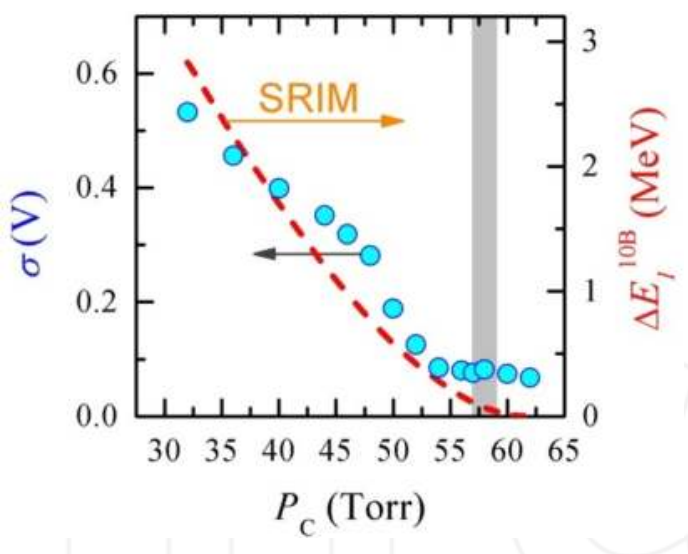

Figure 16. $\mathrm{P}_{\mathrm{C}}$-dependence on the standard deviation of the fluctuation $\sigma$. The energy loss of ${ }^{10} \mathrm{~B}$ in the $\Delta E_{1}$ region, $\Delta E_{1}{ }_{10 B}$, deduced by using the SRIM is also shown. The shaded hutch depicts the $P_{\mathrm{c}}$-range normally used.

the effect of the charge accumulation in the ionization chamber, as qualitatively described below.

In ordinary cases, electrons (negative charges) and ions (positive charges) produced by the ionization caused by the incident ion colliding with atoms in the ionization chamber drift toward the anode and cathode, respectively, in the applied electric field, and finally lose their charge at the electrodes. In the present case, it should be noted that the time interval of the ${ }^{10} \mathrm{~B}$ incident is much shorter than the time scale for the ions-loss on the order of milliseconds for the ordinary condition as mentioned above. This can lead to the charge accumulation in the space; the positive charge reaches a certain level so as to provide a balance between production rate and loss rate. The substantial positive charge lowers the anode potential through the inefficiency of the Frisch-grid playing a role in shielding the charges [44,45]. If some instability exists inherently in the relationship between the enhancement of the charge and the ion loss system, the anode potential, therefore, can fluctuate around its equilibrium value. Volumetric ion-electron recombination would be a candidate system for causing an instability so as to enhance the fluctuation of positive charge. This kind of degradation of the performance of the ionization chamber caused by the residual positive charge is not just related to the ${ }^{10} \mathrm{Be}$ measurement, but to more general measurements of rare isotopes accompanied by the isobar problem. Indeed, an effect of remaining charge was mentioned in a paper for improving the discrimination of ${ }^{36} \mathrm{~S}$ in ${ }^{36} \mathrm{Cl}$-AMS [46]. Therefore, it can be said that the investigation of the nature of pulse trace presented here has been conducted as a preparatory activity in the development of the ${ }^{36} \mathrm{Cl}$-AMS operation.

\section{Summary}

The AMS system operating at the Tono Geoscience Center (TGC) has not only continued to contribute reliable routine AMS measurements, but also made steady progress in developing 
multi-nuclide AMS in order to provide geochronological dating methods applicable to the entire Quaternary timescale.

Our versatile AMS system, based on the 5 MV Pelletron ${ }^{\mathrm{TM}}$ tandem accelerator, is designed for AMS analysis of most radio-isotopes including ${ }^{10} \mathrm{Be},{ }^{14} \mathrm{C},{ }^{26} \mathrm{Al},{ }^{36} \mathrm{Cl}$, and ${ }^{129} \mathrm{I}$. The AMS system is in good condition after fifteen years of operation, ensured by regularly scheduled maintenance. Total measurement time has been increasing for the last 15 years, and reached 15,000 hours this year. The average annual number of samples measured is 800 , and the grand total number of samples will exceed 12,500 within a few months.

In the ${ }^{14} \mathrm{C}$-AMS operation, the long-term reliability of routine measurements has been continuously verified by measuring standard samples such as $\mathrm{C} 1, \mathrm{C} 5$, and so on, produced by the IAEA, and HOxII produced by the NIST and by comparative testing with other AMS facilities. Almost all the relative standard deviations of the isotope ratios of HOxII in percent modern carbon are less than $0.25 \%$ for each measurement, and the average isotope ratio of $\mathrm{C} 1$ lies around $0.15 \mathrm{pMC}$. The comparison tests were carried out twice, in 2010 and in 2012, with the AMS facility at the JAEA-AMS-MUTSU. The results showed that there was no significant difference in the data obtained from the facilities.

With respect to the ${ }^{10} \mathrm{Be}-\mathrm{AMS}$ operation, we completed the development of ${ }^{10} \mathrm{Be}$ measurement capability last year, confirming both high stability and reliability of the ${ }^{10} \mathrm{Be} / \mathrm{Be}$ ratios obtained from numerous test measurements. Then, routine measurements started since the beginning of fiscal year 2013. The detection limit of the isotope ratio can be less than $7 \times 10^{-15}$, estimated by using samples made of commercial high-purity BeO powders. For the development of ${ }^{10} \mathrm{Be}-$ AMS, discrimination of ${ }^{10} \mathrm{Be}$ from ${ }^{10} \mathrm{~B}$ was accomplished by optimization of gas pressure in the gas cell located in front of gas ionization chamber. We also performed a comparison test with the AMS system at the MALT in the University of Tokyo using beryllium samples taken from an ice core. Measured ${ }^{10} \mathrm{Be} / \mathrm{Be}$ ratios were consistent with the values obtained by the MALT group, confirming the reliability of our measurements.

We have now entered into the development of ${ }^{26} \mathrm{Al}$-AMS. This development and the test measurement have progressed and have shown satisfactory results. We have performed system tuning and test measurements using ${ }^{26} \mathrm{Al}$ standard samples. Almost all measured ratios of ${ }^{26} \mathrm{Al} / \mathrm{Al}$ are consistent with nominal values, within the range of their uncertainty and routine measurements of ${ }^{26} \mathrm{Al}$ will start in the near future.

We have also conducted investigations for improving the heavy ion detection system based on the $\Delta E$ - $E_{\text {Res }}$ type-gas ionization chamber with multi-anodes. It has been observed that the high incident ion rate of the stable isobars into the gas ionization chamber disturbs the baseline of the pulse trace for the measured rare nuclide. This can be related to the fact that the remaining positive charge produced by isobars makes the anode signal fluctuate, which will be one of key factors that should be resolved for achieving the ${ }^{36} \mathrm{Cl}$-AMS with good discrimination of ${ }^{36} \mathrm{~S}$, a stable isobar. The development for the ${ }^{36} \mathrm{Cl}$-AMS analysis will be one focus for development work in the next few years. 


\section{Acknowledgements}

We would like to express our gratitude to Prof. Matsuzaki of the University of Tokyo for his continuous academic and practical advice. We would like to offer our special thanks also to Dr. Horiuchi of Hirosaki University for providing unknown beryllium samples and measurement data. Special thanks to Mr. Hanaki of the facility administrator for his management support and constant encouragement. We also thank the staff members of the AMS laboratory for their help and support.

\section{Author details}

Akihiro Matsubara $^{1 *}$, Yoko Saito-Kokubu ${ }^{1}$, Akimitsu Nishizawa ${ }^{2}$, Masayasu Miyake $^{2}$, Tsuneari Ishimaru ${ }^{1}$ and Koji Umeda ${ }^{1}$

*Address all correspondence to: matsubara.akihiro@jaea.go.jp

1 Tono Geoscience Center, Japan Atomic Energy Agency, Jorinji, Izumi, Toki, Japan

2 Pesco Corp., Ltd., Tokiguchiminami, Toki, Japan

\section{References}

[1] GIRDD. Geological Isolation Research and Development Directorate (GIRDD). Available: http://www.jaea.go.jp/04/tisou/english/index/e-index.html. Accessed 5 January 2014.

[2] Asamori K, Niwa M, Hanamuro T, Yamada K, Kusano T, et al. Annual Report for Research on Geosphere Stability for Long-Term Isolation of Radioactive Waste in Fiscal Years 2011. JAEA-Research 2012; 24.

[3] Yasue K, Asamori K, Niwa M, Hanamuro T, Saito-Kokubu Y, et al. Research Plan on Geosphere Stability for Long-Term Isolation of Radioactive Waste (Scientific Programme for Fiscal Year 2013). JAEA-Review 2013; 023.

[4] Finkel RC, Suter M. AMS in the Earth Sciences: Technique and Applications. Advances in Analytical Geochemistry; 1993: 1-114.

[5] Tuniz C, Bird JR, Fink D, Gregory F H. Accelerator Mass Spectrometry: Ultrasensitive Analysis for Global Science. CRC Press; 1998.

[6] Muzikar P, Elmore D, Granger DE. Accelerator mass spectrometry in geologic research. Geological Society of America Bulletin 2003; 115: 643-654. 
[7] IVY-OCHS S, KOBER F. Surface exposure dating with cosmogenic nuclides. Quaternary Science Journal (Eiszeitalter und Gegenwart) 2008; 57: 179-209.

[8] Asamori K, Niwa M, Hanamuro T, Yamada K, Kusano T, et al. Annual Report for Research on Geosphere Stability for Long-Term Isolation of Radioactive Waste in Fiscal Years 2012. JAEA-Research 2014; in press.

[9] Xu S, Ito S, Iwatsuki T, Abe M, Watanabe M. A new AMS facility at the JNC Tono Geoscience Center, Japan. Nuclear Instruments and Methods in Physics Research Section B: Beam Interactions with Materials and Atoms 2000; 172: 8-12.

[10] Itoh S, Abe M, Watanabe M, Nakai S, Touyama H, et al. Present status of the JNC Tono Geoscience Center AMS system. Nuclear Instruments and Methods in Physics Research Section B: Beam Interactions with Materials and Atoms 2004; 223-224: 100103.

[11] Saito-Kokubu Y, Nishizawa A, Suzuki M, Ohwaki Y, Nishio T, et al. Current status of the AMS facility at the Tono Geoscience Center of the Japan Atomic Energy Agency. Nuclear Instruments and Methods in Physics Research Section B: Beam Interactions with Materials and Atoms 2013; 294: 43-45.

[12] Matsubara A, Kokubu Y, Nishizawa A, Owaki Y, Nishio T, et al. Current status of JAEA-AMS-TONO, the Japan Atomic Energy Agency (2011). 4th East Asian Symposium on Accelerator Mass Spectrometry (EA-AMS-4). Tokyo. pp. 147-150.

[13] Matsubara A, Saito-Kokubu Y, Nishizawa A, Miyake M, Ishimaru T. System technology for ${ }^{10} \mathrm{Be}$ and ${ }^{26} \mathrm{Al}$ measurements in the JAEA-AMS-TONO (2013). 5th East Asia AMS Symposium (EA-AMS-5). Daejeon. p. 53.

[14] Saito-Kokubu Y, Ishimaru T, Matsubara A, Nishizawa A, Miyake M, et al. Present status of JAEA-AMS-TONO; Progress of multi nuclide AMS (2013). 5th East Asia AMS Symposium (EA-AMS-5). Daejeon. p. 22.

[15] Matsubara A, Nishizawa A, Miyake M, Saito-Kokubu Y, Ishimaru T. Development of System Techonology for Routine ${ }^{10} \mathrm{Be}$ Measurement in the JAEA-AMS-TONO (in Japanese) (2013). Proceedings of the 15th Japaneses Symposium on Accelerator Mass Spectrometry. Nagoya. pp. 25-28.

[16] JAEA. Procedure of shared use of JAEA facilities. Available: http://sangaku.jaea.go.jp/3-facility/05-support/jaea-facilities-eng.html. Accessed 8 January 2013.

[17] Sundquist M. 15SDH-2 AMS SYSTEM MANUAL. National Electrostatics Corp.; 1998.

[18] Matsuzaki H, Nakano C, Tsuchiya Y (Sunohara), Kato K, Maejima Y, et al. Multi-nuclide AMS performances at MALT. Nuclear Instruments and Methods in Physics Research Section B: Beam Interactions with Materials and Atoms 2007; 259: 36-40.

[19] Kume H, Shibata Y, Tanaka A, Yoneda M, Kumamoto Y, et al. The AMS facility at the National Institute for Environmental Studies (NIES), Japan. Nuclear Instruments 
and Methods in Physics Research Section B: Beam Interactions with Materials and Atoms 1997; 123: 31-33.

[20] Xu S, Anderson R, Bryant C, Cook G. Capabilities of the new SUERC 5MV AMS facility for 14C dating. Radiocarbon 2004; 46: 59-64.

[21] Maden C, Anastasi P.A.F., Dougans A, Freeman S.P.H.T., Kitchen R, et al. SUERC AMS ion detection. Nuclear Instruments and Methods in Physics Research Section B: Beam Interactions with Materials and Atoms 2007; 259: 131-139.

[22] Alfimov V, Possnert G, Aldahan a. Measurements of ${ }^{36} \mathrm{Cl}$ with a gas-filled magnet at the Uppsala tandem laboratory. Nuclear Instruments and Methods in Physics Research Section B: Beam Interactions with Materials and Atoms 2007; 259: 199-203.

[23] Salehpour M, Håkansson K, Possnert G. Accelerator mass spectrometry of ultrasmall samples with applications in the biosciences. Nuclear Instruments and Methods in Physics Research Section B: Beam Interactions with Materials and Atoms 2013; 294: 97-103.

[24] Stocker M, Döbeli M, Grajcar M, Suter M, Synal H-A, et al. A universal and competitive compact AMS facility. Nuclear Instruments and Methods in Physics Research Section B: Beam Interactions with Materials and Atoms 2005; 240: 483-489.

[25] Kerver S. Compact Universal AMS (UAMS) Systems. Available: http://www.pelletron.com/compactams.htm. Accessed 8 January 2014.

[26] Hong W, Hun J, Ki P, Sung S, Joo H, et al. A New 1MV AMS Facility at KIGAM. Raiocarbon 2010; 52: 243-251.

[27] Matsubara A, Nishizawa A, Suzuki M, Owaki Y, Nishio T, et al. Improvements of the JAEA-AMS-TONO; FY2009 (2010). 23th Proceedings of research workshop on tandem accelerator and its related technology (in Japanese). Tokyo. pp. 53-56.

[28] Southon JR, Santos GM. Ion Source Development at KCCAMS, University of California, Irvine. Radiocarbon 2004; 46: 33-39.

[29] Travertine M, Wood C, Sucrose W. Reference Sheet for Quality Control Materials. Available: http://nucleus.iaea.org/rpst/Documents/rs_IAEA-C1_to_IAEA-C9.pdf.

[30] "abc" NEC AMS Analysis Program, Version 6.1. National Electrostatics Corp.; 2005.

[31] Stenström KE, Skog G, Georgiadou E, Genberg J, Johansson A. A Guide to Radiocarbon Units and Calculations. LUNFD6 2011; 6: 1-17.

[32] Xu X, Khosh M, Druffel-Rodriguez K, Trumbore S, Southon J. Is the Consensus Value of ANU Sucrose (IAEA C-6) Too High? Radiocarbon 2010; 52: 866-874.

[33] Sato M, Itoh S, AHN S, Hirota M, Yamagata H, et al. Current status of the Compact AMS system at Paleo Labo Co., Ltd. (2013). Proceedings of the 15th Japaneses Symposium on Accelerator Mass Spectrometry. Nagoya. pp. 17-20. 
[34] Kobayashi K, Niu E, Itoh S, Yamagata H, Lomtatidze Z, et al. The compact ${ }^{14} \mathrm{C}$ AMS facility of Paleo Labo Co., Ltd., Japan. Nuclear Instruments and Methods in Physics Research B 2007; 259: 31-35.

[35] Aramaki T, Mizushima T, Mizutani Y, Yamamoto T, Togawa O, et al. The AMS facility at the Japan Atomic Energy Research Institute (JAERI). Nuclear Instruments and Methods in Physics Research Section B: Beam Interactions with Materials and Atoms 2000; 172: 18-23.

[36] Kabuto S, Kinoshita N, Tanaka T, Yamamoto N. Operational Status of the JAEAMUTSU Tandetron AMS 2008-2009. Prosceedings of the Second JAEA Tandetron AMS Utilization Workshop: November 12-13, 2009, Mutsu-shi, Japan. JAEA. pp. 126129.

[37] Imamura M, Hashimoto Y, Yoshida K, Yamane I, Yamashita H, et al. Tandem Accelerator Mass Spectrometery of ${ }^{10} \mathrm{Be} /{ }^{9} \mathrm{Be}$ with Internal Beam Monitor Method. Nuclear Instruments and Methods in Physics Research B 1984; 5: 211-216.

[38] Matsuzaki H, Tanikawa M, Kobayashi K, Hatori S. Development of a gas counter for AMS measurement of ${ }^{10} \mathrm{Be}$ and ${ }^{26} \mathrm{Al}$ of cosmic spherules. Nuclear Instruments and Methods in Physics Research B 2000; B 172: 218-223.

[39] Nishiizumi K, Imamura M, Caffee MW, Southon JR, Finkel RC, et al. Absolute calibration of ${ }^{10} \mathrm{Be}$ AMS standards. Nuclear Instruments and Methods in Physics Research Section B: Beam Interactions with Materials and Atoms 2007; 258: 403-413.

[40] Grajcar M. New concepts of ${ }^{10}$ Be Accelerator Mass Spectrometry at low energies. Swiss Federal Institute of Technology; 2005.

[41] Horiuchi K, Oniyanagi I, Wasada H, Matsuzaki H. ${ }^{10}$ Be measurements at MALT using reduced-size samples of bulk sediments. Nuclear Instruments and Methods in Physics Research Section B: Beam Interactions with Materials and Atoms 2013; 294: 72-76.

[42] Nishiizumi K. Preparation of ${ }^{26} \mathrm{Al}$ AMS standards. Nuclear Instruments and Methods in Physics Research Section B: Beam Interactions with Materials and Atoms 2004; 223-224: 388-392.

[43] Ziegler JF, Ziegler MD, Biersack JP. SRIM - The stopping and range of ions in matter (2010). Nuclear Instruments and Methods in Physics Research Section B: Beam Interactions with Materials and Atoms 2010; 268: 1818-1823.

[44] Bunemann O, Cranshaw TE, Harvey JA. Design of grid ionization chambers. Canadian Journal of Research 1949; 27: 191-206.

[45] Al-Adili A, Hambsch F-J, Bencardino R, Oberstedt S, Pomp S. Ambiguities in the grid-inefficiency correction for Frisch-Grid Ionization Chambers. Nuclear Instruments and Methods in Physics Research A 2012; 673: 116-121. 
[46] Martschini M, Andersson P, Forstner O, Golser R, Hanstorp D, et al. AMS of ${ }^{36} \mathrm{Cl}$ with the VERA 3MV tandem accelerator. Nuclear Instruments and Methods in Physics Research Section B: Beam Interactions with Materials and Atoms 2013; 294: 115120.
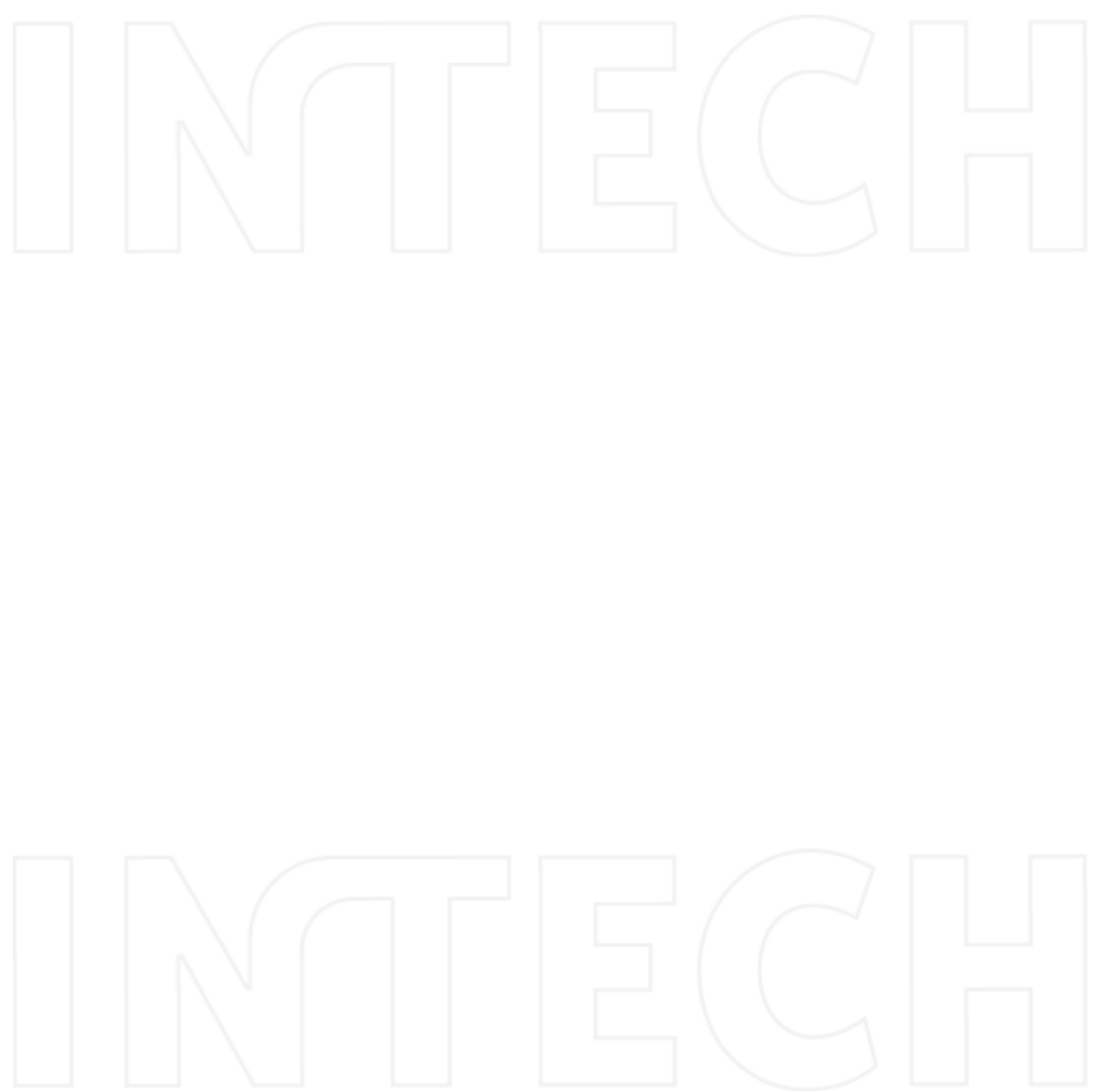


\title{
Chapter 2
}

\section{Luminescence Chronology}

\author{
Ken Munyikwa
}

Additional information is available at the end of the chapter

http://dx.doi.org/10.5772/58554
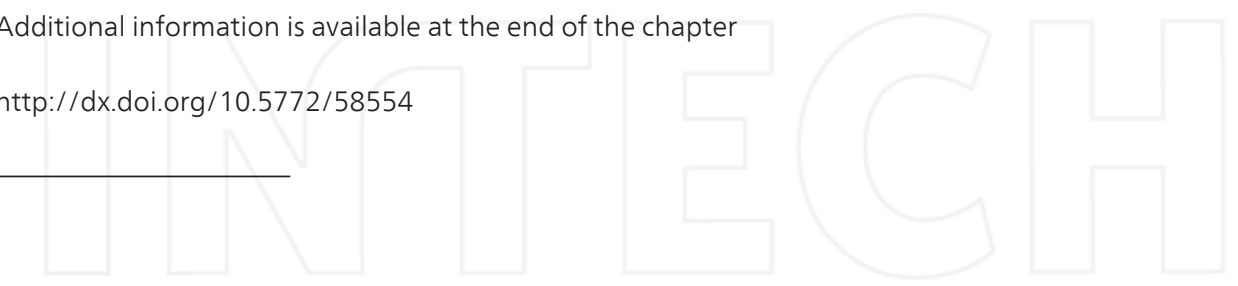

\section{Introduction}

Luminescence dating is a collective term for dating methods that encompass thermoluminescence (TL) and optically stimulated luminescence (OSL) dating techniques. OSL is also less commonly referred to as optical dating [1], photon stimulated luminescence dating or photoluminescence dating [2]. Luminescence dating methods are based on the ability of some mineral grains to absorb and store energy from environmental ionizing radiation emanating from the immediate surroundings of the mineral grains as well as from cosmic radiation. When stimulated these minerals, generally referred to as dosimeters [3], will release the stored energy in the form of visible light; hence the term luminescence. Measuring the energy and determining the rate at which the energy accumulated allows an age representing the time that has elapsed since the energy began accumulating to be determined. Stimulation of energy release using heat is termed TL while stimulation using light is referred to as OSL. The age range of luminescence methods generally spans from a few decades to about 100,000 years, though ages exceeding several hundred thousand years have been reported in some studies [for example, $4,5]$. In addition, there are dating protocols that are currently under investigation that, if successful, could extend the range even further [6]. Thus, the method is useful for dating Late Quaternary events and, not only does it provide chronology beyond the range that can be attained using radiocarbon methods, but it offers an alternative chronometer in settings where no carbon bearing material can be found.

This chapter aims to acquaint readers who are not familiar with luminescence dating methods with the basics of the techniques. It is not intended to be used as a manual but rather as an introductory primer that brings awareness about the principles behind the dating methods, their practical aspects, as well as their applications. Accordingly, the chapter comprises nine sections. Following the introduction in the first section which briefly lays out the historical development of luminescence dating, the second section examines the principles of the dating methods. This is followed by a discussion of sample stimulation mechanisms and basic 
measurement equipment used in luminescence dating in the third section. Luminescence properties of different minerals are examined in the fourth section. In the fifth section, methods used to determine the energy stored within mineral grains (paleodose) as well as the rate at which the energy accumulates (dose rate) are explored. In the sixth section, practical aspects pertaining to sample collection and laboratory preparation for analysis are discussed after which the types of materials that can be dated using luminescence materials are examined in the seventh section. To illustrate the multifaceted character of some luminescence dating studies, the eighth section presents a case study that uses the chronology of postglacial eolian dune deposition in western Canada to constrain the timing of Late Pleistocene deglaciation in the region. The chapter concludes in the ninth section with a look at current and potential future developments in luminescence dating.

\subsection{The historical development of luminescence dating}

The history of the development of luminescence dating spans the last six decades and it began with experimental applications of the phenomenon of thermoluminescence, which is the emission of light when materials are heated to temperatures below those of incandescence. One of the earliest documented suggestions of the possibility of using thermoluminescence to measure time in archaeology was by Daniels et al. [7] who in 1953 proposed using thermoluminescence observed from ancient pottery artifacts that had previously been fired as a measure of their age [8]. A few years later, the application of thermoluminescence to date pottery was discussed by Kennedy and Knopff [9] and technical aspects of measurements that would be employed for dating were described by Grögler et al. [10]. Notably the application of thermoluminescence in geology to study ages of carbonates [11] and lava flows [12] was already being discussed. An early study that tested the application of thermoluminescence dating on pottery was reported by Aitken et al. [8] when in 1964 they applied the method on pottery sherds ranging in age from around 1,000-8,000 years and collected from sites spread over a large area. Results indicated that the luminescence ages were linearly proportional to radiocarbon chronology from contemporaneous materials. Subsequently, developments throughout the rest of the 1960s and the 1970s saw improvements in dating procedures that used thermoluminescence in archaeological applications [3].

Incidentally, parallel developments in the former Soviet Union during the late 1960s and early 1970s began seeing the tentative application of thermoluminescence dating on unburnt Quaternary sediments [13,14] when it was noted that older sediments returned higher TL signals than younger ones. Dating of unburnt sediments was based on the recognition that stored energy in sediment grains could also be depleted by exposure to sunlight as opposed to heating that occurs in fired artifacts. In the West, attempts to apply thermoluminescence on unburnt sediments appear to have begun around the end of the 1970s [15] including attempts by Wintle and Huntley $[16,17]$ to apply the technique on deep sea sediments. Accurate sediment ages from TL dating, however, remained elusive since the optimal conditions for solar resetting were not yet fully understood [18]. Huntley [18] conducted some of the earliest studies investigating the most appropriate conditions for solar bleaching. In a related development, sediment dating using luminescence methods progressed rapidly when it was noted 
[1] that light could also be used to stimulate energy release from sediments during measurement as opposed to heating. This led to the birth of OSL dating and throughout the rest of the 1980s [19, 20], the 1990s [21-25] and the early part of the following decade, improved protocols were introduced $[26,27]$ ) and OSL dating equipment refined. All these developments saw luminescence dating emerge as a robust method for dating clastic Quaternary sediments, especially eolian deposits. A comprehensive historical account of the first 50 yrs of luminescence dating is provided by Wintle [28].

\section{Principles of luminescence dating}

\subsection{Luminescent materials}

Some dielectric materials (insulators) that include many minerals such as quartz, feldspar, zircon and calcite have the ability to store energy in their crystal lattices that emanates from ionizing radiation. In natural geological settings, such ionizing radiation (for example, alpha, beta, and gamma radiation) occurs naturally within the immediate surroundings of the geological materials while a small component is also contributed by cosmic radiation. When stimulated, the minerals will exhibit luminescence which essentially represents a release of the stored energy. Luminescence dating employs this phenomenon by measuring the energy stored in the mineral, called the paleodose, and dividing it by the rate at which the energy was received by the mineral in question. Hence, the basic age equation for luminescence dating is:

$$
\text { Luminescence Age }=\frac{\text { Paleodose }}{\text { Dose rate }}
$$

\subsection{Electron trapping mechanisms}

The exact mechanisms through which luminescence energy accumulates in the minerals are complex. However, it is thought that the energy is stored when electrons in the mineral crystal lattices are displaced from the valence band of their parent nuclei. Once detached, the electrons diffuse into the surroundings of lattice defects that act as electron traps. Such defects include a missing atom in the crystal lattice of the mineral, an atom out of its rightful place or the occurrence of impurity atoms in the lattice [29]. Importantly for dating purposes, the number of trapped electrons increases with the duration of exposure of the mineral to the ionising radiation. Fig. 1 is a depiction of an energy level diagram that is commonly used to visualise the trapping mechanism involved in luminescence. The depth of the trap $(\mathrm{T})$ below the conduction band, indicated by ' $E$ ' [Fig. 1], is an indication of the efficacy of a given trap. Stable traps are those that can withstand perturbations such as lattice vibrations that could dislodge the electrons from their traps. Stimulation of the crystal lattice structure by heating to an appropriate temperature or by optical means using a suitable wavelength will excite the electrons out of the traps. Once expelled from the traps, the electrons diffuse within the crystal lattice until they come across another site that is attractive to electrons and these are referred to as recombination centres [29]. When electrons reach some recombination centres, energy is 
emitted in the form of light and these are described as luminescence centres. Thermal stimulation would yield TL whereas optical stimulation would produce OSL. Importantly, the diffusion process is very rapid such that the time between stimulation and recombination can be treated as instantaneous. For a recombination centre to be effective, an electron must be missing from the site in the lattice, creating what is termed a hole. Holes are created in materials by ionising radiation. The intensity of the luminescence given out following stimulation is proportional to the number of trapped electrons and this is assumed to be proportional to the energy absorbed from the nuclear radiation [29]. Significant for dating applications, however, though the energy storage mechanisms might be the same for a given mineral, the sensitivity to radiation will vary between samples; a consideration that has huge implications for methodological approaches as will be shown later.

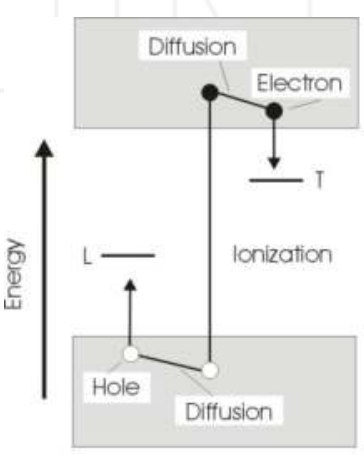

(a) Irradiation

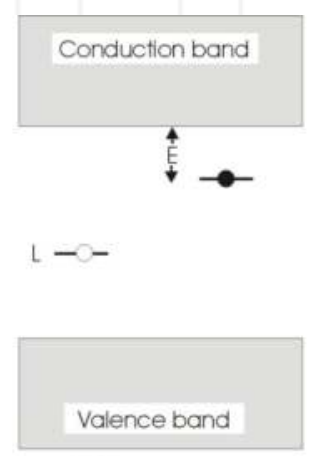

(b) Storage

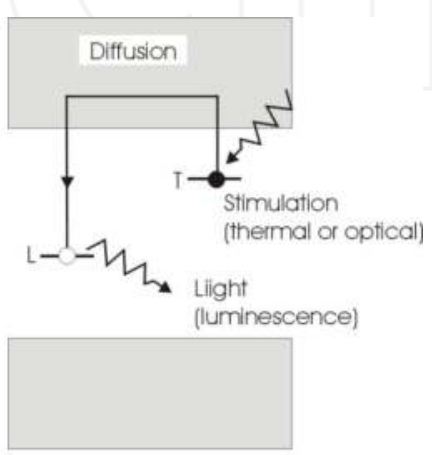

(c) Eviction

Figure 1. Energy Level diagram illustrating the creation of luminescence centres in crystal lattices through exposure to ionising radiation (redrawn from [29]). (a) Irradiation leads to electrons being expelled from their original site and diffuse within the lattice. (b) Electrons become trapped while holes of missing electrons become localised at particular centres. (c) Stimulation (by heat or light) results in electrons being evicted from the traps and diffusing until they meet a recombination centre. Light (luminescence) is emitted when luminescence centres are encountered by the electrons to give TL or OSL.

It is pertinent to note that the number of traps within the lattice of any dosimeter is not infinite and, hence, they will be exhausted following extended exposure to radiation, beyond which energy will not be stored efficiently. This is referred to as saturation. For dating purposes this is what defines the upper limit on the age beyond which samples cannot be dated using luminescence methods [29]. The exact age representing this upper limit will ultimately depend on the dose rate, with samples experiencing high dose rates having lower age limits.

\subsection{Natural sources of ionizing radiation}

For purposes of luminescence dating, natural sources of ionising radiation that contribute to the trapped energy in mineral grains are isotopes of uranium $\left({ }^{238} \mathrm{U}\right.$ and $\left.{ }^{235} \mathrm{U}\right)$ and thorium $\left({ }^{232} \mathrm{Th}\right)$ decay chains, potassium $\left({ }^{40} \mathrm{~K}\right)$ and rubidium $\left({ }^{87} \mathrm{Rb}\right)$. These elements occur in natural materials in very low levels (around 3-10 parts per million for uranium and thorium and generally less 
than $5 \%$ for potassium, where ${ }^{40} \mathrm{~K}$ is one part in 10,000). However, collectively, their radioactive isotopes emit enough radiation to cause detectable luminescence for dating purposes. The radiation emitted includes alpha and beta particles as well as gamma radiation. Alpha particles have penetration ranges of $0.02 \mathrm{~mm}$ while beta and gamma rays have ranges of around 0.02 $\mathrm{cm}$ and $20 \mathrm{~cm}$ respectively [29]. The shorter distance travelled by alpha particles is because they are heavier and much more ionizing, which results in more rapid loss of energy as they knock atoms of materials through which they are traveling out of the way. Beta particles and gamma rays, on the other hand, tend to get scattered.

Besides the radiation from radioactive isotopes, an additional, albeit smaller, component to the energy received by the mineral grains is contributed by cosmic rays from outer space. The cosmic radiation comprises a soft and a hard component and once it reaches the earth's surface, the soft component is absorbed by the upper $50 \mathrm{~cm}$ of the near-surface substrate. Only the hard component can penetrate deeper and is of interest to luminescence dating. This hard component comprises muons mostly and at sea level it varies slightly with latitude, increasing in intensity by about $7 \%$ from the equator to latitude $40^{\circ}$. Above $40^{\circ}$ latitude however, it remains constant up to the poles [29]. At altitudes higher than $1 \mathrm{~km}$, the contribution from the hard component also increases significantly with both latitude and altitude. For dating purposes, special formulae have been developed for evaluating cosmic ray contribution to the dose rate [30].

\section{Basic luminescence measurement equipment and sample stimulation mechanisms}

The primary objective of TL or OSL measurements in dating studies is to ascertain the amount of energy that has accumulated in the mineral grains since the start of the event being dated. This energy is determined by stimulating the mineral grains using an appropriate mechanism and measuring the amount of light released. As already outlined, trapped electrons in luminescence dating can be evicted from their traps by heating, as is done in TL, or using light, in OSL methods. Fig. 2 illustrates the basic layout of equipment used to measure the luminescence. Typically, samples are placed on discs about $1 \mathrm{~cm}$ in diameter and then introduced into the machine in multiples on an appropriate sample holder. A servo-control mechanism moves the sample to the appropriate position for stimulation and measurement. Most modern luminescence measurement systems possess both thermal and optical stimulation capabilities. The luminescence signal from the sample is captured by the photomultiplier tube after going through optical filters.

For TL measurements, the filters help exclude infrared signals from the heating while allowing blue or violet signals. For OSL measurements, the filters reject wavelengths used for stimulation while usually allowing violet and near-ultraviolet wavelengths. The end of the photomultiplier tube closest to the sample is fitted with a photocathode that emits electrons when struck by light photons as a result of the photoelectric effect. Potential differences allow emitted electrons to be attracted by the first dynode in the photomultiplier. For each electron arriving 
at the dynode, several are emitted and the process is repeated through the photomultiplier many times such that several millions of electrons reach the anode at the other end of the photomultiplier for each electron leaving the photocathode. These electrons give an easily detectable current pulse at the anode that is commensurate with the arrival of photons at the photocathode. The current pulses are then amplified and the output is presented as photon counts, representing the luminescence signal. As will be shown below, TL output is distinctly different from OSL output.

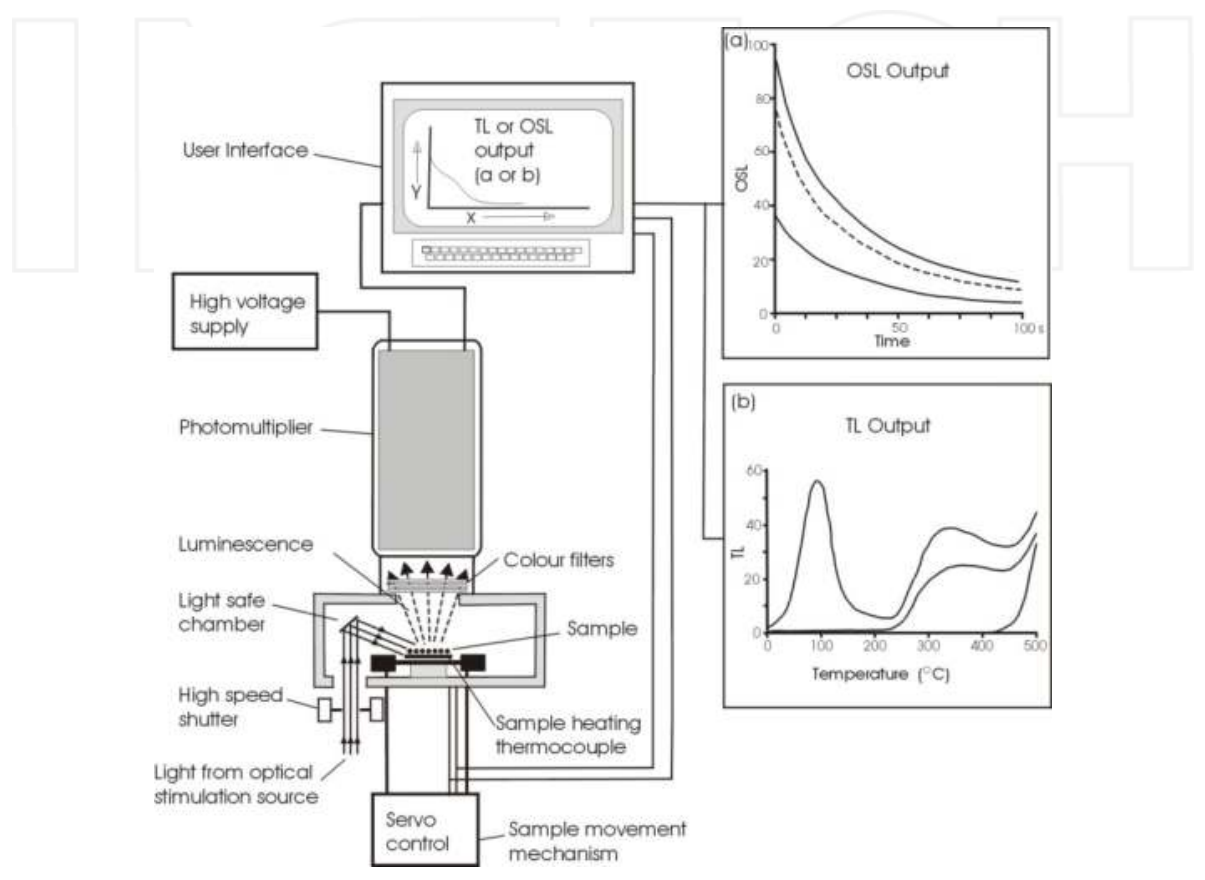

Figure 2. Basic features of a TL/OSL reader that can be used to measure luminescence signals using either heat or optical stimulation (modified after [31]).

\subsection{Stimulation by heat (TL)}

When stimulating using heat, a sample is heated rapidly at rates in the range of $20^{\circ} \mathrm{C} / \mathrm{s}$. Once a temperature commensurate with ' $\mathrm{E}$ ' in Fig.1 and characteristic of a particular trap type is reached, electrons are rapidly evicted from the traps [29]. The temperature is represented by a peak in emission on a plot of the luminescence versus temperature referred to as the TL glowcurve. Continued heating will empty the traps and the luminescence given out will be proportional to the number of electrons trapped in the mineral grains since the beginning of the event being dated. Fig. 3 shows a glow-curve of a sample observed after the first heating. If a sample is heated for a second time immediately after the first heating, a different curve is observed. This second curve is the red-hot glow that is the incandescence given out by any 
material when heated to a temperature high enough. Absent from this second heating will be the luminescence emanating from ionizing radiation that accrued since the last event that emptied the electrons from the traps.

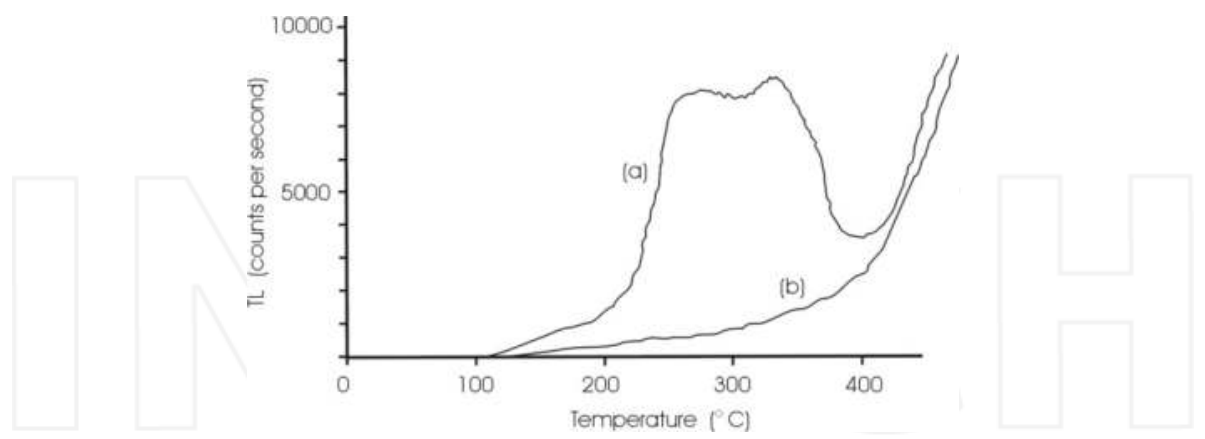

Figure 3. Examples of TL glow-curves showing (a) natural signal obtained from a mineral sample (quartz) during the first heating and $(b)$ red-hot glow-curve obtained from a second heating (incandescence). Note that in curve (a), incandescence is also obtained during the first heating when the sample is heated above $400^{\circ} \mathrm{C}$ (redrawn from [31].

\subsection{Optical Stimulation (OSL)}

Optical stimulation of luminescence uses light of a particular wavelength (for example, blue, green, or near-infrared) to expel electrons from their traps. Notably, the rate at which these electrons are evicted directly depends on the rate at which the stimulating photons are received. The sensitivity of a given trap-type to photostimulation is also an important factor influencing the rate at which electrons leave their traps. In essence, the curve that depicts the emission follows an exponential decay (often referred to as a shine-down curve), with high emission rates in the beginning that gradually fall with continued stimulation (Fig. 4). If the process is continued, at a certain point, all electron traps that are sensitive to optical stimulation become exhausted. Integrating the number of photons released over the period of stimulation quantifies the luminescence of the sample and this should be commensurate with the sample's age [29].

Factors that influence the sensitivity of a trap type to electron eviction by light include characteristics of the trap as well as the wavelength of the stimulating light. Generally, shorter wavelengths are associated with faster eviction rates. Stable traps may require more energy than that available from some optical stimulation wavelengths and in such cases, thermal assistance can be used to bridge the gap. This enables longer wavelengths to be used for stimulation in cases where they would not be able to unassisted [29].

When selecting a stimulation wavelength for OSL measurements, it is important to select a wavelength that effectively allows separation to be made between the wavelength of the stimulating source and that of the emitted signal. As described earlier, filters are used to assist this process. Quartz and feldspar for instance, have strong emissions in the near-ultraviolet $(365 \mathrm{~nm})$ and violet $(410 \mathrm{~nm})$ respectively and filters used in either case are selected because 
they have windows in the respective wavelength range. Wavelengths used for stimulation, therefore, should be excluded by the filters [29].

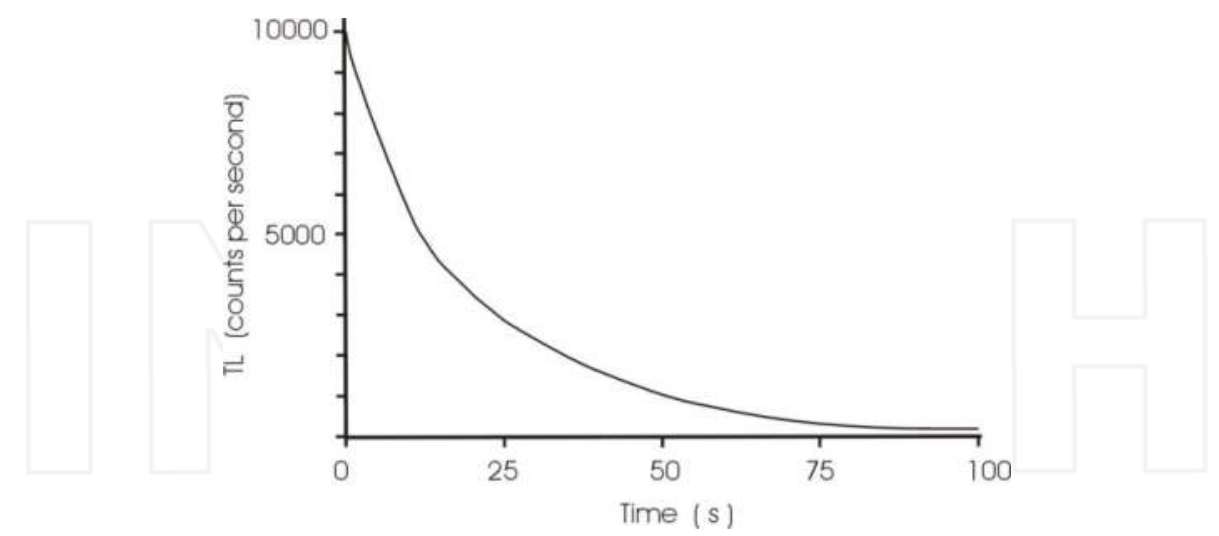

Figure 4. OSL shine-down curve for a hypothetical mineral stimulated using light for about $100 \mathrm{~s}$ (modified from [29]).

\subsection{Advantages of OSL over TL}

As will be shown later, advantages of OSL dating versus TL dating mainly apply to dating of sediments that have been zeroed by solar bleaching. Studies have demonstrated that solar bleaching of natural TL occurs much more slowly than OSL [29].

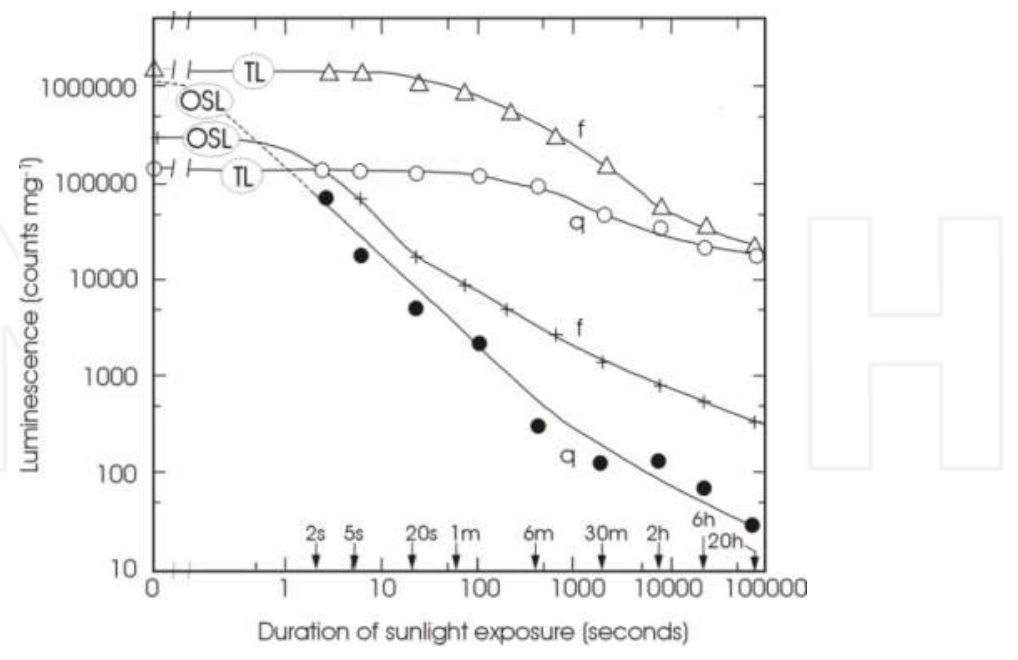

Figure 5. Comparison of bleaching rates of natural TL and OSL (green light) signals of quartz (q) and feldspar ( $f$ ) conducted by Godfrey-Smith et al. [19] (redrawn from [29]). The slower bleaching curves are from the TL signals. 
In a study examining the bleaching rates of quartz and feldspar (Fig. 5), it was shown [19] that after $20 \mathrm{hrs}$ of exposure to sunlight, samples of both minerals had less than $0.1 \%$ of their original OSL signal remaining (Fig. 5). Conversely, similar samples had TL signals that were at least 10 times higher remaining after an equal time under solar radiation. This means that the optical signal is zeroed much more rapidly by the sun and the hard to bleach TL traps generally result in a much higher residual signal following solar bleaching compared to the OSL signal. Consequently, it is often difficult to date very young samples using TL [1, 29]), with OSL being preferred for dating sediments in general. However, dating of fired artifacts and baked sediments using TL remains an appropriate methodology since the zeroing mechanism in nature will be similar to the stimulation mechanism at measurement.

\section{Luminescence properties of some common minerals}

The discovery of luminescence in minerals is not a recent event. Aitken [2, 29, 32] has often cited the case of Robert Boyle who in 1663 reported holding a diamond close to his body and noting that it 'shined in the dark'. This is because many minerals are capable of luminescing in the dark when appropriately irradiated and stimulated. For dating purposes using luminescence methods, however, quartz and feldspar are the dosimeters that have received the most focus. Attempts have also been made to use zircon and calcite but these are not commonly used for dating because of a number of drawbacks. In this section, the luminescence properties of these minerals are briefly examined.

\subsection{Quartz}

Quartz is a widely used mineral in luminescence dating because of the advantages it offers compared to the alternatives. It is one of the most abundant minerals on the earth's surface, making it ubiquitous in most depositional environments, a feature arising from its high stability at the earth's surface and resistance to abrasion. It also has very stable luminescence properties. Because of its chemistry, quartz itself has no internal source of radiation that is a major element of its composition. As a result, the radiation quartz grains receive in nature usually originates from outside the grain. In some settings, however, quartz may contain some trace amounts of uranium [32].

\subsubsection{Quartz TL properties}

For TL analysis, natural quartz signals normally display two peaks above $300{ }^{\circ} \mathrm{C}$ [33]. One peak is at $325{ }^{\circ} \mathrm{C}$ and another at $375{ }^{\circ} \mathrm{C}$ and it is the latter that is usually used for dating. Laboratory irradiated quartz also shows a peak at $110^{\circ} \mathrm{C}$. In terms of emissions, when heated above $300{ }^{\circ} \mathrm{C}$, quartz has a TL emission band in the range $460-480 \mathrm{~nm}$ (blue) and another in the range $610-630 \mathrm{~nm}$ (orange) [34]. Below $300{ }^{\circ} \mathrm{C}$, quartz has an emission in the range $360-420$ $\mathrm{nm}$ (near UV to violet) when irradiated with a laboratory dose. The $375{ }^{\circ} \mathrm{C}$ peak is thought to be from $\mathrm{AlO}_{4}$ impurities in the quartz lattice serving as holes [35].The peak has a high thermal 
stability but it tends to saturate at low doses such that it is of limited use beyond $50-100 \mathrm{ka}$ unless very low dose rates are involved [36].

The $325{ }^{\circ} \mathrm{C}$ TL peak bleaches more rapidly than that at $375{ }^{\circ} \mathrm{C}[36]$ and its emission peaks at about $380 \mathrm{~nm}$ [37]. Hence, by using appropriate filters, the emission can be separated from that of the $375{ }^{\circ} \mathrm{C}$ peak. Studies suggest that, because of its relative stability, the $325^{\circ} \mathrm{C}$ peak could in theory be used for dating up to 1 million years, which is much older than ages that can be measured using the $375^{\circ} \mathrm{C}$ peak [36].

\subsubsection{Quartz OSL properties}

Irradiated quartz has been shown to emit OSL when stimulated by light from any part of the visible spectrum. For dating purposes using current methods, however, blue light is preferred in most applications because, as indicated earlier, OSL yield is proportional to the wavelength used for stimulation, with higher energies yielding higher OSL intensities [38]. The OSL signal from sedimentary quartz has been demonstrated to comprise at least three or four components and these are referred to as fast medium and slow components with respect to the rates at which they decay [39]. Others have reported up to seven components [40]. These components can only be separated when stimulation uses a constantly increasing stimulating power, referred to as linearly modulated OSL (LM-OSL) [41]. A stimulation source with a constant power (continuous wave), as is used in most regular dating, cannot resolve the components. However by using heat treatments (to eliminate unstable signals) or stimulating for controlled times (to exclude dominant slow components) the appropriate signal can be focused on when using continuous wave stimulation.

\subsection{Feldspar}

Feldspar has been used extensively in OSL dating. Like quartz, it is a mineral that is widely available at the earth's surface, though it weathers more rapidly. The chemistry of feldspars has important implications with respect to how they are used in luminescence dating. They are aluminosilicates that have potassium $(\mathrm{K})$, calcium $(\mathrm{Ca})$ or sodium $(\mathrm{Na})$ as end members. The presence of potassium in some of the feldspars is critical in that ${ }^{40} \mathrm{~K}$ isotopes that form part of the potassium constitute an internal source of radiation, in addition to any external radiation the grains may receive. Feldspars that do not have high potassium as part of their chemistry (for example, Ca and Na-feldspars), however, would not have this additional internal dose. Hence, for dating purposes, as will be discussed later, K-feldspars are normally separated from other feldspars prior to conducting analysis.

For dating applications, feldspar has a number of attractive characteristics. One is that, in terms of emissions, feldspars have a higher brightness compared to quartz which means that it gives strong signals, allowing smaller doses to be measured. Secondly, the internal dose in feldspars that have a high potassium content constitutes a reliable radiation source that is immune from environmental changes that would affect external sources (for example, interstitial water). As a result, dose rates can be determined more accurately. The third advantage of feldspar, which will be discussed below, is that it can be stimulated using infrared stimulation. A major 
drawback for feldspar, however, which delayed its application in routine dating, is that it is afflicted by a phenomenon called anomalous fading [42]. In anomalous fading, the measured luminescence intensity decays with increasing time from the time of irradiation because some electrons have much shorter residence times in their traps than predicted by physical models $[29,43]$. The ultimate result is that most feldspar grains will return equivalent doses slightly lower than they would if the dose were stable over time. To address this phenomenon in feldspar dating, correction methods for the fading have been devised [43, 44].

\subsubsection{Feldspar TL properties}

Many K-feldspars of sedimentary origin have been shown to display natural signals with TL peaks at $280^{\circ} \mathrm{C}$ and $330^{\circ} \mathrm{C}$ [36]. With regards to emissions, some studies [for example, 34] have reported emissions from K-rich feldspars in the 390-440 nm range (violet to blue) while plagioclase feldspar emissions have been reported in the 550-560 $\mathrm{nm}$ range (blue-green). However, results from other studies [47] suggest a more complex pattern.

\subsubsection{Feldspar OSL properties}

Luminescence from feldspars has been investigated using visible light stimulation. Earlier investigations used lasers (for example, $514.5 \mathrm{~nm}$ (green) from argon and $633 \mathrm{~nm}$ (red) from krypton) and observed the emissions at shorter wavelengths [1, 19]. For plagioclase feldspars, results showed that the spectra observed were similar to those from TL. Another study [48] that used a stimulation wavelength of $633 \mathrm{~nm}$ also showed that the emission was centred at $400 \mathrm{~nm}$. OSL applications for dating using green light stimulation have been very limited and this has largely been because infrared stimulation (IRSL), as discussed below, was found to be a much better alternative. However, a study [49] that compared green light stimulation luminescence (GLSL) and IRSL data from feldspars from alluvial sediment showed results that suggested the signals had different thermal stabilities, with GLSL signals being more stable than IRSL signals at $10{ }^{\circ} \mathrm{C}$. Stimulation of feldspars using a wide range of wavelengths in the range $380-1020 \mathrm{~nm}$, apart from green and red, has also been demonstrated by [50].

\subsubsection{Feldspar IRSL properties}

Feldspars can also be stimulated using the near infrared part of the electromagnetic spectrum (around $880 \mathrm{~nm}$ ). Since the discovery of this stimulation peak [20], most dating research that uses optical stimulation of feldspars for sediment dating has been focussed on IRSL. A major advantage of this, as stated earlier, is that it leaves the rest of the visible part of the spectrum open for use in emission detection. Other investigations using IRSL include studies on finegrained sedimentary samples containing both plagioclase and K-feldspar that have also shown a major stimulation peak at $854 \mathrm{~nm}(1.45 \mathrm{eV})$ and another weaker one at $775 \mathrm{~nm}(1.6 \mathrm{eV})$ at room temperature [51]. Overall, these characteristics allow feldspars to be stimulated by light emitting diodes that have emission peaks at around $880 \pm 40 \mathrm{~nm}$ and these are widely available and cheap. Emission spectra of the K-feldspars stimulated using IRSL were reported by Huntley et al. [52] to show a dominant peak at $410 \mathrm{~nm}$ and another minor peak between 300 and $350 \mathrm{~nm}$. Plagioclase feldspars, on the other hand, showed an emission peak at $570 \mathrm{~nm}$. 
Krbetschek et al., [53] reported additional natural emission peaks for K-feldspar at $560 \mathrm{~nm}$ as well as at 280 and $700 \mathrm{~nm}$.

Feldspar samples irradiated with a laboratory dose have been shown to display an additional emission peak at $290 \mathrm{~nm}$. For feldspar samples with a natural signal however, this $290 \mathrm{~nm}$ peak is absent. In dating studies, the $290 \mathrm{~nm}$ peak can be eliminated by preheating [36].

\subsection{Calcite}

The mineral calcite has been demonstrated to have a TL signal with an emission at $570 \mathrm{~nm}$ [36] and attempts have been made to use the mineral for dating. However, calcite which often occurs in carbonate cave deposits has a limited environmental occurrence, which constrains its applicability. The luminescence from calcite is also complicated by the tendency of calcite to preferentially concentrate uranium. Thus, evaluation of the dose rate has to account for the disequilibrium of the decay chain of uranium. Notably, calcite dating using the uranium disequilibrium can be used to establish better chronologies than would be attainable using luminescence methods [36]. Hence, overall, the incentive to use calcite in luminescence studies has been low. Published attempts to use OSL emissions from calcite for dating include studies by Ugumori and Ikeya [54]. Nonetheless, as with the TL efforts, these have not translated into widespread applications.

\subsection{Zircon}

Zircon also has luminescence properties. Its properties as a dosimeter are particularly interesting because zircons naturally have a high concentration of uranium such that the internal dose that they receive is usually far greater than any radiation originating from the grain's exterior. As a result, the dose rate is very constant because it is not susceptible to variations that may be induced by changes in interstitial water content or burial depth [36]. A major methodological drawback, however, is that the uranium content is variable between grains and, as a result, measurements have to be made on individual grains. Zircon studies using TL include investigations by Huntley at al. [46] and Templer and Smith [55]. OSL analyses on zircons include studies by Smith [56].

In addition to variations in the uranium content between individual grains, zircon grains also have inhomogeneities in their crystal structures that develop during formation. Hence, for dating purposes, the luminescence from the internal dose is not easily comparable to the signal from the artificial dose administered in the laboratory as would be done with quartz or feldspar. To circumvent this problem, zircon dating often employs the autoregeneration method whereby the natural signal of the zircon is measured after which the grains are stored for several months $[55,56]$. At the end of this storage period, the grains are measured again to determine the signal that has accrued from the internal dose since the initial measurement. This storage signal is then used to calibrate the natural signal from antiquity to determine an age [2]. 


\section{Paleodose and dose rate determination}

As outlined earlier, in order to calculate an age, the basic luminescence age equation divides the dose that has accumulated since the beginning of the event being dated (paleodose) by the rate at which the energy was accumulated (dose rate) (Equation 1). Hence, the two basic parameters that have to be determined are the paleodose and the dose rate. In this section methods used to determine these variables are discussed.

\subsection{Paleodose determination}

Paleodose determination aims to ascertain the amount of energy that has accumulated in a dosimeter since the event being dated occurred. In luminescence dating this is generally the period that coincides with the time when the mineral grains were emptied of any previously accumulated energy (or zeroed). The dose is generally defined as the energy absorbed per kilogram of material and the unit used to measure it is the gray (Gy) where $1 \mathrm{~Gy}=1$ joule per $\mathrm{kg}$. For fired (or heated) materials, the period corresponding to zeroing would be the time when the samples were last heated to the appropriate temperature whereas, for unheated sediments, it would commonly be the time when they were last adequately exposed to the bleaching effects of the sun. The luminescence signal obtained from a sample from the field is referred to as the natural signal. In principle, in order to determine the natural signal in a given sample in Gy, artificial irradiation that is well calibrated is used to induce luminescence in the sample in a laboratory setting after which the natural signal is compared to the signals from the artificial irradiation. This allows the magnitude of the laboratory dose that induces a signal equivalent to that produced by the natural dose to be ascertained. That laboratory dose is referred to as the equivalent dose $\left(D_{e}\right)$. As depicted in Fig. 6, there are two main methods that have been developed for determining $\mathrm{D}_{\mathrm{e}}$ and these are the additive dose and the regenerative dose (or regeneration) methods [3, 29]. Other methods that have been used in the past include the partial bleach method [for example, 17]. However, these are no longer widely used.

\subsubsection{Additive dose method}

As initially developed, to determine the equivalent dose using the additive dose method, samples from the field are typically separated into multiple aliquots. One set of aliquots would have the natural signal measured after which the other aliquots are irradiated with well calibrated incremental doses and then measured, with multiple aliquots being used for each dose level. The acquired signals are then plotted to give a dose-response curve that shows the luminescence signal against the laboratory irradiation (Fig. 6a). This is referred to as a growth curve and it is essentially a simulation of the evolution of the total dose had the sample experienced similar dose levels in its natural setting over time. Since the method employs multiple aliquots, signal normalisation is performed to correct for inter-aliquot variations by giving the aliquots a small test dose afterwards and then measuring the response. Such variations arise from differences in mass and grain sensitivity. To determine $D_{e}$ using the additive dose method, the curve is extrapolated backwards to zero signal intensity and the $\mathrm{D}_{\mathrm{e}}$ will be where the curve intercepts the horizontal (dose) axis (Fig. 6a). In TL dating, the 
residual signal remaining after solar bleaching, would have to be taken into account as well. It is important to note that, for both feldspar and quartz, growth curves usually show a linear relationship between the luminescence signal and the dose in the early part of the curve. If high enough doses are administered, however, the signal tends to level off, indicating saturation or an exhaustion of the luminescence traps.
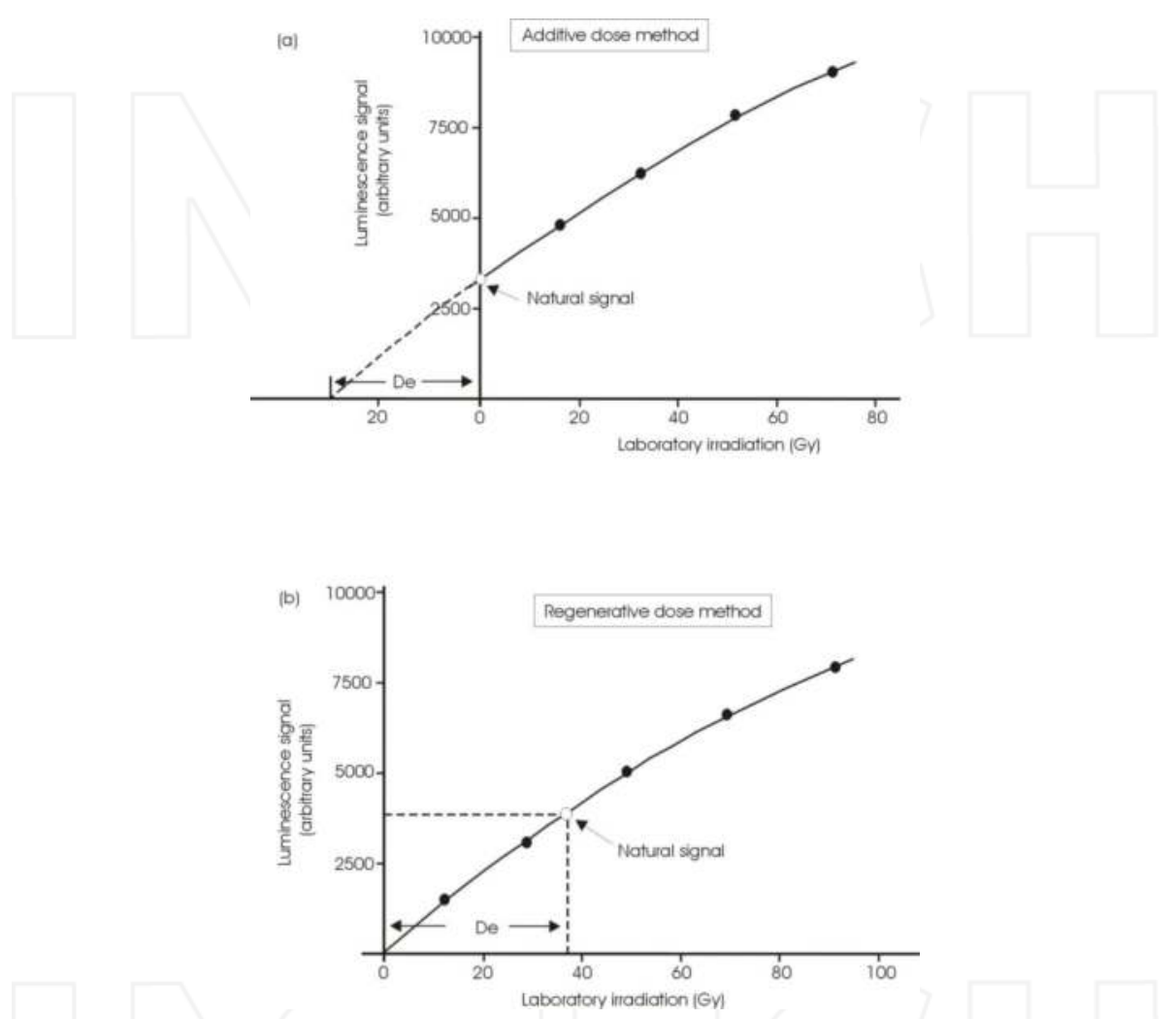

Figure 6. Methods used to determine the equivalent dose $\left(D_{e}\right)$. In the additive dose method (a), incremental doses are given to unbleached samples and measured. In the regenerative dose method (b), on the other hand, the samples are zeroed first before incremental doses are administered and measured (modified after [3]).

\subsubsection{Regenerative dose method}

The regeneration method differs from the additive dose method in that the samples are zeroed first before any laboratory dose is applied. For TL dating, previously fired artifacts, for example archeological materials or baked sediments, are zeroed by heating. For OSL samples, on the other hand, zeroing is achieved by exposure to sunlight. The zeroed aliquots are then given incremental doses as with the additive dose method, preferably with the doses being chosen to lie above and below the natural signal. Normalisation can also be conducted to correct for 
inter-aliquot signal variations. Signals from the doses are then plotted to give a regenerative growth curve. The equivalent dose would be obtained by interpolating the natural signal or the unknown signal into the curve (Fig. 6b).

\subsubsection{Single aliquot methods versus multiple aliquot methods}

As outlined above, when initially developed for TL dating, both the additive dose and regenerative dose methods involved the use of multiple aliquots. With the introduction of OSL dating, though the possibility of using single aliquots was contemplated early [1], the practice of using multiple aliquots was adopted too [36]. An inherent assumption when using multiple aliquots is that all the aliquots of a given sample respond similarly to the dose received. However, this is not what is observed; inter-aliqout variations arise from a number of sources and this necessitates the implementation of normalisation to try and address the differences. Quartz in particular appears susceptible to sensitivity changes. The different grains within any particular aliquot would have experienced dissimilar histories within the natural environment and these can include differences in erosion and deposition cycles, episodes of heating from wildfires or other extreme conditions $[3,57]$. The heating administered in the laboratory as part of the analysis (see further below) also results in sensitivity modifications that are dissimilar between the grains, and ultimately between the aliquots. Variations in sample mass between aliquots may also be a reason for differences in behavior between aliquots. The net result of these disparities is that they give rise to differences in sensitivity that contribute to uncertainties in the calculated ages, even in cases where normalisation is used. Hence, to help address these aspects, there was a desire to develop a method that only utilised a single aliquot. There are a number of advantages associated with the use of single aliquots and these include [36]:

i. when using a single aliquot, to obtain $D_{e}$, the natural signal obtained will be compared to the dose response curve of the same aliquot. Hence, inter-aliquot variations are eliminated resulting in much higher precision for $\mathrm{D}_{\mathrm{e}}$.

ii. only a very small amount of sample material is required. This is particularly important for archeological samples that may be of limited size.

iii. normalisation to correct for inter-aliquot variations in numbers of grains on different sample discs or variations in sensitivity is not necessary when using a single aliquot.

iv. the measurement protocol employed with single aliquots which entails preheating, bleaching, and irradiation can all be conducted within most modern readers which are automated and it increases precision in addition to reducing analysis times.

The following section examines procedures used in single aliquot methods.

\subsubsection{Single-Aliquot Regenerative-dose (SAR) protocol}

The introduction of single aliquot methods began with investigations that employed the additive dose approach. Efforts to use single aliquots for dating quartz had observed that there were sensitivity changes associated with repeated preheating of the aliquot that was required after every successive laboratory dose prior to conducting the luminescence measurements. 
Preheating is necessary because it ensures that the distribution of trapped electrons after administering the laboratory dose is similar to that resulting from the natural dose [58]. To correct for the sensitivity changes using the single aliquot additive dose method on feldspars, Duller [21] monitored an additional aliquot for sensitivity changes. Galloway [22] improved on Duller's [21] approach by correcting the luminescence signals using a least squares fitting approach using measurements made on the same feldspar aliquot subsequent to the additive dose irradiation and measurements. This modification by Galloway [22] essentially transformed the method into a bona-fide single aliquot protocol. Alternatively, using the additive dose method on quartz, Murray et al. [27] devised a correction procedure that included additional preheat and stimulation cycles without any additional dose being given, the results of which were used to formulate a decay constant. The constant was then used to correct the data from the additive dose measurements.

Attempts to use single aliquots with the regeneration method on feldspar [21] and on quartz [58] had initially concluded that it would not be possible because of sensitivity changes [58]. However, to address those sensitivity changes, Mejdahl and Bøtter-Jensen [60] proposed the single-aliquot/ regeneration-added dose protocol (SARA) to date previously heated materials and Murray [61] later used the same method on unheated sediments. However, with SARA, at least two aliquots are required [58]. Subsequently, a truly single aliquot regenerative dose (SAR) protocol was introduced by Murray and Roberts [58] using sedimentary quartz from Australia and, with that SAR method, corrections for the sensitivity changes were made by monitoring the $110^{\circ} \mathrm{C}$ TL signal measured immediately after administering a regeneration dose. A major methodological breakthrough was made when a streamlined version of the method proposed by Murray and Roberts [58] was put forward by Murray and Wintle [26] whereby sensitivity changes were monitored using a test dose whose signal was also measured. As initially proposed by Murray and Wintle [26], this SAR protocol essentially entailed the following sequence:

\begin{tabular}{lll}
\hline Step & Treatment $^{\text {a }}$ & Observed \\
\hline $\mathbf{1}$ & Irradiate sample with dose, $D_{i}$ & - \\
$\mathbf{2}$ & Preheat sample $\left(160-300^{\circ}\right)$ for $10 \mathrm{~s}$ & - \\
$\mathbf{3}$ & Stimulate sample for $100 \mathrm{~s}$ at $125^{\circ} \mathrm{C}$ & $\mathrm{L}_{i}$ \\
$\mathbf{4}$ & Irradiate sample with test dose, $D_{t}$ & - \\
$\mathbf{5}$ & Heat to $160^{\circ} \mathrm{C}$ & - \\
$\mathbf{6}$ & Stimulate for 100 s at $125^{\circ} \mathrm{C}$ & $T_{i}$ \\
$\mathbf{7}$ & bReturn to 1 and repeat sequence & - \\
\hline
\end{tabular}

$D_{\mathrm{i}}$ is the regeneration dose which gives signal $L_{\mathrm{i}}$ whereas $D_{\mathrm{t}}$ is the test dose which gives signal $T_{\mathrm{i}}$. The observed signals, $L_{\mathrm{i}}$ and $T_{\mathrm{i}}$ are then used to plot a curve of $L_{i} / T_{i} v$ s. the regeneration dose, $D$.

aFor the natural sample, $1=0$ and $D_{0}=0 \mathrm{~Gy}$.

bLater modifications stimulate the sample for 40 s (instead of 100s) and an additional step to optically stimulate the sample for 40 s at a temperature above the preheat temperature is conducted after step 6 to reduce recuperation [see 26, 62].

Table 1. Steps in the SAR protocol as originally proposed by Murray and Wintle [26]. 
Since its original introduction, the SAR protocol has undergone some minor modifications (Table 1) [26, 27] and, over the last 10-15 years, the protocol has emerged as the preferred method for routine dating of both sedimentary and fired materials using both quartz and feldspar. Further methodological details can be found in [26, 27, 62].

\subsubsection{Single grain analysis}

Measurement protocols used for analyzing single aliquots can also be adapted for determining paleodoses using individual mineral grains. Special equipment for loading and analyzing single grains have been developed that allow multiple sand-sized grains (up to $250 \mu \mathrm{m}$ ) to be mounted individually in a regular array that permits automated measurement [25]. In this format, thousands of grains can be analysed in a relatively short period of time. Analyzing single grains makes it possible to recognize differences in behaviour between grains from a given sample. For instance, it enables the identification of grains that have been bleached to different levels prior to burial because such grains will yield different paleodoses. For that reason, single grain analysis is commonly used to identify partial bleaching in sediments, especially in fluvial deposits [63].

\subsubsection{Presentation of luminescence paleodose data}

Once determined, paleodose results can be presented as growth curves as in Fig. 6, with the horizontal axis showing the dose given and the vertical axis showing the luminescence signal (or nor normalised signal). However, for SAR procedures where multiple determinations can be made on the same sample that yield a range of equivalent dose values, individual growth curves do not convey all pertinent statistical information. Alternative means that can be used to provide some statistical information include frequency histograms. However, histograms do not provide information on precision [64]. To address that aspect, paleodose data in luminescence dating are now commonly presented as radial plots that show both the number of $D_{e}$ determinations made as well as the relative precision associated with each determination [65].

In the example of a radial plot given in Fig. 7, each dot represents an equivalent dose that was determined for a single grain using the SAR approach for a total of 204 grains. Had multiple grain aliquots been used, each data point would denote an aliquot. Any straight line that passes radially through the origin represents a line of constant dose. The horizontal axis at the bottom shows the relative error associated with each paleodose calculation, with the precision increasing from left to right. The shaded area in the plot [Fig. 7] denotes the 2 sigma error band centred at the equivalent dose of $25 \mathrm{~Gy}$, representing the weighted-mean of all 204 data points. Thus, the band represents a 95\% confidence level on all aliquots that were analysed. The number of data points that lie outside the shaded area are reflected by the overdispersion of the data and can be calculated [65]. The equivalent dose scale on the right is a logarithmic scale. Overall, radial plots enable investigators to visualize the dose distributions, allowing appropriate data to be targeted for further analysis. For instance, they permit investigators to differentiate between variations in equivalent dose that arise from the bleaching history and local dose rates from those that are caused by intrinsic differ- 


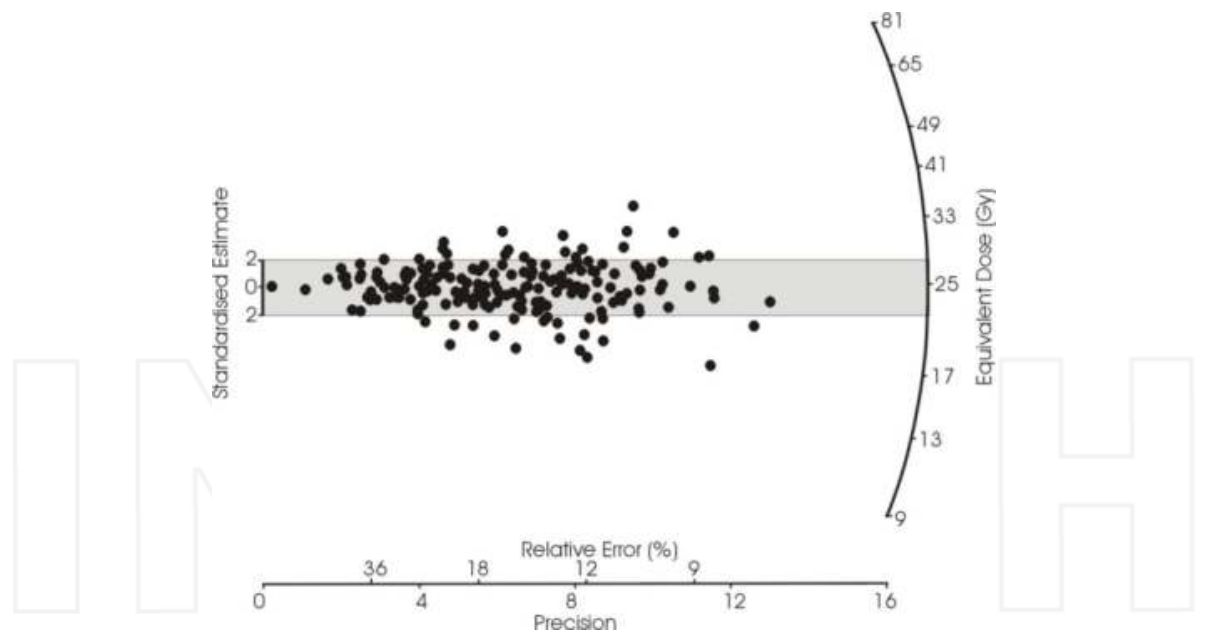

Figure 7. Radial plot of equivalent dose $\left(D_{e}\right)$ estimates for 204 single grains from Sample M3T 61.2 m from Lake Mungo, Australia, obtained using the SAR protocol (redrawn from [66]). Each data point represents a single grain. Precision, shown on the $\mathrm{x}$-axis, is simply the reciprocal of the standard error. Hence, as relative error decreases as one moves to the right, the precision increases.

ences in luminescence sensitivity of the measured aliquots [64]. Accordingly, radial plots have often been used to identify poorly bleached samples, especially when used to analyze individual grain paleodoses. Once the paleodose is determined, statistical models used to calculate the age include the central age model, which calculates the weighted mean equivalent dose from a set of data points taking into account the overdispersion above that associated with measurement errors. Similarly, the minimum age model gives the equivalent dose associated with the population of aliquots (or grains for single grain dating) with the lowest dose. Details on these models can be found in [65].

\subsection{Dose rate determination}

Apart from the paleodose, an additional element that has to be determined before a luminescence age can be calculated is the dose rate. As outlined earlier, the main isotopes responsible for the accumulation of luminescence energy in natural setting are the isotopes of the uranium and thorium decay chains, potassium and rubidium as well as cosmic radiation. Hence, the total contribution of these effects have to be evaluated. There are several methods that can be used to determine the total dose rate. One is the concentration approach and with this method, concentrations of thorium, uranium, potassium and rubidium in representative samples are measured using an appropriate technique such as neutron activation, atomic absorption spectroscopy (AAS), X-ray fluorescence (XRF), flame photometric detection (FPD) or inductively coupled plasma mass spectrometry (ICP-MS). However, measurement of uranium and thorium using this approach can be inaccurate if there is disequilibrium [29]. 
High-resolution gamma ray spectrometry and alpha spectrometry are alternative methods that can be used to measure activities of individual radionuclides, including those from the thorium and uranium decay chains. Hence, they can be used to measure dose rates whether there is disequilibrium or not. However, extended measurement times could be required and the equipment can be costly [29].

Another approach that has been employed to minimise effects of disequilibrium of the thorium and uranium chains is to measure the contribution of uranium and thorium using thick source alpha counting (TSAC) and then use AAS, XRF, FPD or ICP-MS to measure potassium. An alternative procedure is to measure the alpha contribution only using TSAC and determine the beta contribution using a beta particle counter. Whenever possible, the gamma dose rate should be measured on site, especially in settings where there is uncertainty about the uniformity of the dose within a $30 \mathrm{~cm}$ radius of the sample. The recent development of powerful portable gamma-ray spectrometers has made such field measurements relatively practical [67]. Other field measurement options include the use of very sensitive synthetic dosimeters such as $\alpha-\mathrm{Al}_{2} \mathrm{O}_{3}: \mathrm{C}$ that only need to be buried in the field for a few weeks at the most, as opposed to earlier dosimeters that required burial for up to a year [67].

As described earlier, cosmic-rays also contribute to the dose received by the mineral grains. This contribution is usually minor but in settings where the contributions from the radionuclides are low, cosmic contribution can be significant. Formulae for evaluating cosmic ray contribution have been provided by Prescott and Hutton [30].

The effect of moisture content in the natural setting of the material being dated is to absorb part of the dose that should normally reach the grain. Consequently, when calculating dose rates, the levels of moisture content have to be noted and factored into the determination. In essence, dry sediment will experience a higher dose rate than moist sediment.

\subsection{Lower and upper limits for luminescence ages}

Improvements in luminescence dating instrumentation and dating protocols have reached a stage where current OSL methods can be used to date samples deposited as recently as the last few decades [for example, 68]. The single grain dating method in particular can yield dates with very high precision. Prerequisites for dating such young samples include appropriate bleaching to remove all previously acquired luminescence energy prior to burial as well as the availability of grains that have high luminescence sensitivity [28]. To optimize the measurements and increase precision, thermal charge transfer is minimized in order to increase the signal size. Such advances mean that luminescence methods can now produce ages from the last 300 years that are more reliable than those attainable using radiocarbon methods. Calibrated radiocarbon ages from the same period have comparatively larger uncertainties due to fluctuations in ${ }^{14} \mathrm{C}$ production [69].

With regards to the upper age limit attainable using luminescence methods, empirical studies have demonstrated that the storage of luminescence energy through the trapping of electrons is not a process that can continue indefinitely within any given material because, eventually, the traps do get exhausted $[29,31]$. For that reason, luminescence growth curves are often 


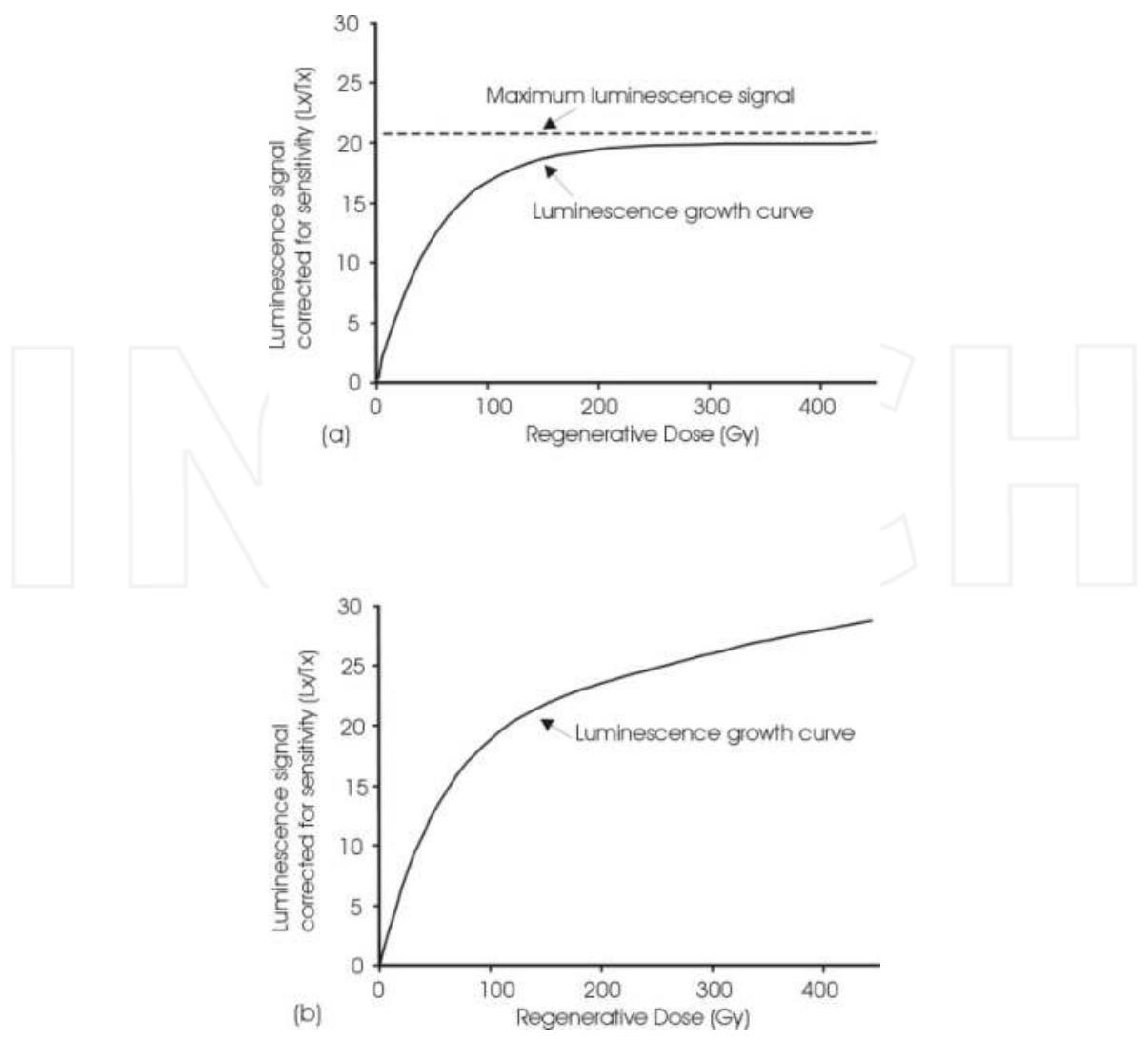

Figure 8. Illustration of quartz growth curves constructed using the SAR protocol (redrawn from [3]). In (a) the curve is constructed using a saturating exponential of the form $I(D)=I_{0}\left(I-\exp ^{-D / D_{0}}\right)$ where I(D) denotes the OSL signal commensurate with the dose $D$ and $I_{0}$ is the maximum OSL intensity that can be obtained. The variable $D_{0}$ determines the shape of the curve and in curve (a), $D_{0}$ is 55 Gy. Because curve (a) appeared to underestimate some older ages, in (b) the saturating function from (a) is combined with a linear function [3].

represented by saturating exponential functions and the form of the curve determines the maximum luminescence signal that can be stored in the mineral grain beyond which no dose can accumulate efficiently. For quartz for instance, Fig. 8a shows the fast component expressed as a single saturating exponential and, beyond a certain dose, the curve flattens. Dating of quartz samples close to saturation ( $>100 \mathrm{~Gy}$ ) has been demonstrated in some studies to yield OSL ages that underestimate the true age by up to $10 \%$ [for example, 70 ]. In reality, the absolute age limit will be determined by the dose rate, with low dose rates having higher age limits. The curve in Fig. 8a also shows that the growth curve is relatively linear for low doses, and ages from the linear part of the growth curve have been shown in numerous studies to be comparable to those obtained using other dating methods, for example, radiocarbon. Generally, however, these results indicate that the storage of luminescence dose in mineral grains 
has an upper limit beyond which the traps become saturated. This dose level places a limit beyond which the method cannot be applied. For quartz this dose is about 100-150 Gy. The solution adopted in some studies when working with older ages $[4,5]$ is to model the luminescence growth using a combination of the saturating function and a linear function to give a curve as in Fig. 8b [28]. Ages in excess of 200-400 ka have been reported from quartz using this method [for example, 5]. Feldspar ages in excess of $100 \mathrm{ka}$ obtained using IRSL signals have also been reported in a number of studies [71]. However, at such dose levels (>100-200 Gy) the growth curve is no longer linear such that correction for anomalous fading using standard procedures [43] becomes problematic.

Overall, however, the indication is that for both feldspars and quartz, there is an upper age limit beyond which accurate OSL ages cannot be obtained. The exact limit will ultimately depend on the dose rate.

\section{Sample collection and preparation: some practical aspects}

Having looked at the principles of luminescence dating and the methodological aspects in the preceding sections, a topic that needs to be discussed are the types of materials that can be dated using luminescence methods. Prior to exploring that topic, however, it is imperative to examine aspects of the dating method that have a bearing on the types of materials one can date using luminescence techniques. Accordingly, this section examines the importance of grain size after which sample collection and preparation methods are discussed.

\subsection{Importance of grain size}

For technical reasons, luminescence dating is usually conducted on mineral grains in two broad grain size categories: coarse grains and fine grains. In the coarse grain method, grains in the fine sand category are separated and analyzed. Such grains would normally receive dose from alpha, beta, and gamma radiation. However, because alpha particles can only penetrate the outer $25 \mu \mathrm{m}$ of the grain, the coarse grains are typically etched using hydrofluoric acid (HF) to remove the outer ring of the grain that experienced alpha radiation. As a result, methods that date coarse grains are often referred to as inclusion dating methods [33]. For quartz inclusion dating of pottery, particles in the size range $90-125 \mu \mathrm{m}$ are usually extracted [2]. For sediment dating, on the other hand, coarse grains representing the modal grain size are normally extracted. For eolian dunes for instance, grains in the size range 150-180 $\mu \mathrm{m}$ are usually preferred [3]. In feldspar inclusion dating, similar procedures are used to extract grains in the fine to medium sand size range and etching can also be used to remove alpha particle effects. As a result, when dating coarse grains, the annual dose is calculated by evaluating contributions from beta, gamma and cosmic radiation and the age equation is modified to:

$$
\text { Luminescence Age }=\frac{\text { Paleodose }}{0.90 D_{\beta}+D_{\gamma}+D_{C}}
$$


where $\mathrm{D}_{\beta}, \mathrm{D}_{\gamma}$ and $\mathrm{D}_{\mathrm{c}}$ refer to the beta, gamma and cosmic ray dose contributions respectively $[29,31]$. When the dose rates are expressed as annual rates, the age will be given in years. The beta contribution in Equation 2 is factored by 0.9 to account for the attenuation due to grain size as well as the etching that removes some parts of the grain that received the beta dose [29, 31]. Exceptions to Equation 2 are only those cases where the quartz itself has some uranium and thorium within it. Also for feldspar dating, where K-feldspars are usually isolated from Na-feldspars and dated separately, an additional parameter would also have to be included in the denominator in Equation 2 to account for the internal dose from potassium.

In fine-grain dating procedures, dated materials are often not separated into mineral specific concentrations. Rather, polymineral grains with diameter in the range $4-11 \mu \mathrm{m}$ (fine silt) are extracted and analysed. Because of their size, alpha particles can penetrate these particles completely and alpha contribution has to be taken into account when calculating the dose rate. As a result, general age equation is modified to:

$$
\text { Luminescence Age }=\frac{\text { Paleodose }}{\mathrm{k} D_{\alpha}+D_{\beta}+D_{\gamma}+D_{C}}
$$

where $\mathrm{D}_{\alpha}, \mathrm{D}_{\beta}, \mathrm{D}_{\gamma}$ and $\mathrm{D}_{c}$ refer to the alpha, beta, gamma and cosmic ray dose contributions respectively. Alternatively, in some studies, fine grained quartz is extracted from the polymineral mixture of fine grains using procedures outlined below and analyzed separately. There are some studies that have used intermediate size grains in the range 35-63 $\mu \mathrm{m}$, which is coarse silt [for example, 72]. For such studies, alpha particle contribution also has to be taken into account when calculating the dose rate.

\subsection{Sample collection and laboratory procedures}

\subsubsection{Sample collection}

When collecting samples for dating using luminescence methods, a primary requirement that has to be fulfilled is that the mineral grains to be analyzed should not be exposed to light from the time they are initially buried up until the point they are exposed to the stimulating source during measurement. This restriction necessitates the adoption of special precautions during sample collection and a number of procedures have been devised over the years. For archaeological artifacts this may entail extracting material from the interior of the artifact using a drill under safe light conditions. For sediments, however, measures taken include sampling at night $[29,64]$. Not only is this an inconvenient method because of the need to work in the dark, but there is a greater risk of accidentally exposing the sample to light during the collection [73]. For sediments that are adequately firm, an alternative approach is to cut out a block of sample from the depositional unit being investigated and transfer it to the laboratory where a portion for analysis is extracted from the sample's interior [29, 64]. A sampling approach that has become a method of choice because of its ease and relative guarantee for retrieval of an unadulterated sample is to insert an opaque pipe made of metal, PVC or ABS plastic into a freshly prepared profile face [29]. Once retrieved, the pipe is immediately capped on both ends with an opaque and 
preferably airtight seal. At the laboratory, sediment at the ends of the pipe is removed and the sample for luminescence measurements is taken from the central portion of the pipe. In places where depositional units are not directly accessible, drilling has also been used to reach targeted units and methods that can be used for such sampling are reviewed in [73].

\subsubsection{Sample preparation prior to measurement}

Once the collected sample has reached the laboratory, it has to be prepared for analysis and a number of procedures have been established depending on the material targeted for analysis. As outlined in Section 6.1, samples for luminescence analysis are either measured as fine or coarse grains. It was also indicated that for coarse grains, analysis is usually made on pure mineral separates (for example quartz, or K-feldspars) whereas for fine grains, either polymineral fractions or fine grained quartz extracts can be used. When dating fine grained sediments such as loess, for instance it would be preferable to use the fine grain procedure whereas for eolian dune sands, the coarse grain approach would be more appropriate. In sediments that have equal components of fine grains and coarse grains, dating both fractions would provide a good mechanism for comparison as a cross-checking method.

For coarse grained materials, quartz or feldspar are typically separated using a heavy liquid such as sodium polytungstate solution. Fig. 9 is a flowchart of a separating procedure for quartz and feldspar using heavy liquids with successively lighter or heavier specific densities. Prior to separating minerals using the heavy liquid, carbonates and organic materials that often occur in sediments, and are usually introduced during the postdepositional phase of the deposit, are removed using hydrochloric acid and hydrogen peroxide respectively. Additional information on separation procedures of coarse grains can be obtained from [74, 75].

For fine grains, carbonates and organic materials are also removed using dilute hydrochloric acid and hydrogen peroxide respectively. Dilute sodium oxalate solution is then added to prevent flocculation of the particles after which appropriate grain sizes are separated using a sedimentation column. Sedimentation columns employ Stoke's law which states that the velocity of a particle's sedimentation in a fluid also depends on the size of the particle. Hence, by extracting sediment from the column after a predetermined time following agitation permits a desired grain size to be isolated. As indicated earlier, while fine grains can be analysed as polymineralic fractions, some studies extract pure quartz from the fine grains by digesting the feldspars using fluorosilicic acid [for example, 76]. Detailed information on separation procedures of fine grains for luminescence dating have been provided by [77].

Once separated, coarse grains (quartz or feldspar) or the fine grains (mixed polymineralic or pure quartz) are mounted on appropriate sample discs prior to analysis. Typically, the measurement discs are made of stainless steel or aluminum and measure around $10 \mathrm{~mm}$ in diameter and $0.5 \mathrm{~mm}$ thick [29]. To mount coarse grains, a monolayer of the sample is deposited on the disc in dry form. Silicone oil can be used to help the sample grains adhere to the disc. For fine grain particles, on the other hand, once the desired grain sizes have been isolated, these are mounted on the discs in a solution of acetone, ethanol or water which is then allowed to evaporate, leaving the sediment deposited on the disc. In either case, the disc will now be ready for analysis. 


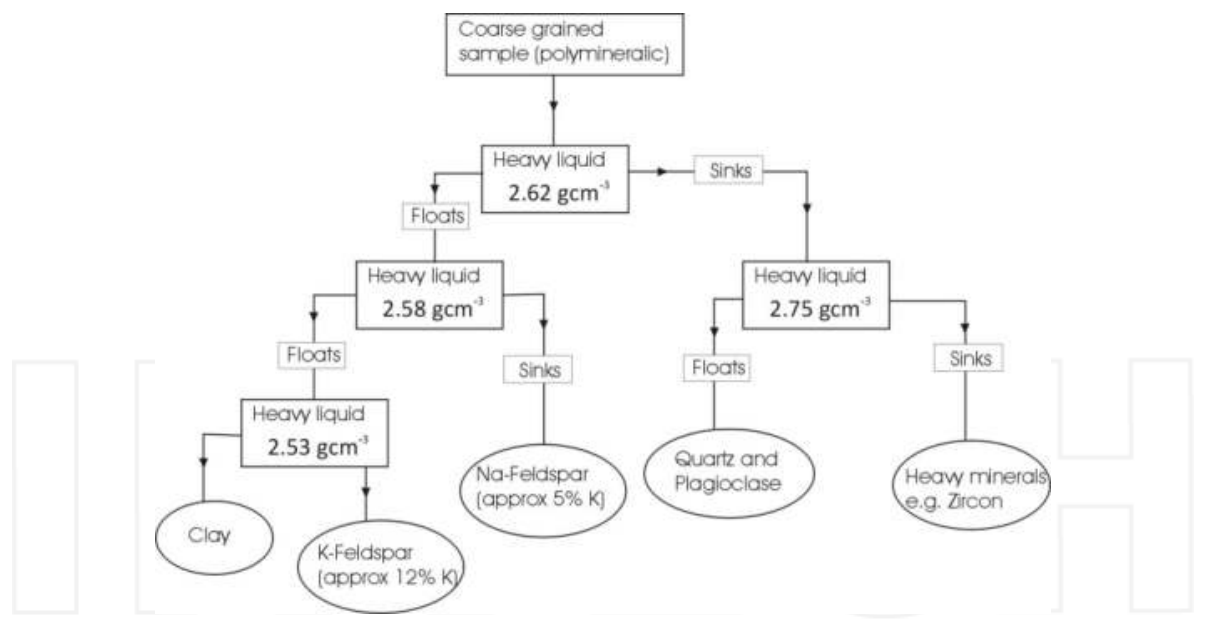

Figure 9. Separation procedure for coarse grains using a heavy liquid. Starting off with a liquid of specific gravity (s.g.) $2.62 \mathrm{gcm}^{-3}$, the sample is separated by centrifuge into a fraction that is lighter (floats) and one that is heavier (sinks). The fraction that sinks is passed through a liquid of s.g. $2.75 \mathrm{gcm}^{-3}$ and the fraction that floats will comprise mostly quartz or plagioclase while the sinking fraction will comprise heavy minerals, for example, zircon. The fraction that floats in the liquid of density $2.62 \mathrm{gcm}^{-3}$ is passed through another liquid of s.g. $2.58 \mathrm{~g} \mathrm{~cm}^{-3}$. The fraction that sinks will be Na-feldspar whereas the lighter fraction is further separated using a liquid of s.g. $2.53 \mathrm{gcm}^{-3}$ into K-feldspar and clay.

\section{What materials can be dated using luminescence methods?}

For all practical purposes, an important aspect about luminescence dating is knowing what type of materials or sediments can be dated using luminescence methods. Discussions in the preceding sections have touched on this topic albeit indirectly. As detailed earlier, luminescence methods date materials by measuring energy that has accumulated in materials called dosimeters. Hence, one prerequisite for dating using the method is that the material to be dated must contain a dosimeter. The second prerequisite is that there has to be an event that reset the energy previously stored by the dosimeter to provide a starting point for counting the time. In essence, all energy stored in the dosimeter will be assumed to have accumulated since that point. A third requirement for material to be datable using luminescence methods is that the electron traps in the dosimeter should not be exhausted at the time of dating because once energy storage has reached a point of saturation, the relationship between time and dose rate breaks down. Hence, the sample for dating has to be younger than the upper age limit that can be attained for that particular dosimeter and, as indicated earlier, the exact limit ultimately depends on the dose rate too. With these considerations in mind, materials datable with luminescence techniques broadly fall into two main categories: materials that have been heated and clastic sediments of sand and silt size that have been reset (zeroed) through exposure to solar radiation. 


\subsection{Heated materials}

As discussed in an earlier section, luminescence dating initially began as an archaeological technique that was used for dating materials that had been heated to temperatures adequately high (for example, $>500^{\circ} \mathrm{C}$ ) to expel all electrons from their traps. Hence, the heating process provides a starting point that can serve as year zero when dating such artifacts. Fired artifacts such as ancient pottery, tiles, bricks or terracotta figures are all examples of archeological materials that can be dated using luminescence methods, especially TL, because these materials usually contain dosimeters such as quartz and feldspar. By separating the quartz and dating quartz inclusions, or by using the fine grained components to date polymineralic fractions or quartz separates, ages can be obtained. Humans are generally believed to have discovered the art of making pottery during the Neolithic period, which dates back to about $30000 \mathrm{yrs}$ ago in some places [for example, 78], but the art only became widespread about 10000 years ago [32]. Hence, unless dose rates are very high, most ancient pottery artifacts are not yet saturated and should be datable using luminescence methods.

Apart from pottery, other heated materials of archaeological significance that can be dated using luminescence methods include burnt flint and burnt stones that were associated with human settlements and may have been heated to high temperatures. Flint is a sedimentary form of quartz and hence possesses dosimetric properties [32]. Paleolithic humans used flint extensively as a tool for scraping and for cutting as well as for projectile points. Chips or debitage left over from the manufacture of such implements can be found associated with ancient settlements. If any of these tools or chips were at some stage heated, either deliberately or accidentally during the occupation of the site by humans, dating the objects using luminescence methods will provide a chronology commensurate with the timing of human habitation. Thus, heated flint can be a useful chronometer especially for timescales beyond those commonly covered by other methods such as radiocarbon [32]. Other heated geological materials include stones that were used as 'pot-boilers' by some societies prior to the discovery of pottery. In some settings stones were also used for constructing fireplaces. In both instances, where these previously heated stones contain appropriate minerals they can be dated using luminescence methods [for example, 79].

A different class of heated materials that can be dated using luminescence methods are geological materials that have been heated to appropriate temperatures to have zeroed them during the last approximately 100,000 years. Such materials include contact-baked sediment that is heated to high temperatures following a volcanic eruption. In such cases, the soil can be collected and grains extracted for dating using either coarse grain or fine grain methods [80]. If the sediment contains larger clasts such as gravel, constituent quartz or feldspar grains can be extracted from the pebbles for analysis. The age obtained would be congruent with the timing of the volcanic eruption [32].

Also associated with volcanic eruptions are the products of the eruption itself such as lava and ash. The heat associated with the volcanic eruption is sufficient to zero these products of any previously acquired dose, if at all, since the lava would mostly be in liquid form rather than crystalline state. Fine grained glass $(4-11 \mu \mathrm{m})$ extracted from volcanic ash has been used in 
some studies to date the eruption [81]. Other studies have attempted to use minerals from the lava instead of volcanic ash [for example, 82]

Finally, also related to the effects of heating to reset the luminescence signal are materials that have been zeroed by heat emanating from the impact of a meteorite. The thermal shock associated with such impacts can reach temperatures high enough to zero constituent grains of the affected geological material [for example, 83].

\subsection{Dating of sediments reset by sunlight}

A class of materials that has fostered the rapid development of luminescence dating methods over the last three decades are clastic sediments that have been zeroed by solar bleaching. Since the confirmation by Huntley et al. [1] that energy from sunlight was capable of adequately erasing previously accumulated energy from dosimeters, there have been many applications of the method to obtain chronologies from sedimentary materials, initially using TL and later using OSL. As outlined above, the prerequisites for dating using luminescence methods which include a presence of a dosimeter in the material, the occurrence of a bleaching episode that erases any previously accumulated energy, and the absence of saturation in the dosimeters (sediment grains) need to be satisfied if a material is to be dated. A number of sedimentary materials satisfy these criteria and have been dated using luminescence methods, with some presently constituting formidable chronological archives of environmental change. These include sediments deposited by wind (eolian sediments), water-laid sediment, glacial deposits, and earthquake related sediments. These sediment classes are discussed below under the respective headings. In all instances, reference to ages obtained from sediments bleached by solar resetting denotes burial ages or time that has elapsed since the last time the sediments were exposed to light from the sun.

\subsubsection{Eolian deposits}

Wind deposited (eolian) sediments are the sediment of choice for dating using luminescence methods. This is because the subaerial transport that the sediment experiences during transportation is expected to provide adequate time to bleach the sediment grains of any previously accumulated energy [29]. While this may not be true in some cases, results generally show that in most settings, that assumption is valid [84]. As a result, this class of sediments has provided the majority of luminescence ages reported to date. Eolian sediments generally fall into one of two main classes. One class comprises sand grains in the fine to medium size range $(63-250 \mu \mathrm{m})$ that are generally transported by wind through a series of low jumps along the surface of a sedimentary bed in a process referred to as saltation [85]. These grains are usually deposited as dunes. The other sediment category is silt size grains $(2-63 \mu \mathrm{m})$ that are transported by wind in suspension. Fine silt grains can remain airborne for extended periods of time [85]. For both sand size and silt size grains, the particle transport at the surface is often adequate to zero the grains. 
Quartz and feldspar grains extracted from eolian sediments have been dated in numerous studies which have compared the chronologies obtained to those from radiocarbon ages from associated sediments and have provided results that are congruent, validating luminescence dating of eolian sediments as reliable chronometers [for example, 86]. In many cases, because eolian deposits are proxy indicators of dry conditions from the past, luminescence ages from the eolian deposits have been used to provide a temporal framework for environmental changes from the past. Fossil dunes from inland deserts of Australia [for example 87, 88], southern Africa [for example 88, 89], Mongolia [for example, 90], United States [for example, 91, 92], Canadian Prairies [for example, 93, 94], the coversands of northern Europe [for example, 95], South America [for example, 96] and many other regions have all been dated using luminescence methods. Reported ages range from a few decades to over 100,000 years and, in many ways, luminescence dating has revolutionised the study of the geomorphology and paleoclimates of arid regions over the last three decades [84].

Apart from inland deserts, luminescence dating has also been used to date deposits from coastal dune deposits [for example, 97] where the ages obtained provide a chronological framework for processes in the coastal environment, including sea level change [for example, 98].

Sequences of fine grained eolian sediments (silt size) give rise to loess deposits which can reach hundreds of meters in thickness [85]. Fine grain dating methods have been used to provide chronological frameworks for the deposition of such sediments from places such as the Loess Plateau of China [for example, 99, 100] to the North American Great Plains [101]. Ultimately the results in such studies are used for paleoenvironmental reconstruction too. As indicated earlier, methods that use fine grains can employ IRSL stimulation of polymineral aliquots, which targets the feldspars. Alternatively, quartz from the loess can be extracted and dated using blue OSL stimulation [for example, 102].

\subsubsection{Water-lain deposits}

Sediments deposited by water, either fine grain or coarse grain, have been dated using luminescence methods. The abundance of sand in fluvial systems makes luminescence dating an attractive dating method in such settings. However, it is the case that sediments transported by fluvial processes are not always completely zeroed, such that the grains are often partially bleached $[29,103,104]$. Statistical approaches for dealing with the partial bleaching have been proposed but there is no consensus on how these should be applied. This has led Cunningham and Wallinga [104] to propose a protocol for analysing OSL data from fluvial sediment using a Bayesian approach. Nonetheless, in studies that have investigated fluvial sediments, luminescence chronology has provided information on modern and ancient sedimentation rates [104]. It has also enabled investigators to assess response patterns of river systems to climatic and tectonic forcing [104]. Results from dating of fluvial sediments can similarly be applied to paleoseismic and archaeological studies [103]. Comprehensive reviews of luminescence dating of fluvial sediments can be found in [103, 104]. 


\subsubsection{Sediments of glacial origin}

For luminescence dating purposes, sediments of glacial origin can generally be classified into three broad categories with regards to solar bleaching possibilities [67]. One class of sediments is transported below the glacier where no bleaching occurs at all. Hence this class would not be appropriate for luminescence dating. The second class is transported within the glacier itself where limited bleaching could occur under some circumstances but often does not. The third class of sediment is transported above the glacier and, for such sediment, some bleaching could occur. The greatest opportunity for bleaching of sediment associated with glaciers, however, is noticed in sediment transported from the glacier as outwash materials by meltwaters $[67,105]$. As a result, the likelihood of the luminescence signal being zeroed increases appreciably with distance of transportation away from the glacier [105, 106, 107]. In practice, however, investigators have noted that, even for outwash deposits, partial bleaching is a problem that is encountered frequently [106]. It is thought that this occurs because the transport of sand and fine grained sediment in the proglacial environment occurs in meltwaters that are both deep and turbid such that penetration by sunlight is impeded [105]). Methods that can be used to date such sediments include single grain methods that try to identify individual grains that were adequately bleached. Current research looks at identifying mineral characteristics such as rapidly bleaching components of the luminescence signal that can be used for dating such sediments [105]. Varying dose rates during the burial history of glacigenic deposits can also be of concern and these have to be reconstructed carefully if accurate ages are to be determined [67]. Additional information on dating of glaciofluvial deposits can be found in $[67,105]$.

\subsubsection{Earthquake related studies}

A class of sediments that is of relevance to the category of earthquake related luminescence dating studies are sandy deposits emplaced during a tsunami event. Tsunami events following an earthquake commonly transport sandy materials inland to deposit them in proximal tidal marshes as well as in bogs and lakes. The sand is subsequently overlain by other materials such as peat or mud to form part of the coastal depositional sequence. Dating such sands would allow one to reconstruct the recurrence rate of the tsunami events and, by inference the earthquakes themselves, which is pertinent information for evaluating environmental hazards within the coastal zone [29]. Tsunami events, however, occur abruptly and the turbidity associated with such an event would not normally provide adequate time for effective bleaching of the sands before they are deposited. This problem is avoided by targeting sands that were previously part of the tidal flat and tidal channel environment where they lay exposed at the surface prior to the tsunami [29]. Working on the west coast of North America, close to Washington and Vancouver Island, Huntley and Clague [108] were able to date two tsunami events using this approach. Other more recent studies that have attempted to date tsunami events include [109, 110]. It should be noted, however, that the identification of tsunami deposits within the coastal zone remains a subject of debate [for example, 111].

Another class of earthquake related sediments that have been dated using luminescence methods are deposits emplaced on horizontal surfaces that have been vertically displaced by 
faulting associated with earthquakes [29]. Contemporaneous deposition across the displaced surfaces will result in units of similar age on surfaces that differ in height, allowing the units to be correlated.

\section{Case study: Luminescence chronology of postglacial eolian dunes from Alberta, Canada: Constraining the timing of Late Pleistocene deglaciation}

To demonstrate the diverse aspects that can be encountered in a luminescence dating study, this section presents a brief outline of an investigation carried out in Alberta, western Canada [94] to constrain the timing of the retreat of the Laurentide Ice Sheet from the region. The landscape of Alberta features in excess of sixty discreet eolian dune fields that are believed to have been deposited after the retreat of the Laurentide Ice Sheet that once covered the most of Canada, east of the Rocky Mountains, about 20,000 years ago (Fig.10) [94]. Source sediments for the eolian deposits are thought to be sandy glaciolacustrine and outwash deposits associated with the retreating ice sheet. The eolian dunes in central and northern Alberta are currently stable, with many of them supporting boreal vegetation. It is thought that the eolian deposition was initiated in the immediate aftermath of the retreat of the Laurentide Ice Sheet but before the climate conditions ameliorated enough to allow vegetation to flourish. Once the climate improved, the landscape was stabilized by vegetation and the dunes have remained largely intact such that the depositional sequences they contain can be used as indicators of past environmental conditions. The exact chronology of the retreat of the Laurentide Ice Sheet from the region, however, still has to be firmly established [94, 112]. Efforts to constrain the ice sheet's retreat using radiocarbon chronology has been hampered by the scarcity of contemporaneous radiocarbon bearing material from the region $[94,113,114]$ which contrasts with areas further to the east $[112,113]$, to the west [115] or to the south [116; 117] where radiocarbon ages have provided a sound chronological framework for Late Pleistocene deglaciation. To help address this lack of age controls for the postglacial period, a study was conceived to collect eolian dune sands from central and northern Alberta and date them using luminescence methods [94]. Ages from the eolian dunes would have a number of contributions. Because the transport and deposition of eolian sediment can only take place in ice-free conditions, ages from the dune sands would make it possible to determine by when the Laurentide Ice Sheet had retreated from the landscape. It would also be possible to construct a chronological framework for the environmental evolution of the region by putting maximum age constrains on the colonisation of the region by vegetation. A third important aspect of establishing the chronology of deglaciation of the region was that it would allow investigators to evaluate whether western Canada served as an inland migration route used by the first humans to reach the Americas. This is because the path followed by the first Americans remains a subject of contention. Evidence from central USA shows that humans had settled there by about 15,000-16,000 years ago [118]. These humans are thought to have migrated south from Beringia, having arrived from Eurasia earlier during a low sea-level phase. Around 15,000-16,000 years ago, however, some researchers believe that the Laurentide Ice Sheet still covered large parts of western Canada, including Alberta, making the region unnavigable for 
humans [119]. Hence, an alternative route must have been used, possibly a coastal route [120]. By accurately constraining the timing of the retreat of the ice sheet using the chronology of dune deposition, however, it would be possible to ascertain if the ice sheets retreated early enough to allow humans to trek from Beringia through western Canada to reach central USA by around 15,000-16,000 years ago using an inland route.

Accordingly, samples for luminescence dating were collected from eolian dunes in central and northern Alberta from excavation pits as well from vertical profiles and these were sent to luminescence dating labs at the University of Washington and Utah State University for analysis [94]. At both labs the sample preparation methods followed standard procedures that included sieving, heavy liquid separation and etching with HF to separate quartz. The SAR protocol $[26,27]$ was used determine the paleodose and luminescence measurements were conducted on a Risø DA-20 instrument. For the dose rate, concentrations of U, Th and K were determined using ICP-MS and inductively coupled plasma atomic emission spectroscopy (ICP-AES). Alternatively TSAC in conjunction with flame photometry and beta counting were also used. More experimental details can be found in [94].

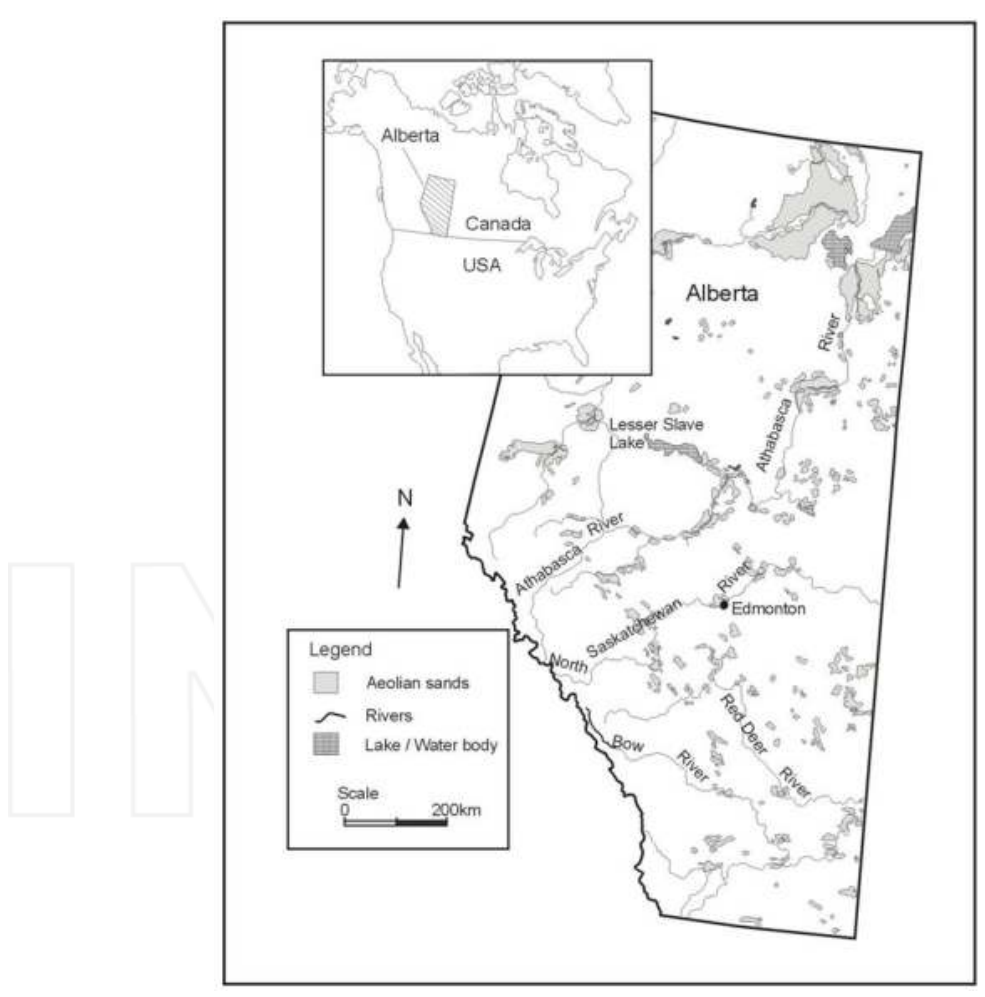

Figure 10. Eolian sands in Alberta, Canada. Samples were collected from dunes in central and northern Alberta from which quartz was extracted for dating using the SAR protocol [94]. 
Results of the study showed that eolian deposition in central Alberta started at least $15,000-16,000$ years ago and that by around 11,000-12,000 years ago, many of the dunes had become stable [94]. A separate investigation [93] that had dated feldspar separates from dune sands in the area using the additive dose protocol obtained similar ages. The age of $15,000-16,000$ years ago for eolian deposition in the study area is important because it shows that, by that time, the Laurentide Ice Sheet had retreated from the region, allowing eolian processes to operate. However, it is not yet possible to establish when exactly the eolian conditions began but, with the arrival of humans in central USA at around 15,000-16,000 ka, it is possible that the ice sheet retreated from western Canada early enough to avail the first Americans an inland route to trek southwards [94]. With regards to the subsequent colonisation of the region by boreal vegetation, the termination of eolian deposition around $11,00-12,000$ years ago is consistent with other records which point to the proliferation of vegetal communities in the region associated with the postglacial climate amelioration of the Early Holocene [94]. Continuing work in the study area focuses on establishing a higher resolution chronology of eolian deposition, with emphasis on obtaining a more accurate framework for the initiation of eolian processes in the region [94].

\section{Current and future trends in luminescence dating}

Over the last forty years, luminescence dating has matured into a full-fledged and robust technique with many practising laboratories established across the world. Every three years, international practitioners gather for the Luminescence and Electron Spin Resonance Dating (LED) Conference where research in luminescence dating is presented. In the intervening years between the LED conferences, national and regional meetings such as the UK Luminescence Dating Conference, the German Luminescence and ESR Conference, and the New World Luminescence Dating Workshop in North America are also held. Such meetings feature fundamental research into luminescence dating methods as well as their applications in the environmental, geological and archaeological sciences. Advances in instrumentation are also always an important component at the meetings.

Prominent topics in current fundamental research include efforts to better understand the luminescence signal characteristics of both quartz and feldspar, which should allow for more accurate ages to be produced as well as for the dating ranges to be extended. For instance, ongoing studies are trying to characterise the behaviour of quartz at high doses. As indicated earlier, a number of studies have shown that at high doses (>150Gy), quartz OSL SAR protocols produce ages that underestimate the real ages of dated sedimentary units [for example, 70, $102,121]$. As a result, ongoing studies aim to identify the causes of these underestimations, particularly by looking at the characteristics of the individual components of the luminescence signal (for example, fast, medium and slow). Success in this quest could lead to the development of appropriate protocols that would make it possible to extend the dating range of quartz beyond what is currently attainable.

For feldspar, researchers have been looking at identifying IRSL signals that are less susceptible to anomalous fading which would also allow much older ages to be determined [for example, 
$6,122,123,124]$. As indicated earlier, though quartz is usually favoured for dating because it does not exhibit fading problems like those observed in feldspar, its drawback is that it saturates at much lower doses ( $<150 \mathrm{~Gy})$. The advantage that feldspar has over quartz, however, is encumbered by the lack of fading correction methods that can be used on older ages ( $>100 \mathrm{~Gy}$ ) because fading correction methods proposed to date are normally only effective for low doses $[43,44]$. Hence, any methodology that would allow feldspar grains with high doses to yield accurate ages would be desirable. Accordingly, recent studies have shown that IRSL signals obtained by stimulating feldspars at a low temperature (for example, $50{ }^{\circ} \mathrm{C}$ ) immediately followed by another IRSL measurement at an elevated temperature (for example, $290{ }^{\circ} \mathrm{C}$ ) yields a signal that has a lower fading rate. This measurement protocol is referred to as post-IR IRSL [122] and results provided to date suggest this is a promising approach that has the potential to extend the dating range of feldspars significantly $[6,125]$.

Developments in instrumentation are also keeping pace, with new luminescence measurement systems being developed [for example, 126]. Also worth mentioning is the recent development of portable OSL devices capable of conducting rapid measurements in the field [for example, 127]. Though limited in capability compared to regular OSL readers, such portable devices could, with additions such as internal X-ray radiation sources, introduce more options in the sphere of luminescence signal collection.

Overall, the field of luminescence dating is a vibrant research area and, if the recent past is any indication of what the future holds, it is a discipline guaranteed to witness innovative developments in the coming years. With the continual refinement of both the laboratory procedures as well as the equipment, we should see chronologies being reported with greater precision and accuracy.

\section{Author details}

Ken Munyikwa*

Address all correspondence to: kenm@athabascau.ca

Centre For Science, Athabasca University, University Drive, Athabasca, Alberta, Canada

\section{References}

[1] Huntley DJ, Godfrey-Smith DI, Thewalt MLW. Optical dating of sediments. Nature 1985; 313: 105-107.

[2] Aitken MJ. Luminescence dating: a guide for non-specialists. Archaeometry 1989; 31: 147-159. 
[3] Wintle AG. Luminescence dating: where it has been and where it is going. Boreas 2008; 37: 471-482.

[4] Murray A, Buylaert J-P, Henriksen M, Svendsen J-I, Mangerud J. Testing the reliability of quartz OSL ages beyond the Eemian. Radiation Measurements 2008; 43: 776-780.

[5] Pawley SM, Bailey RM, Rose J, Moorlock BSP, Hamblin RJO, Booth SJ, Lee JR. Age limits on Middle Pleistocene glacial sediments from OSL dating, north Norfolk, UK. Quaternary Science Reviews 2008; 27: 1363-1377.

[6] Buylaert JP, Murray AS, Thomsen KJ, Jain M. Testing the potential of an elevated temperature IRSL signal from K-feldspar. Radiation Measurements 2009; 44: 560-565.

[7] Daniels F, Boyd CA, Saunders DF. Thermoluminescence as a research tool. Science 1953; 117: 343-349.

[8] Aitken MJ, Tite MS, Reid J. Thermoluminescence dating of ancient ceramics. Nature 1964; 202: 1032-1033.

[9] Kennedy GC, Knopff L. Dating by thermoluminescence. Archaeology1960; 13: 147-148.

[10] Grogler N, Houtermans F G, Staufer H. Uber die datierung con keramik und ziegeldurchthermolumineszenz. Helv.Phys. Acta.1960; 33: 595-6.

[11] Zeller EJ, Wray JL, Daniels F. Factors in age determination of carbonate sediments by Thermoluminescence. Bulletin of the American Association of Petroleum Geologists 1957; 41: 121-129.

[12] Sabels BE. Age studies on basaltic flows using natural alpha activity and thermoluminescence. Proceedings of the Symposium on Radioactive Dating, Athens. International Atomic Energy Agency, Vienna, 87, November 1962: Athens Greece.

[13] Morozov GV. The relative dating of Quaternary Ukranain sediments by the thermoluminescence method, unpublished paper presented at the 8th International Quaternary Association Congress, Paris,1968. In the Geol. Survey Library, Washington, D.C. cat no. 208M8280. 167.

[14] Shelkoplyas VN. Dating of the Quaternary deposits by means of thermoluminescence in chronology of the glacial age. Geographic Society of the U.S.S.R., Pleistocene Commission, Leningrad 1971; 155-160.

[15] Dreimanis A, Hutt G, Raukas A, Whippey PW. Dating methods of Pleistocene deposits: thermoluminescence. Geoscience Canada 1978; 5: 55-60.

[16] Wintle AG, Huntley DJ. Thermoluminescence dating of deep-sea sediment core. Nature 1979; 279: 710-12. 
[17] Wintle AG, Huntley DJ. Thermoluminescence dating of ocean sediments. Canadian Journal of Earth Science 1980; 17: 348-690.

[18] Huntley DJ. On the zeroing of the thermoluminescence of sediments. Physics and Chemistry of Minerals 1985; 12: 122-127.

[19] Godfrey-Smith DI, Huntley DJ, Chen W-H. Optical dating studies of quartz and feldspar sediment extracts. Quaternary Science Reviews 1988; 7: 373-380.

[20] Hütt G, Jack I, Tchonka J. Optical dating: K-feldspars optical response stimulation spectrum. Quaternary Science Reviews 1988; 7: 381-386.

[21] Duller GAT. Equivalent dose determination using single aliquots. Nuclear Tracks and Radiation Measurements 1991; 18: 371-378.

[22] Galloway RB. Equivalent dose determination using only one sample: Alternative analysis of data obtained from infrared stimulated luminescence of feldspars. Radiation Measurements 1996; 26: 103-106.

[23] Murray AS, Roberts RG, Wintle AG. Equivalent dose measurement using a single aliquot of quartz. Radiation measurements 1997; 27: 172-84.

[24] Bøtter-Jensen L, Duller GAT, Murray AS, Bannerjee D. Blue light emitting diodes for optical stimulation of quartz is retrospective dosimetry and dating. Radiation Protection Dosimetry 1999; 84: 335-40.

[25] Duller GAT, Bøtter-Jensen L, Kohsiek P, Murray AS. A high sensitivity optically stimulated luminescence scanning system for measurement of single sand-sized grains. Radiation Protection Dosimetry 1999; 84: 325-330.

[26] Murray AS, Wintle AG. Luminescence dating of quartz using an improved single-aliquot regenerative-dose protocol. Radiation Measurements 2000; 32: 57-73.

[27] Murray AS, Wintle AG. The single aliquot regenerative dose protocol: potential improvements in reliability. Radiation Measurements 2003; 37: 377-381.

[28] Wintle AG. Fifty years of luminescence dating. Archaeometry 2008; 50: 276-312.

[29] Aitken MJ. An Introduction to Optical Dating. Oxford: Oxford University Press; 1998

[30] Prescott JR, Hutton JT. Cosmic ray contributions to dose rates for luminescence and ESR dating; large depths and long-term time variations. Radiation Measurements 1994; 23: 497-500.

[31] Stokes S. Luminescence dating applications in geomorphological research. Geomorphology 1999; 59: 153-171.

[32] Aitken MJ. Thermoluminescence dating. London: Academic Press; 1985.

[33] Fleming SJ. Thermoluminescent dating: refinement of the quartz inclusion method. Archaeometry 1970; 12: 133-45. 
[34] Huntley DJ, Godfrey-Smith DI, Thewalt, MLW, Berger GW. Thermoluminescence spectra Arising from optical ejection of trapped charges in feldspars. Journal of Luminescence 1988; 44: 41-46.

[35] McKeever SWS. Thermoluminescence of Solids. Cambridge. Cambridge University Press; 1985.

[36] Wintle AG. Luminescence dating: laboratory procedures and protocols. Radiation Measurements 1997; 27: 769-817.

[37] Scholefield RB, Prescott JR, Franklin AD, Fox PJ. Observations on some thermoluminescence emission centres in geological quartz. Radiation Measurements 1994; 23: 409-412.

[38] Botter-Jensen L. Luminescence techniques: instrumentation and methods. Radiation Measurements 1997; 27: 749-768.

[39] Bailey RM, Smith BW, Rhodes EJ. Partial bleaching and the decay form characteristics of quartz OSL. Radiation Measurements 1997; 27: 124-136

[40] Jain M, Murray AS, Bøtter-Jensen L. Characterisation of blue-light stimulated luminescence components in different quartz samples: implications for dose measurement. Radiation Measurements 2003; 37: 441-449.

[41] Bulur E. An Alternative technique for optically stimulated luminescence (OSL) experiment. Radiation Measurements 1996; 26: 701-709.

[42] Wintle AG. Anomalous fading of thermoluminescence in mineral samples. Nature $1973 ; 245,143-144$.

[43] Huntley DJ, Lamothe M. Ubiquity of anomalous fading in feldspars and the measurements and correction of it in optical dating. Canadian Journal of Earth Sciences 2001; 38: 1093-1106.

[44] Lamothe M, Auclair M. A solution to anomalous fading and age shortfalls in optical dating of feldspar minerals. Earth and Planetary Science Letters 1999; 171: 319-323.

[45] Duller GAT. Behavioural studies of stimulated luminescence from feldspars. Radiation Measurements 1997; 27: 663-694.

[46] Huntley DJ, Godfrey-Smith DI, Thewalt MLW, Berger, GW. Thermoluminescence spectra of some mineral samples relevant to thermoluminescence dating. Journal of Luminescence 1988; 39: 123-136.

[47] Krbetschek MR, Gotze J, Dietrch A, Trautmann T. Spectral information from minerals relevant for luminescence dating. Radiation Measurements 1997; 27: 695-748.

[48] Jungner H, Huntley DJ. Emission spectra of some potassium feldspars under $633 \mathrm{~nm}$ stimulation. Nuclear Tracks and Radiation Measurements 1991; 18: 125-126. 
[49] Li S-H, Tso M-Y W. Lifetime determination of OSL signals from potassium feldspar. Radiation Measurements 1997; 27: 119-121.

[50] Bøtter-Jensen L, Duller GAT, Poolton NRJ. Excitation and emission spectrometry of stimulated luminescence from quartz and feldspars. Radiation Measurements 1994; 23: 613-616.

[51] Barnett SM, Bailiff IK. Infrared stimulation spectra of sediment containing feldspars. Radiation Measurements 1997; 27: 237-242.

[52] Huntley DJ, Godfrey-Smith DI, Haskell EH. Light-induced emission spectra from some quartz and feldspars. Nuclear Tracks and Radiation Measurements 1991; 18: 127-131.

[53] Krbetschek MR, Rieser U, Stloz W. Optical dating: some dosimetric properties of natural feldspars. Radiation Measurements 1996; 27: 107-117.

[54] Ugumori T, Ikeya M. Luminescence of CaCO3 under N2 laser excitation and application to archaeological dating. Japanese Journal of Applied Physics 1980; 19: 459-465.

[55] Templer RH, Smith BW. Auto-regenerative TL dating with zircon from fired materials. Nuclear Tracks and Radiation Measurements 1988; 14: 329-332.

[56] Smith BW. Zircons from sediments: a combined OSL and TL auto-regenerative dating technique. Quaternary Science Reviews 1988; 7: 401-406.

[57] Pietsch TJ, Olley JM, Nanson GC. Fluvial transport as a natural luminescence sensitizer of quartz. Quaternary Geochronology 2008; 3: 365-376.

[58] Murray AS, Roberts RG. Measurement of the Equivalent dose in quartz using a regenerative-dose single aliquot protocol. Radiation Measurements 1998; 29: 503-515.

[59] Stokes S. The timing of OSL sensitivity changes in a natural quartz. Radiation Measurements 1994; 23: 601-605.

[60] Mejdhal V, Botter-Jensen L. Luminescence dating of archaeological materials using a new technique based on single aliquot measurements. Quaternary Science Reviews (Quaternary Geochronology) 1994; 13: 551-554.

[61] Murray AS. Developments in optically stimulated luminescence and photo-transferred thermoluminescence dating of young sediments: application to a 2000-year sequence of flood deposits. Geochimica et Cosmochimica Acta 1996; 60: 565-576.

[62] Wintle AG, Murray AS. A review of quartz optically stimulated luminescence characteristics and their relevance in single-aliquot regeneration dating protocols. Radiation Measurement 2006; 41: 369-391.

[63] Olley JM, Catcheon GG, Roberts RG. The origin of dose distribution in fluvial sediments, and the prospect of dating single grains from fluvial deposits using optically stimulated luminescence. Radiation Measurements 1999; 30: 207-217. 
[64] Lian OB, Roberts RG. Dating the Quaternary: progress in luminescence dating of sediments. Quaternary Science Reviews 2006; 25: 2449-2468.

[65] Galbraith RF, Roberts RG, Laslett GM, Yoshide H, Olley JM. Optical dating of single and multiple grains of quartz from Jinmium Rock Shelter, Northern Australia: Part 1, Experimental design and statistical models. Archaeometry 1999; 41: 339-364.

[66] Olley JM, Roberts RG, Yoshida H, Bowler JM. Single-grain optical dating of grave infill associated with human burials at Lake Mungo, Australia. Quaternary Science Reviews 2006; 25: 2469-74.

[67] Fuchs M, Owen LA. Luminescence dating of glacial and associated sediment: review, recommendations and future directions. Boreas 2008; 31: 636-659.

[68] Ballarini M, Wallinga J, Murray AS, van Heteren, S, Oost AP, Bos, van Ejk, CWE. Optical dating of young coastal dunes on a decadal time scale. Quaternary Science Reviews 2003; 22: 1011-1017.

[69] Duller GAT. Luminescence dating of Quaternary sediments: recent advances. Journal of Quaternary Science 2004; 19: 183-192.

[70] Murray AS, Funder S. Optically stimulated luminescence dating of Danish coastal marine deposit: a test of accuracy. Quaternary Science Reviews 2003; 22: 1177-1183.

[71] Buylaert. JP, Murray AS, Huot S. Optical dating of an Eemian site in northern Russia using K-feldspar. Radiation Measurements 2008; 43: 505-512.

[72] Stevens T, Armitage SJ, Lu H, Thomas DSG. Examining the potential of high-resolution OSL dating of Chinese loess. Quaternary Geochronology 2007; 2: 15-22.

[73] Munyikwa K, Telfer M, Baker I, Knight C. Core drilling of Quaternary sediments for luminescence dating using the Dormer DrillmiteTM. Ancient TL 2011; 29: 15-22.

[74] Stokes S. Optical dating of young sediments using quartz. Quaternary Science Reviews (Quaternary Geochronology) 1992; 11: 153-159.

[75] Huntley DJ, Hutton JT, Prescott JR. Further thermoluminescence dates from the dune sequence in the south-east of South Australia. Quaternary Science Reviews 1994; 13: 201-207.

[76] Rees-Jones J. Optical dating of young sediments using fine grain quartz. Ancient TL 1995; 13: 9-14.

[77] Lian OB, Hu J, Huntley DJ, Hicock, SR. Optical dating studies of Quaternary organicrich sediments from southwestern British Columbia and northwestern Washington State. Canadian Journal of Earth Science 1995; 32: 1194-1207.

[78] Vandiver PB, Soffer O, Klima B, Svoboda J. The origins of ceramic technology at Dolni Vestonice, Czechoslovakia. Science 1989; 246: 1002-1008. 
[79] Mejdahl V. Feldspar inclusion dating of ceramics and burnt stones. PACT 1983; 9: 351-364.

[80] Huxtable J, Aitken MJ, Bonhommet N. Thermoluminescence dating of sediment baked by lava flows of the Chaine des Puys. Nature 1978; 275: 207-209.

[81] Berger GW, Huntley DJ. Dating volcanic ash by thermoluminescence. PACT 1983; 9: 581-592.

[82] Guerin G, Valladas G. Thermoluminescence dating of volcanic plagioclases. Nature 1980; 286: 697-699.

[83] Sutton SR. TL measurements on shock-metamorphosed sandstone and dolomite from Meteor Crater, Arizona: Pt. 1 shock dependence of TL properties. Journal of Geophysical Research 1985; 9: 3683-3689.

[84] Singhvi AK, Porat N. Impact of luminescence dating on geomorphological and palaeoclimate research in drylands. Boreas 2008; 37: 536-558.

[85] Pye K, Tsoar H. Aeolian Sand and Sand dunes. Heidelberg: Springer; 2009.

[86] Stokes S, Gaylord, DR. Optical dating of Holocene dune sands in the Ferris dune field, Wyoming. Quaternary Research 1993; 39: 274-281.

[87] Nanson GC, Price DM, Short SA. Wetting and drying of Australia over the last 300. Geology 1992; 20: 791-794.

[88] Munyikwa K. Synchrony of southern hemisphere late Pleistocene arid episodes: a review of luminescence chronologies from arid aeolian landscapes south of the Equator. Quaternary Science Reviews 2005; 24: 2555-2583.

[89] Telfer MW, Thomas DSG. Late Quaternary linear dune accumulation and chronostratigraphy of the southwestern Kalahari: implications for aeolian palaeoclimatic reconstructions and predictions of future dynamics. Quaternary Science Reviews 2007; 26: $2617-2630$.

[90] Hülle D, Hilgers A, Radtke U, Stolz C, Hempelmann N, Grunert J, Felauer T, Lehmkuhl F. Quaternary Geochronology 2010; 5: 107-113.

[91] Forman SL, Oglesby R, Webb RS. Temporal and spatial patterns of Holocene dune activity on the Great Plains of North America: Megadroughts and Climate Links: Global and Planetary Change, 2001; 29: 1-29.

[92] Goble RJ, Mason JA, Loope DB, Swinehart JB. Optical and radiocarbon ages of stacked palaeosols and dune sands in the Nebraska Sand Hills, USA. Quaternary Science Reviews 2004; 23: 1173-1182.

[93] Wolfe SA, Huntley DJ, Ollerhead J. Relict Late Wisconsinan dune fields of the Northern Great Plains. Canada. Géographie physique et Quaternaire 2004; 58: 323-336. 
[94] Munyikwa K, Feathers J, Rittenour T, Shrimpton H. Constraining the Late Wisconsinan retreat of the Laurentide Ice Sheet from western Canada using luminescence ages from postglacial eolian dunes. Quaternary Geochronology 2011; 6: 407-422.

[95] Frechen M, Vanneste K, Verbeeck K, Paulissen E, Camelbeeck T. The depositional history of the coversands along the Bree fault escarpment, NE Belgium. Geologie en Mijnbouw 2001; 80: 171-185.

[96] Tatumi SH, Yee M, Kowata EA, Carneiro A, Schwartz D. TL and OSL dating of the Negro River Basin, Brazil. Advances in ESR Applications 2002; 18: 187-191.

[97] Sommerville AA, Hanson JD, Housely RA, Sanderson DCW. Optically stimulated luminescence dating of coastal Aeolian sand accumulation in Sanday, Orkney Islands, Scotland. The Holocene 2007; 17: 627-637.

[98] Munyikwa K, Choi JH, Choi KH, Byun JM, Kim JW, Park K.. Coastal dune luminescence chronologies indicating a mid-Holocene highstand along the east coast of the Yellow Sea. Journal of Coastal Research 2008; 24: 92-103.

[99] Frechen M. Luminescence dating of loessic sediments from the Loess Plateau, China.Geologische Rundschau 1999; 87: 675-684.

[100] Watanuki T, Murray AS, Tsukamoto S. A comparison of OSL ages derived from silt sized quartz and polymineral grains from Chinese Loess. Quaternary Science Reviews 2003; 22: 991-997.

[101] Forman SL, Pierson J. Late Pleistocence luminescence chronology of loess deposition in the Missouri and Mississippi River valleys, United States. Palaeogeography, Paleoclimatology, Palaeoecology 2002; 186: 25-46.

[102] Lai Z, Fan A. Examining quartz OSL age underestimation for loess samples from Luochuan in the Chinese loess plateau. Geochronometria 2013; 41: 57-64.

[103] Rittenour TM. Luminescence dating of fluvial deposits: applications to geomorphic, palaeoseismic and archaeological research. Boreas 2008; 37: 613-635.

[104] Cunningham AC, Wallinga J. Realizing the potential of fluvial archives using robust OSL chronologies. Quaternary Geochronology, 2012; 12: 98-106.

[105] Thrasher IM, Mauz B, Chiverrell RC, Lang A. Luminescence dating of glaciofluvial deposits: a review. Earth Science Reviews 2009; 97: 133-146.

[106] Rhodes EJ, Bailey RM. The effect of thermal transfer on the zeroing of the luminescence of quartz from recent glaciofluvial sediments. Quaternary Geochronology 1997; 16: 291-298.

[107] Munyikwa K, Brown S, Kitabwala Z. Delineating stratigraphic breaks at the bases of postglacial dunes in central Alberta, Canada using a portable OSL reader. Earth Surface Processes and Landforms 2012; 37: 1603-1614. 
[108] Huntley DJ, Calgue JJ. Optical dating of tsunami-laid sands. Quaternary Research 1996; 46: 127-140.

[109] Lopez GI. Evidence for mid-to late-Holocene palaeo-tsunami deposits, Kakawis Lake, Vancouver Island, British Columbia. Natural Hazards 2011; 60: 43-68.

[110] Rebêlo L, Costas S, Brito P, Ferraz M, Prudencio I, Burbidge C. Imprints of the 1755 tsumani in the Troia Peninsula shoreline, Portugal. Journal of Coastal Research Special Issue 2013; 65: 814-819.

[111] Ramirez-Herrera MT, Lagos M, Hutchinson I, Kostoglodov V, Machain L, Caballero M, Goguitchaichvili A, Aguilar B, Chague-Goff C, Goff J, Ruiz-Fernandez AC, Ortiz M, Nava H, Bautista F, Lopez GI, Quintana P. Extreme wave deposits on the Pacific coast of Mexico: Tsunamis or storms- a multiproxy approach. Geomorphology 2012; 139-140: 360-371.

[112] Dyke AS, Andrews JT, Clark PU, England JH, Miller GH, Shaw J, Veillette JJ. The Laurentide and Inuitian Ice Sheets during the last glacial maximum. Quaternary Science Reviews 2002; 21: 9-31.

[113] Dyke AS, Andrews JT, Clark PU, England JH, Miller GH, Shaw J, Veillette JJ. Radiocarbon Dates pertinent to defining the Last Glacial Maximum for the Laurentide and Inuitian Ice Sheets. Open File 412. Ottawa: Geological Survey of Canada; 2012.

[114] Fisher TG, Waterson N, Lowell TV, Hadjas I. Deglaciation ages and meltwater routing in the Fort McMurray region. Northeastern Alberta and northwestern Saskatchewan, Canada. Quaternary Science Reviews 2009; 28: 1608-1624.

[115] Cosma TN, Hendy IL, Chang AS. Chronological constraints on Cordilleran Ice Sheet glaciomarine sedimentation from core MD02-2496 off Vancouver Island (western Canada). Quaternary Science Reviews 2008; 27: 941-955.

[116] Easterbrook DJ. Advance and retreat of Cordilleran Ice Sheets in Washington, USA. Géographie physique et Quaternaire 1992; 46(1): 51-68.

[117] Kovanen DJ, Easterbrook DJ. Timing and extent of Allerod and Younger Dryas age (ca. 12,500-10,000 14C yr B.P.) oscillations of the Cordilleran Ice sheet in the Fraser Lowland, Western North America. Quaternary Research 2002; 57: 208-224.

[118] Goebel T, Waters MR, O'Rourke DH. The late Pleistocene dispersal of modern humans in the Americas. Science 2008; 319: 1497-1501.

[119] Jackson Jr LE, Wilson MC. The ice-free corridor revisited. Geotimes 2004; 49: 16-29.

[120] Heatherington R, Barrie VJ, MacLeod R, Wilson M. Quest for lost land. Geotimes 2004; 49: 20-23.

[121] Murray AS, Svendsen JI, Mangerud J, Astakhov VI. Testing the accuracy of quartz OSL dating using known-age Eemian site on the river Sula, Northern Russia. Quaternary Geochronology 2007; 2: 102-109. 
[122] Thomsen KJ, Murray AS, Jain M, Bøtter-Jensen L. Laboratory fading rates of various luminescence signals from feldspar-rich sediment extracts. Radiation Measurements 2008; 49: 1474-1486.

[123] Thomsen KJ, Murrary AS, Jain M. Stability of IRSL signals from sedimentary K-feldspar samples. Geochronometria 2011; 38: 1-13.

[124] Buylaert JP, Thiel C, Murray AS, Vandenberghe DAG, Yi S, Lu H. IRSL and Post-IR IRSL residual doses recorded in modern dust samples from the Chinese loess plateau. Geochronometria 2011; 38: 432-440.

[125] Thiel C, Buylaert JP, Murray A, Terhorst B, Hofer I, Tsukamoto S, Frechen M. Luminescence dating of the Stratzing loess profile (Austria) - Testing the potential of an elevated temperature post-IR IRSL protocol. Quaternary International 2011; 234: 23-31.

[126] Richter D, Richter A, Dornich K. Lexsyg- A new system for luminescence research. Geochronometria 2011; 20(4): 220-228.

[127] Sanderson DCW, Murphy S. Using simple portable measurements and laboratory characterisation to help understand complex and heterogeneous sediment sequences for luminescence dating. Quaternary Geochronology 2010; 5: 299-305.
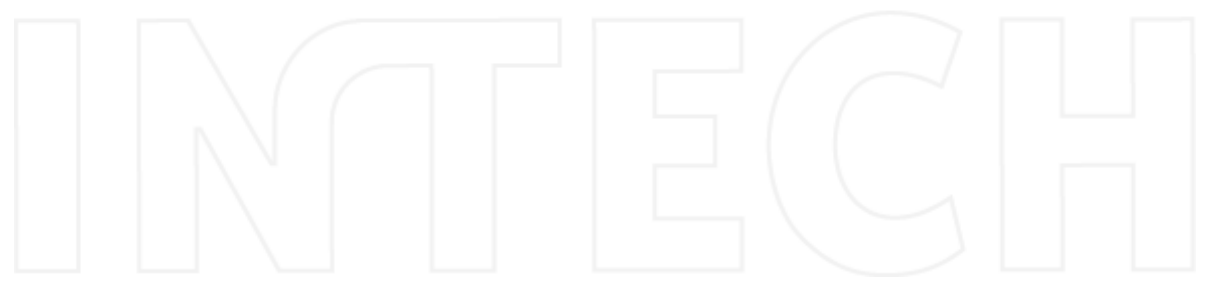

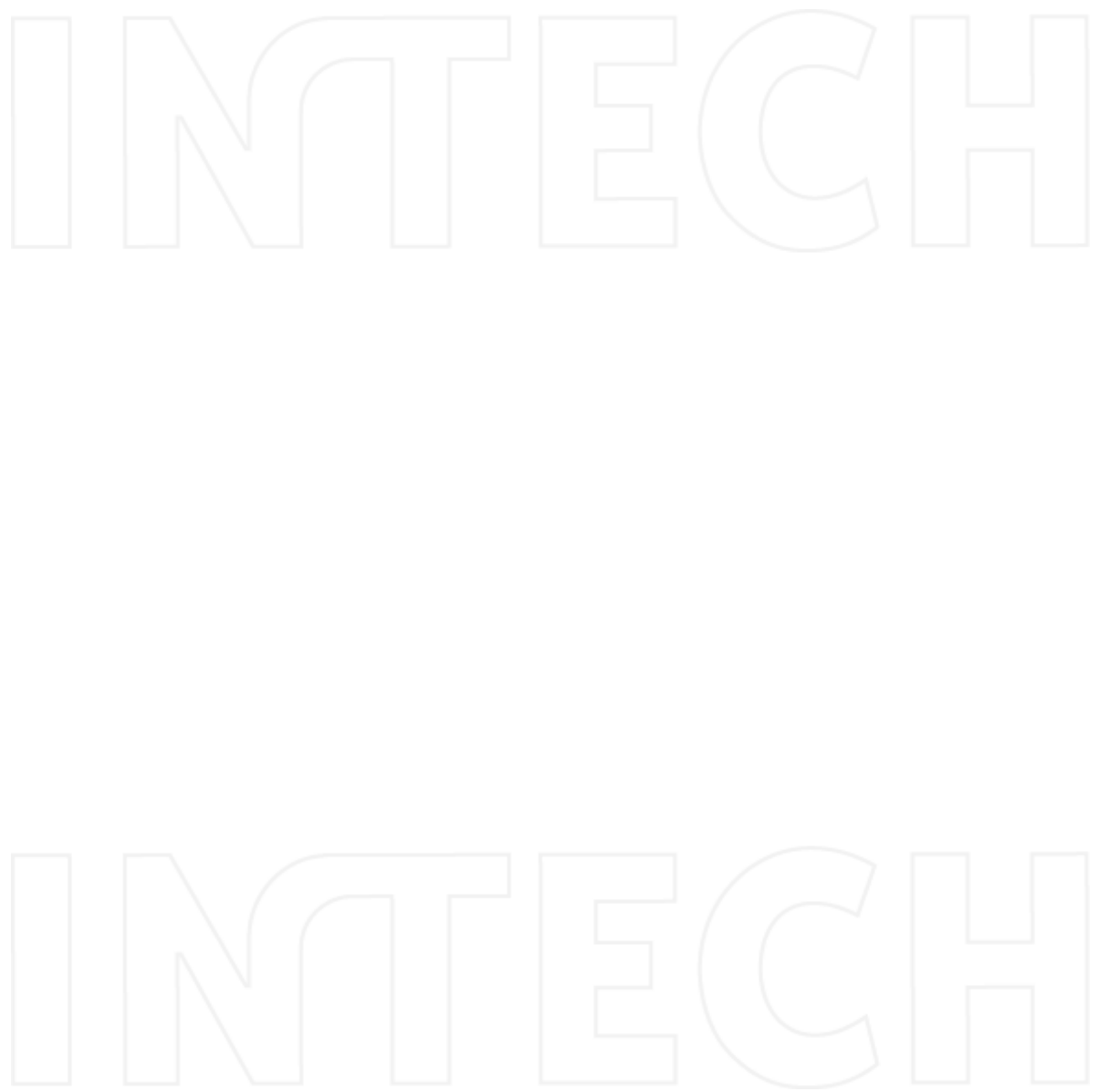


\title{
Chapter 3
}

\section{Varve Chronology}

\author{
Nils-Axel Mörner
}

Additional information is available at the end of the chapter

http://dx.doi.org/10.5772/58630
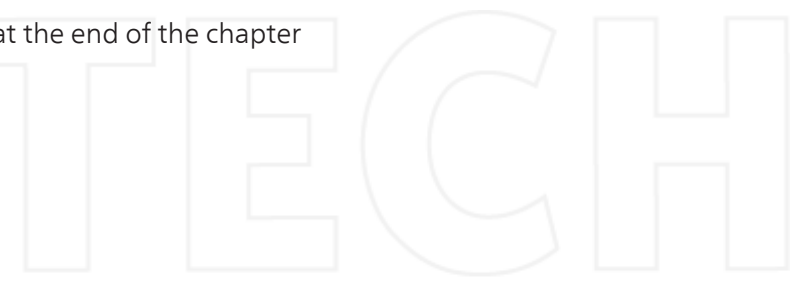

\section{Introduction}

Chronology indicates a sequence of time and refers back to Chronos, the Greek God of time. The word "varve" needs an explanation, however (varv is a Swedish word denoting a full circle). It refers to a rhythmic sequence representing the deposition of sediments or growth of a precipitate over a time of 1 single year (as defined by De Geer, 1884, Högbom, 1889 and Johnston, 1922). Consequently, a varve is a sedimentological equivalent to the biological growth rings in a tree known as tree-rings.

Like tree-rings, the varves are measured as to thickness. The variations in thick-ness over a varve sequence are then used to establish correlations with another, nearby sequences (Fig. 1). By extending these sequences piece by piece over time, we establish a varve chronology.

This method was invented in the late $19^{\text {th }}$ century by Gerard De Geer in Sweden (Fig. 1; further described in De Geer, 1940; Mörner, 1978; Francus et al., 2013). Therefore, it was often termed "the Swedish Varve Chronology" or "the Swedish Time Scale". Today, this chronology spans about 14,000 years from the present back in time. The method has been successfully applied in Finland, and also applied in many other areas of the globe (e.g. North America, the Alps, and Argentina).

Some sedimentary basins contain varved sediments where the individual varves may be counted separately (or at least approximated) so that site-specific long-term chronologies are established.

The present paper will be devoted to the Swedish Time Scale and the application of varve chronologies in general for precise dating of events, and calculations of rates. 


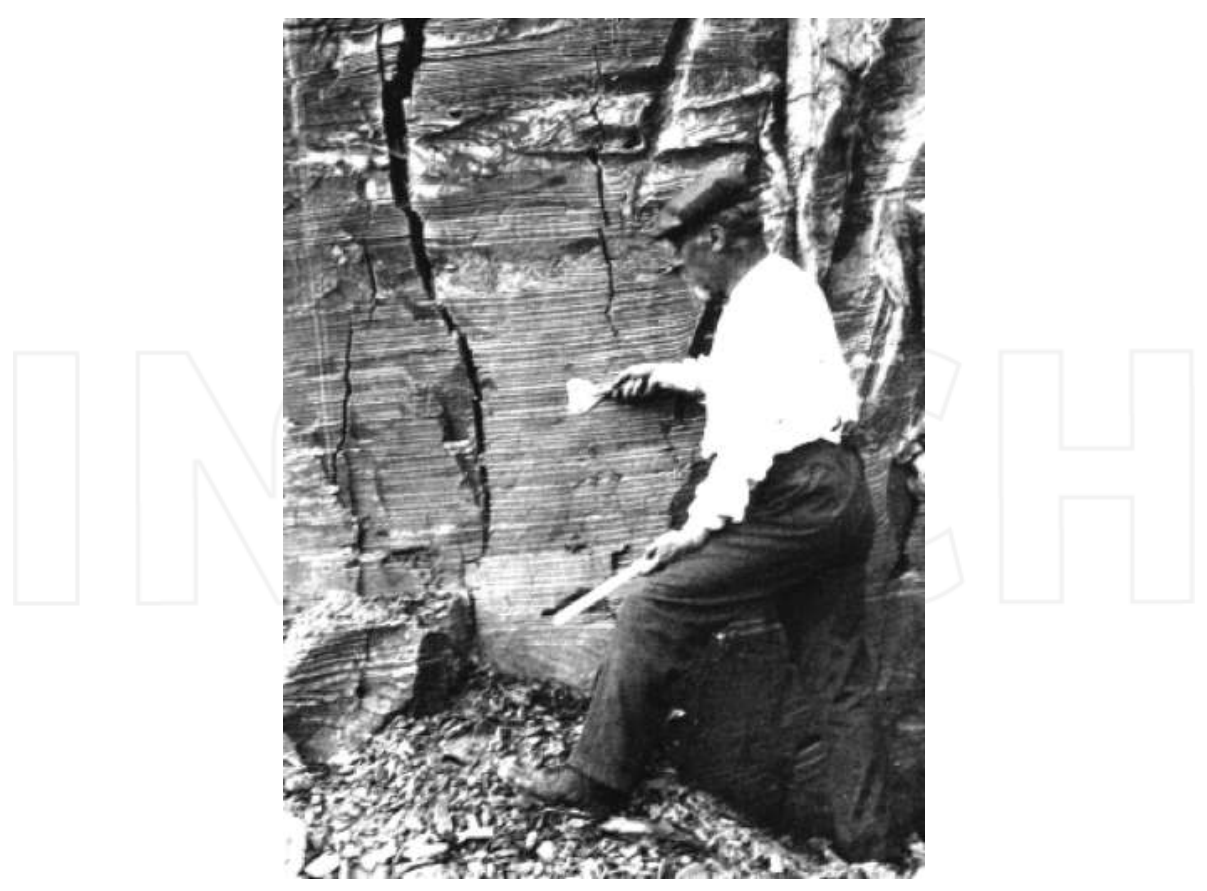

Figure 1. Gerard De Geer demonstrating the varve chronological method in Essex Junction, US, in 1920 (where he made the first measurements already in 1891).

\section{Building up the Swedish varve chronology}

In 1884, the Swedish geologist Gerard De Geer observed in a channel excavation in Stockholm that the basal clay was laminated in a fashion, which made him think in terms of the annual growth rings in trees. He noted that the lamina consisted of a lower unit that was lighter in colour and courser in grain size and an upper clay unit that was quite dark. He named those couplets "varves" and claimed that they represented annual deposition (De Geer, 1884, 1940). In 1889, Högbom showed that the ratio of magnesium carbonate and calcium carbonate differed in the two units, and he interpreted this in terms the annual geochemical changes in the Baltic.

In 1904, a period of intensive construction of new houses stated in Stockholm. This gave rise to excellent exposures of the sediment beds, including the basal "varved clay" (as it was now called). De Geer measured new exposures, and to his surprise he noted that the new diagrams correlated well not only in between themselves, but also with the diagram he had measured 20 years before, located some $3 \mathrm{~km}$ to the east. This convinced him partly that the varves really were true annual varves, and partly that he would now be able to build up a continual chronology. 
With intensive work by De Geer himself and his students, a chronology was built up from Stockholm to central Sweden. By this he could demonstrate that it took 1073 years for the land-ice to retreat from Stockholm to Jämtland in central Sweden, a distance of $500 \mathrm{~km}$. Already at the 1910 International Geological Meeting in Norden, he was able to give a detailed picture of the mode of ice recession after the last glaciation maximum around 20,000 years ago (De Geer, 1912).

\subsection{Identifying and measuring varves}

Because De Geer's primary aim was to date the recession of land-ice over Sweden, his and his students' work was concentrated on the oldest varve deposited in front of the receding ice margin. During the time of deglaciation, the crust was isostatically depressed by the load of the ice, causing relative sea level to be significantly higher than today. From the subglacial drainage system glacifluvial material was deposited in the form of eskers and varved clay with one varve for each year (as illustrated in Fig. 2).

The first varves to be deposited in front of the receding ice margin are strongly influenced by the glacial melting giving rise to varves composed of a coarse-grained summer unit (sandysilty, sometimes even gravelly) and a fine-grained winter unit (clay to fine silt). In an esker environment, the first summer unit may include many meters of gravelly sediments (e.g. Bergström, 1968). De Geer (1940) interpreted the sand units of the proximal varves as being "flocculated" through the water column in front of the ice. Kuenen (1951) showed, however, that these units must have been deposited as turbidites (bed-load transport). The thick sandy summer units of proximal varves often exhibit rhythmic laminations. Ringberg (1984) counted some 50 laminae and proposed that they represented the number of summer days with open water conditions in the Baltic.

The clayey winter units represent the slow setting of suspended matter during the winters. These beds are often dark to black, exhibiting a reducing environment. During the winters, the lake and sea levels froze over, turbulence ceased and calm water conditions were established allowing suspended matter to settle.

The annual rhythmicity behind De Geer's glacial varves was the annual changes between melting in the summers and freezing in the winters. When the ice was gone some 9000 years ago, climatic conditions like to day were established. Even then, however, "postglacial" varved sedimentary sequences were formed in some lakes (Renberg, 1983) and especially in the deposits of the main rivers in the north due to the annual rhythm of a strong spring melting and low water discharge in late summers and winters (Lidén, 1913, 1938).

Besides the strict building up of "the Swedish Time Scale" via multiple short-distance varve correlations from Stockholm to north central Sweden, De Geer (1940) also attempted so-called "telecorrelations" over inter-continental and even inter-hemispheral distances. It seems to be an unfortunate mistake, however (not further discussed in this paper). 


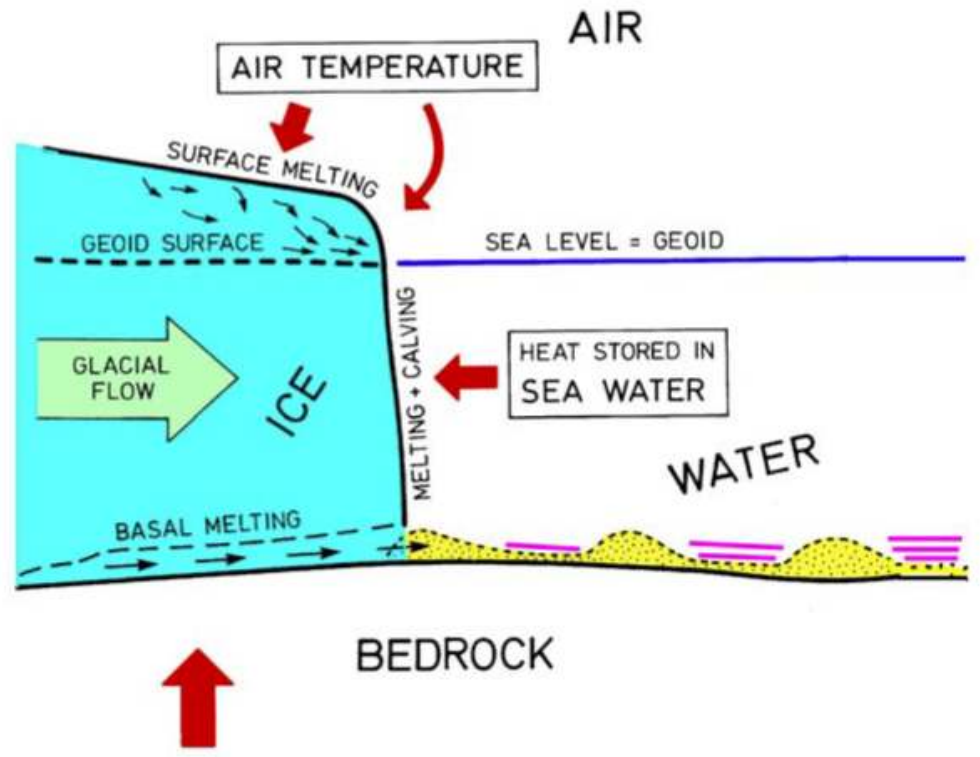

\section{HEAT FLOW}

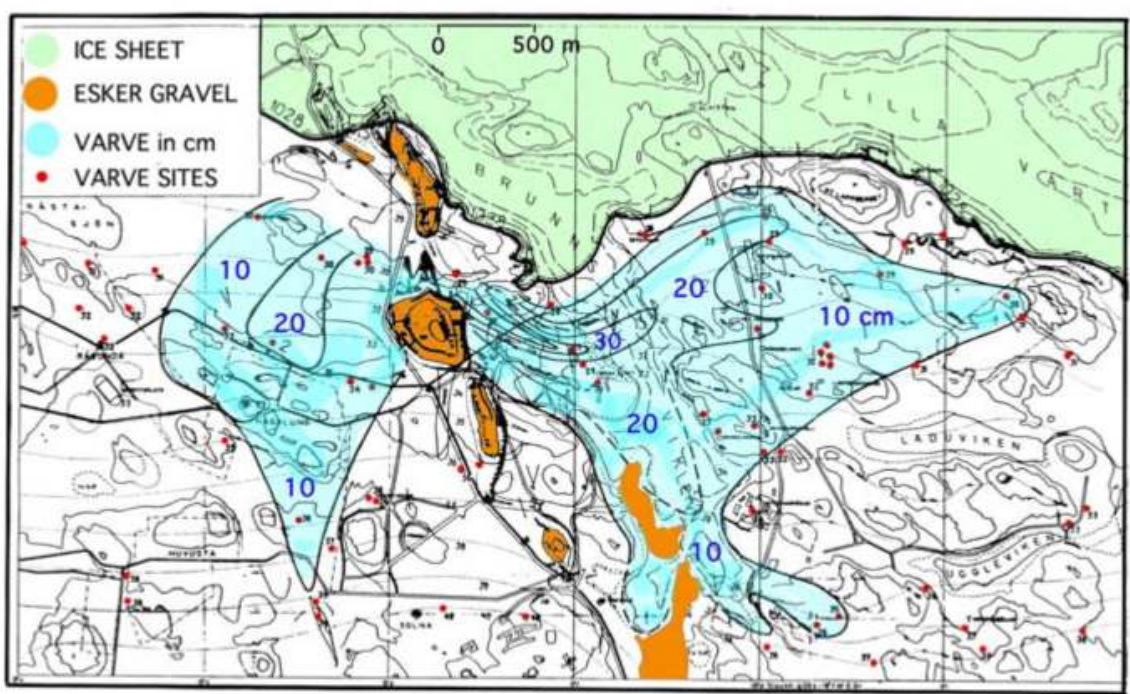

Figure 2. Above: The mode of ice melting, subglacial drainage and esker formation with 3 esker centra (yellow) and 3 successive annual varves (pink). Below: De Geer's (1940) map of the ice marginal position in year 10,385 BP, variations in thickness of this year's varve (blue), esker accumulations (orange) and varve sites (red dots). From Mörner (2008). 


\subsubsection{From field observations to chronological tools}

The varves are observed and recorded in open pits or in cores. An open pit is always better because it allow us to view the lateral variations. In cores, very long and continual sequences can be obtained, however. The "Swedish Foil Piston Corer" was designed just for this purpose allowing the retrieval of undisturbed cores of 11 m length (e.g. Järnefors, 1963).

De Geer introduced the simple method of rolling out a paper stripe over the section or core of varves and marking each individual varve on the stripe. Then the individual varve thicknesses were measured and plotted on a diagram. The saw-tooth patterns of the varve diagrams were then used for inter-site correlations.

Varves of special characteristics, "marker-varves", were sometimes used for correlations (e.g. De Geer, 1940; Bergström, 1968; Strömberg, 1989). Mörner (e.g. 2013) did the opposite, used the varve chronology to prove that a "marker varve" represented one single event and had a very wide lateral distribution.

Fig. 3 shows two cores taken close to each other in two successive years. Even visually, it is easy to see the nearly identical variations in varve thickness. The varve diagram shows variations that allow the correlation with the main Swedish Time Scale, so that absolute ages are obtained. In this case, traces of two separate earthquakes were identified and dated (Mörner, 2003, 2013a).

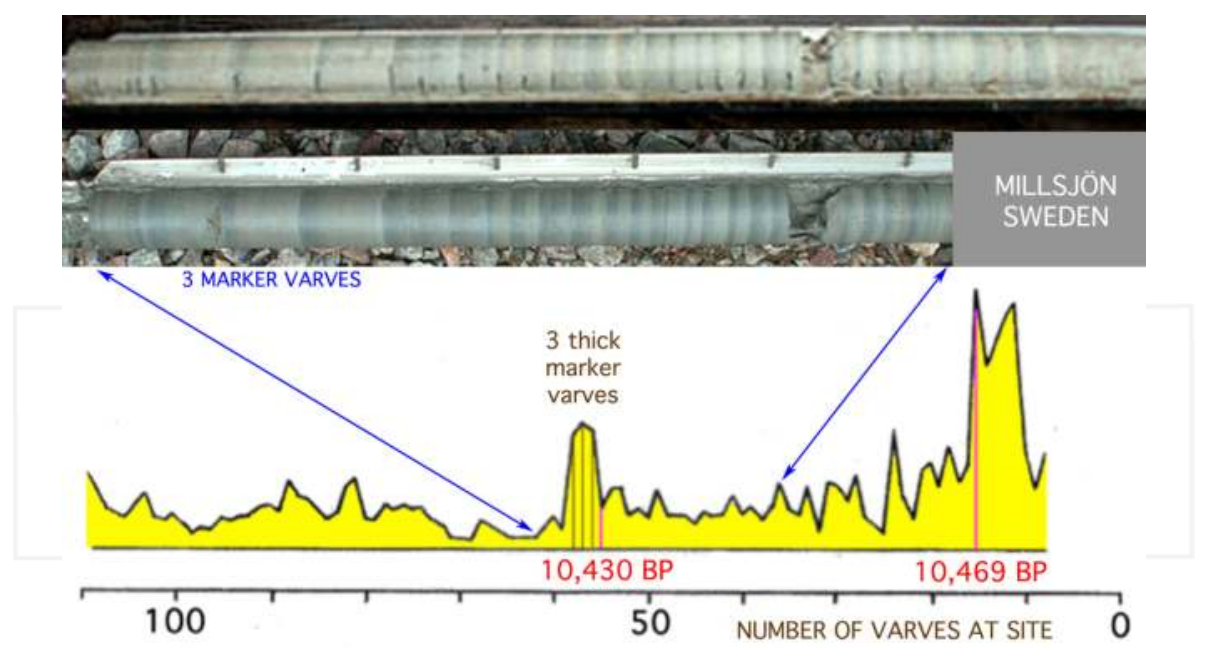

Figure 3. Varved clay cores take two years apart at approximately the same site. The inter-core correlations are very clear. The entire section includes 110 varves. Via the marker varves, the section can be correlated to the Swedish Time Scale and dated in absolute varve ages BP. 


\subsection{Additional applications}

Lidén (1938) measured 7522 postglacial varves in the fluvial deposits occurring along the River Ångermanälven (plus a gap of 980 years to year 1900). By this, the varve chronology was fixed to the present and we were able to talk in terms of absolute years. De Geer's (1940) varve-1073 referring to the onset of the marine Yoldia Sea stage in the Baltic was now dated in absolute years at $9625 \mathrm{BP}$ (later to be revised to 10,430 varves BP as discussed below).

Fromm (1938) measured pollen and diatoms in the same varves, implying that we from that time on were able to know the absolute ages of the immigration of different tree species, and the changes between fresh-water and marine stages of the Baltic.

Because Lidén's work referred to the succession of river deltas, he achieved a curve of the relative land uplift dated in absolute years BP (Fig. 4). It became a fundamental tool for the understanding of the concept of glacial isostsy (Gutenberg, 1941; Mörner, 1979).

All this was, of course, quite remarkable at a time period were we generally lacked other means of establishing absolute time.

The varve chronology flourished also in Finland (Sauramo, 1923), and was also applied to eastern North America by Antevs (e.g. 1932) and Patagonia (Caldenius, 1932).

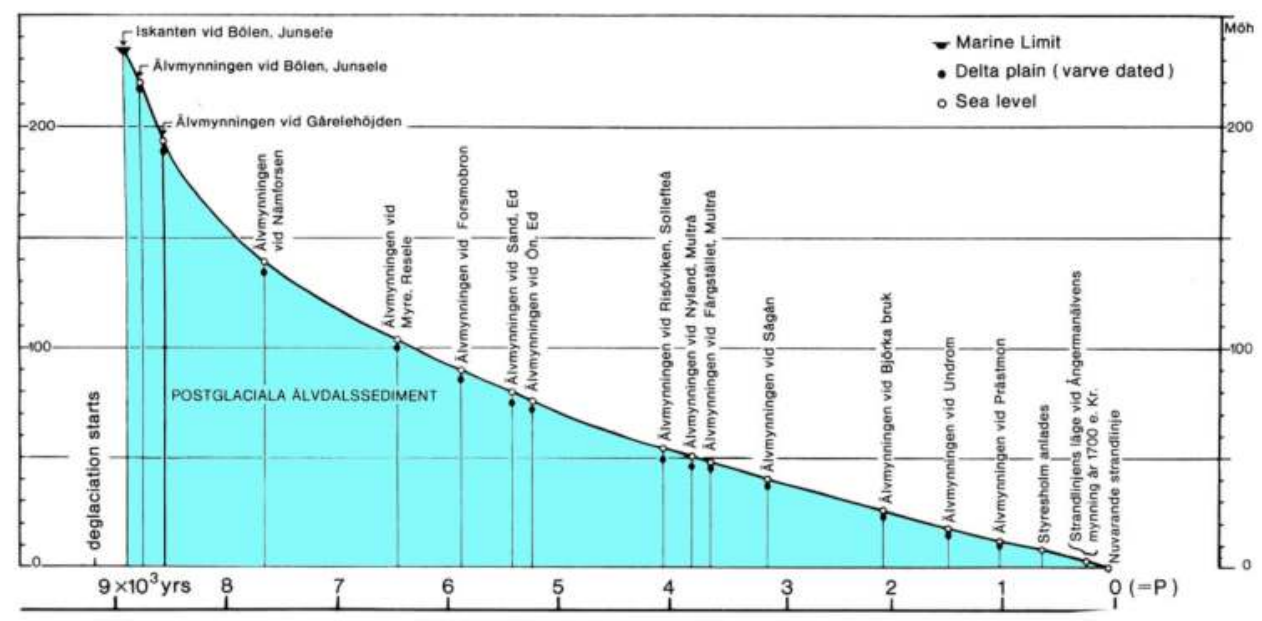

Figure 4. Varve-dated shorelevel displacement curve from Ångermanland by Lidén (1938; as redrawn in Mörner, 1979).

\section{A period of hesitation and change of focus}

With the introduction of the radiocarbon dating method (Arnold and Libby, 1949) things changed, and there suddenly was an alternative method of obtaining absolute ages. Also, quite 


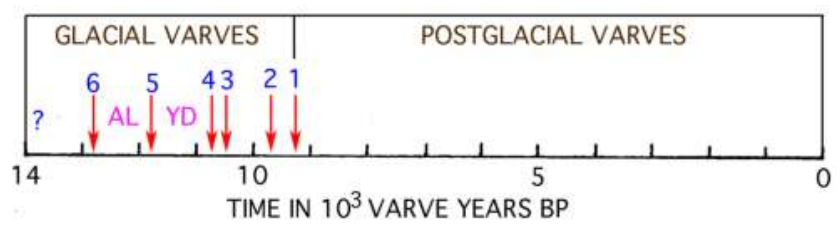

Figure 5. The Swedish Varve Chronology (or Swedish Time Scale) covers about 14,000 varve years back in time: (1) the drainage of the Central Jämtland Ice Lake at varve 9239 BP, (2) a major >8 earthquake at Hudiksvall at varve 9663 BP (and 9150 C14-years BP), (3) a major $>8$ earthquake with ingression of salt water into the Baltic basin and the onset of the Yoldia Sea stage (sensu strictu) at 10,430 BP, (4) the Drainage of the Baltic Ice Lake, roughly corresponding to the end of the Younger Dryas Stadial, at 10,740 BP, (5) the onset of the Younger Dryas (YD) cold period, (6) the onset of the Alleröd warm period.

bad errors in the varve ages were documented; especially in eastern US and Canada (e.g. Ridge and Larsen, 1990).

Internationally, the application of varve dating, rather switched from the ice recessional records in Sweden (De Geer, 1940), Finland (Sauramo, 1923) and North America (Antevs, 1932) to chronologies of continual lake records.

Annually varved sediments were discovered in a large number of non-glacial lakebeds from other parts of the world. This opened for local absolute dating of lake deposits. Many excellent papers were published (e.g. Anthony, 1977; Kelts \& Hsü, 1978; Sturm, 1979; O'Sullivan, 1983; Anderson et al., 1985; Saarnisto, 1985).

\section{A period of revision and extension}

In Sweden and Finland, we entered into a period of revision. The postglacial varves along River Ånermanälven and the connection to the present were revised by Cato (1987), and an error of +350 varves was established. The varves from Central Sweden to Stockholm were revised by Järnefors (1963) and later Strömberg (1989), who found a minor error of +19 varves. The number of varves between the "drainage of the Baltic Ice Lake" and the immigration of saltwater at Stockholm at De Geer's varve -1073 (De Geer, 1940; Mörner, 1995; Johnson et al., 2013) was set at 292 varves by Sauramo (1923), later at 299 varves by Mörner (1977) and finally 310 varves by Brunnberg (1995), who dated the two events at, respectively, 10,740 and 10,430 BP. Kristiansson (1986) extended the chronology through the Younger Dryas and Alleröd periods, with an additional sequence by Ringberg (1991). So, today, the Swedish Varve Chronology spans some 14,000 varves (Fig. 5) with, as it seems, quite a small margin of error in the varve dating. It must be noted, however, that there still remains a significant discrepancy with respect to calibrated C14-ages, which seems to be as much as in the order of 700 years (Mörner, 2003, p. 179) to 800 years (Wohlfart \& Possnert, 2000); the varve ages being too young. The missing varves must be searched for at a time younger than 9663 varves BP, and maybe between 5000 and 2000 BP (Wohlfarth et al., 1997). 
Niemelä (1971) revised the Finnish varve chronology. In Estonia and the St. Petersburg area, there are local varve sequences ("floating" varve chronologies) not yet connected to the Finnish and Swedish time scales (Hang \& Kohv, 2013).

\section{The application of events, spatial distribution and rates}

Varve dating is very useful when it concerns the dating of the duration of a geological event. De Geer (1940) was able to show the mode of ice recession and date esker centra and moraine ridges as to single years (Fig. 2). Varve chronology also gives the background for rate calculations. The classical example is the rate of ice retreat and its changes over time (De Geer, 1940). The rate of ice marginal recession over the Stockholm area was in the order of $300 \mathrm{~m}$ per year, despite the fact that the ice flow to the front was in the order of $500 \mathrm{~m}$ per year, implying a total annual melting of about $700-800 \mathrm{~m}$. This is an enormous rate of ice melting (Fig. 3a). Still, the rate of global sea level rise was in the order of $10 \mathrm{~mm} /$ year. This value is of great significance, because all present-day sea level changes must be well below this value (Mörner, 2011).

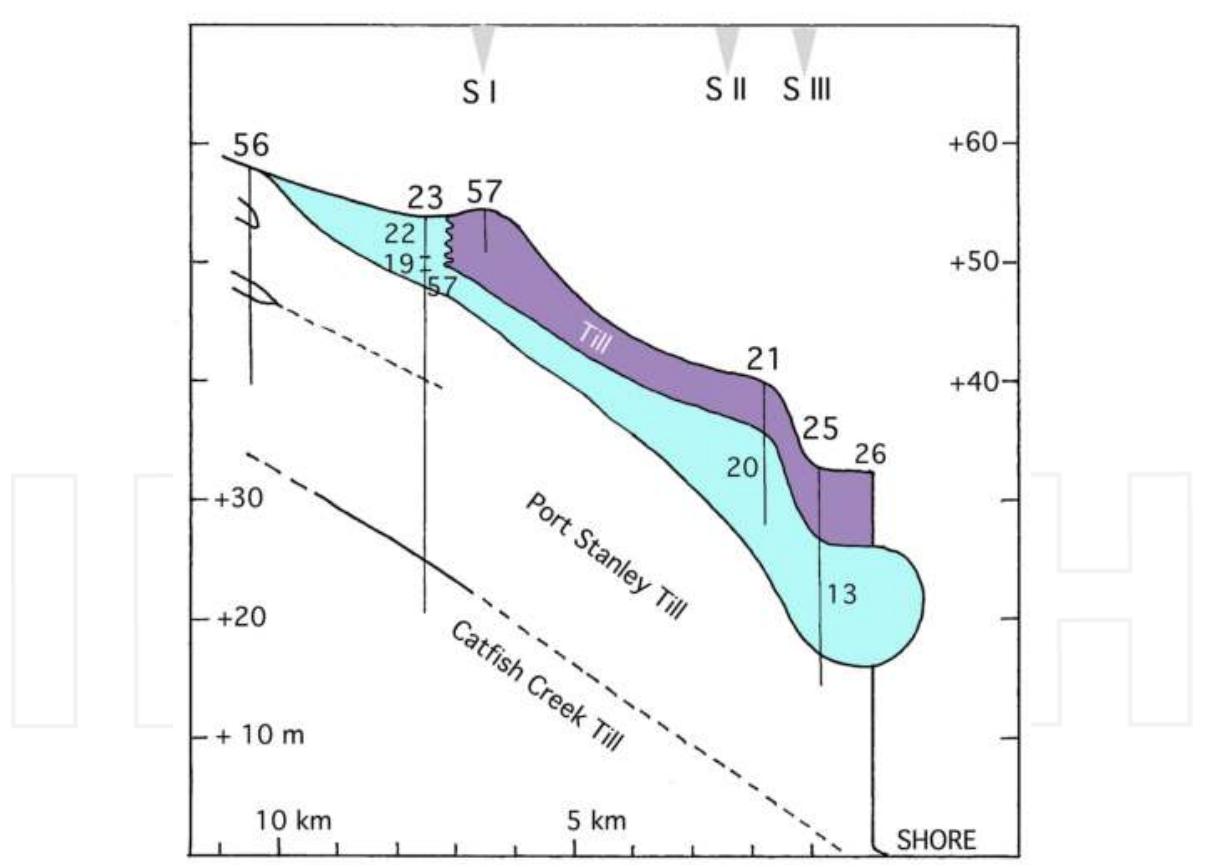

Figure 6. Stratigraphy of the Kettle Creek at the northern shore of Lake Erie including three separate till beds, the last one of which is underlain by varves indicating a readvance of about $8 \mathrm{~km}$ and halt of 22 years for the building up the Tillsonburg-Sparta I (SI) Moraine (Mörner, Ice recession and varve chronology in southern Ontario, unpublished). 
The deglaciation of the Ontario region in Canada is characterized by a number of end moraines representing halts or minor re-advances. By applying a relative varve chronology, it was possible to date the duration of the building up of the Tillsonburg Moraine at 22 years (Fig. 6). A varve sequence right in front of the Tillsonburg-Sparta I end-moraine includes 98 varves; 57 recessional varves, 19 readvance varves and 22 ice-marginal varves. This indicates a readvance in the order of $8 \mathrm{~km}$ and time of ice-marginal halt of only 22 years.

In a few cases it has been possible also to pinpoint the season of an event. This is the case for a major earthquake in Sweden, which was shown to have occurred in the autumn of varve 10,430 BP (Mörner, 2003, 2011, 2013a).

Because different events in Sweden could be tied to one and the same varve, it was possible to document the spatial distribution of those events. This has been especially useful in paleoseismology (Mörner, 2003, 2011, 2013a; Mörner \& Sun, 2008). Turbidites were recorded at single varves and their spatial distribution recorded. There is a relation between seismic magnitude and the spatial distribution of liquefaction. Thanks to the varve chronology in Sweden, a paleoseismic event occurring in varve 10,430 BP was shown to have generated liquefaction over an area of 320x100 km, indicating that this event must have had a magnitude of $>8$ on the Richter scale.

In the glacial varves in the Stockholm region, it was possible to document and date seven separate paleoseismic events within the period 10,490 to 10,388 varves BP (Mörner, 2011); i.e. 7 events in 102 years. This is a very high seismic frequency (or recurrence time). This record could only have been achieved thanks to the firm varve dating.

At Hudiksvall at the coast of central Sweden, the difference in elevation $(7,8 \mathrm{~m})$ and difference in time ( 25 varves) between the Baltic level at the deglaciation and at a tsunami event in varve 9663 BP was known (Mörner, 2003, p. 183). Consequently the relative land uplift must have been in the order of $31 \mathrm{~cm}$ per year (with eustatic calibration corresponding to a rate of absolute uplift in the order of $40 \mathrm{~cm} / \mathrm{yr}$ ). This is a unique value, which provides a very accurate measurement of the rate of uplift right after the free-melting.

Micro-varved postglacial lake sequences occur both in Sweden and Finland. They provide excellent chronological tools for the recording and dating of environmental changes (e.g. Renberg, 1983; Renberg et al., 1984; Ojala \& Tiljander, 2003; Ojala et al., 2008; Ojala et al., 2013). This also includes the recording and dating of secular paleomagnetic changes in the Holocene (Ojala \& Tiljander, 2003). Maier et al. (2013) were able to assess the rate of sediment compaction; after 3-5 years the varve thickness had decreased by $60 \%$.

Lake Kassjön at Umeå in northern Sweden has lake sediments that are annually varved for the last 6300 years. We applied paleomagnetic studies of these deposits (Mörner and Sylwan, 1989). A major swing in declination was recorded at around 2600 varves BP, which is about where the production of ${ }^{14} \mathrm{C}$ records a major spike. Ten samples were $\mathrm{C} 14$-dated over the swing in declination (the same sample as paleomagnetically analysed). Declination swings to the west by $117^{\circ}$ in 348 years, which implies implies a rate of $0.36^{\circ}$ per year. This change constitutes a "trans-polar VGP shift" (Mörner, 1991). It coincides with the main spike in ${ }^{14} \mathrm{C}$-production. Therefore, this event is likely to represent an internal perturbation of the Earth's own geo- 
magnetic field and not Solar Wind driven change of the geomagnetic shielding and ${ }^{14} \mathrm{C}$ production (Mörner, 2013b).

The very long varved core sequence from Lake Suigetsu in Japan extends the C14-calibation back to 45,000 BP (Kitagawa \& van der Plicht, 2000).

Finally, it may be of historical interest to note that Wilson already in 1943 reported on a varvesequence of 12,223 varves from the southwestern part of Lake Erie (Wilson, 1943).

\section{Pre-Quaternary varves}

Varved sediments are, of course, not restricted to the Quaternary period. Glacial varves are recorded for all previous glaciations, too. The Permian varves in Brazil provide fine examples of glacial varves, and have led to the establishment of a special exhibition park known as Parque do Varvito where the varves are excellently preserved (Fig. 7). The Late Precambrian ( 650 Ma) varves of the Elatina Formation in Australia (Williams, 1985) are important because they provide records of a " 12-laminae cycle" interpreted as the 11-yr solar cycle.
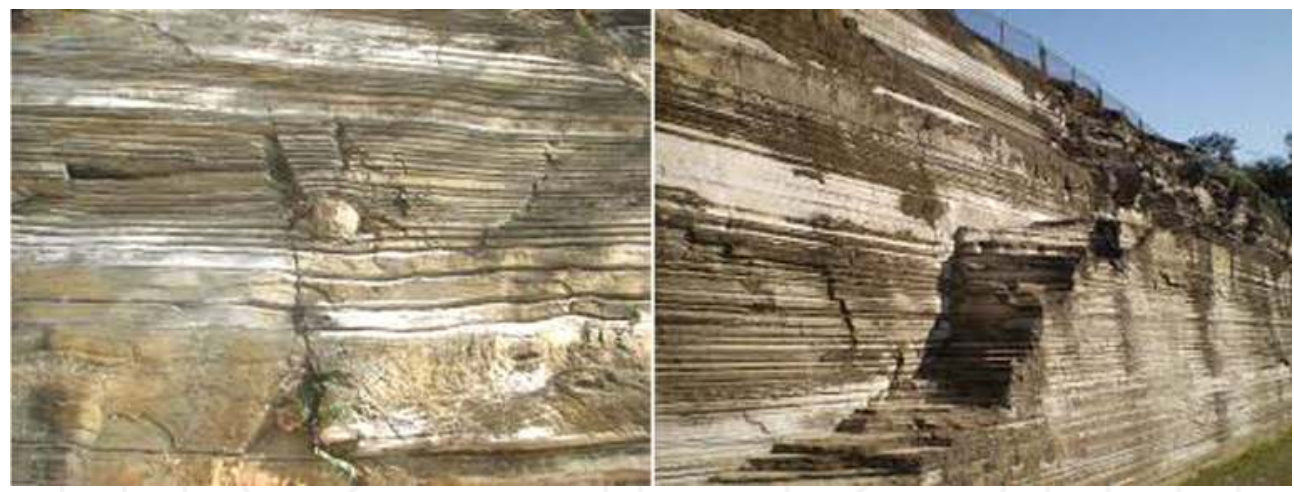

Figure 7. Parque do Varvito exhibiting Permian varves. Right: view of the main sequence. Left: close-up of proximal varves in the centre including an ice-rafted block.

\section{Conclusions}

“The Swedish Varve Chronology" was invented and built up by De Geer (1940). With much revision and addition, the chronology now covers a period of about 14,000 years. It is based on the successive correlation of varve segments representing the deposition of varved clay in front of the receding ice margin (on-lapping varves) plus the postglacial varves of deltaic river varves down the River Ångermanälven (off-lapping varves). The sequence older than about 
9500 varved BP has an error of about 700 missing varves with respect to the radiocarbon calibrated chronology.

Varve records have a great potential when it comes the determinations of durations and rates of a large variety of events recorded by the varves. In this case, the chronology needs not to be fixed to the present, but may also be a "floating chronology" just providing a short sequence of precise annual determination. This applies for all varve records from deposits older than the Last Ice Age (e.g. Williams, 1985).

Continual varve sequences from lakes basins offer local chronologies of very high precision (e.g. Kitagawa \& van der Plicht, 2000; Ojala \& Alenius, 2005), and can be used to date a large number of local environmental changes. Today, this application of varve records seems to be more important (VWG, 2014) than the building up of local chronologies like the famous "Swedish Time Scale" or "Swedish Varve Chronology".

\section{Author details}

Nils-Axel Mörner

Paleogeophysics \& Geodynamics, Sweden

\section{References}

[1] Anderson, R. Y., Dean, W. E., Bradbury, J. P. \& Love, D. (1985). Meromictic lakes and varved lake sediments in North America, U. S. Geological Survey Bulletin, 1607, 1-19.

[2] Anthony, R. S. (1977). Iron-rich rhythmically laminated sediments in Lake of the Clouds, northeastern Minnesota, Limnology and Oceanography, 22, 45-54.

[3] Arnold, J. R. \& Libby, W. F. (1949). Age determination by radiocarbon content: Check with samples of known age, Science, 110, 678-680.

[4] Antevs, E. (1932). Late-glacial clay chronology of North America, Ann. Rep. Smithsonian Inst., 1931, 313-324.

[5] Bergström, R. (1968). Stratigrafi och isrecesion i södra Västerbotten. Sveriges Geologiska Undersökning, C 634, 1-76.

[6] Brunnberg, L. (1995). Clay-varve chronology and deglaciation during the Younger Dryas and Preboreal in the easternmost part of the Middle Swedish ice marginal zone, Ph.D. thesis, Stockholm University, Quaternaria, Series A 2, 1-94. 
[7] Caldenius, C. C:zon (1932). Las glaciaciones Cuaternarias en la Patagonia y Tierra del Fuego, Geogr. Ann., 1932, 1-164.

[8] Cato, I. (1987). On the definitive connection of the Swedish Time Scale with the present, Sveriges Geol. Undersökning, Ca-68, 1-55.

[9] De Geer, G. (1884). Lecture at the Geological Society in Stockholm on "ishavslerans varvighet", Geol. Fören. Stockholm Förhandl., 7.

[10] De Geer, G. (1912) A geochronology of the last 12,000 years, Compte Rendus XI Session du Congree Geologique International, 241-253, Stockholm.

[11] De Geer, G. (1940) Geochronologia Suecia Principles, Kungliga Svenska Vetenskapsakademiens Handlingar, 3rd 18 (6), 1-360.

[12] Francus, P., Ridge, J.C. \& Johnson, M.D. (2013). The rise of varves, GFF, 135 (3-4), 229-230.

[13] Fromm, E. (1938). Geochronologisch datierte Pollendiagramme und Diatoméenanalysen aus Ångermanland, Geologiska Föreningens $i$ Stockholm Förhandlingar, 60, 365-381.

[14] Gutenberg, B. (1941). Changes in sea level, postglacial uplift, and mobility of the Earth's interior, Bull. Geol. Soc. Amer., 52, 721-772.

[15] Hang, T. \& Kohv, M. (2013). Glacial varves at Pärnu, south-west Estonia: a local varve chronology and proglacial sedimentary environment, GFF, 135 (3-4), 273-281.

[16] Högbom, A.G. (1889). Om relationen mellan kalcium-och magnesiumkarbonat i qvartära aflagringar. Geologiska Föreningens i Stockholm Förhandlingar, 11, 263-273.

[17] Johnson, M.D., Kylander,M.E., Casserstedt, L. Wiborgh, H. \& Björck, S. (2013). Varved glaciomarine clay in central Sweden before and after the Baltic Ice Lake drainage: a further clue to the drainage events at Mt. Billingen, GFF, 135 (3-4), 293-307.

[18] Johnston, W.A. (1922). Sedimentation in Lake Louise, Alberta, Canada, American J. Sci., 5 (4), 376-386.

[19] Järnefors, B. (1963). Lervarvskronologien och isrecessionen I östra Mellansverige, Sveriges Geol. Undersök., C-594, 1-67.

[20] Kelts, K. \& Hs ๑, K. J. (1978). Freshwater carbonate sedimentation. In: Lakes: Geology, Chemistry, Physics (A. Lerman, Ed.), 295-325, Springer-Verlag.

[21] Kitagawa, H. \& van der Plicht, J. (2000). Atmospheric radiocarbon calibration beyond 11,900 CAL BP from Lake Suigetsu laminated sediments, Radiocarbon, 42, 370-381.

[22] Kristiansson, J. (1986). The ice recession in the southeastern part of Sweden. A varvechronological time scale for the latest part of the Late Weichselian, Ph.D. thesis, Stockholm University, Kvartärgeol. Inst., Rap. 7, 1-132. 
[23] Kuenen, P.H. (1951). Mechanisms of varve formation and the action of turbidity currents. Geol. Fören. Stockh. Förhandl., 73, 69-83.

[24] Lidén, R. (1913). Geokronologiska studier öfver det finiglaciala skedet I Ångermanland, Sveriges Geol. Undersökn., Ca-9, 1-39.

[25] Lidén, R. (1938). Den senkvartära strandförskjutningens förlopp och kronologi i Ångermanland, Geologiska Föreningens i Stockholm Förhandlingar, 60, 397-404.

[26] Maier, B., Rydberg, J., Bigler, C. \& Renberg, I. (2013). Compaction of recent varved sediments, GFF, 135 (3-4), 231-236.

[27] Mörner, N.-A. (1977). Varve Chronology on Södertörn: recording and dating of the "drainage" of the Baltic Ice Lake and correlation with the Finnish varve chronology, Geologiska Föreningens i Stockholm Förhandlingar, 99 (4), 423-425.

[28] Mörner, N.-A. (1978). Varves and varved clays, Encyclopedia of the Earth Sciences, VIA Sedimentology (R.W. Fairbridge, ed.), 841-843.

[29] Mörner, N.-A. (1979). The Fennoscandian uplift and Late Cenozoic geodynamics: geological evidence, GeoJournal, 3 (3), 287-318.

[30] Mörner, N.-A. (1991). Trans-polar VGP shifts and Earth's rotation, Geophys. Astrophys. Fluid Dynamics, 60, 149-155.

[31] Mörner, N.-A. (1995). The Baltic ice lake e Yoldia Sea transition, Quaternary International, 27, 95-98.

[32] Mörner, N.-A. (2003). Paleoseismicity of Sweden - a novel paradigm. A contribution to INQUA from its Sub-commission on Paleoseismology, Reno 2003, 320 pp., P\&Gprint, Stockholm. ISBN-91-631-4072-1.

[33] Mörner, N.-A. (2011). Setting the frames of expected future sea level changes, In: Evidence-based Climate Science (D.J. Easterbrook, ed.), Chapter 6, 197-209, Elsevier.

[34] Mörner, N.-A. (2013a). Drainage varves, seismites and tsunamites in the Swedish varve chronology, GFF, 135 (3-4), 308-315.

[35] Mörner, N.-A. (2013b). Planetary beat and solar-terrestrial responses, Pattern Recogn. Physics, 1, 107-116.

[36] Mörner, N.-A. \& Sun., G. (2008). Paleoearthquake deformations recorded by magnetic variables, Earth and Planetary Science Letters, 267, 495-502.

[37] Mörner, N.-A. \& Sylwan, C. (1989). Detailed paleomagnetic record for the last 6300 years from varved lake deposits in northern Sweden. In: Geomagnetism and Paleomagnetism (F. Lowes et al., Eds.), p. 63-70. Kluwer, Dordrecht. 
[38] Niemelä, J. (1971). Die quartäre Sratigraphie von Tonablagerungen und der Rückzug des Inlandseises zwischen Helsinki und Hämeenlinna in Füdfinnland, Geol. Surv. Finland, Bull. 253, 1-79.

[39] Ojala, A.E.K. \& Tiljander, M. (2003). Testing the fidelity of sediment chronology: comparison of varve and paleomagnetic results from Holocene lake sediments from central Finland, Quaternary Sci. Reviews, 22, 1787-1903.

[40] Ojala, A.E.K. \& Alenius, T. (2005). 10000 years of interannual sedimentation recorded in the Lake Nautajärvi (Finland) clastic-organic varves, Palaeogeogr. Palaeoclim. Palaeoecol., 219, 285-302.

[41] Ojala, A.E.K., Heinsalu, A., Kauppila, T., Alenius, T. \& Saarnisto, M. (2008). Charactaerizing changes in the sedimentary environment of a varved lake sediment record in southern central Finland around 8000 cal. yr BP, J. Quaternary Sci., 23 (8), 765-775.

[42] Ojala, A.E.K., Kosonen, E., Weckström, J., Korkonen, S. \& Korhola, A. (2013). Seasonal formation of clastic-biogenic varves: the potential for palaeoenvironmental interpretations, GFF, 135 (3-4), 237-247.

[43] O'Sullivan, G. (1983). Annually laminated lake sediments and the study of Quaternary environmental changes, Quaternary Sci. Rev., 1, 245-313.

[44] Renberg, I. (1983). Varved lake sediments - geochronological records of the Holocene, Geologiska Föreningens i Stockholm Förhandlingar, 104, 275-279.

[45] Renberg, I., Segerström, U. \& Wallin, J.-E. (1984). Climatic reflection in varved slake sediments, In: Climate Changes on a Yearly to Millennial Basis (N.-A. Mörner \& W. Karlén, Eds.), 249-256, Reidel Publ. Co.

[46] Ridge, J.C. and Larsen, F.D. (1990). Re-evaluation of Antevs' New England varve chronology and new radiocarbon dates of sediments GlacialLake Hitchcock, Geol. Soc. Am. Bull., 102, 889/899.

[47] Ringberg, B. (1984). Cyclic lamination in proximal varves reflecting the length of summers during the Late Weichsel in southernmost Sweden. In: Climate Changes on a Yearly to Millennial Basis (N.-A. Mörner \& W. Karlén, Eds.), 57-62, Reidel Publ. Co.

[48] Ringberg, B. (1991). Late Weichselian clay varve chronology and glaciolacustrine environment during deglaciation in southeastern Sweden, Sveriges Geol. Undersökning, Ca-79, 1-42.

[49] Saarnisto, M. (1985). Long varve series in Finland, Boreas, 14, 133-137.

[50] Sauramo, M. (1923). Studies on the Quaternary varve sediments in southern Finland, Bull. Comm. Géol. Finlande, 60, 00-00.

[51] Strömberg, B. (1989). Late Weichselian deglaciation and clay-varve chronology in east-central Sweden, Sveriges Geologiska Undersökning, Ca 73, 1-70. 
[52] Sturm, M. (1979). Origin and composition of clastic varves. In: Moraines and varves (C, Schlüchter, Ed.), 281-285, Balkema.

[53] Tröften, P.E. \& Mörner, N.-A. (1997). Varved clay chronology as a means of recording paleoseismic events in southern Sweden, Journal of Geodynamics, 24, 249-258.

[54] VWG (Varves Working Group) (2014).

[55] Williams, G.E. (1985). Solar affinity of sedimentary cycles in the Late Precambrian Elatina Formation, Austr. J. Physics, 38, 1027-1043.

[56] Wilson, I.T. (1943). Varves in Sandusky Bay's sediments, Ohio J. Sci., 43,, 195-197.

[57] Wohlfart, B., Björck, S., Cato, I. \& Possnert, G. (1997). A new middle Holocene varve diagram from the river Ånegermanlandälven, northern Sweden: indications of a possible error in the Holocene varve chronology, Boreas, 26, 347-353.

[58] Wohlfart, B. \& Possnert, G. (2000). AMS radiocarbon measurements from the Swedish varved clays, Radiocarbon, 42, 323-333.
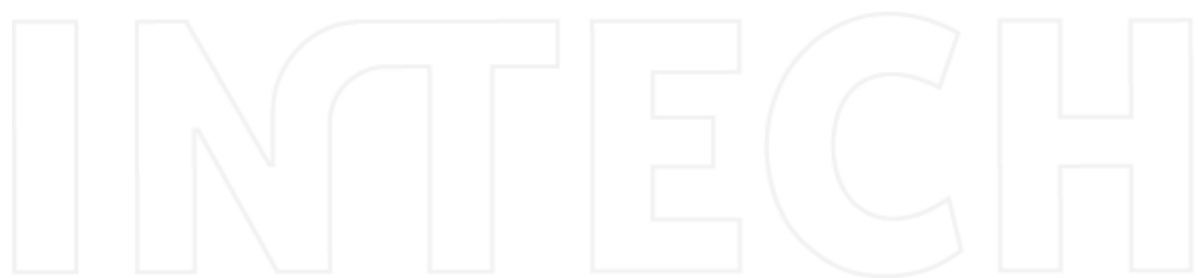

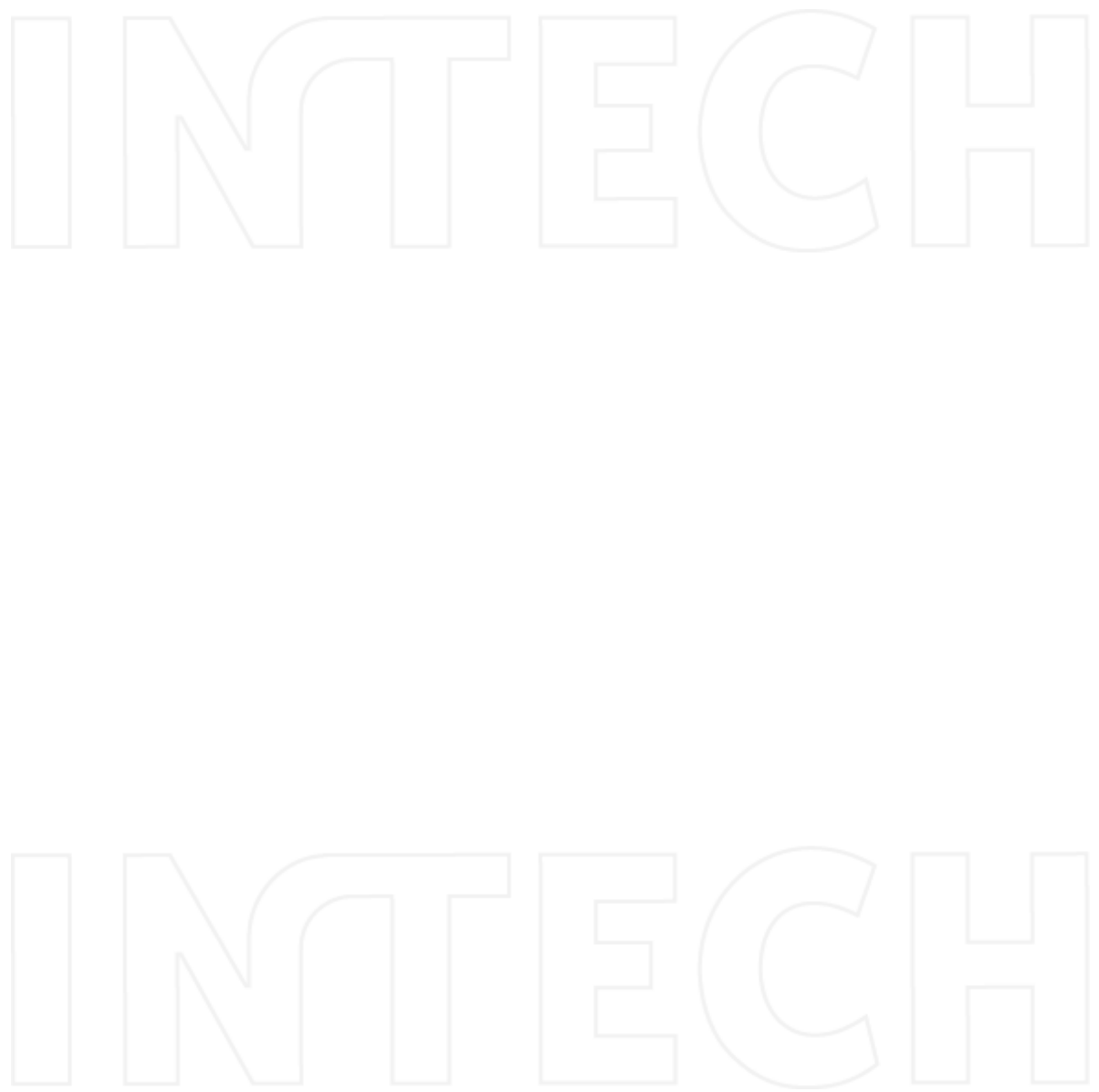


\section{Chapter 4}

\section{Geochronology From The Castelo Branco Pluton (Portugal) - Isotopic Methodologies}

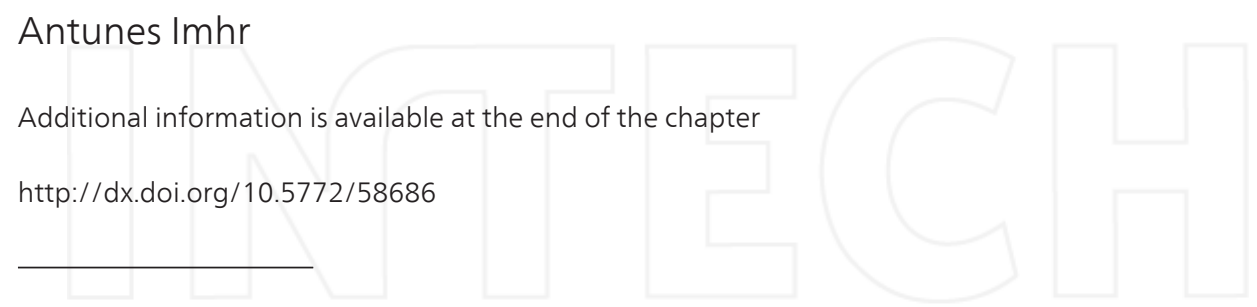

\section{Introduction}

The geochronology of granitic rocks is a key-issue in the crustal evolution and orogenic processes. Modern high precision techniques have been used to identify relevant geological episodes.

$\mathrm{U}-\mathrm{Th}-\mathrm{Pb}$ chemical dating by electron-microprobe (EPMA) is a potentially valuable method in monazite-bearing rocks. Monazite presents the fundamental conditions required to apply this procedures: 1) monazite is a U-Th enriched phase, 2) all $\mathrm{Pb}$ monazite is radiogenic; 3 ) its closure temperature has proved to be fairly high, up to $900^{\circ} \mathrm{C}$ [1] and 4) the system remains close [2]. Monazite presents a higher resistance than zircon to radiation damage effects [3] and low diffusion rates [4].

Different studies have demonstrated that U-Th-Pb dating by EPMA is an accurate method of geochronology (e.g., [5-8]). U-Th-Pb monazite age determination can be obtained in small crystals $(5 \mu \mathrm{m})$, allowing the study of mineral heterogeneities, without destruction and preserving textural relationships. Microanalytical techniques are an adequate way to study magmatic and polymetamorphic events registered in monazites with zoning textures (e.g., [2, 9-11]). The advantages of this technique are the high spatial resolution and the possibility to obtain rapidly a large number of ages. The main disadvantage is the low accuracy, conditioned by $\mathrm{Pb}$ content and statistical treatment of data. The analytical error frequently ranges from \pm 40 to $\pm 120 \mathrm{Ma}(2 \sigma)$ for ages of 300 to $3000 \mathrm{Ma}$, respectively [8]. However, a statistical treatment of homogeneous ages promotes a decrease of uncertainty to $\pm 20-30 \mathrm{Ma}$ [2].

Attemps to constraint the timing of high-temperature orogenic processes including crustal melting, metamorphism and deformation are typically based upon U-Pb age analysis of accessory minerals such as zircon and monazite. Although growth and recrystallization of 
accessory minerals is being increasingly better understood in the context of the host rock petrogenesis [12-13], it remains a difficult task to related ages measured from zircon and monazite to specific orogenic events. Zircon is a very robust mineral during magmatic and metamporphic events. Very slow rates for $\mathrm{U}$, Th and $\mathrm{Pb}$ help ensure that $\mathrm{U}-\mathrm{Pb}$ age and stable isotopic and trace element compositions are preserved during subsequent deep crustal evolution. Conversely, new zircon growth associated with tectonic events post-dating the initial zircon growth typically occurs at very fine spatial scales [14]. Zircon is capable of preserving a long history of growth and modification.

Monazite also contains valuable chemical and textural information, but tends to recrystallize more readly than zircon [15-16] and thus tend to record age and stable isotopic data that are substantially different from that yielded by coexisting zircon.

ID-TIMS U-Pb age for zircon and monazite is a more accurate and precise methodology and has widely been applied. Uranium decay produces radiogenic $\mathrm{Pb}\left({ }^{207} \mathrm{~Pb}\right.$ and $\left.{ }^{206} \mathrm{~Pb}\right)$, allowing to two independent age results- ${ }^{207} \mathrm{~Pb} /{ }^{235} \mathrm{U}$ and ${ }^{206} \mathrm{~Pb} /{ }^{238} \mathrm{U}$-and a dependent one- ${ }^{207} \mathrm{~Pb} /{ }^{206} \mathrm{~Pb}$ [17]. Data ages were plotted on the conventional U-Pb concordia diagrams and the three obtained ages from the same mineral allows a high precision to the U-Pb system.

Radiogenic isotope ratios are commonly used as petrogenetic tracers, yielding information on time-integrated element fractionation through processes of melting, crystallization, metamorphism and contamination. The Rb-Sr dating method is based on the behavior of two mobile elements and the $\mathrm{Rb}-\mathrm{Sr}$ isotope systematic of igneous rocks can be easily disturbed by fluid infiltration or during a thermal event. Isotopic $\mathrm{Rb} / \mathrm{Sr}$ and $\mathrm{Sm} / \mathrm{Nd}$ data are petrogenetic indicators. Initial ${ }^{87} \mathrm{Sr} /{ }^{86} \mathrm{Sr}$ isotopic ratio and $\varepsilon \mathrm{Nd}_{\mathrm{T}}$ value of magma are a source signature and remain constant during fractionation processes [17].

Whole rock oxygen isotope $\left(\delta^{18} \mathrm{O}\right)$ of granitic rocks will give informations about magma origin and associated magmatic crystallization and assimilation processes. Generally, magmas with no supracrustal input have an uniform oxygen isotope ratio that is distinct from magmas that assimilated or were generated directly from supracrustal sources. However, $\delta^{18} \mathrm{O}$ can show small variations in the magmatic differentiation processes [18-24].

The Castelo Branco pluton consists of five different peraluminous granitic rocks arranged in a concentrically zoned structure [25]. This work will present the different isotopic data (U-Th$\mathrm{Pb}, \mathrm{U}-\mathrm{Pb},{ }^{87} \mathrm{Sr} /{ }^{86} \mathrm{Sr}_{\mathrm{i}}, \varepsilon \mathrm{Nd}_{\mathrm{T}}$ and $\delta^{18} \mathrm{O}$ ) obtained in these granitic rocks to determine their age and protolith information.

\section{Castelo Branco Pluton}

The Castelo Branco granitic pluton is located within the Central Iberian Zone (CIZ), the innermost zone of the Iberian Variscan Belt. This pluton is exposed over an area of $390 \mathrm{~km}^{2}$, with a mean diameter of $19 \mathrm{~km}$, and consists of five late-tectonic Variscan granitic bodies, intruding the schist-greywacke complex (CXG) (Fig. 1). In the NE border, it contacts with a medium-grained biotite granodiorite of $480 \pm 2 \mathrm{Ma}$ [26]. 
The Castelo Branco pluton generated a contact metamorphic aureole up to $2 \mathrm{~km}$ wide, with metasediments recrystallized as pelitic hornfels in the inner zone, and as micaschists in the outer zone. The schist-greywacke complex and the granitic rocks are cut by aplite-pegmatite dikes and quartz veins.

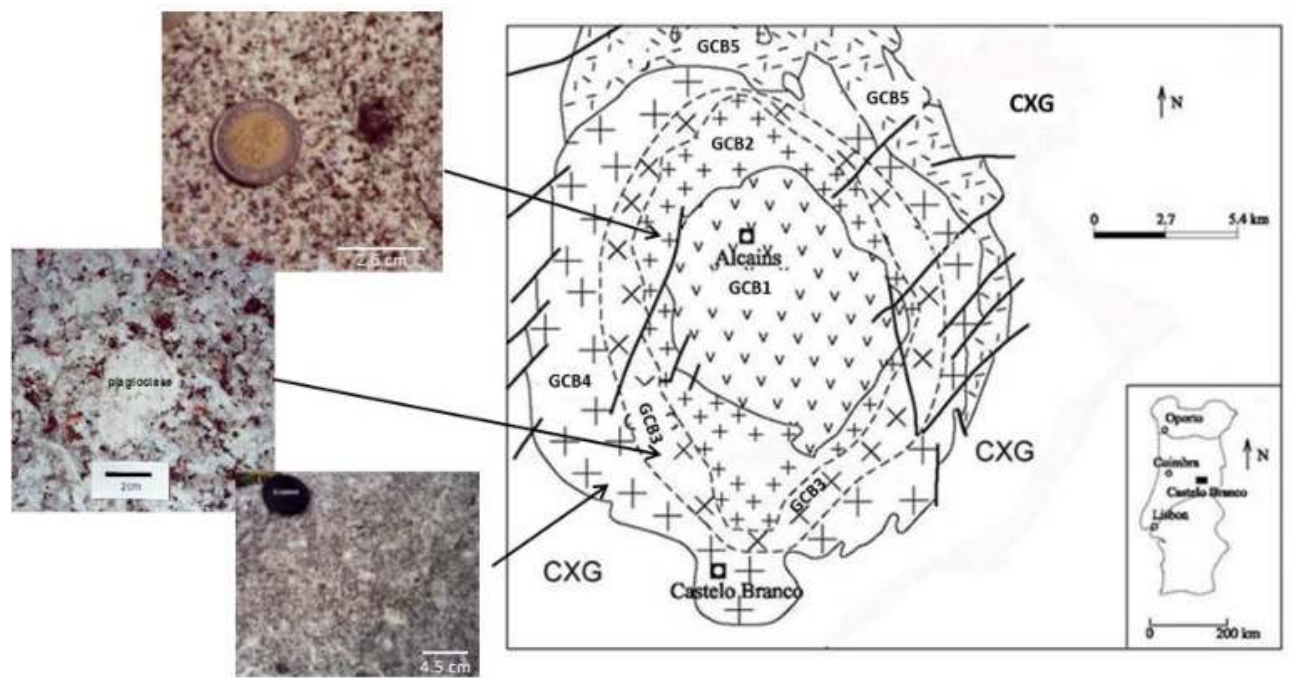

Figure 1. Geographycal setting and geological map of the Castelo Branco pluton. CXG schist-greywacke complex; GCB1 muscovite > biotite granite; GCB2 biotite > muscovite granodiorite; GCB3 biotite > muscovite granodiorite; GCB4 biotite=muscovite granite; GCB5 muscovite > biotite granite.

The medium-to fine-grained muscovite > biotite granite (GCB1) occurs at the pluton's core and is encircled by a medium-to fine-grained, slightly porphyritic, biotite $>$ muscovite granodiorite (GCB2), surrounded by a medium-to coarse-grained porphyritic biotite $>$ muscovite granodiorite (GCB3), which passes gradually to the medium-to coarse-grained, porphyritic biotite=muscovite granite (GCB4). Rounded enclaves of granodiorite GCB2 can be founded in the granodiorite GCB3 and granite GCB4. The boundary of the pluton is limited to N and NE by a coarse-grained muscovite > biotite granite (GCB5) (Fig. 1). The contact between granite GCB1 and granodiorite GCB2 is sharp, as is the contact between granites GCB4 and GCB5. The pluton consists of five granites concentrically distributed of $310 \mathrm{Ma} \pm 1$ [25].

All the granitic rocks from the Castelo Branco pluton contain quartz, microcline, plagioclase, biotite, some chlorite, muscovite, tourmaline, monazite, apatite, zircon, ilmenite and rutile. Zircon and monazite are accessory minerals mainly included in apatite, biotite and plagioclase. Zircon occurs as euhedral crystals, whereas monazites are rounded and easily identified by their pleochroic halos.

The granitic rocks GCB1, GCB2 and GCB5 correspond to three distinct magmatic pulses derived by partial melting of heterogeneous metasedimentary materials. Granodiorite GCB3 
and granite GCB4 result by fractional crystallization of plagioclase, quartz, biotite and ilmenite from the granodiorite GCB2 magma [25].

\section{Analytical methodologies}

\subsection{U-Th-Pb EPMA}

The U-Th- $\mathrm{Pb}$ monazite ages were calculated using $\mathrm{U}, \mathrm{Pb}$ and $\mathrm{Th}$ monazite contents determined by electron-microprobe (EPMA). In general, monazite incorporates large amounts of Th and $\mathrm{U}$ during the rock formation and retains $\mathrm{Pb}$ of the radioactive decay processes. The obtained age will be valid if at the time of formation of the mineral, initial $\mathrm{Pb}$ is practically non-existent and there was no loss of $\mathrm{Pb}$, Th and $\mathrm{U}[6]$.

The $\mathrm{U}, \mathrm{Pb}$ and Th monazite contents of representative samples from the Castelo Branco pluton (Fig. 2) were determined by electron-microprobe on polished thin sections, in a Cameca SX100 at the Laboratoire of Magmas and Volcans, University Blaise Pascal (Clermont-Ferrand, France). The analytical conditions included an accelerating voltage of $15 \mathrm{kV}$ and a beam current of $150 \mathrm{nA}$. The standards used were as follows: $\mathrm{UO}_{2}(\mathrm{U} \mathrm{M} \beta), \mathrm{ThO}_{2}(\mathrm{Th} \mathrm{M} \alpha)$, apatite $(\mathrm{Ca} \mathrm{K} \alpha, \mathrm{P}$ $\mathrm{K} \alpha$ ), zircon ( $\mathrm{Si} \mathrm{K} \alpha$ ) and polysintetic phosphates ( $\mathrm{Y} \mathrm{L} \alpha, \mathrm{La} \mathrm{L} \alpha, \mathrm{Ce} \mathrm{L} \alpha, \operatorname{Pr} \mathrm{L} \beta, \mathrm{Nd} \mathrm{L} \beta, \operatorname{Sm~L} \beta, \mathrm{Gd}$ $\mathrm{L} \beta$ ). The monazite age is directly dependent on the concentrations of $\mathrm{U}$, Th and $\mathrm{Pb}$ and its detection limit was calculated [27], with an error of $2 \sigma$ associated with the uncertainty of these elements (for a confidence level of $95 \%$ ) in the equation decay. The treatment of individual analysis, including $\mathrm{Th}, \mathrm{U}$ and $\mathrm{Pb}$ contents of each monazite crystals, have been perfomed using a GwBasic computer program providing an age for each individual analysis. A statistical treatment giving the corresponding age to the studied population and the values of the sum of squared deviations (MSWD) is used for the results validation [6, 28]. If the system remained close since the early stages of crystallization, the obtained MSWD value will be less or equal to 1 , whereas if the system changed flowing through interactions between minerals can promote recrystallization processes, and the MSWD value greatly increases [29].

\section{2. $\mathrm{U}-\mathrm{Pb}$ zircon and monazite ages}

The U-Pb geochronological ages were proceeded with the preparation of zircon and monazite concentrates from representative samples of the Castelo Branco pluton. Zircon and monazite separation was carried out by a combination of magnetic separation and heavy liquids.

The preparation of the selected samples (20 to $25 \mathrm{~kg}$ per sample), included grinding, sieving and separation of the different granulometric fractions. After this, a subsample corresponding to the fraction below 180 mesh was selected and contains the majority of zircon and other accessory minerals. Subsequently, this subsample passed through a magnetic separator in a vertical position with a maximum speed to separate the more magnetic minerals (e.g. biotite) from the remaining fraction.

Otherwise, the less magnetic fraction is placed in a glass ampoule containing bromoform $(\mathrm{d}=2.81)$ to recover the heavy concentrated sample which was washed with purified water and 

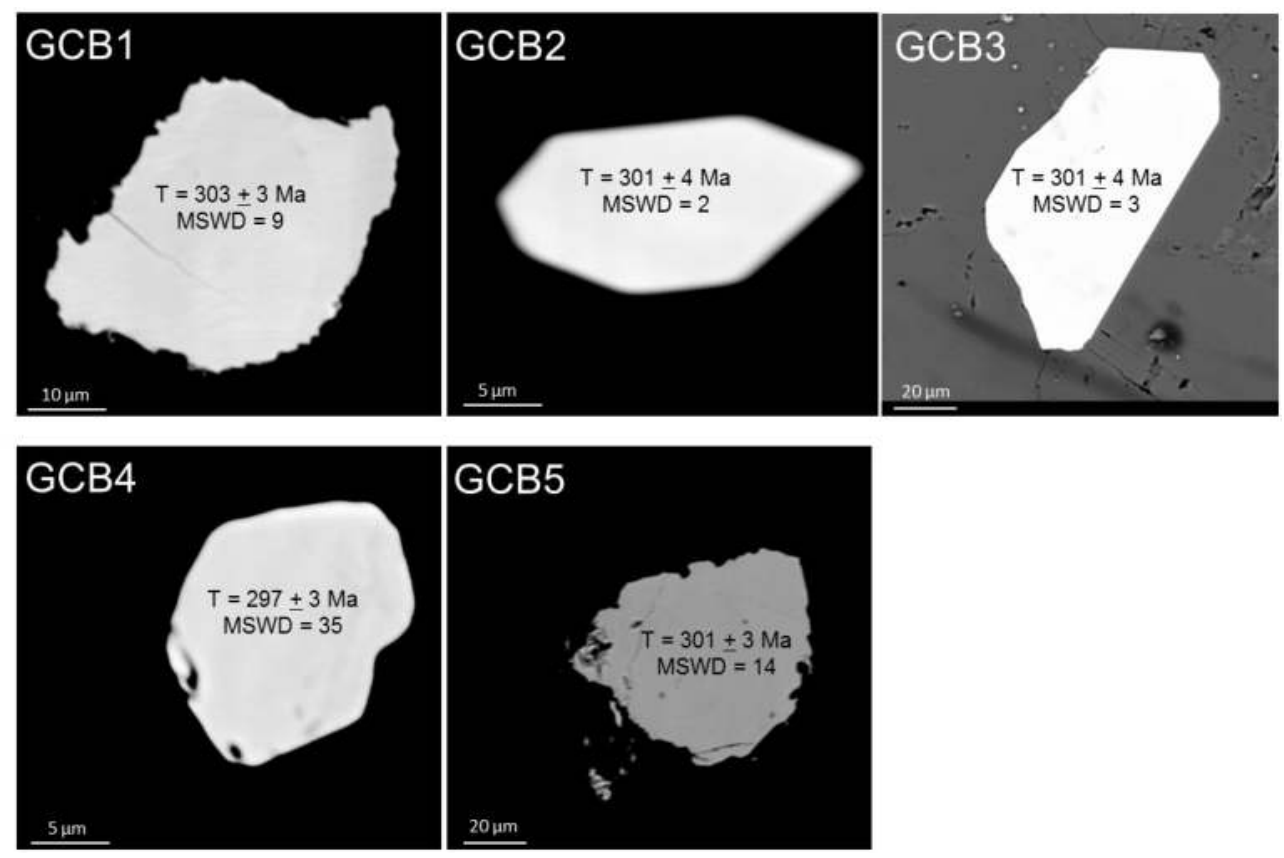

$\overline{\mathrm{GCB} 1, \mathrm{GCB} 2, \mathrm{GCB} 3}, \mathrm{GCB} 4$ and GCB5 are those in Figure 1.

Figure 2. U-Th-Pb monazite ages from granitic rocks of Castelo Branco pluton.

acetone, and dried in an oven. After this, the concentrated sample was separated with methylene iodide $(\mathrm{d}=3.3)$ and the heavier fraction, which contains zircon and monazite, was washed with acetone and distilled water to eliminate methylene iodide wastes. At the end, the heavy concentrate sample was passed through a magnetic separator and different magnetic fractions containing monazite $(0.8$ to $1.0 \mathrm{~A})$ and zircon $(\geq 1.7 \mathrm{~A})$ were obtained. All the methodology must be carefully followed and will be fundamental for the quality of zircon concordia diagrams [30-32]. A consistent zircon concordia diagram requires non-magnetic zircon concentrates because magnetic ones are also rich in uranium, and therefore become the most likely to lost radiogenic lead and, consequently are more discordant [30].

Monazite grains for $\mathrm{U}-\mathrm{Pb}$ analysis are selected from the concentrated magnetic fraction and grains free of cracks and inclusions should be used, like as to selected zircon grains. Representative crystals of zircon and monazite populations are selected by hand-picking, avoiding the fractured ones or with inclusions and, if possible, of inherited cores. However, these inherited cores are not always detectable by binocular or even optical microscope.

Selected frations of zircon and monazite were submitted to air-abrasion to prevent fracturation of the minerals, remove external portions and possible disturbances [30, 33].

The abraded crystals are washed with $\mathrm{HNO}_{3}(4 \mathrm{~N}), \mathrm{H}_{2} \mathrm{O}$ and acetone, weighted and added "spike" ${ }^{205} \mathrm{~Pb} /{ }^{235} \mathrm{U}$ to dissolution processes. Zircon is dissolved with $\mathrm{HF}\left(+\mathrm{HNO}_{3}\right)$ using teflon 
microcapsules and heated to $185^{\circ} \mathrm{C}$ [34], whereas monazite is dissolved with $\mathrm{HCl}(6 \mathrm{~N})$ in savillex containers on a hotplate. After evaporation, $\mathrm{HCl}(3.1 \mathrm{~N})$ is added to each microcapsule and savillex containers, and the solution is passed through a $\mathrm{HCl}$ ion exchange column resin to purify $\mathrm{U}$ and $\mathrm{Pb}$. Finally, the crystals and blank samples are placed on the filament by adding 2 drops of $\mathrm{H}_{3} \mathrm{PO}_{4}$ and silica gel.

The $\mathrm{U}-\mathrm{Pb}$ isotopic results for zircon and monazite were obtained by isotope dilution thermal ionization mass spectrometry (ID-TIMS) using a Finnigan Mat 262 spectrometer at the Department of Geosciences, University of Oslo, Norway [34-37]. The initial Pb correction was done using model compositions [38] and decay constants [39]. The Isoplot program [40] was used for the plots and regression. All uncertainties relative to the analyses and ages are given at the $2 \sigma$ level.

\section{3. $\mathrm{Rb}-\mathrm{Sr}, \mathrm{Sm}-\mathrm{Nd}$ and $\delta^{18} \mathrm{O}$ whole rock}

The Sr and Nd isotope analyses were obtained at the Centro de Instrumentación Científica of the University of Granada, Spain. Samples were digested using ultraclean reagents and analyzed by thermal ionization mass spectrometry (TIMS), using a Finnigan Mat 262 spectrometer, after chromatographic separation with ion-exchange resins [41].

The standardized ratios ${ }^{86} \mathrm{Sr} /{ }^{88} \mathrm{Sr}=0.1194$ and ${ }^{146} \mathrm{Nd} /{ }^{144} \mathrm{Nd}=0.7219$ and blank values for $\mathrm{Sr}$ and $\mathrm{Nd}$ were of 0.6 and $0.09 \mathrm{ng}$, respectively. The external precision of the method $(2 \sigma)$ was estimated by ten successive samples corresponding to different attacks in the same standard sample WSE [42]. This value can be considered as a laboratory error, since it includes both the different attacks and chemical separation of the various samples, as well as the instrumental error of the determinations. The obtained errors were ${ }^{87} \mathrm{Sr} /{ }^{86} \mathrm{Sr}=0.706596 \pm 0.000018(2 \sigma=0.0026$ $\%)$ and ${ }^{143} \mathrm{Nd} /{ }^{144} \mathrm{Nd}=0.512467 \pm 0.0000084(2 \sigma=0.0016 \%)$. On the analysis, the NBS 987 was used as a standard solution, with a reproducibility for repeated measurements of ${ }^{87} \mathrm{Sr} /{ }^{86} \mathrm{Sr}=0.71025$ $\pm 0.0000046(2 \sigma=0.0065 \%)$. The reproducibility obtained for the La Jolla standard was ${ }^{143} \mathrm{Nd} /$ ${ }^{144} \mathrm{Nd}=0.511845 \pm 0.0000072(2 \sigma=0.0014 \%)$. The isotopic ratios ${ }^{87} \mathrm{Rb} /{ }^{86} \mathrm{Sr}$ and ${ }^{147} \mathrm{Sm} /{ }^{144} \mathrm{Nd}$ were determined with an accuracy better than $\pm 1.2 \%$ and $\pm 0.9 \%(2 \sigma)$, respectively. Regression lines of ${ }^{87} \mathrm{Rb} /{ }^{86} \mathrm{Sr}$ versus ${ }^{87} \mathrm{Sr} /{ }^{86} \mathrm{Sr}$ were calculated using the least-squares method [43], implemented in the Isoplot program [40]. Errors are quoted at the 95\% confidence level and are $2 \sigma$.

The isotopic results of $\delta^{18} \mathrm{O}$ whole rock of the five granitic rocks were obtained in the Department of Earth Sciences, University of Western Ontario (Canada), using conventional extraction line and trifluoride chlorine as a reactant. This method has an accuracy of $\pm 0.2 \%$ and patterns such as quartz and $\mathrm{CO}_{2}$ laboratory were used.

\section{Isotopic geochronology}

\subsection{U-Th-Pb (EPMA) monazite ages}

$\mathrm{U}-\mathrm{Th}-\mathrm{Pb}$ contents of selected monazite grains from the five granitic rocks of the Castelo Branco pluton were determined by EPMA and calculated monazite ages (Table I). A total of 195 U- 
Th- $\mathrm{Pb}$ analyses (EPMA) of monazite were obtained from the granitic rocks of the Castelo Branco pluton. Monazite crystals are homogeneous and unzoned (Fig. 2) and a distinct age between core and rim was not found. Therefore, an individual age were considered to the analysed grains.

Monazites from GCB1 present the highest $\mathrm{Pb}$ and $\mathrm{U}$ contents of granitic rocks from the Castelo Branco pluton (Table I). Monazite age data obtained from two samples of GCB1 granite apparently show a great dispersion, but if the errors obtained are taken into account the ages are similar. Lead, $\mathrm{U}$ and Th average contents from monazite crystals of granodiorites GCB2 and GCB3 and granite GCB4 show variation but within a similar range (Table I). Otherwise, monazite from granite $\mathrm{GCB} 5$ contains higher $\mathrm{Pb}$ and $\mathrm{U}$ contents than monazites from granodiorites GCB2 and GCB3 and granite GCB4 (Table 1).

\begin{tabular}{|c|c|c|c|c|c|c|c|c|}
\hline \multirow[t]{2}{*}{ Sample } & \multirow{2}{*}{$\begin{array}{c}\mathrm{Pb}(\mathrm{ppm}) \\
\pm \sigma\end{array}$} & \multirow{2}{*}{$\begin{array}{c}\mathrm{U}(\mathrm{ppm}) \\
\pm \sigma\end{array}$} & \multirow{2}{*}{$\begin{array}{c}\text { Th (ppm) } \\
\pm \sigma\end{array}$} & \multirow{2}{*}{$\begin{array}{l}\mathrm{T} \text { (Ma) } \\
\pm \sigma\end{array}$} & \multirow[t]{2}{*}{ N } & \multicolumn{3}{|c|}{ Average Age } \\
\hline & & & & & & $\mathrm{T}(\mathrm{Ma})$ & MSWD & $\mathrm{N}$ \\
\hline \multicolumn{9}{|l|}{ GCB1 } \\
\hline GCL7 & $1402 \pm 32$ & $18012 \pm 96$ & $49401 \pm 207$ & $297 \pm 7$ & 19 & $303 \pm 9$ & 9 & 31 \\
\hline GEB2 & $1791 \pm 38$ & $18590 \pm 80$ & $64369 \pm 206$ & $310 \pm 17$ & 12 & & & \\
\hline \multicolumn{9}{|l|}{ GCB2 } \\
\hline $\mathrm{GIN}$ & $666 \pm 28$ & $6205 \pm 73$ & $28751 \pm 195$ & $304 \pm 15$ & 7 & $301 \pm 4$ & 2 & 38 \\
\hline GIN2 & $948 \pm 19$ & $7028 \pm 33$ & $48451 \pm 94$ & $297 \pm 6$ & 31 & & & \\
\hline \multicolumn{9}{|l|}{ GCB3 } \\
\hline REPB & $1043 \pm 20$ & $6566 \pm 39$ & $5595 \pm 112$ & $301 \pm 4$ & 32 & $301 \pm 4$ & 3 & 32 \\
\hline \multicolumn{9}{|l|}{ GCB4 } \\
\hline GM & $768+31$ & $2740+74$ & $48670+262$ & $297+14$ & 16 & $297+3$ & 35 & 45 \\
\hline NAC & $1231 \pm 23$ & $7439 \pm 31$ & $67602 \pm 90$ & $300 \pm 6$ & 29 & & & \\
\hline \multicolumn{9}{|l|}{ GCB5 } \\
\hline LARDO & $1285+22$ & $13178+52$ & $50581+85$ & $301+3$ & 49 & $301+3$ & 14 & 49 \\
\hline
\end{tabular}

GCB1, GCB2, GCB3, GCB4 and GCB5 are those in Figure 1. N - Number of analysis.

Table 1. EPMA U-Th-Pb monazite data from granitic rocks of the Castelo Branco pluton

The obtained results of monazite from the granite GCB4 reveal heterogeneity, supported by the higher MSWD values observed which may be associated with the uncertainties of this methodology [44]. However, they may also be related to the occurrence of geological processes responsible for the presence of some initial $\mathrm{Pb}$ in monazite grains or alteration processes.

The U-Th- $\mathrm{Pb}$ monazite age obtained by electron microprobe is an important alternative geochronological method, which allows to obtain accurate and similar results to those obtained by isotopic dating ages [8]. The potentiality of this geochronological methodology increases 
with the application togheter with other isotopic dating methods, such as $\mathrm{U}-\mathrm{Pb}$ zircon and monazite [7].

The monazite ages obtained through U-Th- $\mathrm{Pb}$ (EPMA) do not allow themselves to evaluate the accuracy of obtained ages because there is concordance between $\mathrm{Th}-\mathrm{Pb}$ and $\mathrm{U}-\mathrm{Pb}$ systems. However, the consistency of the obtained measurements allows to conclude that a crystal was not significantly altered or modified [7]. The most recent monazite age values obtained by EPMA may indicate an isotopic discordance leading to a more relatively recent age with a smaller ratio $\mathrm{Pb} /(\mathrm{Th}+\mathrm{U})$ [45]. U-Th- $\mathrm{Pb}$ (EPMA) monazite age correponds to the minimum ${ }^{207} \mathrm{~Pb} /$ ${ }^{235} \mathrm{U}$ age and maximum ${ }^{208} \mathrm{~Pb} /{ }^{232} \mathrm{Th}$ age. However, these isotopic disagreements are not possible to assess by electron microprobe [45].

Monazites from granites GCB1 and GCB5 and granodiorites GCB2 and GCB3 presented the ages of 301-303 Ma, whereas monazite from the granite GCB4 tends to be more recent (297 Ma; Table 1). However, the various ages are similar and within the range of the analytical error. The highest MSWD values on monazites from granodiorite GCB4 and granite GCB5 must be associated with a disperion of the results, which could be related to the methodology uncertainty or to a possible geological or alteration processes.

\section{2. $\mathrm{U}-\mathrm{Pb}$ zircon and monazite ages}

$\mathrm{U}-\mathrm{Pb}$ isotopic analyses were carried out on zircon and monazite from representative samples of the granitic rocks GCB1, GCB2 and GCB5 from the Castelo Branco pluton using the ID-TIMS method [25].

Granodiorite GCB1 contains hyaline or colorless zircons, euhedral elongated prismatic and subhedral crystals with varied size and forms. Some of them contain associated fractures and rare inclusions (Fig. 3a).

Monazite crystals (Fig. 3b) are reversely discordant, which can be associated with Paleozoic geological processes and ${ }^{207} \mathrm{~Pb} /{ }^{235} \mathrm{U}$ concordant monazite age should be used (Table 2). The reversly concordia can be interpreted as the result of the existence of some inherited $\mathrm{Pb}$, associated with an excess of initial ${ }^{230} \mathrm{Th}$ [46-47] due to possible inherited zircon cores. For other authors, it could be justified with some degree of change [36] or incomplete dissolution [37]. Some zircons have inherited cores and were not considered for the U-Pb age. The age ${ }^{207} \mathrm{~Pb} /$ ${ }^{206} \mathrm{~Pb}$ of $309.9 \pm 1.0 \mathrm{Ma}$ was obtained for a more concordant zircon crystal and is similar to the zircon concordia age 309.9 $\pm 1.1 \mathrm{Ma}$ (Fig. 4; Table 2). Monazite from granite GCB1 plots slightly reversely discordant (Fig. 4), a fact commonly linked to ${ }^{230} \mathrm{Th}$ initial excess, which eventually results in an excess of ${ }^{206} \mathrm{~Pb}$ and reverse discordance [46-47]. The ${ }^{207} \mathrm{~Pb} /{ }^{235} \mathrm{U}$ ratio is not affected by this disequilibrium effect and can be used as the closest estimate for monazite age. The concordant monazite of granite GCB1 yields a ${ }^{207} \mathrm{~Pb} /{ }^{235} \mathrm{U}$ age $309.5 \pm 0.9 \mathrm{Ma}$, which overlaps the zircon crystal of the granite GCB1 (Fig. 4; Table 2).

Zircons from granodiorite GCB2 occur as hyaline to slightly pinkish, elongated prismatic crystals or subhedral crystals with longitudinal cracks and occasional inclusions (Fig. 3c). Monazite is euhedral with rare inclusions (Fig. 3d). Zircon crystals from the granodiorite GCB2 plot near the concordia, but one of them presents a restitic core and deviates from the curve 

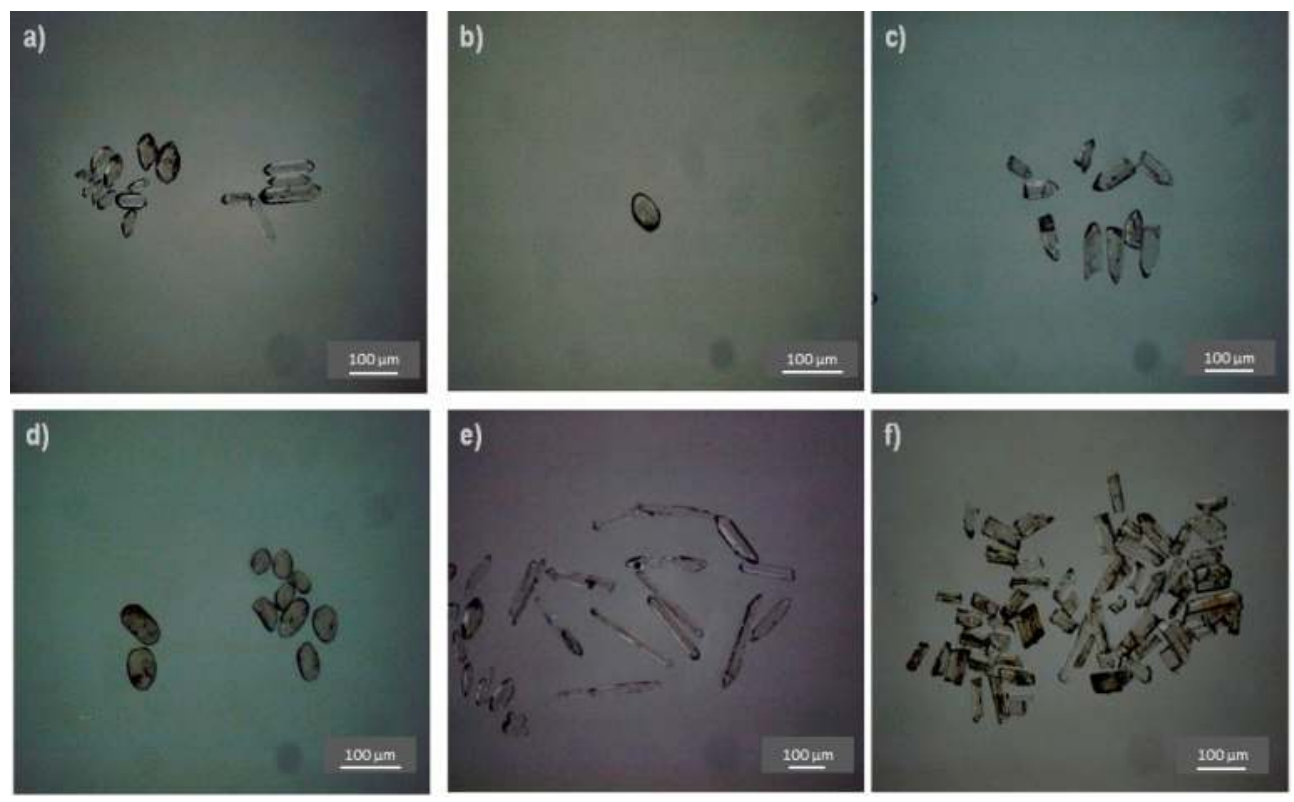

Figure 3. Selected zircon and monazite crystals from granitic rocks of Castelo Branco pluton. Muscovite $>$ biotite granite GCB1: zircon (a), monazite (b); biotite > muscovite granodiorite GCB2: zircon (c) monazite (d); muscovite > biotite granite GCB5: zircon $(e, f)$.

(Fig. 4). So, the concordia age was not considered. The concordant zircon yields a ${ }^{207} \mathrm{~Pb} /{ }^{206} \mathrm{~Pb}$ age of $310.1 \pm 0.8 \mathrm{Ma}$ (Fig. 4; Table 2). The projection of monazite fraction is slightly above the concordia curve and gives an age of $310.6 \pm 1.5 \mathrm{Ma}$, obtained by ${ }^{207} \mathrm{~Pb} /{ }^{235} \mathrm{U}$ ratio (Fig. 4; Table 2). The age of $310.1 \pm 0.8 \mathrm{Ma}$ obtained from the concordant zircon crystal should be considered for the granodiorite GCB2 Table 2 .

\begin{tabular}{llll}
\hline & GCB1 & GCB2 & GCB5 \\
\hline Zircon concordia age & $309.9 \pm 1.1 \mathrm{Ma}$ & - & $309.1 \pm 0.6$ \\
& MSWD $=1.11$ & & MSWD $=0.11$ \\
\hline${ }^{207} \mathrm{~Pb} /{ }^{206} \mathrm{~Pb}$ concordant zircon age & $309.9 \pm 1.0 \mathrm{Ma}$ & $310.1 \pm 0.8 \mathrm{Ma}$ & $309.7 \pm 0.4 \mathrm{Ma}$ \\
\hline${ }^{207} \mathrm{~Pb} /{ }^{235} \mathrm{U}$ concordant monazite age & $309.5 \pm 0.9 \mathrm{Ma}$ & $310.6 \pm 1.5 \mathrm{Ma}$ & $309.7 \pm 0.4 \mathrm{Ma}$ \\
\hline
\end{tabular}

GCB1, GCB2 and GCB5 as in Figure 1.

Table 2. U-Pb zircon and monazite ages of granitic rocks from the Castelo Branco pluton

Granite GCB5 contains hyaline and colorless elongated prismatic zircons and several subhedral crystals and some slightly rounded, corresponding to fractions or fragmented crystals, 

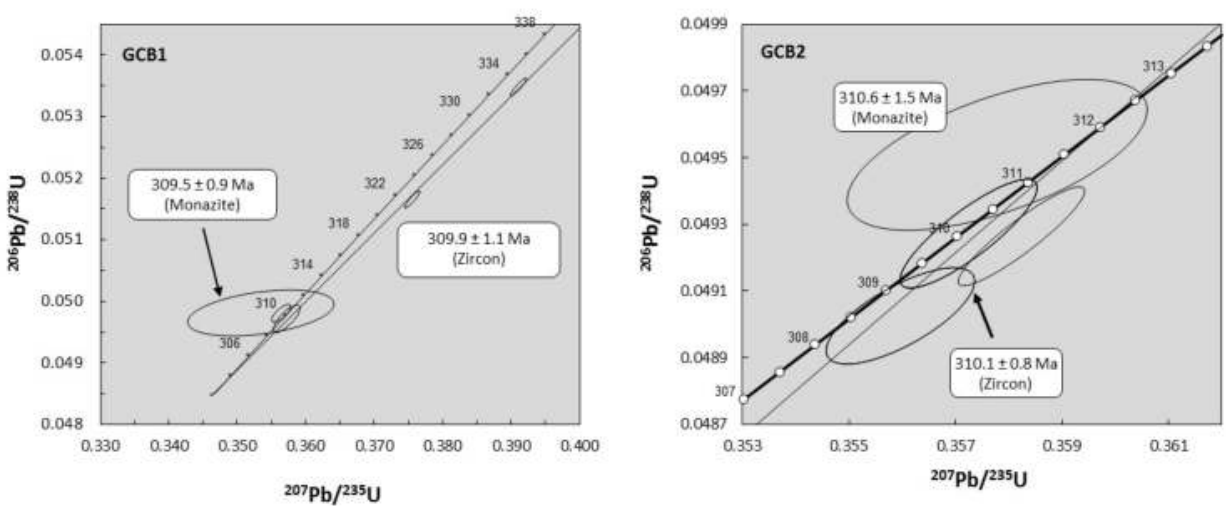

Figure 4. Selected concordia ${ }^{207} \mathrm{~Pb} /{ }^{235} \mathrm{U}$ versus ${ }^{206} \mathrm{~Pb} / 238 \mathrm{U}$ diagrams for zircon and monazite of muscovite $>$ biotite granite GCB1 and biotite > muscovite granodiorite GCB2 from the Castelo Branco pluton (Error for ellipses are drawn at $2 \sigma)$.

some brownish colourless with inclusions (Fig. 3e; 3f). Monazite is unsual and with rare inclusions. The zircon crystals define a discordia line yielding a lower intersect age of $309.1 \pm$ $0.6 \mathrm{Ma}(\mathrm{MSWD}=0.11)$, similar to the ${ }^{207} \mathrm{~Pb} /{ }^{206} \mathrm{~Pb}$ concordant zircon age $(309.7 \pm 0.4 \mathrm{Ma})$, and an upper intersect age of about $1000 \mathrm{Ma}$. Concordant monazite has a ${ }^{207} \mathrm{~Pb} / 235 \mathrm{U}$ similar age of 309.7 $\pm 0.4 \mathrm{Ma}$ (Table 2).

The average $\mathrm{U}-\mathrm{Pb}$ age obtained for each granite from the Castelo Branco pluton is similar (310 $\mathrm{Ma}$ ), indicating that they are contemporaneous (Table 2).

Zircon from granite GCB5 is the richest in U content (GCB5 511 ppm) of granitic rocks from Castelo Branco pluton (GCB1 384 ppm; GCB2 411 ppm) and with the highest ${ }^{206} \mathrm{~Pb} /{ }^{204} \mathrm{~Pb}$ ratio (GCB1 7932; GCB2 4571; GCB5 23130). Monazite from GCB5 also presents the highest ${ }^{206 \mathrm{~Pb} /}$ ${ }^{204} \mathrm{~Pb}$ ratio (GCB1 1281; GCB2 1340; GCB5 7409) [25]. Zircon Th/U ratio from granitic rocks of Castelo Branco pluton ranges between $0.10-0.88$, corresponding to zircons with an igneous signature [48]. Zircon from granodiorite GCB2 presents the highest Th/U variation range (Th/ $\mathrm{U}$ 0.18-0.88) including the values obtained from granodiorite GCB1 zircon (Th/U 0.10-0.28) and granite GCB5 (Th/U 0.11-0.14). The selected monazite crystals from granite GCB1 show the highest variability $U$ and $T h / U$ results including the highest and the lowest value of $U$ (U 159 -4514 ppm) and Th/U ratio (4.58 - 109.34) from the granitic rocks of the Castelo Branco pluton.

The discordant monazites tend to have higher levels of Th, identified by the highest values of $\mathrm{Th} / \mathrm{U}$ ratio [36]. However, although high Th values may contribute to the discrepancy associated with monazite crystals is not possible to establish a linear correlation between $\mathrm{Th} / \mathrm{U}$ ratio and the degree of monazites discordance. In some monazite crystals, $\mathrm{U}-\mathrm{Pb}$ ratio and presented features could have a greater influence on the associated discordance [36]. The discordance of monazite crystals from granite CBG5 could be associated with ${ }^{230} \mathrm{Th}$ disequilibrium and U loss, which can be justified by possible crystal change, as found in monazites from Suomujärvi 
Complex [36]. In monazite crystals, there are considerable amounts of $\mathrm{Pb}$ and $\mathrm{Th}$ that could be incorporated and the enrichment or depletion of $U$ will depend on fluid composition and associated processes [49].

The $\mathrm{U}-\mathrm{Pb}$ monazite and $\mathrm{U}-\mathrm{Th}-\mathrm{Pb}$ isotopic data confirm that the Castelo Branco pluton consists of five concentric late-tectonic Variscan granitic bodies, which intruded the Cambrian schist greywacke.

\section{3. $\mathrm{Rb}-\mathrm{Sr}, \mathrm{Sm}-\mathrm{Nd}$ and $\delta^{18} \mathrm{O}$ whole rock}

For $\mathrm{Rb}-\mathrm{Sr}$ and $\mathrm{Sm}-\mathrm{Nd}$ isotopic studies, representative samples of the five granitic rocks from the Castelo Branco pluton were selected, after a detailed geological field study, petrographic and geochemical whole rock data interpretation. The $\mathrm{Rb} / \mathrm{Sr}$ isotopic age obtained for granitic rocks from Castelo Branco pluton ( $300 \pm 24 \mathrm{Ma}$; [50]) is lower than the U-Pb zircon and monazite ages (310 Ma; Table 2), which could be atributed to an opening of the Rb-Sr system during the metamorphic event associated with late-D3 granite intrusions, or furthermore to the lower temperature of $\mathrm{Rb}-\mathrm{Sr}$ system than $\mathrm{U}-\mathrm{Pb}$ system [23]. Initial $\left({ }^{87} \mathrm{Sr} /{ }^{86} \mathrm{Sr}\right)$ ratios and $\varepsilon \mathrm{Nd}_{\mathrm{T}}$ values were calculated using the U-Pb age of $310 \mathrm{Ma}$ (Table 3). Petrographic and geochemical characteristics of granodiorite GCB3 and granite GCB4 suggest that they are related to granodiorite GCB2, and a similar age of 310 Ma have been considered.

\begin{tabular}{lccccc}
\hline & GCB1 & GCB2 & GCB3 & GCB4 & GCB5 \\
\hline${ }^{\left.{ }^{87} \mathrm{Sr} / 86 \mathrm{Sr}\right)_{310}}$ & $0.7082-0.7098$ & $0.7085-0.7128$ & 0.7104 & $0.7078-0.7104$ & 0.7120 \\
\hline$\varepsilon \mathrm{Nd}_{310}$ & $-3.8 \pm 0.2$ & $-1.7 \pm 0.4$ & -0.8 & $-2.8 \pm 0.68$ & -3.0 \\
\hline $\mathrm{T}_{\mathrm{DM}}$ & $1.64 \pm 0.12$ & $1.10 \pm 0.0$ & 1.11 & $1.35 \pm 0.1$ & 1.56 \\
\hline$\delta^{18} \mathrm{O}(\% \mathrm{o})$ & $13.53 \pm 0.11$ & $12.27 \pm 0.04$ & 12.50 & 12.75 & $12.91 \pm 0.23$ \\
\hline
\end{tabular}

GCB1, GCB2, GCB3, GCB4 and GCB5 as in Figure 1.

Table 3. $\varepsilon \mathrm{Nd}_{310}$ and $\delta^{18} \mathrm{O}$ data of granitic rocks from the Castelo Branco pluton

The $\left({ }^{87} \mathrm{Sr} /{ }^{86} \mathrm{Sr}\right)_{310}$ ratios obtained for granite GCB1, granodiorite GCB2 and granite GCB5 are characteristic of granites resulting from crustal anatexis of metasedimentary rocks (Table 3).

The $\varepsilon \mathrm{Nd}_{310}$ and $\mathrm{T}_{\mathrm{DM}}$ values obtained for representative samples from granitic rocks of Castelo Branco pluton were calculated [51] with a ${ }^{147} \mathrm{Sm}$ desintegration constant of $\lambda=6.54{ }^{*} 10^{-12}$ year $^{-1}$ [52]. To $\varepsilon \mathrm{Nd}_{\mathrm{T}}$ and $\mathrm{T}_{\mathrm{DM}}$ calculation ${ }^{143} \mathrm{Nd} /{ }^{144} \mathrm{Nd}_{\mathrm{CHUR}}=0.512638$ and ${ }^{147} \mathrm{Sm} /{ }^{144} \mathrm{Nd}_{\mathrm{CHUR}}=0.1967$ [53] and ${ }^{143} \mathrm{Nd} /{ }^{144} \mathrm{Nd}_{\mathrm{DM}}=0.513151$ and ${ }^{147} \mathrm{Sm} /{ }^{144} \mathrm{Nd}_{\mathrm{DM}}=0.22$ [54] were applied, respectively.

Granites GCB1 and GCB5 show lower values of $\varepsilon \mathrm{Nd}_{310}$ and higher values of $\mathrm{T}_{\mathrm{DM}}$ than granodiorites GCB2, GCB3 and granite GCB4 (Table 3), suggesting that GCB1 and GCB5 granites are not relate to the granodiorites GCB2 and GCB3 and granite GCB4 from the Castelo Branco pluton. 
The diagram $\varepsilon \mathrm{Nd}_{310}$ versus $\left({ }^{87} \mathrm{Sr} /{ }^{86} \mathrm{Sr}\right)_{310}$ shows a dominantly crustal origin for all granitic rocks from the Castelo Branco pluton. All samples plot on the field IV, indicating that are derived from $\mathrm{Rb}$ enriched and depleted Sm protholiths (Fig. 5a). The scatter of $\left({ }^{87} \mathrm{Sr} /{ }^{86} \mathrm{Sr}\right)_{310}$ ratio suggests that the granitic rocks were not in complete isotopic equilibrium at the time of formation, which is also supported by an heterogeneous $\varepsilon \mathrm{Nd}_{310}$ (Fig. 5a).
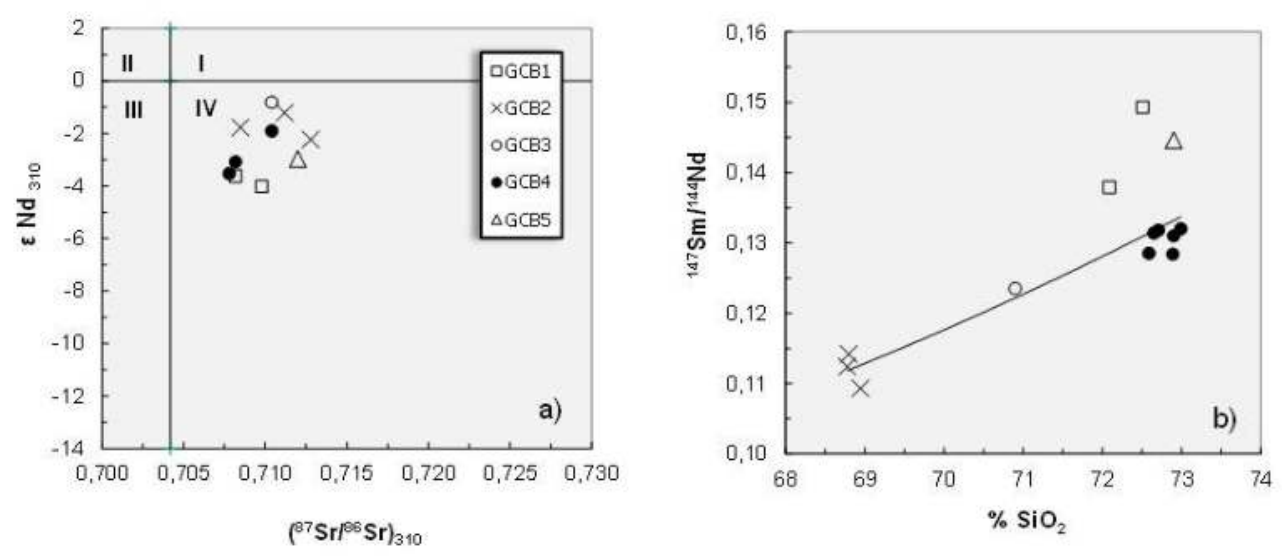

Figure 5. Selected diagrams of granitic rocks from Castelo Branco pluton: a) $\varepsilon \mathrm{Nd}_{310}$ versus $\left.\left({ }^{87} \mathrm{Sr} /{ }^{86} \mathrm{Sr}\right)_{310} ; \mathrm{b}\right){ }^{147} \mathrm{Sm} /{ }^{144} \mathrm{Nd}$ versus $\mathrm{SiO}_{2}$. GCB1, GCB2, GCB3, GCB4 and GCB5 as in Figure 1.

The obtained results show coherence within each of the five granitic rocks from the Castelo Branco pluton with a slightly evolutionary trend from higher to lower values of $\left({ }^{87} \mathrm{Sr} /{ }^{86} \mathrm{Sr}\right)_{310}$ and $\varepsilon \mathrm{Nd}_{310}$ from granodiorite GCB2 and GCB3 to granite GCB4 [25]. Granites GCB1 and GCB5 tend to have the most negative values for $\varepsilon \mathrm{Nd}_{310}$, with variable $\left({ }^{87} \mathrm{Sr} /{ }^{86} \mathrm{Sr}\right)_{310}$. These distributions suggest the contribution of at least three magmatic components GCB1, GCB2 and GCB5 with different isotopic signatures (Table 3; Fig. 5a). All the data plot within a field delimited by $\varepsilon \mathrm{Nd}=-1$ to -4 and ${ }^{87} \mathrm{Sr} /{ }^{86} \mathrm{Sr}=0.708-0.712$, which indicate derivation from crustal material with average Mesoproterozoic mantle extraction ages (Fig. 5a). $\mathrm{T}_{\mathrm{DM}}$ values of granitic rocks are similar to the $\mathrm{T}_{\mathrm{DM}}$ data for schist-greywacke complex from the studied area [55].

The ${ }^{147} \mathrm{Sm} /{ }^{144} \mathrm{Nd}$ versus $\mathrm{SiO}_{2}$ diagram of granitic rocks from the Castelo Branco pluton shows a positive correlation with an increase of ${ }^{147} \mathrm{Sm} /{ }^{144} \mathrm{Nd}$ from granodiorite GCB2, to granodiorite GCB3 and granite GCB4. Otherwise, granites GCB1 and GCB5 do not present similar variation and plot outside the straight line (Fig. 5b).

Whole rock oxygen-isotope $\left(\delta^{18} \mathrm{O}\right)$ values, obtained for eight representative samples of granitic rocks from the Castelo Branco pluton, range from +12.27 to $+13.53 \%$ (Table 3 ). The obtained $\delta^{18} \mathrm{O}$ values are higher than $10 \%$, indicating that the granitic rocks from the Castelo Branco pluton correspond to S-type granites [56].

There is a progressive increase of $\delta^{18} \mathrm{O}$ from granodiorite GCB2 to granodiorite GCB3, granite GCB4 and granite GCB5. The $\delta^{18} \mathrm{O}$ values are also positively correlated with $\mathrm{SiO}_{2}$, 
$\mathrm{Li}, \mathrm{Rb}$ and negatively correlated with $\mathrm{FeO}, \mathrm{Sr}$ and $\mathrm{Ba}$ [25]. In these diagrams, granodiorite GCB2 and GCB3 and granite GCB4 define a curvilinear variation trend which is characteristic of a magmatic differentiation process. Granite GCB1 yields the highest value, which deviates clearly from the trend, whereas granite GCB5 plots closer, but in average has higher $\delta^{18} \mathrm{O}$ than the trend [25].

A system without contamination will present radiogenic isotopic characteristics similar to the original source, even with the occurence of magmatic differentiation processes [17]. However, the values of $\delta^{18} \mathrm{O}$ show small variations in the magmatic differentiation processes, with an increase of about 1 to $1.2 \%$, increasing with the degree of differentiation [18-24]. The $\delta^{18} \mathrm{O}$ values increase from the granodiorite GCB2 $(12.27 \%$ o) to the granodiorite GCB4 $(12.75 \%$ ), which is associated with the fractional crystallization process [24].

These isotopic ratios $\left({ }^{87} \mathrm{Sr} /{ }^{86} \mathrm{Sr}\right)_{310}, \varepsilon \mathrm{Nd}_{310}$ and $\delta^{18} \mathrm{O}$ values of granite GCB1, granodiorite GCB2 and granite GCB5 are of granites originated from crustal anatexis of metasedimentary rocks [25].

\section{Conclusions}

$\mathrm{U}-\mathrm{Th}-\mathrm{Pb}$ monazite ages from granodiorite GCB1 and granite GCB5 and granodiorites GCB2 and GCB3 have a similar values, ranging from 303-301 Ma. Granodiorite GCB4 presents a lower value of $297 \pm 3 \mathrm{Ma}$, but the difference is insided the analytical error. However, U-Th- $\mathrm{Pb}$ monazite ages for granodiorites GCB1 and GCB2 and granite GCB5 are consistently below about $7 \mathrm{MA}$, with respect to the age of $310 \pm 1 \mathrm{Ma}$ obtained by ID-TIMS U-Pb zircon and monazite.

The most recent ages obtained by U-Th-Pb monazite EPMA age could be associated to the higher levels of $U$ and $T h$ and lower $T h / U$ ratio obtained by electron microprobe relatively to those obtained by ID-TIMS, as well as $\mathrm{Pb}$ partial loss like as found in rocks of southern India [1]. The U-Pb zircon and monazite isotopic ages (ID-TIMS) are the most accurate. However, $\mathrm{U}-\mathrm{Th}-\mathrm{Pb}$ monazite ages (EPMA) are closer.

The granitic rocks GCB1, GCB2 and GCB5 correspond to three distinct magmatic pulses and have a similar age of $310 \pm 1 \mathrm{Ma}$ (ID-TIMS U-Pb zircon and monazite). These three granitic rocks have different $\left({ }^{87} \mathrm{Sr} /{ }^{86} \mathrm{Sr}\right)_{310}, \varepsilon \mathrm{Nd}_{310}$ and $\delta^{18} \mathrm{O}$ values and correspond to three distint magmatic pulses derived by partial melting of heterogeneous metasedimentary materials.

\section{Acknowledgements}

Funding was provided to I.M.H.R. Antunes by the SFRH/BD/2885/2000 grant from the Fundação para a Ciência e a Tecnologia, Portugal. 


\section{Author details}

Antunes Imhr ${ }^{1,2,3}$

Address all correspondence to: imantunes@ipcb.pt

1 Polytechnic Institute of Castelo Branco and Geo-Environmental and Resources Research Center, Portugal

2 Neiva AMR, Department of Earth Sciences and Geosciences Centre, University of Coimbra, Portugal

3 Silva MMVG, Department of Earth Sciences and Geosciences Centre, University of Coimbra, Portugal

\section{References}

[1] Braun I, Montel JM, Nicollet C. 1998. Electron microprobe dating of monazites from high-grade gneisses and pegmatites of the Kerala Khondalite Belt, southern India. Chem. Geol. 146, 65-85.

[2] Orejana D, Merino E, Villaseca C, Pérez-Soba C, Cuesta A. 2012. Electron microprobe monazite geochronology of granitic intrusions from the Montes de Toledo batholith (central Spain). Geol. J. 47, 41-58.

[3] Meldrum A, Boatner LA, Weber WJ, Ewing RC. 1998. Radiation damage in zircon and monazite. Geochim. Cosmich. Acta 62, 2509-2520.

[4] Cherniak DJ, Watson EB, Grove M, Harrison TM. 2004. Pb diffusion in monazite: a combined RBS/SIMS study. Geochim. Cosmochim. Acta 68, 829-840.

[5] Suzuki K, Adachi M. 1994. Middle precambrian detrital monazite and zircon from the Hida gneiss on Oki-Dogo Island, Japan: their origin and implications for the correlation of basement gneiss of southwest Japan and Korea. Tectonophysics 235, 277-292.

[6] Montel JM, Foret S, Veschambre M, Nicollet C, Provost A. 1996. Electron microprobe dating of monazite. Chem. Geol. 131, 37-53.

[7] Williams ML, Jercinovic MJ. 2002. Microprobe monazite geochronology: putting absolute time into microstructural analysis. J. Struct. Geol. 24, 1013-1028.

[8] Cocherie A, Mezeme EB, Legendre O, Fanning CM, Faure M, Rossi P. 2005. Electronmicroprobe dating as a tool for determining the closure of Th-U-Pb systems in migmatitic monazites. Am. Mineral. 90, 607-618. 
[9] Harrison TM, McKeegan KD, Le Fort P. 1995. Detection of inherited monazite in the Manaslu leucogranite by ${ }^{208} \mathrm{~Pb} /{ }^{232} \mathrm{Th}$ ion microprobe dating: crystallization age and tectonic implications. Earth Plant. Sci. Lett. 133, 271-282.

[10] Cocherie A, Legendre O, Pecaut JJ, Kouamelan AN. 1998. Geochronology of polygenetic monazites constrained by in situ electron microprobe Th-U-total lead determination: implications for lead behaviour in monazite. Geochim. Cosmochim. Acta 62, 2475-2497.

[11] Be Mezene E, Cocherie A, Faure M, Legendre O, Rossi P. 2006. Electron microprobe monazite geochronology of magmatic events examples from Variscan migmatites and granitoids, Massif Central, France. Lithos 87, 276-288.

[12] Kohn MJ, Malloy MA. 2004. Formation of monazite via prograde metamorphic reactions among common silicates: implications for age determinations. Geochim. Cosmochim. Acta 68, 101-113.

[13] Kelsey DE, Clark C, Hand M. 2008. Thermobarometric modeling of zircon and monazite growth in melt-bearing systems: examples using model metapelitic and metapsammitic granulites. J. Metam. Geol. 26, 199-212.

[14] Corfu F, Hanchar JM, Hoskin PWO, Kinny P. 2003. Atlas of zircon textures. In: Hanchar JM, Hoskin PWO (Eds). Zircon, Rev. Miner. Geochem. 53, 469-500.

[15] Ayres JD, Loflin M, Miller CF, Barton MD, Coath CD. 2006. In site oxygen isotope analysis of monazite as monitor of fluid infiltration during contact metamorphism: Birch Creek Pluton aureole, White Mountains, Eastern California. Geol. 34, 553-656.

[16] Harlov DE, Wirth R, Hetherington CJ. 2007. The relative stability of monazite and huttonite at $300-900^{\circ} \mathrm{C}$ and $200-1000 \mathrm{MPa}$ : metasomatism and the propagation of metastable mineral phases. Am. Mineral. 92, 1652-1664.

[17] Rollinson H. 1993. Using Geochemical Data: Evalution, Presentation and Interpretation. Longman, UK. 352 pp.

[18] Taylor Jr HP, Epstein S. 1962. Relationships between ${ }^{18} \mathrm{O} /{ }^{16} \mathrm{O}$ ratios in coexisting minerals of magmatic and metamorphic rocks: Part I. Principles and experimental results. Geol. Soc. Amer. Bull. 73, 461-480.

[19] Epstein S, Taylor HP Jr. 1972. ${ }^{18} \mathrm{O} /{ }^{16} \mathrm{O},{ }^{30} \mathrm{Si} /{ }^{28} \mathrm{Si},{ }^{13} \mathrm{C} /{ }^{12} \mathrm{C}$ and $\mathrm{D} / \mathrm{H}$ studies of Apollo 14 and 15 samples, Proc. $3^{\text {rd }}$ Lunar Sci. Conf. Geochim. Cosmochim. Acta, Suppl. 3/2, 1429.

[20] Muehlenbachs K, Byerly G. 1982. ${ }^{18} \mathrm{O}$-Enrichment of silicic magmas caused by crystal fractionation at the Galapagos Spreading Center. Contrib. Mineral. Petrol. 79, 76-79.

[21] Kalamarides RI. 1984. Kiglapait geochemistry VI: oxygen isotopes. Geochim. Cosmochim. Acta 46, 1863-1868. 
[22] Sheppard MF, Harris C. 1985. Hydrogen and oxygen isotope geochemistry of ascension Island lavas and granites: variation with crystal fractionation and interaction with sea water. Contrib. Mineral. Petrol. 91, 74-81.

[23] Faure G. 1986. Principles of isotope geology. John Willey \& Sons, New York. 589 pp.

[24] White 2003. High temperature applications II: oxygen isotopes as an indicator of assimilation. Geol. 656 Isotope Geochemistry. Lectures 30, 227-231.

[25] Antunes IMHR, Neiva AMR, Silva MMVG, Corfu F. 2008. Geochemical and isotopic data on the granitic-granodioritic, concentrically zoned Castelo Branco pluton (central Portugal). Lithos 103 (3/4), 445-465.

[26] Antunes IMHR, Neiva AMR, Silva MMVG, Corfu F. 2009. The genesis of I-and Stype granitoid rocks of the Early Ordovician Oledo pluton, Central Iberian Zone (central Portugal). Lithos 111 (3/4), 168-185.

[27] Ancey M, Bastenaire F, Tixier R. 1978. Application des méthodes statistiques in microanalyse. In: Maurice F, Menyard L, Tixier R. (Eds.), Microanalyse-Microscope Électronique à Balayage Orsay: Les éditions des Physicien, 323-347.

[28] Montel JM, Veschambre M, Nicollet C. 1994. Datation de la monazite à la microsonde electronique (Dating monazite using electron microprobe). Comp. Rend. L'Académie Sci. 318, 1489-1495.

[29] Cocherie A, Albarède F. 2001. An improved U-Th-Pb age calculation for electron microprobe dating of monazite. Geochim. Cosmochim. Acta 65, 4509-4522.

[30] Krogh TE. 1982. Improved accuracy of U-Pb zircon ages by creation of more concordant systems using an air abrasion technique. Geochim. Cosmochim. Acta 46, 637-649.

[31] Tucker RD, Raheim A, Krogh TE, Corfu F. 1987. Uranium-lead zircon and titanite ages from the northern portion of the Western Gneiss Region, south-central Norway. Earth Planet. Sci. Lett. 81, 203-211.

[32] Davidson A, Van Breemen O. 1988. Baddedeleyite-zircon relationships in coronitic metagrabro, Grenville Province, Ontario: Implications for geochronology. Contrib. Mineral. Petrol. 100, 291-299.

[33] Davis DW, Blackburn CE, Krogh TE. 1982. Zircon U-Pb ages from the Wabigoon, Manitou Lakes Region, Wabigoon Subprovince, northwest Ontario. Can. J. Earth Sci. 19, 254-266.

[34] Krogh TE. 1973. A low contamination method for hydrothermal decomposition of zircon and extraction of $\mathrm{U}$ and $\mathrm{Pb}$ for isotopic age determination. Geochim. Cosmochim. Acta 37, 485-494. 
[35] Corfu F, Andersen TB. 2002. U-Pb ages of the Dalsfjord Campus, SW-Norway, and their bearing on the correlation of the allochthonous crystalline segments of the Scandinavian Caledonides. Int. J. Earth Sci. 91, 955-963.

[36] Corfu F, Evins PM. 2002. Late Paleoproterozoic monazite and titanite U-Pb ages in the Archean Suomujäri complex, N Finland. Prec. Res. 116, 171-181.

[37] Corfu F. 2004. U-Pb geochronology of the Lekres Group: an exotic Early Caledonian metasedimentary assemblage stranded on Lofoten basement, northern Norway. J. Geol. Soc. (Lond.) 161, 619-627.

[38] Stacey JS, Kramers JD. 1975. Approximation of terrestrial lead isotope evolution by a two-stage model. Earth Planet. Sci. Lett. 34, 207-226.

[39] Jaffey AH, FlynnKF, Glendenin LE, Benthey WC, Essling AM. 1971. Precision measurement of half lives and specific activities of ${ }^{235} \mathrm{U}$ and ${ }^{238} \mathrm{U}$. Phys. Rev., C Nucl. Phys. 4, 1889-1906.

[40] Ludwig KR. 1999. Isoplot/Ex version 2.03. A geochronological toolkit for Microsoft Excel. Berkeley Geochronology under Special Publication, vol. 143 pp.

[41] Montero P, Bea F. 1998. Accurate determination of ${ }^{87} \mathrm{Rb} /{ }^{86} \mathrm{Sr}$ and ${ }^{147} \mathrm{Sm} /{ }^{144} \mathrm{Nd}$ by inductively coupled plasma mass spectrometry in isotope geoscience: an alternative to isotope dilution analysis. Anal. Chim. Acta 358, 22-233.

[42] Govindaraju K, Potts PJ, Webb PC, Watson JS. 1994. Report on Whin Sill Dolerite WE from England and Pitscurrie Microgabbro PM-S from Scotland: assessment by one hundred and four international laboratories. Geostand. Newslett. 18, 211-300.

[43] York D. 1969. Least-squares fitting of a straight line with correlated error. Earth Planet. Sci. Lett. 5, 320-324.

[44] Pyle JM, Spear FS, Wark DA, Daniel CG, Storm LC. 2005. Contributions to precision and accuracy of monazite microprobe ages. Am. Mineral. 90, 547-577.

[45] Kuiper YD. 2005. Isotopic age constraints from electron microprobe U-Th-Pb dates using a three-dimensional concordia diagram. Am. Mineral. 90, 586-591.

[46] Schärer U. 1984. The effect of initial ${ }^{230} \mathrm{Th}$ disequilibrium on young U-Pb ages: the Makalu case, Himalaya. Earth Planet. Sci. Lett. 67, 191-204.

[47] Kalt A, Corfu F, Wijbrans JR. 2000. Time calibration of a P-T path from a Variscan high-temperature low-pressure metamorphic complex (Bayerische Wald, Germany) and the detection of inherited monazite. Contrib. Mineral. Petrol. 138, 143-163.

[48] Williams IS, Claesson S. 1987. Isotopic evidence for the Precambrian provenance and Caledonian metamorphism of high grade paragneisses from the Seve Nappes, Scandinavian Caledonides. II. Ion microprobe zircon U-Th-Pb. Contrib. Mineral. Petrol. $97,205-217$. 
[49] Poitrasson F, Chenery S, Bland DJ. 1996. Contrasted monazite hydrothermal alteration mechanisms and their geochemical implications. Earth Planet. Sci. Lett. 145, 79-96.

[50] Antunes IMHR, 2006. Mineralogia, Geoquímica e Petrologia de rochas granitóides da área de Castelo Branco-Idanha-a-Nova. Unpublished PhD. thesis. University of Coimbra, Portugal, 453 pp.

[51] De Paolo DJ. 1981. Trace elements and isotopic effects of combined wall rock assimilation and fractional crystallization. Earth Planet. Sci. Lett. 53, 189-202.

[52] Steiger RH, Jäger E. 1977. Convention of the use of decay constants in geo and cosmochronology. Earth Planet. Sci. Lett. 36, 359-362.

[53] Jacobsen SB, Wasserburg GJ. 1980. Sm-Nd isotopic evolution of chondrites and achondrites. Earth Planet. Sci. Lett. 67, 137-150.

[54] Liew TC, Hofmann AW. 1988. Precambrian crustal components, plutonic associations, plate environment of the Hercynian Fold Belt of Central Europe: indications from a Nd and Sr isotopic study. Contrib. Mineral. Petrol. 98, 129-138.

[55] Beetsma JJ. 1995. The late Proterozoic/Paleozoic and Hercynian crustal evolution of the Iberian Massif, N Portugal. Unpublished PhD. thesis. Vrije Universiteit Amsterdam, 223 pp.

[56] Chappell BW, White AJR. 1992. I-and S-type granites in the Lachlan Fold Belt. Trans. R. Soc. Edinb. Earth Sci. 83, 1-26.
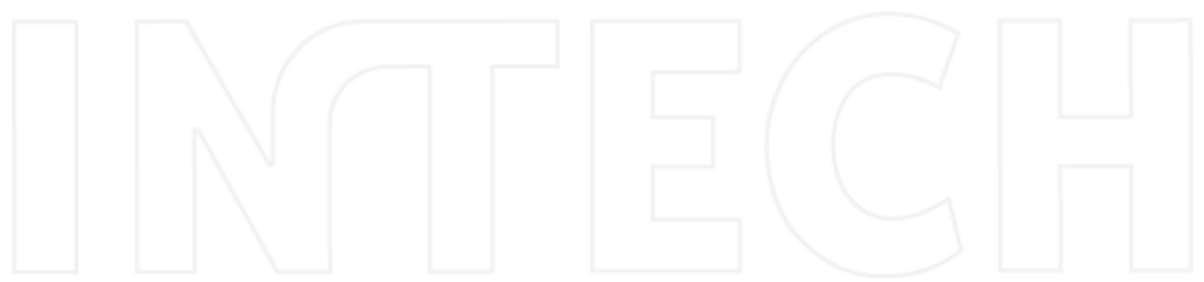
Chapter 5

\title{
In situ U-Pb Dating Combined with SEM Imaging on Zircon - An Analytical Bond for Effective Geological Recontructions
}

\author{
Annamaria Fornelli, Giuseppe Piccarreta and \\ Francesca Micheletti \\ Additional information is available at the end of the chapter \\ http://dx.doi.org/10.5772/58540
}

\section{Introduction}

In situ U-Pb dating combined with SEM images on zircon crystals represent a powerful tool to reconstruct metamorphic and magmatic evolution of basements recording a long and complex geological history [1-3].

The development of high spatial and mass resolution microprobes (e.g., LA-ICP-MS, SIMS, SHRIMP) allows in-situ measurements of $\mathrm{U}-\mathrm{Pb}$ ages in micro domains smaller than 30-50 microns $[4,5]$.

The growth of zircon crystals, evidenced by their internal microtextures, can be easily revealed by SEM imaging by Cathodoluminescence (CL) and Variable Pressure Secondary Electrons (VPSE) detectors on separated grains or in situ within a polished thin rock section [6,4,7].

Therefore it is possible to date different domains of single crystals, which may record magmatic or metamorphic events of the rock's geological history [8,4]. In acidic magmatic rocks abundant zircon crystals provide precise age data about magma emplacement and origin of source indicating the geodynamic context and the pertinence of terranes forming the continental crust.

As regards the metamorphic context, zircon can potentially preserves multiple stages of metamorphic records owing its highly refractory nature, high closure temperature and slow diffusion rate of $\mathrm{Pb}$, thus it is an ideal mineral for $\mathrm{U}-\mathrm{Pb}$ dating of poly-metamorphic rocks $[9,10]$. In addition, in situ analyses of trace elements such as rare earth elements (REE) in zircon and between zircon and coexisting minerals is usefull to decipher the REE behavior and 
mineral chemistry during metamorphism and to determine metamorphic P-T conditions $[8,11,12]$. In particular, garnet is one of the most important rock-forming minerals in high-grade metamorphic rocks since it can be also used to constrain metamorphic conditions if its composition is combined with that of other major minerals such as pyroxene and amphibole $[13,14]$. Relatively to REE partition in metamorphic rocks garnet, pyroxene, amphibole and zircon being competitors for REE partition, represent a usefull tool to outline continental crust evolution.

In this paper we present the geochronological and chemistry data collected in the last ten years in Calabria and Peloritani sectors of Italy, utilizing the new analytical techniques, usefull to reconstruct the magmatic and metamorphic history of a key sector of the South European Variscan Belt in the peri-Mediterranean area.

Metaigneous and metasedimentary rocks of the Calabria-Peloritani Terrane (Southern Italy) represent a particularity in the South Mediterranean area being connected to Alpine chain (Norther Italy) through sedimentary Apennines Chain. They rapresent sectors of Variscan upper, intermediate and lower continental crust sutured by a thick layer of CarboniferousPermian granitoids overlapped on Alpine oceanic crust units. Only rocks forming intermediate and deep crust levels of the continental crust were considered in this review. These rock types preserve memory of Precambrian to Permian geological events and in some cases up to Mesozoic times. The available geochronological data [15-24] togheter with CL and VPSE imaging and the REE-U-Th distribution in the zircon domains helped to depict the geological history through: (1) the emplacement ages of the protoliths of metaigneous rocks, (2) the contribution of the Neoproterozoic-Early Cambrian anatectic melts to produce the protoliths of Variscan metaigneous rocks, (3) the sedimentation ages of the protoliths of the metasedimentary rocks; (5) the P-T-t path of the Variscan metamorphism. In the following, an extensive and detailed description of utilized analytical techniques was presented together with the realized geological deductions relatively to Calabria-Peloritani Terrane.

\section{Analytical techniques}

\subsection{Sample preparation}

The U-Pb age data were obtained on zircons directly separated from samples or on polished thin rock sections. In the last case, in situ analyses allow to evaluate the micro-domains in which the zircon grew or was resetted. Separated zircon crystals were selected from 50-125 $\mu \mathrm{m}$ and 125-250 $\mu \mathrm{m}$ fractions extracted from about 5-10 kg of each rock sample. Crushing, heavy liquids, a Carpco and a Frantz magnetic separators have been used for mineral separation. The clearest, crack-and inclusion-free zircon grains were handpicked under binocular microscope and finally mounted in epoxy resin (Fig.1).

Only in the case of SIMS analyses, selected crystals were mounted together with chips of zircon standards (Fig.1), whereas for LA-ICP-MS analyses external standards were used. Grains were 


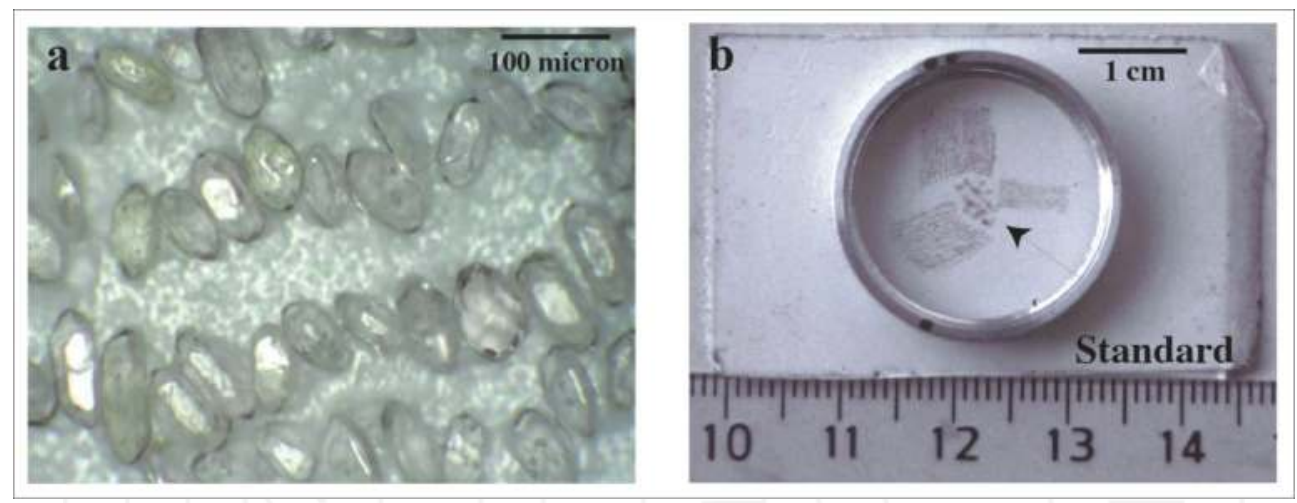

Figure 1. Zircon crystals separated from a rock sample (a) and placed in a mount with standard crystals (b).

then polished to half their thickness to expose internal structures. In the case of selected zircons in thin section, they are chosen also for their peculiar structural site.

\subsection{Zircon imaging}

All crystals were inspected under transmitted light and by SEM (Scanning Electron Microscope) in order to investigate their morphology and collect high-resolution images unravelling the internal microstructures. The first observations on zircon crystals were realized by BSED (Back-Scattered Electron Detector), in order to examine morphologic characters and the possible presence of inclusions of other minerals.

The high-resolution images of internal zoning patterns of zircons were realized by CL (Cathodoluminescence) and by VPSE (Variable Pressure Secondary Electrons) detectors, the last used in high vacuum conditions. Operating conditions were an accelerating voltage of $15 \mathrm{kV}$ with a beam current of $20 \mathrm{nA}$ for CL images and $100 \mathrm{nA}$ for VPSE images. The images obtained by two different detectors (CL and VPSE, Fig.2) are almost completely overlapping $[25,26]$.

The most suitable location of the spots for $\mathrm{U}-\mathrm{Pb}$ analyses was then selected. Zircon grains were also inspected after the isotopic and chemical analyses in order to define the precise spot location with respect to internal microstructures (Fig.3).

\subsection{In situ U-Pb data acquisition on zircon crystals}

$\mathrm{U}-\mathrm{Pb}$ age data on zircons were performed by LA-ICP-MS, SIMS and SHRIMP techniques. Three techniques produce comparable results with equally accurate $\mathrm{U}-\mathrm{Pb}$ zircon ages $[5,27,17]$. However, LA-ICP-MS technique is generally preferred for the greater simplicity of use, the faster data capture (ca 4 minutes per analysis versus ca 30 minutes per SIMS and SHRIMP analysis; see [5]) and because it allows a complete acquisition of trace element composition in selected zircon domains. 

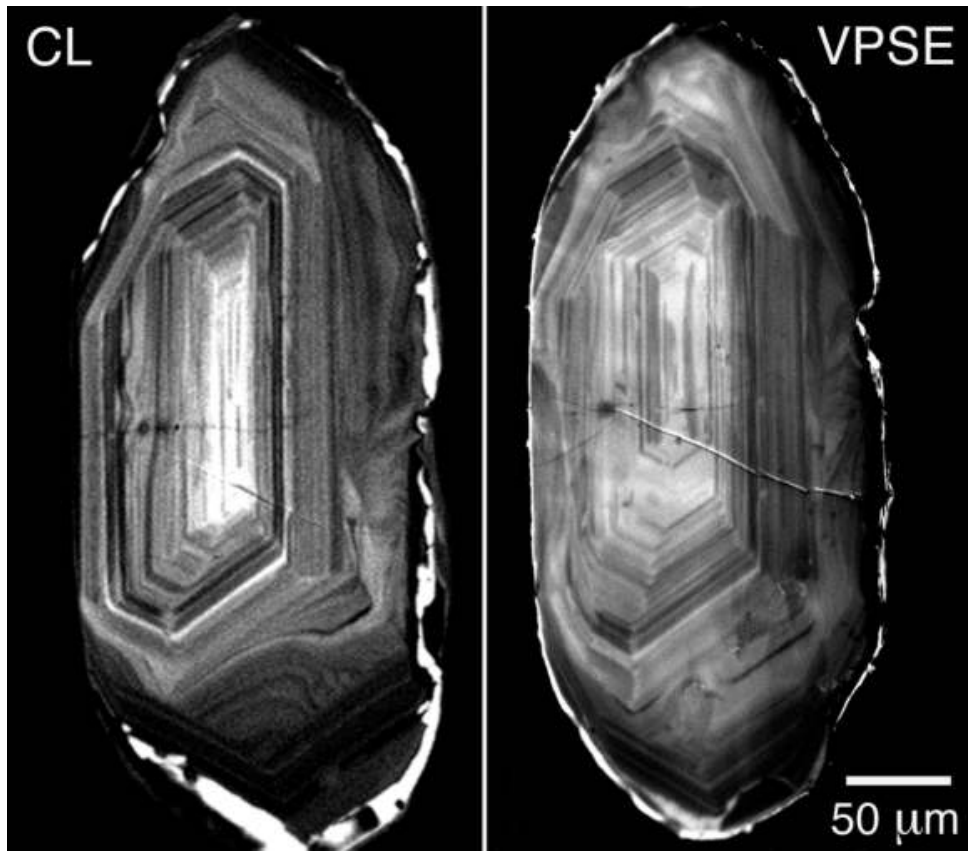

Figure 2. The same zircon crystal investigated by Cathodoluminescence Detector (CLD) and Variable Pressure Secondary Electrons Detector (VPSED). With the permission of Acquafredda and Fiore (2005).
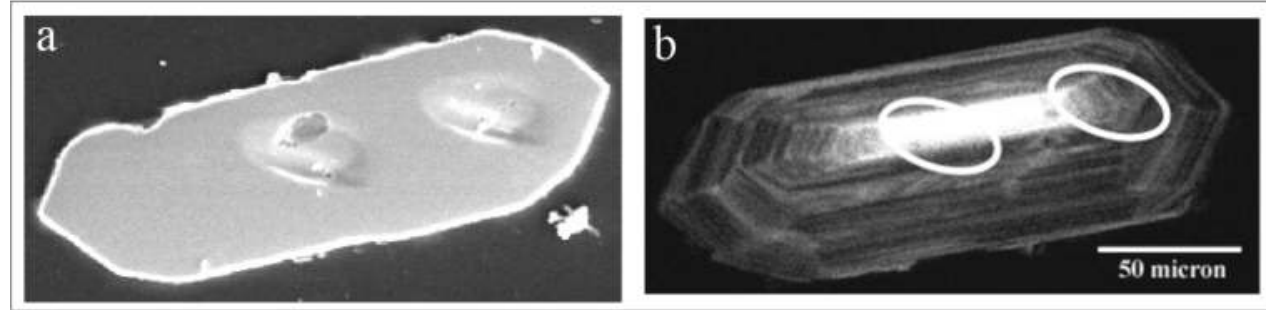

Figure 3. Images of the same zircon crystal obtained by BSE (a) and VPSE detectors (b). In (a) the craters left by microprobe are clearly visible.

\subsubsection{LA-ICP-MS (Laser Ablation-Inductively Coupled Plasma-Mass Spectrometry)}

LA-ICP-MS U-Pb data on zircons $(17,18,7,22,21,20,24)$ were performed at the CNR-Istituto di Geoscienze e Georisorse (Pavia, Italy) using a $193 \mathrm{~nm}$ ArF excimer laser ablation microprobe (Geo-Las200Q-Microlas) coupled to a magnetic sector high resolution-ICP-MS (Element I from Thermo Finnigan). 
The analyses were carried out in single spot mode and with a spot size of approximately $20 \mu \mathrm{m}$. The laser was operated with $5 \mathrm{~Hz}$ of frequency and 12J $\mathrm{cm}^{-2}$ of fluence. Sixty seconds of background signal and at least $30 \mathrm{~s}$ of ablation signal were acquired. The signals of masses ${ }^{202} \mathrm{Hg},{ }^{204}(\mathrm{~Pb}+\mathrm{Hg}),{ }^{206} \mathrm{~Pb},{ }^{207} \mathrm{~Pb},{ }^{208} \mathrm{~Pb},{ }^{232} \mathrm{Th}$ and ${ }^{238} \mathrm{U}$ were acquired in magnetic scan mode. ${ }^{235} \mathrm{U}$ is calculated from ${ }^{238} \mathrm{U}$ on the basis of the ratio ${ }^{238} \mathrm{U} /{ }^{235} \mathrm{U}=137.88$. The 202 and 204 masses were collected in order to monitor the presence of common $\mathrm{Pb}$ in zircon. In particular, the signal of ${ }^{202} \mathrm{Hg}$ was acquired to correct the isobaric interference of ${ }^{204} \mathrm{Hg}$ on ${ }^{204} \mathrm{~Pb}$ [28]. The relatively high $\mathrm{Hg}$ background, however, hampers the detection of low ${ }^{204} \mathrm{~Pb}$ signals and, as a consequence, also the calculation of small common $\mathrm{Pb}$ contribution. Generally, the analysed zircons showed signals of 204 mass elements, which were indistinguishable from the background; thus no common $\mathrm{Pb}$ correction was applied (more analytical details in [29]).

All fractionation effects involving $\mathrm{Pb} / \mathrm{U}$ ratios (e.g. mass bias and laser induced fractionation) were corrected by using the external zircon standard 91,500 (1,065 Ma [30]). The same spot size and integration intervals were considered on both standard and studied zircons. During each analytical run reference zircon 02123 (295 Ma [31]) was analysed together with unknowns for quality control. Data reduction was carried out through the GLITTER software package [32]. Time resolved signals were carefully inspected to detect perturbation of the signal related to cracks or mixed age domains that were avoided in the integration intervals.

Within the same analytical run, the uncertainty associated to the reproducibility of the external standards was propagated to each analysis (see Horstwood et al. 2003) and after this procedure each age determination is considered as accurate within a quoted uncertainty.

Correlation coefficients for the ${ }^{206} \mathrm{~Pb} /{ }^{238} \mathrm{U}$ and ${ }^{207} \mathrm{~Pb} / 235 \mathrm{U}$ data point uncertainties are calculated simply as a ratio of the two uncertainties [33].

LA-ICP-MS technique also permits a complete acquisition of trace element composition in specific zircon domains. In this case a dedicated configuration of the machine couples a Nd: YAG laser working at $266 \mathrm{~nm}$ with a quadrupole ICP mass spectrometer type DRCe from Perkin Elmer.

For trace element determination the laser was operated at a repetition rate of $10 \mathrm{~Hz}$, with pulse energy of about $0.01 \mathrm{~mJ}$ and an ablation spot of about $25 \mu \mathrm{m}$ in size. NBS NIST-610 and $\mathrm{SiO}_{2}$ were adopted as external and internal standard, respectively. Data reduction was carried out through the GLITTER software package [32].

Minimum detection limits at $99 \%$ confidence level were $<10-5$ ppm for the most of the elements. Precision and accuracy were assessed on the BCR-2 USGS reference glass and are more than $6 \%$ relative.

\subsubsection{SIMS (Secondary Ion Mass Spectrometry)}

SIMS data [16,17] were collected using the Cameca IMS-1270 ion microprobe (CRPGCNRS, Nancy, France). Primary O2- ion beam was accelerated at $13 \mathrm{kV}$ with an intensity that ranged between 5 and $20 \mathrm{nA}$ and focused on a 20-25 $\mu \mathrm{m}$ diameter area, providing 
a mean secondary ions yield of $25 \mathrm{cps} / \mathrm{nA} / \mathrm{ppm}$. Each spot was analysed for $18 \mathrm{~min}$, after a presputerring of $2 \mathrm{~min}$. An empirical relationship between $\mathrm{UO}+/ \mathrm{U}+$ and $\mathrm{Pb}+/ \mathrm{U}+$ was defined from all the measurements performed on the standard parts of each sample mount in order to determine the relative sensitivity factor for $\mathrm{Pb}$ and $\mathrm{U}$ used for samples [34]. Also in this case zircon 91,500 (1,065 Ma, [30]) is the reference standard. Correction for common lead was made measuring the $204 \mathrm{~Pb}$ amount. The common lead composition was calculated at ${ }^{207} \mathrm{~Pb} /{ }^{206} \mathrm{~Pb}$ measured ages, using Stacey and Kramers model [35]. The ${ }^{206} \mathrm{~Pb} /{ }^{204} \mathrm{~Pb}$ ratios range in most case from 3,000 to more than 50,000 , and thus the common $\mathrm{Pb}$ composition chosen for correction is not highly critical. Further information on instrumental conditions and data reduction procedures are found in [36]. Errors include the analytical statistical error, the error associated with the common lead correction and the systematic error associated with the $\mathrm{U} / \mathrm{Pb}$ calibration procedure [36]. As regards the ion microprobe analyses we used ${ }^{206} \mathrm{~Pb} / 238 \mathrm{U}$ ages for all the data instead of ${ }^{207} \mathrm{~Pb} /{ }^{235} \mathrm{U}$ ages, which are more sensitive to common lead contribution, being the ${ }^{207} \mathrm{~Pb}$ ion signal about ten times lower than the ${ }^{206} \mathrm{~Pb}$ ion signal [36].

\subsubsection{SHRIMP (Sensitive High Resolution Ion MicroProbe)}

SHRIMP data were collected [19,23] using procedures based on those described by [37]. A 2.5 $\mathrm{nA}, 10 \mathrm{kV}$ primary beam of O2-ions was focused to a probe of c. $25 \mu \mathrm{m}$ diameter. Positive secondary ions were extracted from the sample at $10 \mathrm{kV}$, and the atomic and molecular species of interest analysed at c. 5000 mass resolution using a single ETP electron multiplier and peak switching. The Pb isotopic composition was measured directly, without correction for the small mass dependent mass-fractionation (c. $0.25 \%$ per a.m.u.). Interelement fractionation was corrected using the TEMORA II reference zircon, using a $\mathrm{Pb} / \mathrm{U}-\mathrm{UO} / \mathrm{U}$ power law calibration equation [38]. The uncertainty in the $\mathrm{Pb} / \mathrm{U}$ calibration was $0,46 \% . \mathrm{Pb}, \mathrm{U}$ and Th concentrations were measured relative to SL13 reference zircon. Common Pb corrections were very small (most $<0.3 \mathrm{ppm}$ total $\mathrm{Pb}$ ), so all were made assuming that the common $\mathrm{Pb}$ was all laboratory contamination of Broken Hill galena $\mathrm{Pb}$ composition $\left({ }^{204} \mathrm{~Pb} /{ }^{206} \mathrm{~Pb}=0.0625,{ }^{207} \mathrm{~Pb} /{ }^{206} \mathrm{~Pb}=0.962\right.$, ${ }^{208} \mathrm{~Pb} /{ }^{206} \mathrm{~Pb}=2.23$; [39]). Corrections for the plots and isotopic data table were made using ${ }^{204} \mathrm{~Pb}$. Corrections for the calculation of mean ${ }^{206} \mathrm{~Pb} /{ }^{238} \mathrm{U}$ ages used ${ }^{207} \mathrm{~Pb}$, assuming the analyses to be concordant. Ages were calculated using the constants recommended by the IUGS Subcommission on Geochronology [40].

All collected data were traited by the software package Isoplot/Ex3.00 [41]. This software was used for the concordia test and the probability of concordance calculation, performed for each analytical spot from ${ }^{206} \mathrm{~Pb} / 238 \mathrm{U}$ and ${ }^{207} \mathrm{~Pb} / 235 \mathrm{U}$ ratios. The Isoplot/Ex3.00 software was also used to construct Concordia, possible discordia lines and probability density plots and to calculate the mean concordia ages for data clusters defined on the basis of: (i) statistical significance, (ii) the visual appearance on the Concordia plot, (iii) the peak distribution along the probability density plot, and (iv) the correspondence with specific internal microstructures of zircon. 


\section{Geological setting of case study}

The Calabria-Peloritani Terrane (CPT) represents an "exotic terrane" formed by a PreMesozoic basement consisting of different tectonic units affected by Variscan metamorphism and stacked during the Alpine orogenesis [42-43]. It carries the cristalline Massifs of Calabria (Sila, Serre and Aspromonte) and Peloritani Mountains in Sicily (Fig.4).

In the following, we describe the geological and petrological features of the four considered tectonic units representing portions of middle and lower continental crust. Many samples from the different rock types in each unit were considered with the aim to reconstruct and date the geological events recorded by zircon grains. The mineralogical composition of the all cited samples and their relative spectrum of $\mathrm{U}-\mathrm{Pb}$ zircon ages are reported in Table 1 . Their exact localization is indicated in Fig.4.

Four tectonic units were considered along the CPT from North to South (Fig. 4):

The Mandatoriccio Complex outcropping in Sila Massif represents intermediate continental crust and consists mainly of metapelites, meta-arenites, acidic metavolcanites and metabasites with rare intercalations of marbles and orthogneisses crosscutted by abundant aplite and pegmatite veins [44-46]. Metasediments show a static porphyroblastic growth mainly of biotite, garnet, andalusite, staurolite and muscovite [46-48]. Recently, clockwise P-T paths have been constrained for siliciclastic metasediments of this complex. Peak-metamorphic conditions of ca. $590{ }^{\circ} \mathrm{C}$ and $0.35 \mathrm{GPa}$ are reported for the lower structural levels of the Mandatoriccio Complex and they were reached at $299 \mathrm{Ma}$ (U-Th-Pb ages in monazite, [46]) during Variscan post-orogenic extension.

The Castagna Unit outcropping in the central part of Calabria, consists of paragneisses, micaschists, augen gneisses, Variscan granitoids and minor amphibolites, quartzites, calcsilicate rocks and marbles [49,50]. It includes metamorphic rocks equilibrated under greenschist to amphibolite facies conditions in Variscan times and reworked by Alpine tectonics $[49,46,18]$.

The Sila Unit occupies wide areas in Sila and Serre Massifs. In the Serre the section of Variscan crust consists, from the bottom to the top of: i) 7-8 km thick lower crustal rocks, ii) an about $10 \mathrm{~km}$-thick "layer" of granitoids [51] emplaced $300 \mathrm{Ma}$ ago (U-Pb conventional zircon data, [51,52]), and iii) amphibolite to sub-greenschist facies metamorphic rocks of the upper crust. The geochronological features of the lower portion recording Variscan amphibolite-granulite facies metamorphism and representing a fragment of deep crust were considered. This section includes from the bottom: a) felsic and mafic granulites with rare meta-peridotites and metapelites, b) migmatitic metapelites with interleaved metabasites, rare marbles and augen gneisses. The metabasic rocks from the lower part of the section together with the augen gneisses from the upper part of the section bear memory of preVariscan magmatism (Table 1).

The Aspromonte-Peloritani Unit (APU) outcrops in Southern Calabria and Eastern Sicily (Fig. 4); it consists of augen gneisses, micaschists, biotite paragneisses with minor amphib- 
olites and marbles. A pre-Variscan origin of protoliths of augen gneisses at 543-545 Ma was suggested by spot U-Pb zircon ages [16,23,53]. According to [23] the timing of highgrade metamorphism accompanied by partial melting in paragneisses, was sinchronous (at $545 \mathrm{Ma}$ ) with the intrusion of protoliths of augen gneisses. The metamorphic rocks are diffusely intruded by late-Variscan peraluminous granitoids [54-57,19], sometimes affected by Alpine metamorphism. P-T estimates for the Variscan tectono-metamorphic evolution indicate $\mathrm{T}$ around $650-675^{\circ} \mathrm{C}$ and $\mathrm{P}$ of about $0.4-0.5 \mathrm{GPa}[58,59]$. The evolution of the APU provides crustal thickening during early-middle Variscan collisional stages, followed by crustal thinning, granitoid intrusion and unroofing during late-Variscan extensional stages [43].

The collected data in Southern Italy give constraints about the complex magmatic and metamorphic history of South European Variscan Chain in the peri-Mediterranean area. Magmatic and metamorphic events are recorded in the considered rocks from Late-Neoproterozoic-early Cambrian to Permian times. In the following, the geological history is punctually depicted.

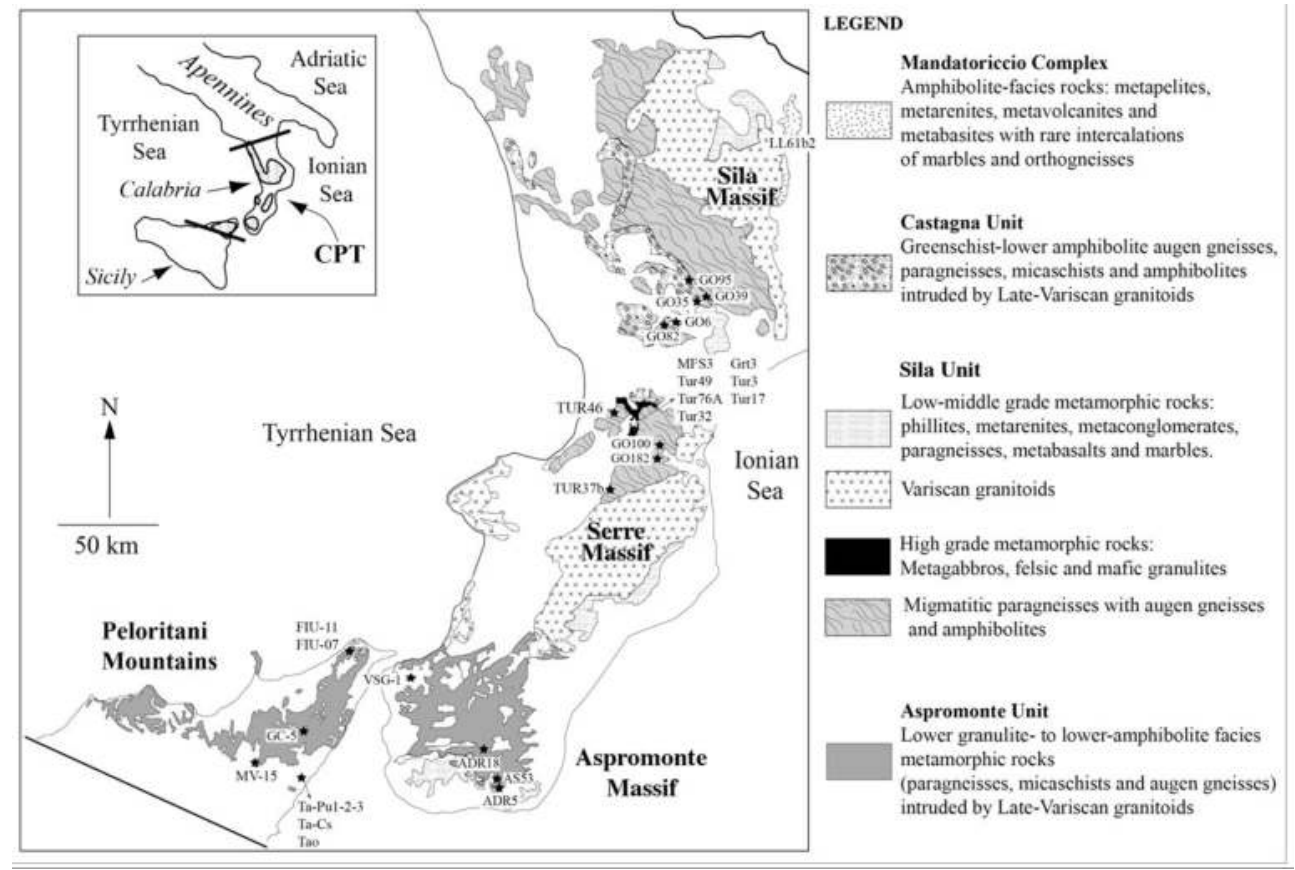

Figure 4. - Geological sketch map of CPT with the indication of considered samples in four different structural units. 


\begin{tabular}{|c|c|c|c|c|c|c|c|c|c|c|c|}
\hline 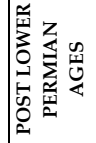 & & 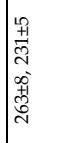 & 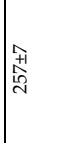 & & 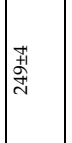 & & 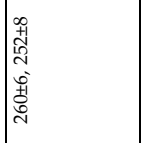 & & & & \\
\hline 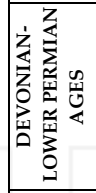 & & 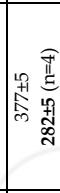 & 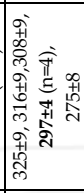 & 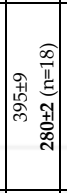 & 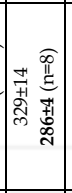 & 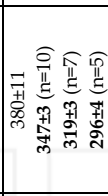 & 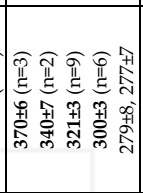 & 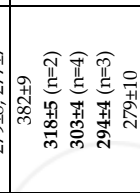 & 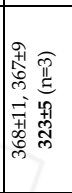 & 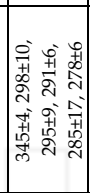 & 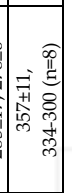 \\
\hline 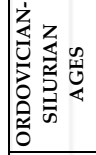 & 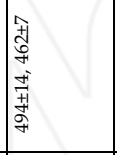 & 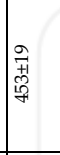 & $\begin{array}{l}\text { के } \\
\text { ô } \\
\text { के }\end{array}$ & & & 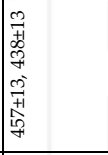 & 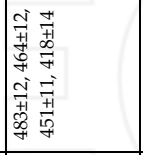 & & & 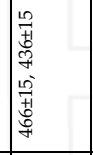 & \\
\hline 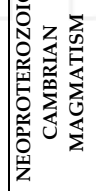 & 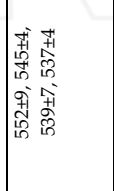 & 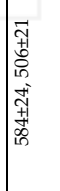 & & & & $\begin{array}{l}\infty \\
\text { 京 } \\
\text { 离 }\end{array}$ & 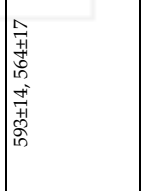 & 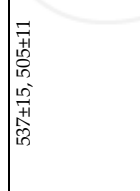 & & & \\
\hline 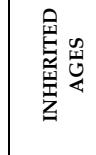 & 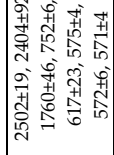 & & 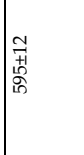 & & & 勇 & & 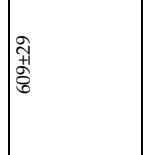 & & & \\
\hline 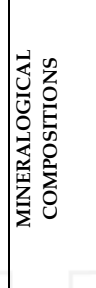 & 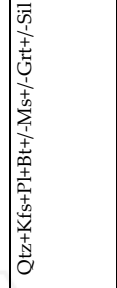 & 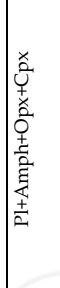 & 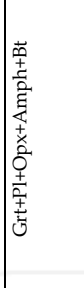 & 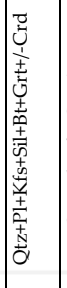 & 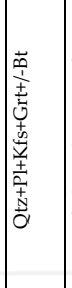 & 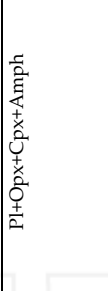 & 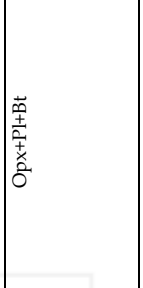 & 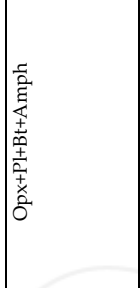 & 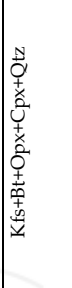 & 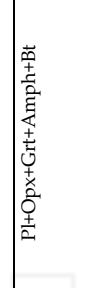 & 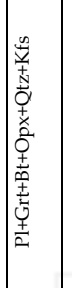 \\
\hline 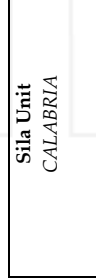 & 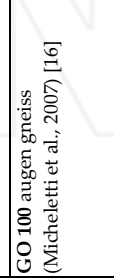 & 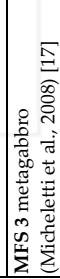 & 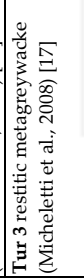 & 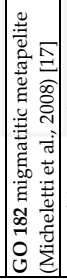 & 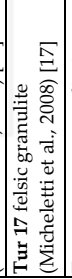 & 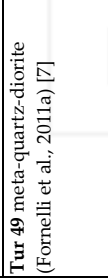 & 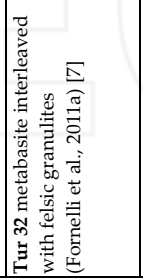 & 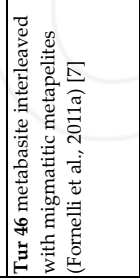 & 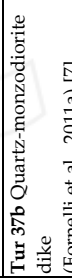 & 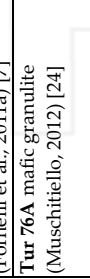 & 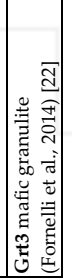 \\
\hline
\end{tabular}




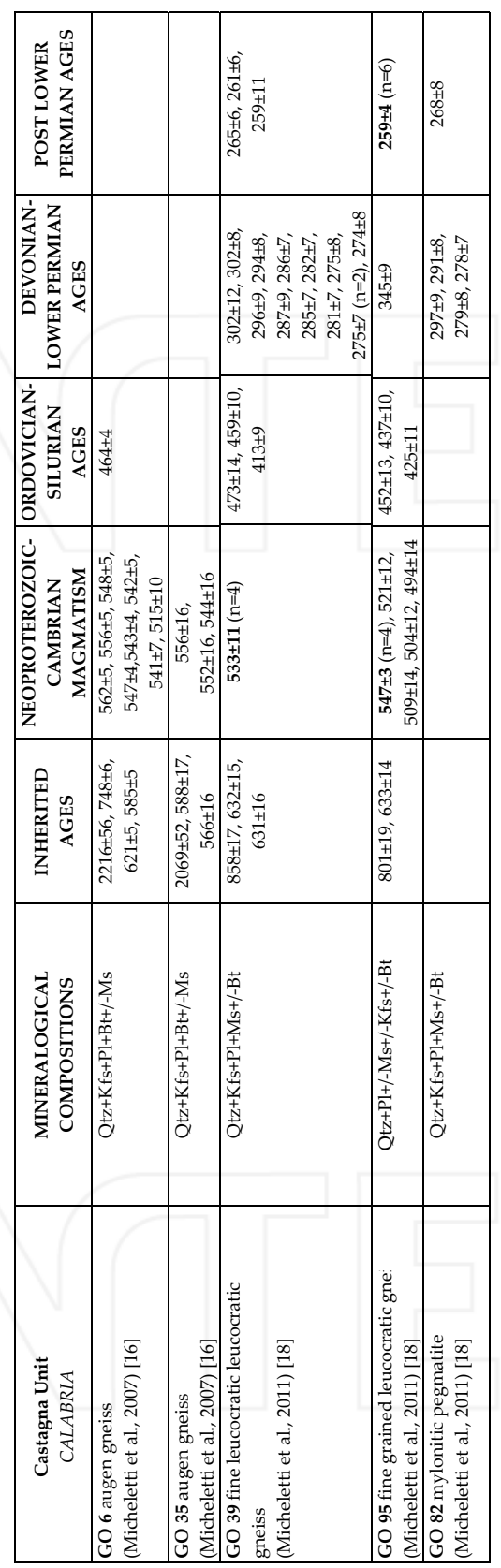

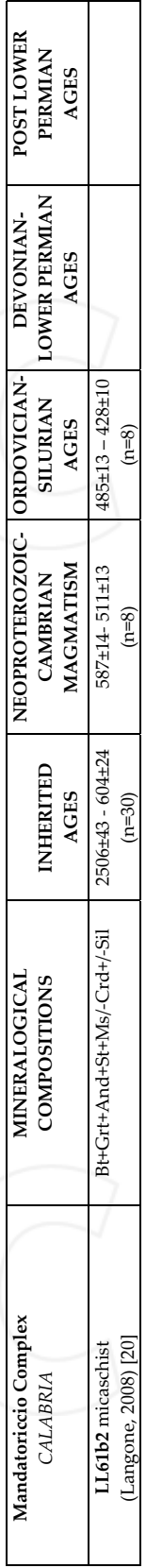



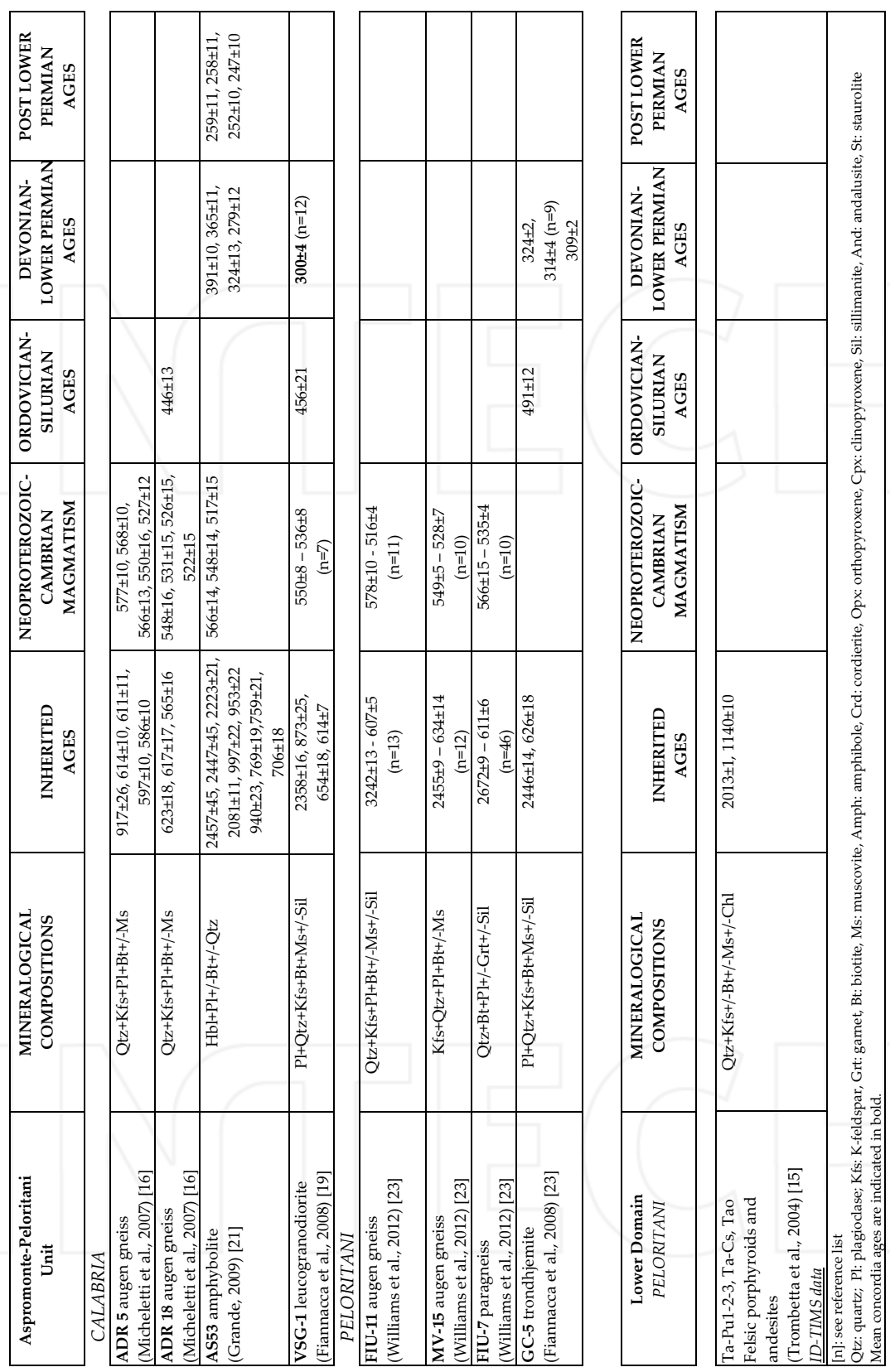

Table 1. U-Pb concordant data on zircon in the studied rocks from CPT continental crust 


\subsection{Geological reconstructions inferred from $\mathrm{U}-\mathrm{Pb}$ spot data}

\subsubsection{Recognizable Gondwana pertinence}

Memory of the magmatic Proterozoic events widely occurs in the rocks of the CalabriaPeloritani Terrane as inherited ages preserved in detrital zircons or xenocrystic cores surrounded by younger overgrowths (Fig. 5).

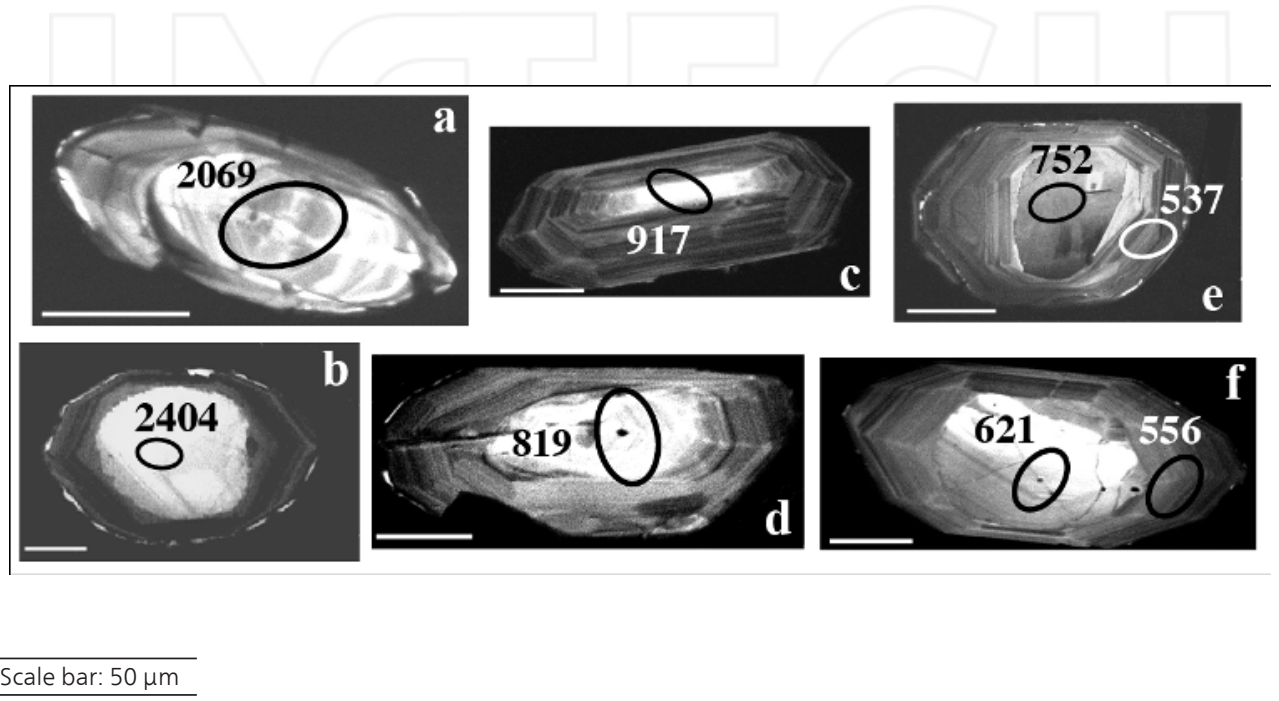

Figure 5. CL images of zircons showing detritic characters (a-b-c-d) and xenocrystic cores (e-f). Note the younger overgrowths in e and $f$.

The zircon ages revealed in the different rock-types of the described units are detailed in the following:

- Metagranitoids (augen gneisses) belonging to Aspromonte-Peloritani, Castagna and Sila Units having Fortunian-Ediacaran protoliths (Table 1) preserve inherited zircon ages covering a time span of 2500-1760 Ma and 917-610 Ma [16,18]. In addition, they show Sm$\mathrm{Nd}$ model ages ranging from 1700 to $1500 \mathrm{Ma}$ [16];

- Granulite facies metabasic rocks of the Sila Unit record two Neoproterozoic inherited age (744 $\pm 20 \mathrm{Ma}$ and $609 \pm 29 \mathrm{Ma}$ ) despite their magmatic mantle origin in the NeoproterozoicCambrian times [7];

- Metasediments from the lower and upper crust of the Sila Unit preserve memory of old events at 2500, 2300, 2000 and 1900 Ma obtained as upper intercepts of U-Pb discordia lines (isotopic dilution method in [60,61]). Furthermore they show Nd model ages of $1500 \mathrm{Ma}$ [60] and $1350 \mathrm{Ma}$ [61]; 
- Amphibole bearing-micaschists from Mandatoriccio Unit preserve xenocrystic cores old up to $2562 \pm 44$ Ma [20];

- Amphibole bearing-gneisses from Aspromonte-Peloritani Unit preserve inherited ages from 2500 Ma to $706 \mathrm{Ma}$ [21];

- In Eastern Sicily ortho-and para-gneisses belonging to APU show clusters of Proterozoic ages around 2400-2700 Ma, 900-1100 Ma and 540-850 Ma and some ages at 3200, 1800 and 1600 Ma were also obtained [23].

Still in Eastern Sicily (Lower domain) Ordovician porphyroids preserve inherited ages at $1512 \mathrm{Ma}$ and $1380 \mathrm{Ma}$ (upper intercepts of U-Pb discordia lines; ID-TIMS analyses in [15]). Meta-andesites associated with these porphyroids contain older zircons dated at 2015 and $1150 \mathrm{Ma}[15]$.

The inherited zircon age patterns in Calabria rocks indicate Gondwana domain pertinence. In particular, the similarities in age and chemistry of the protoliths of Calabria augen gneisses with the acidic magmatites from Anti-Atlas Moroccan domain point to a source derived from a reworking of the West African Craton (WAC) [48] showing similarities with other European Cadomian terranes [62-66]. In fact, analogous inherited age patterns occur in other sectors of European Variscides [63]: Southern British Islands, Armorica Massif and Massif Central in France, Bradant Massif (Belgium), Iberian Massif (Spain and Portugal) and Bohemian Massifs (Czech Republic). The distribution of these ages suggests Cadomian pertinence with West African Craton affinity. According to [23], the inherited zircon age patterns in the augen gneisses and paragneisses from Aspromonte-Peloritani Unit in the Sicily bear evidence, on the whole, of East African provenance owing to age cluster in the range 900-1100 Ma reveled in this area. These authors evidence strong similarities between Sicily terranes and those of other areas in the Eastern Mediterranean region as Southern Israel, Jordan and Arabia and suggest an East African provenance as several pieces of basements in Mediterranean area, like Turkey, Greece, Sardinia and Cyclades [23]. Considering the whole set of inherited zircon ages in Calabria and Sicily, we can suggest that a both East and West African provenance was effective in Southern Italy as happened in other Mediterranean areas where pre-Variscan basements, starting with Neoproterozoic, are considered as derived from both East and West Gondwanan cratonic sources $[67,68]$. The presence of detritic components having West and East African origin was reveled by inherited zircon ages acquired with different techniques in many samples from different domains of Calabria and Sicily terrains, revealing the effectiveness of $\mathrm{U}-\mathrm{Pb}$ zircon data.

\subsubsection{Neoproterozoic-Cambrian bimodal magmatism}

In situ U-Pb dating performed in metaigneous rocks on zircon crystals showing euhedral morfology and a typical undisturbed oscillatory zoning [69] (Fig. 6) has evidenced a widespread Neoproterozoic-Cambrian bimodal magmatism in an older basement of CalabriaPeloritani Terrane. Evidences of basic and acidic magmatism are diffused in Calabria from Sila Massif up to Sicily (Fig. 4) in metabasic rocks, augen gneisses, fine-grained leucocratic gneisses and amphibolites (Table 1, [16-18,7,23,21]). 

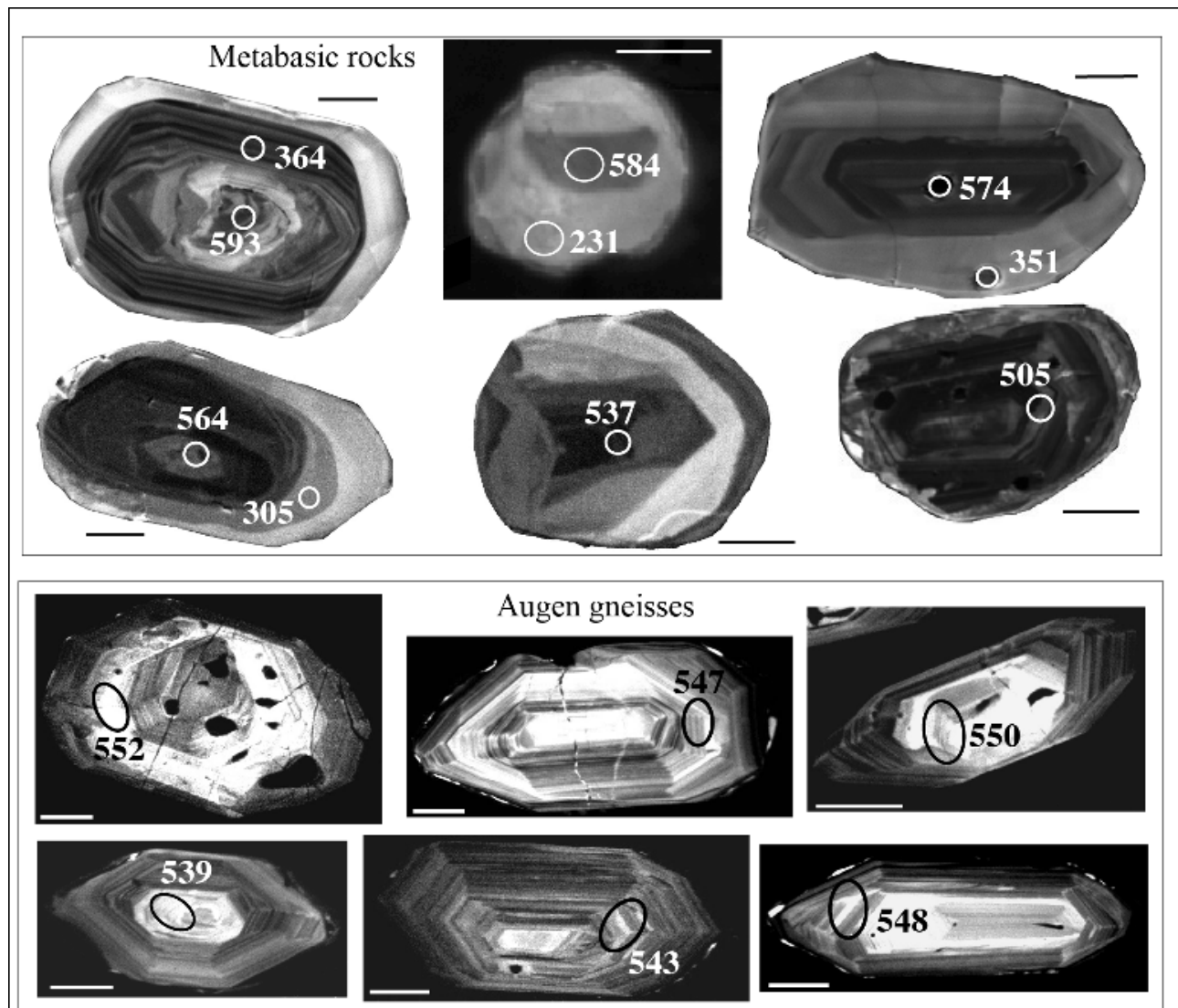

Fine grained leucocratic gneisses
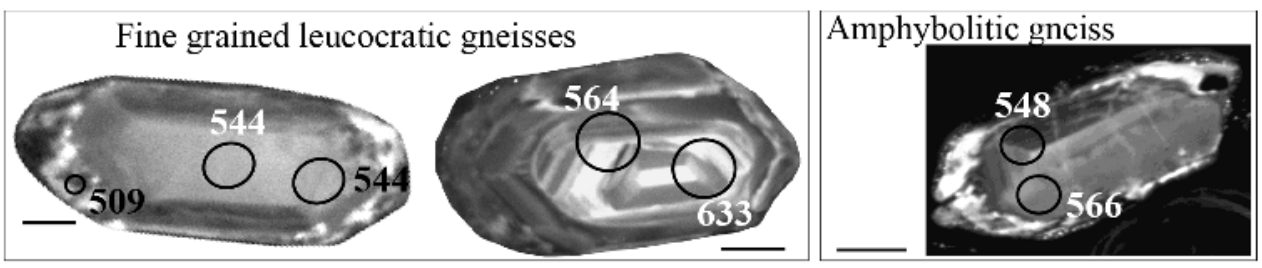

Figure 6. Cathodoluminescence images of zircons in metabasic rocks, augen gneisses, fine grained leucocratic gneisses and amphybolitic gneisses showing characteristic oscillatory zoning interpreted as indicative of their magmatic origin. Scale bar: $50 \mu \mathrm{m}$

Zircons from 2 samples of metagabbro and from 2 samples of metabasites interbedded with felsic granulites and migmatitic metapelites of the lower crust of the Serre Massif belonging to Sila Unit were dated [17,7]. In all samples domains dated 564-593 Ma $(n=4)$ showing magmatic oscillatory zoning and high Th/U ratio (0.16-0.19) are present (Fig. 6). These domains 
show fractionated REE patterns interpreted as formed in absence of garnet considered as Variscan metamorphic phase $[7,70]$. On this basis a magmatic origin of the zircons indicating the age of protoliths in the time range 564-593 Ma was suggested [17,7]. So, a basic magmatism in Calabria occurred in Neoproterozoic times in an older basement, as happened in many of the so-called "Cadomian blocks" widespread from Western Alps to Turkey [71].

This basic magmatism records tholeiitic and calc-alkaline affinities and, due to the association with a thick pile of metasediments, seems to be connected with a (mature?) magmatic arc in orogenic context [72].

Zircon grains from seven samples of biotitic augen gneisses and two samples of fine-grained leucocratic gneisses coming from the Aspromonte-Peloritani (4 samples), Sila (1 sample) and Castagna (4 samples) Units in Calabria [16,18] and Eastern Sicily [23] are considered. These gneisses are intimately associated with metasediments affected by Variscan metamorphism, but their zircon domains do not bear memory of this event, preserving only Pre-Cambrian/ Silurian ages [16,18,23]. Only one sample (GO39, Table 1) preserves Devonian-Lower Permian ages interpreted as resetted ages due to thermal input of fluids relased by Late-Variscan plutonites [18].

In the Calabria augen and fine grained leucocratic gneisses, the majority of the concordant ages forms a statistically significant cluster averaging at 543 Ma ( $n=20$ ages from 562 to 532 Ma) mainly related to euhedral crystals without discontinuity between core and rim having $\mathrm{U}$ contents ranging from 659 to $241 \mathrm{ppm}$ and Th/U ratios mostly comprised between 0.2 and 0.5 ; one domain analysed for REEs produces a highly fractionated pattern and a distinct negative Eu anomaly [18] interpreted as primary magmatic characters according to [73] or as recrystallized domains with memory of primary magmatic zircons [74]. The moderate variability and the high values of $\mathrm{Th} / \mathrm{U}$ ratios seem to be more compatible with precipitation from a hybrid magma precursor of the augen gneisses [75] having mantle and crustal origin. Discordia lines with lower intercepts comprised between 562 Ma and 526 Ma have been also calculated considering the discordant data [16].

The augen gneisses from Peloritani Mountains contain zircon grains giving ages around 545 Ma including two kinds of zircon domains having $\mathrm{U}$ contents of 320-940 ppm (Th/U=0.08-0.23) and 40-470 ppm $(\mathrm{Th} / \mathrm{U}=0.12-2.32)$ interpreted as suggestive of magmatic and detritic origin, respectively [23]. On this basis [23] suggest that the protoliths of augen gneisses were the hosting metasediments in which similar ages were detected.

This acidic magmatic activity dated around 543-545 Ma seems to be diffused in the CalabriaPeloritani basement successively than basic magmatism described above.

Chemistry of the augen gneiss indicates that their protoliths derived from shoshonitic to highK calc-alkaline granitoids related to a post-collisional stage [16, 76], probably at the transition from compressional to extensional tectonics or even after the tectonic collapse of an intracontinental orogen [75]. The emplacement age obtained from the protoliths of all granitic gneisses in CPT and their geochemical affinity share similarities with the granitoids widespread at the Northern edge of the West African Craton, especially in Morocco [77-79], Algerian Tuareg Shield [80] and Mauritania [81]. In fact, voluminous high-K calc-alkaline plutonism charac- 

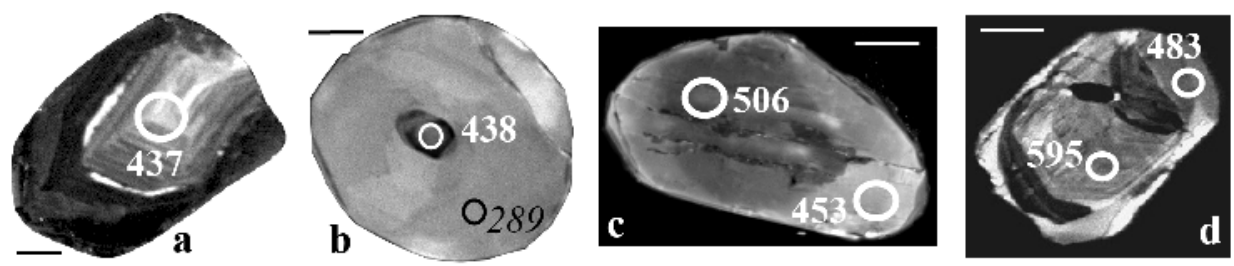

Figure 7. $C L$ and VPSE images of selected zircons showing Ordovician-Silurian ages in fine grained leucocratic gneisses (a), metagabbros (b, c) and restitic metagreywake (d). Scale bar: $50 \mu \mathrm{m}$.

terize the final stages of Panafrican orogeny in Northern margin of the West African Craton as well as in almost all the Cadomian Units along the present Alpine-Mediterranean mountain belts [81,82]. In the period 605-530 Ma, acidic magmatism was diffused at the transition from an active (compressive-transtensive) to a passive (extensional) continental margin with extension and development of foreland basins [83].

It is noteworthy that acidic and basic magmatism of Neoproterozoic-Lower Cambrian times, in Calabria-Peloritani Terrane, is diachronous being mafic magmatic activity 20-40 Ma older than the acidic one [16-18,7]; both magmatic activity monitored the tectonic evolution of Panafrican orogen from compressional to collapse stages [72].

\subsubsection{Ordovician-Silurian tectono-thermal activity}

Ordovician-Silurian ages (data ranging from $494 \pm 14$ to $413 \pm 9 \mathrm{Ma}$ ) have been recorded in augen gneisses, fine-grained leucocratic gneisses and granulite-facies metabasites from Calabria (Table 1) [16-18, 7, 24]. In these rock-types the Ordovician-Silurian ages represent clusters connected to a recrystallization event being the protoliths Neoproterozoic-Cambrian in origin. These ages were measured on cores displaying irregular and patchy microstructures sometimes strongly luminescent (Fig. 7 a-b) or on overgrowths surrounding older cores (Fig. 7 c-d). Owing to the textural features of zircons, the Ordovician-Silurian ages seem related to a tectonothermal event as an effect of recrystallization (see $[84,85])$ producing an isotope resetting at that time.

One sample of augen gneiss (sample GO100, Table 1) from Sila Unit interleaved with the migmatitic metapelites shows two Ordovician ages at $494 \pm 14 \mathrm{Ma}$ and $462 \pm 7 \mathrm{Ma}$ as a $\mathrm{Rb}-\mathrm{Sr}$ isochron at $450 \pm 20 \mathrm{Ma}$ determined in migmatitic metapelites [86]. In addition OrdovicianSilurian detritic population of zircon occurs in the Mandatoriccio micaschists in Mandatoriccio Complex (sample LL61b2, Table 1). In Peloritani Mountains an intermediate-acidic magmatism in Ordovician times was revealed by [15] analysing zircons with magmatic textures from porphyroids and meta-andesites dated at ca. 456-452 Ma.

A look at the European Variscan Chains in which Ordovician-Silurian ages have been detected reveals that from Iberian Massifs to Carpathians several acidic and mafic products are related to a diffusely Ordovician magmatic activity [68]. According to [67,68,71] rifting phases in the Early and Middle Palaeozoic prepared the opening of basins separating the future Variscan 
basement from Gondwana. If a tectono-metamorphic phase was responsible of magmatic activity in Ordovician-Silurian times recorded in Variscan fragments of European Chain, then also the $\mathrm{U}-\mathrm{Pb}$ zircon ages determined in Calabria-Peloritani rocks can be referred to the same phase. Nevertheless, it cannot be excluded that these ages might result from rejuvenation due to the opening of $\mathrm{U}-\mathrm{Pb}$ radiogenic system with partial loss of $\mathrm{Pb}$ during the Variscan metamorphism [16,18]. Alternatively, according to the model proposed by [87], these ages can be related to an Eo-Variscan activity started in Silurian-Ordovician times.

\subsubsection{Variscan orogenesis (Devonian-Lower Permian times)}

The investigated basement forming continental crust units of Calabria and Sicily was affected by Variscan metamorphism and magmatism [88,42] as shown by $\mathrm{Rb}-\mathrm{Sr}$ and $\mathrm{Sm}-\mathrm{Nd}$ isotopic geocronology $[89,61]$. U-Pb zircon age data can be utilized to evidence these geological processes realized under high temperature conditions; in fact zircon has very high closure temperature for $\mathrm{U}-\mathrm{Th}-\mathrm{Pb}$ isotopic system $\left(>900^{\circ} \mathrm{C}\right.$ in [90-92]) then only high-T metamorphic and magmatic conditions can be monitored through $\mathrm{U}-\mathrm{Pb}$ zircon data. Zircons of amphibolitic facies paragneisses and micaschists from Mandatoriccio Complex in Calabria [20] and from Aspromonte-Peloritani Unit in Sicily [23], respectively, do not evidence Variscan ages owing to their low temperature metamorphic conditions (around $500-650^{\circ} \mathrm{C}$; $[20,93]$ ). Zircon is unefficient in these rock types to record geological events under low temperature conditions. This fact is confirmed in the augen gneisses from Castagna and Aspromonte-Peloritani Units (low-medium grade Variscan metamorphism) where ages younger than $413 \mathrm{Ma}$ in Calabria [16] and $\sim 516 \mathrm{Ma}$ in the Sicily [23] were not detected.

Mafic and felsic granulites together with migmatitic metapelites from Sila Unit in Calabria show many U-Pb zircon ages ranging from $\sim 380 \mathrm{Ma}$ to $280 \mathrm{Ma}[17,7]$ testifing the strong efficiency of high grade metamorphism in Variscan times that, in part, masks the original Neoproterozoic-Cambrian ages.

Zircons from these rock types record domains with oscillatory zoning generated by dissolution/re-precipitation or crystallization in presence of melts $[85,94]$ together with lobate structurless grey or luminescent rims invading older cores (metamorphic re-crystallization in [6]) (Fig. 8). The evaluation of zircon textural features on which spot ages were determined constrains step by step the Variscan metamorphic trajectory of the lower crust of the Sila Unit.

Distinct U-Pb zircon age clusters were determined (Table 1): i) a few ages from 380 Ma to 347 Ma; ii) 13 data points around 347-340 Ma; iii) 23 zircon ages clustering at $320 \mathrm{Ma}$; iv) 31 ages around $300 \mathrm{Ma}$; v) several ages in the range 270-280 Ma.

Considering the P-T evolution of the lower crust of the Sila Unit representing a fragment of Variscan continental crust, the revealed cluster ages were interpreted in the following [95]:

a. The ages from $380 \mathrm{Ma}$ to $347 \mathrm{Ma}$ indicate phases of crustal thickening during the prograde metamorphism from amphibolite to granulite facies;

b. The cluster at $347 \mathrm{Ma}$ represents the metamorphic peak at $\mathrm{T}=880^{\circ} \mathrm{C}$ and $\mathrm{P}=1.1 \mathrm{GPa}$ under granulite facies conditions in the lower part of the continental crust section; 
c. The age peaks at 320, 300 and $280 \mathrm{Ma}$ (Table 1; Fig. 8) date the decompression phases. In particular, the cluster at $320 \mathrm{Ma}$ in the granulites coincide with the age of basic Variscan magmatism determined on zircons of a quartz-monzodioritic dike dated at $323 \mathrm{Ma}$ (sample Tur37b, Table 1, Fig.9a). The first decompression stage was accompanied by partial melting in the hosted rocks as shown by zircon domains with oscillatory zoning crystallized by partial melt (Fig. 8). The age peak at about 300 Ma dates a further decompression phase and probably the end of anatexis in the granulites as testified by successive homogeneous and luminescente rims of zircons with ages around $280 \mathrm{Ma}$ in which the Variscan cycle stoped [7].

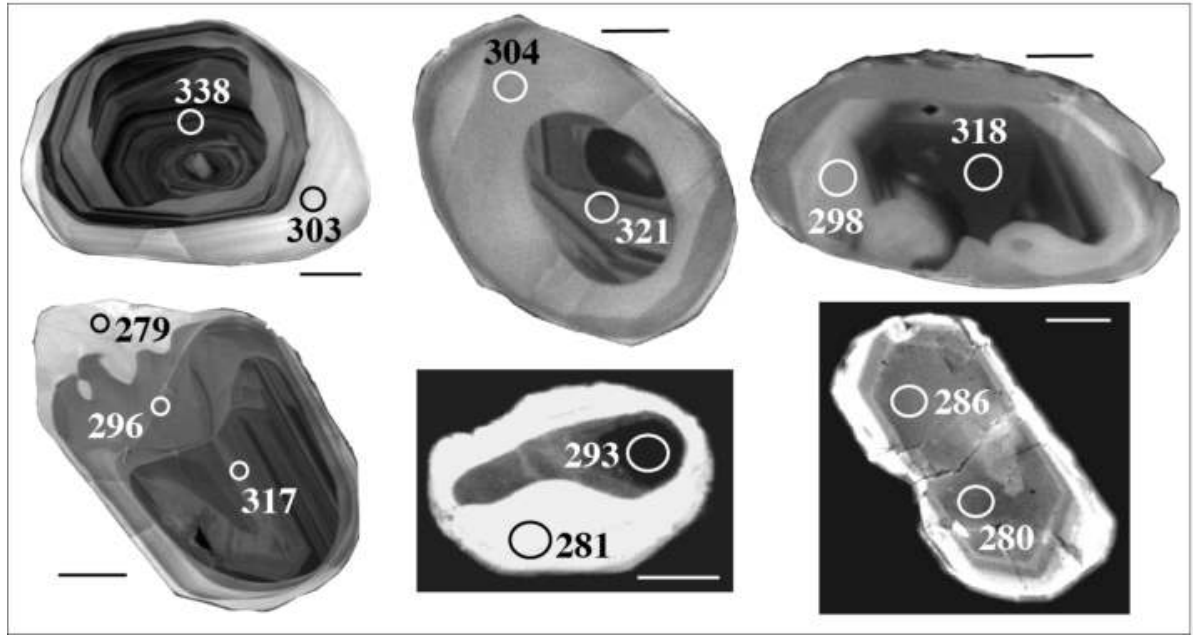

Figure 8. VPSED images of selected Variscan zircons. See the oscillatory zoning domains surrounded or invaded by lobate structurless grey or luminescent rims. Scale bar: $50 \mu \mathrm{m}$.

During the crustal thinning and decompression, emplacement of huge Late-Variscan calcalkaline granitoids occurred:1) between the upper and lower crustal portions of the Sila Unit (Fig. 4) at about $300 \mathrm{Ma}$ ago as showed by $\mathrm{Rb}-\mathrm{Sr}$ isotopic ages [52] and U-Pb zircon ages in granodorities and tonalities of the Serre batholite [96], 2) in the Castagna Unit as showed by $\mathrm{U}-\mathrm{Pb}$ dating of zircons in a pegmatitic dike (sample GO82, Table 1, Fig. 9b) and 3) in CPU as showed by $\mathrm{U}-\mathrm{Pb}$ zircon ages from peraluminous magmatites [19] (Table 1).

The geological evolution of the continental crust in Calabria was detailed utilizing the precious textures of zircons and the $\mathrm{U}-\mathrm{Pb}$ zircon data; the reconstructed scenario is confirmed by the comparison with similar metamorphic evolution of other lower crust fragments from the South European Variscides cropping out in the West Mediterranean areas [97-101]. 


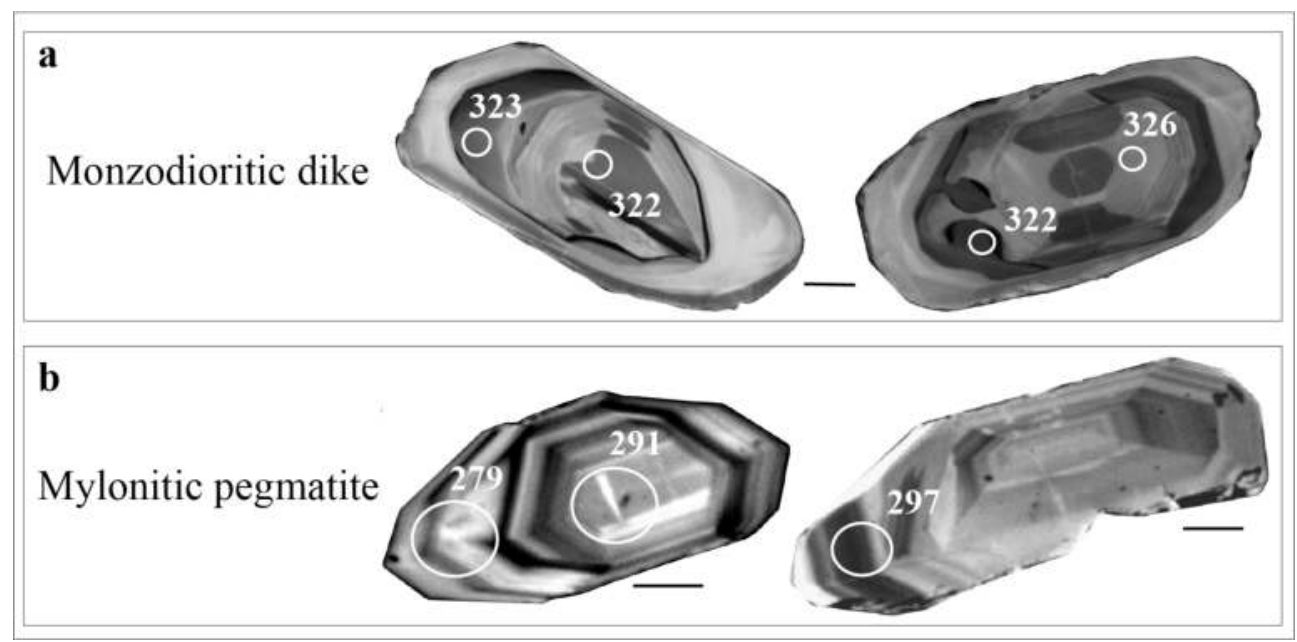

Figure 9. Magmatic zoning (VPSE images) in Variscan magmatites. Scale bar: $50 \mu \mathrm{m}$.

A limitation of the $\mathrm{U}-\mathrm{Pb}$ spot analyses on zircon is the spot size; in fact the augen gneisses interleaved with the migmatitic metasediments of the lower crust of the Serre (Calabria) show a thin recrystallized rim clearly shown by the cathodoluminescent images but undatable for the small size. Speculatively, these thin rims have been interpreted as formed during the Variscan metamorphism by [16], but their precise ages are not known.

\subsubsection{Post lower Permian events}

Few and scattered zircon ages comprised between 268 \pm 8 Ma and 231 \pm 5 Ma [72] (Table 1, Fig. 10) were measured in the granulites of the Sila Unit and in metamorphites and magmatites of the Castagna Unit. These ages have been interpreted as effect of a recrystallization event assisted by fluids [16,18,7]. A comparison with Variscan basements from Corsica [100] and Western Alps as the Ivrea zone [102,103] show similar cluster ages interpreted as precursor signals of the opening of the Tethys Ocean. An analogous interpretation can be adopted for the Calabria rock types associated to domains formed during the opening of Tethys Ocean. However, it can not be excluded that these ages in Calabria might be connected to opening of $\mathrm{U}-\mathrm{Pb}$ isotopic system of zircon due to Alpine tectonism [18], in fact the studied rocks belong to tectonic units stacked during the construction of Alpine chain and are affected by Alpine shear zones $[49,46]$. 


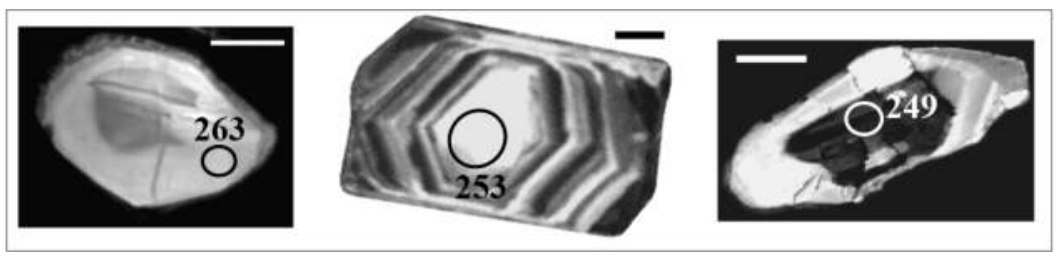

Scale bar: $50 \mu \mathrm{m}$

Figure 10. VPSED images of selected zircons showing post Lower-Permian ages.

\section{Conclusions}

The reconstruction of the pressure-temperature-time (P-T-t) evolution of crustal sections is fundamental to understanding many tectonic processes. This task, particulary difficult in the case of polymetamorphic rocks, requires the combination of metamorphic petrology and geochronology of different mineral phases that potentially can record more than one geological event. Zircon has been largely used for this role in high-grade terrains because its U-Pb system is able to retain the memory of polyphase evolution even at relatively high temperatures for its highly refractory nature, high closure temperature and slow $\mathrm{Pb}$ diffusion rate. Zircon is an ideal mineral for U-Pb dating of poly-metamorphic rocks [90,104-106,8,107-113]. In addition, the precise and accurate dating of the retrograde metamorphism is crucial for understanding the exhumation history of the ancient metamorphic basements. Obviously, spot U-Pb zircon data in magmatites formed under high temperature conditions, constrains the timing of magma emplacement and bring light on the geological context in which the magmatism explicated.

The case study presented in this paper shows as in situ zircon dating linked with determined $\mathrm{P}-\mathrm{T}$ conditions could constrain the evolution of the Calabria-Peloritani Terrane, a crucial fragment of Southern European Variscan Belt.

In the last ten years the advances in analytical capabilities have permitted in-situ investigation of complex zircon grains that allow us to reconstruct the geological history from Neoproterozoic-Cambrian to post Permian times in Southern Italy. In Fig.11 a histogram and a probability density curve of the U-Pb spot zircon ages collected in CPT are reported showing the large number of determinations in a wide time interval from Archean to Triassic ages.

The collected data are interpreted as suggestive of: (1) Neoproterozoic detrital input from cratonic areas of Gondwana testified by inherited zircons; (2) diachronic bimodal basic and acidic magmatism between 570 and $526 \mathrm{Ma}$, relative to an active tectonic margin setting; (3) rifting and opening of Ordovician-Silurian basins signed by consistent cluster ages around 450 Ma corresponding to acidic and intermediate volcanic activity (porphyroids and metaandesites in Peloritani Mountains); 4) Variscan granulite facies metamorphism and pervasive partial melting in deep crustal rocks of the Sila Unit; 5) precursor signals of the Tethys evolution showed by post Permian zircon domains. 


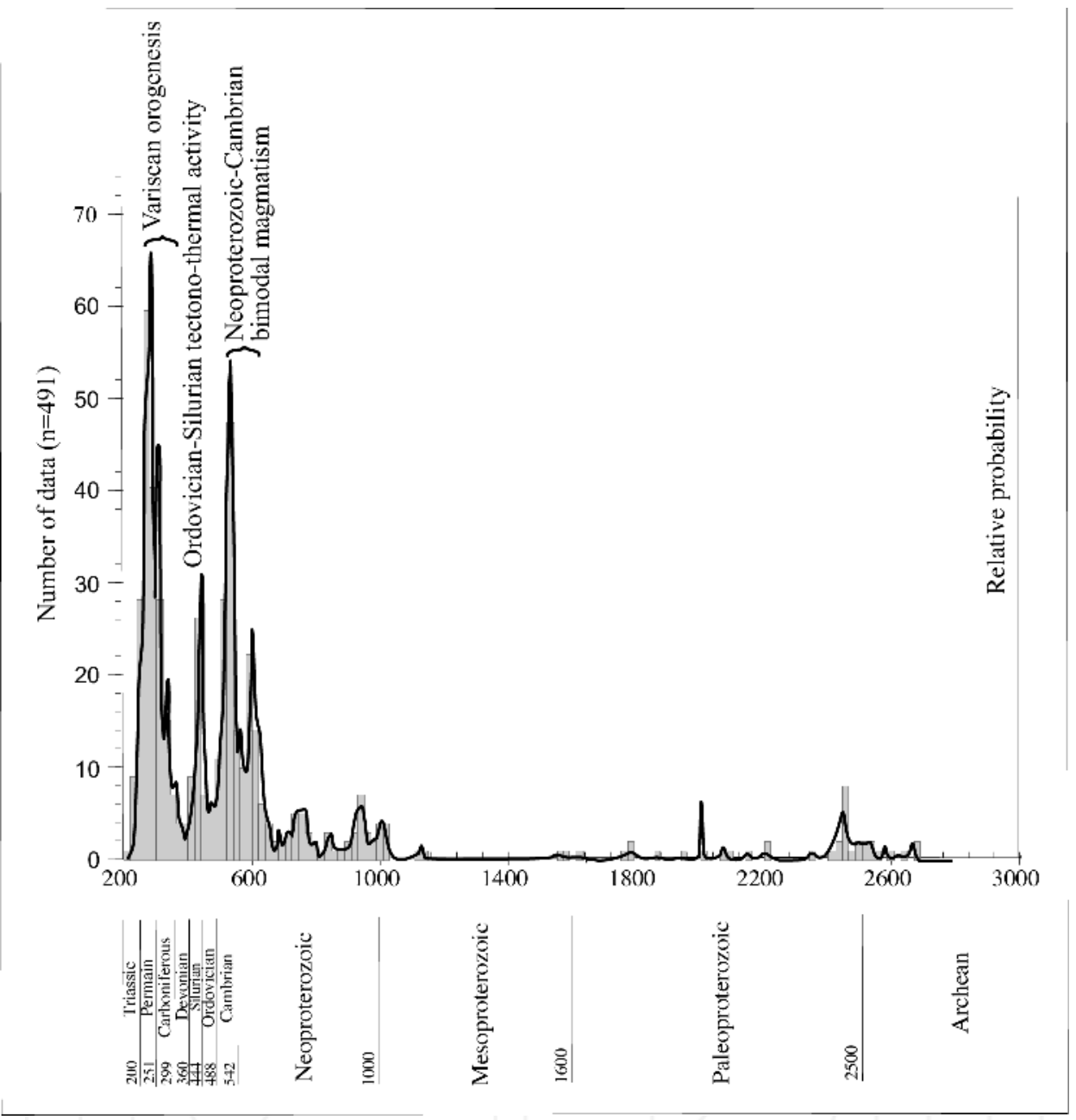

Figure 11. Histogram and probability density curve for U-Pb concordant data on zircon ( $n=491)$ from CPT (Tab.1). References: [7, 15-24].

\section{Author details}

Annamaria Fornelli*, Giuseppe Piccarreta and Francesca Micheletti

*Address all correspondence to: annamaria.fornelli@uniba.it

Dip. di Scienze della Terra e Geoambientali, Università degli Studi di Bari Aldo Moro, Italy 


\section{References}

[1] Scherer E E, Whitehouse M J and Münker C. Zircon as a Monitor of Crustal Growth. Elements 2007; 3(1) 19-24.

[2] Cocherie A and Robert M. Laser ablation coupled with ICP-MS applied to U-Pb zircon geochronology: A review of recent advances. Gondwana Research 2008; 14, 597608.

[3] Ru-Xiong L, Chang-Zhi W, Lian-Xing G, Zun-Zhong Z, Guo-Xiang C, Yao-Hui J. Zircon $\mathrm{U}-\mathrm{Pb}$ chronology and $\mathrm{Hf}$ isotope of the Xingxingxia granodiorite from the Central Tianshan zone (NW China): Implications for the tectonic evolution of the southern Altaids. Gondwana Research 2011; 20 582-593.

[4] Ireland TR and Williams IS., 2003. Considerations in zircon geochronology by SIMS. In: Hanchar JM and Hoskin PWO (ed.) Zircon, Reviews in Mineralogy \& Geochemistry; 2003. (53), 215-241.

[5] Kosler J and Sylvester PJ. Present trends and the future of zircon in geochronology: laser ablation ICPMS. In: Hanchar JM and Hoskin PWO (ed.) Zircon, Reviews in Mineralogy \& Geochemistry; 2003. (53), 243-275.

[6] Corfu F, Hanchar JM, Hoskin PWO, Kinny P. Atlas of zircon textures. In: Hanchar JM and Hoskin PWO (ed.) Zircon, Reviews in Mineralogy \& Geochemistry; 2003. (53), 468-500.

[7] Fornelli A, Langone A, Micheletti F, Piccarreta G. Time and duration of Variscan high-temperature metamorphic processes in the south European Variscides. Constraints from $\mathrm{U}-\mathrm{Pb}$ chronology and trace - element chemistry of zircon. Mineralogy and Petrology 2011; 103, 101-122.

[8] Rubatto D. Zircon trace element geochemistry: partitioning with garnet and the link between U-Pb ages and metamorphism. Chemical Geology 2002; 184,123-138.

[9] Moller A, O’Brien PJ, Kennedy A, Kroner A. Polyphase zircon in ultrahigh-temperature granulites (Rogaland, SW Norway): constraints for $\mathrm{Pb}$ diffusion in zircon. Journal of Metamorphic Geology 2002; 20, 727-740.

[10] $\mathrm{Wu} \mathrm{Y}$ and Zheng Y. Genesis of zircon and its constraints on interpretation of U-Pb age. Chinese Science Bulletin 2004; 49(15), 1554-1569.

[11] Harley S and Kelly NM. The impact of zircon - garnet REE distribution data on the interpretation of zircon $\mathrm{U}-\mathrm{Pb}$ ages in complex high - grade terrains: an example from the Rauer Islands, east Antarctica. Chemical Geology 2007; 241, 62-87.

[12] Nehring F, Foley SF, Hölttä P, Van Den Kerkhof. Internal differentiation of the Archean continental crust: fluid-controlled partial melting of granulites and TTG-amphibolite associations in central Finland. Journal of Petrology 2009; 50(1), 3-35. 
[13] Xia X, Sun M, Geng H, Sun Y, Wang Y, Zhao G. Quasi-simultaneous determination of $\mathrm{U}-\mathrm{Pb}$ and $\mathrm{Hf}$ isotope compositions of zircon by excimer laser-ablation multiplecollector ICPMS. Journal of Analitycal Atomic Spectrometry 2011; 26, 1868-1871.

[14] Zhou LG, Xia XQ, Zheng YF, Chen RX. Multistage growth of garnet in ultrahighpressure eclogite during continental collision in the Dabie orogen: constrained by trace elements and U-Pb ages. Lithos 2011; 127, 101-127.

[15] Trombetta A, Cirrincione R, Corfu F, Mazzoleni P, Pezzino A. Mid-Ordovician $\mathrm{U}^{\wedge} \mathrm{Pb}$ ages of porphyroids in the Peloritan Mountains (NE Sicily): paleogeographic implications for the evolution of the Alboran microplate. Journal of the Geological Society, London 2004; 161, 1-13.

[16] Micheletti F, Barbey P, Fornelli A, Piccarreta G, Deloule E. Latest Precambrian to Early Cambrian $\mathrm{U}-\mathrm{Pb}$ zircon ages of augen gneisses from Calabria (Italy), with inference to the Alboran microplate in the evolution of the peri-Gondwana terranes. International Journal of Earth Sciences 2007; 96(5) 843-860.

[17] Micheletti F, Fornelli A, Piccarreta G, Barbey P, Tiepolo M. The basement of Calabria (southern Italy) within the context of the Southern European Variscides: LA-ICPMS and SIMS U-Pb zircon study. Lithos 2008; 104, 1-11.

[18] Micheletti F, Fornelli A, Piccarreta G, Tiepolo M. U-Pb zircon data of Variscan metaigneous acidic rocks from an Alpine shear zone in Calabria (southern Italy). International Journal of Earth Sciences 2011; 100(1), 139-155.

[19] Fiannacca P, Williams IS, Cirrincione R, Pezzino A. Crustal Contributions to Late Hercynian Peraluminous Magmatism in the Southern Calabria Peloritani Orogen, Southern Italy: Petrogenetic Inferences and the Gondwana Connection. Journal of Petrology 2008; 49, 1897-1514.

[20] Langone A. Herynian low-pressure metamorphism: tectono-thgermal evolution of the Mandatoriccio complex (Sila Massif, Calabria). PhD thesis. Università di Bologna (Italy); 2008.

[21] Grande A. Evoluzione tettono-metamorfica delle metamorfiti affioranti a Palizzi Marina (Massiccio dell'Aspromonte, Calabria). PhD thesis. Università degli Studi di Bari Aldo Moro (Italy); 2009.

[22] Fornelli A, Langone A, Micheletti F, Pascazio A, Piccarreta G. The role of trace element partitioning between garnet, zircon and orthopyroxene on the interpretation of zircon U-Pb ages: an example from high-grade basement in Calabria (Southern Italy). International Journal of Earth Sciences 2014; 103(2), 487-507.

[23] Williams IS, Fiannacca P, Cirrincione R, Pezzino A. Peri-Gondwanian origin and early geodynamic history of NE Sicily: A zircon tale from the basement of the Peloritani Mountains. Gondwana Research 2012; 22, 855-865. 
[24] Muschitiello A. Geochimica, geochimica isotopica ed età U-Pb su zirconi dei metagabbri e delle metabasiti nella crosta profonda ercinica delle Serre (Calabria). Università degli Studi di Bari Aldo Moro (Italy); 2012.

[25] Griffin BJ, Joy DC, Michael JR. A Comparison of a Luminescence-Based VPSE and an Electron-Based GSED for SE and CL Imaging in Variable Pressure SEM with Conventional SE Imaging. Microscopy and Microanalysis 2010; 16(2), 624-625.

[26] Acquafredda P and Fiore S. La microscopia elettronica a scansione. In "Argille e minerali delle argille" 2005; (V), 143-188.

[27] Gerdes A. and Zeh A. Combined U-Pb and Hf isotope LA-(MC-)ICP-MS analyses of detrital zircons: Comparison with SHRIMP and new constraints for the provenance and age of an Armorican metasediment in Central Germany. Earth and Planetary Science Letters 2006; 249(1-2), 247-61.

[28] Horn I, Rudnick RL, Mc Donough WF. Precise elemental and isotope ratio determination by simultaneous solution nebulization and laser ablation-ICP-MS: application to U-Pb geochronology. Chemical Geology 2000; 164, 281-301.

[29] Tiepolo M. In situ Pb geochronology of zircon with laser ablation-inductively coupled plasma-sector field mass spectrometry. Chemical Geology 2003; 199, 159-177.

[30] Wiedenbeck M, Allé P, Corfu F, Griffin WL, Meier M, Oberli F, Von Quadt A, Roddick JC, Spiegel W. Three natural zircon standards for U-Th-Pb, Lu-Hf, trace elements and REE analyses. Geostandard Newletters 1995; 19, 1-23.

[31] Ketchum JWF, Jackson SE, Culshaw NG, Barr SM. Depositional and tectonic setting of the Paleoproterozoic Lower Aillik Group, Makkovik Province, Canada: evolution of a passive margin - foredeep sequence based on petrochemistry and U-Pb (TIMS and LAM-ICP-MS) geochronology. Precambrian Research 2001; 105, 331- 356.

[32] van Achterbergh E, Ryan CG, Jackson S, GriffinW. Data reduction software for LAICPMS. In: Sylvester P (ed.) Laser ablation ICPMS in the earth science. Mineralogy Association Canada 2001; 29, 239-24.

[33] Horstwood MSA, Foster GL, Parrish RR, Noble SR, Nowell GM. Common-Pb corrected in situ U-Pb accessory mineral geochronology by LA-MC-ICP-MS. Journal of Analytical Atomic Spectrometry 2003; 18, 837-846.

[34] Compston W, Williams IS, Meyer C. U-Pb geochronology of zircons from lunar breccia 73217 using a sensitive high mass-resolution ion microprobe. Journal of geofisical research: Solid Earth 1984; 89(S02), B525-B534.

[35] Stacey JS and Kramers JD. Approximation of terrestrial lead isotope evolution by a two-stage model. Earth and Planetary Science Letters 1975; 26, 207-221. 
[36] Deloule E, Alexandrov P, Cheilletz A, Laumonier B, Barbey P. In situ U/Pb zircon ages for Early Ordovician magmatism in the eastern Pyrenees, France: the Canigou orthogneisses. International Journal of Earth Sciences 2002; 91, 398-405.

[37] Williams I S and Claesson S. Isotopic evidence for the Precambrian provenance and Caledonian metamorphism of high grade paragneisses from the Seve Nappes, Scandinavian Caledonides: II. Ion microprobe zircon U-Th-Pb. Contributions to Mineralogy and Petrology 1987; 97, 205-217.

[38] Claoue.-Long JC, Compston W, Roberts J, Fanning CM. Two Carboniferous ages: a comparison of SHRIMP zircon dating with conventional zircon ages and 40Ar/39Ar analysis. In: Berggren WA, Kent DV, Aubrey MP, Hardenbol J. (ed.) Geochronology, Time Scales and Global Stratigraphic Correlation. Society of Economic Paleontologists and Mineralogists 1995, Special Publication 54, p3-21.

[39] Cumming GL and Richards JR. Ore lead isotope ratios in a continuously changing Earth. Earth and Planetary Science Letters 1975; 28, 155-171.

[40] Steiger RH and Jager E. Subcommission on geochronology: convention on the use of decay constants in geo-and cosmochronology. Earth and Planetary Science Letters 1977; 36, 359-362.

[41] Ludwig KR. User's Manual for A Geochronological Toolkit for Microsoft Excel. Berkeley Geochronology Center, Spec Publ No. 4; 2003.

[42] Bonardi G, Cavazza W, Perrone V, Rossi S. Calabria-Peloritani terrane and northern Ionian Sea. In: Vai GB, Martini IP (ed.) Anatomy of an Orogen: the Apennines and Adjacent Mediterranean Basins. Kluwer Academic Publishers; 2001. p 287-306.

[43] Festa V, Messina A, Paglionico A, Piccarreta G, Rottura A. Pre-Triassic history recorded in the Calabria-Peloritani segment of the Alpine chain, southern Italy. An overview. Spec Iss 2: A showcase of the Italian research in metamorphic petrology. Periodico di Mineralogia 2004; 73, 57-71.

[44] Acquafredda P, Lorenzoni S, Zanettin-Lorenzoni E. La sequenza Paleozoica dell'Unità di Bocchigliero (Sila, Calabria). Rendiconti Società Geologica Italiana 1988; 11, 5-22.

[45] Acquafredda P, Barbieri M, Lorenzoni S, Zanettin-Lorenzoni E. The age of volcanism and metamorphism of the Bocchigliero Paleozoic sequence (Sila - southern Italy). Rendiconti Accademia dei Lincei 1991; 9, 145-156.

[46] Langone A, Godard G, Prosser G, Caggianelli A, Rottura A, Tiepolo M. P-T-t path of the Variscan low-pressure rocks from the Mandatoriccio complex (Sila Massif, Calabria, Italy): new insights for crustal evolution. Journal of Metamorphic Geology 2010; 28,137-162.

[47] Lorenzoni S and Zanettin-Lorenzoni E. Note illustrative della carta geologica della Sila alla scala 1:200.000. Memorie della Società Geologica Italiana 1983; 36, 317-342. 
[48] Borghi A, Colonna V. Compagnoni R. Structural and metamorphic evolution of the Bocchigliero and the Mandatoriccio Complex in the Sila nappe (Calabrian-Peloritan Arc, Southern Italy). IGCP n²76, Newsletters 1992; 5, 321-334.

[49] Colonna V and Piccarreta G. Contributo alla conoscenza dell’Unità di Castagna in Sila Piccola: rapporti tra micascisti, paragneiss e gneiss occhiadini. Bollettino Società Geologica Italiana 1976; 95, 39-48.

[50] Paglionico A and Piccarreta G. Le Unità del Fiume Pomo e di Castagna nelle Serre Settentrionali (Calabria). Bollettino Società Geologica Italiana 1976; 95, $27-37$.

[51] Caggianelli A, Prosser G, Del Moro A. Cooling and exhumation history of deep-seated and shallow level, late Hercynian granitoids from Calabria. Geological Journal $2000 ; 35,33-42$.

[52] Schenk $\mathrm{V}$. U-Pb and $\mathrm{Rb}-\mathrm{Sr}$ radiometric dates and their correlation with metamorphic events in the granulite-facies basement of the Serre, Southern Calabria (Italy). Contribution to Mineralogy and Petrology 1980; 73,23-38.

[53] Fiannacca P, Williams IS, Cirrincione R, Pezzino A. The augen gneisses of the Peloritani Mountains (NE Sicily): Granitoid magma production during rapid evolution of the northern Gondwana margin at the end of the Precambrian. Gondwana Research 2013; 23(2), 782-796.

[54] D’Amico C, Rottura A, Maccarrone E, Puglisi G. Peraluminous granitic suite of Calabria-Peloritani arc (Southern Italy). Rendiconti Società Italiana Mineralogia e Petrologia 1982; 38, 35-52.

[55] Rottura A, Bargossi GM, Caironi V, Del Moro A, Maccarrone E, Macera P, Paglionico A, Petrini R, Piccareta G, Poli G. Petrogenesis of contrasting Hercynian granitoids from the Calabrian Arc, Southern Italy. Lithos 1990; 24, 97-119.

[56] Rottura A, Caggianelli A, Campana R, Del Moro A. Petrogenesis of Hercynian peraluminous granites from the Calabrian Arc, Italy. European Journal of Mineralogy $1993 ; 5,737-754$.

[57] Fiannacca P, Brotzu P, Cirrincione R, Mazzoleni P, Pezzino A. Alkali metasomatism as a process for trondhjemite genesis: evidence from Aspromonte Unit, north-eastern Peloritani, Sicilyan Mineralogy and Petrology, 2005(a); 84, 19-45.

[58] Ortolano G, Cirrincione R, Pezzino A. P-T evolution of alpine metamorphism in the southern Aspromonte Massif (Calabria-Italy). Schweizer Mineralogische und Petrographische Mitteilungen 2005; 85(1), 31-56.

[59] Cirrincione R, Fazio E, Fiannacca P, Ortolano G, Pezzino A, Punturo R. Petrological and microstructural constraints for orogenetic exhumation modelling of HP rocks: The example of southern Calabria Peloritani Orogen (Western Mediterranean), in GeoMod 2008, Third International Geomodelling Conference, Firenze, 22-24 September 2008. Bollettino Geofisica Teorica e Applicata 2008; 49(2), 141-146. 
[60] Schenk V and Todt W. Age of formation of the southern Calabrian crust. Terra Abstracts $1989 ; 1,350$.

[61] Schenk V. The exposed crustal cross section of southern Calabria, Italy: structure and evolution of a segment of Hercynian crust. In: Salisbury MH, Fountain DM (ed.) Exposed Cross-sections of the Continental Crust. Kluwer Academic Publisher, Netherlands; 1990. p.21-42.

[62] Cahen L, Snelling NJ, Delhal J, Vail JR. The Geochronology and Evolution of Africa. Clarendon Press, Oxford; 1984.

[63] Mallard LD, Rogers JJW. Relationship of Avalonian and Cadomian terranes to Grenville and Pan-African events. Journal of Geodynamics 1997; 23,197-221.

[64] [64] Zeck HP and Williams IS. Inherited and Magmatic Zircon from Neogene Hoyazo Cordierite Dacite, SE Spain-Anatectic Source Rock Provenance and Magmatic Evolution. Journal of Petrology 2002; 43 (6), 1089-1104

[65] [65] Samson SD, D'Lemos RS, Miller BV, Hamilton MA. Neoproterozoic paleogeography of the Cadomia and Avalon terranes: constraints from detrital zircon $\mathrm{U}-\mathrm{Pb}$ ages. Journal of Geological Society of London 2005;162,65-71.

[66] [66] Murphy J B, Gutierrez-Alonso G, Nance R D, Fernandez-Suarez J, Keppie J D, Quesada C, Strachan R A, Dostal J. Origin of the Rheic Ocean: Rifting along a Neoproterozoic suture? Geological Society of America 2006; 34(5), 325-328.

[67] [67] Stampfli G.M., von Raumer J. and Wilhem C. The distribution of Gondwana-derived terranes in the Early Paleozoic. J.C. Gutiérrez-Marco, I. Rábano and D. GarcíaBellido (ed.), Ordovician of the World; 2011. p.567-574. ISBN 978-84-7840-857-3.

[68] [68] von Raumer JF, Bussy F, Schaltegger U, Schulz B, Stampfli GM. Pre-Mesozoic Alpine basements - Their place in the European Paleozoic framework. GSA Bulletin 2013; 125 (1-2), 89-108.

[69] [69] Hanchar JM, Miller CF. Zircon zonation patterns as revealed by cathodoluminescence and backscattered electron images: implications for interpretation of complex crustal histories. Chemical Geology 1993; 110, 1-13.

[70] [70] Duchene S, Fornelli A, Micheletti F, Piccarreta G. Sm-Nd chronology of porphyroblastic garnets from granulite facies metabasic rocks in Calabria (Southern Italy): inferences for preserved isotopic memory and resetting. Mineralogy and Petrology 2013; 107(4), 539-551.

[71] Neubauer F. Evolution of late Neoproterozoic to early Paleozoic tectonic elements in Central and Southeast European Alpine mountain belts: review and synthesis. Tectonophysics 2002; 352, 87-103.

[72] Fornelli A, Langone A, Micheletti F, Piccarreta G. Application of U-Pb dating and chemistry of zircon in the continental crust of Calabria (Southern Italy). In: Van Dijk 
G, Van den Berg V (ed.) Zircon and Olivine: Characteristics, Types and Uses; 2012. ebook ISBN: 978-1-62100-990-0

[73] Rubatto D and Hermann J. Experimental zircon/melt and zircon/ garnet trace element partitioning and implications for the geochronology of crustal rocks. Chemical Geology 2007; 241, 62-87.

[74] Xia XQ, Zheng YF, Yuan H, Wu FY. Contrasting Lu-Hf and U-Th-Pb isotope systematics between metamorphic growth and recrystallization of zircon from eclogitefacies metagranites in the Dabie orogen, China. Lithos 2009; 112(3-4),477-496.

[75] Fornelli A, Micheletti F, Piccarreta G. The Neoproterozoic-Early Cambrian felsic magmatism in Calabria (Italy): inferences as to the origin and geodynamic setting. Periodico di Mineralogia 2007; SPECIAL ISSUE 76, 99-112.

[76] Fiannacca P, Williams I, Cirrincione R, Pezzino A. Post-collisional granitoid magmatism in the Calabria-Peloritani Terrane during the final stages of north Gondwana assembly. Geoitalia 2011, 19-23 September 2011, Torino, Italy. Epitome 04.0632.

[77] Thomas RJ, Chevallier LP, Gresse PG, Harmer RE, Eglington BM, Armstrong RA, de Beer $\mathrm{CH}$, Martini JEJ, de Kock GS, Macey PH, Ingram BA. Precambrian evolution of the Sirwa Window, Anti-Atlas Orogen, Morocco. Precambrian Research 2002; 118, 157.

[78] Walsh GJ, Aleinikoff J, Benziane F, Yazidi A, Armstrong TR. U-Pb zircon geochronology of the Palaeoproterozoic Tagragra de Tata inlier and its Neoproterozoic cover, western Anti-Atlas, Morocco. Precambrian Research 2002; 117, 1-20.

[79] Acef K, Liégeois JP, Ouabadi A, Latouche L. The Anfeg post-collisional Pan-African high-K calc-alkaline batholith (Central Hoggar, Algeria), result of the LATEA microcontinent metacratonisation. Journal of African Earth Sciences 2003; 37, 295-311.

[80] Azzouni-Sekkal A, Liégeois JP, Bechiri-Benmerzoug F, Belaidi-Zinet S, Bonin B. The "Taourirt" magmatic province, a marker of the closing stage of the Pan-African orogeny in the Tuareg Shield: review of available data and Sr-Nd isotope evidence. Journal of African Earth Sciences 2003; 37, 31-350.

[81] Lahondère D, Thiéblemont D, Goujou JC, Le Métour J, Barbey P, Bronner G, Deschamps M, Cocherie A, Guerrot C, Marchand J. Evolution archéenne à paléoprotérozoïque de la Dorsale de Rgueïbat (Nord de la Mauritanie). 20th Coll Afr Geol, Orléans, 2004. Abstract, p 251.

[82] Stedra V, Kachlik V, Kryza, R. Coronitic metagabbros of the Mariánské Lázně Complex and Teplá Crystalline Unit: inferences for the tectonometamorphic evolution of the western margin of the Teplá-Barrandian Unit, Bohemian Massif. Geological Society, London, Special Publications 2002; 201, 217-236.

[83] Gasquet D, Levresse G, Cheilletz A, Azizi-Samir MR, Mouttaqi A. Contribution to a geodynamic resconstruction of the Anti-Atlas (Morocco) during Pan-African times 
with the emphasis on inversion tectonics and metallogenic activity at the Precambrian-Cambrian transition. Precambrian Research 2005; 140,157-182.

[84] Vavra G, Gebauer D, Schmid R, Compton W. Multiple zircon growth and recrystallization during polyphase Late Carboniferous to Triassic metamorphism in granulite of Ivrea Zone (Southern Alps): an ion microprobe (SHRIMP) study. Contrib Mineral Petrol 1996; 122, 337-358.

[85] Vavra G, Schmid R, Gebauer D. Internal morphology, habit and U-Th-Pb microanalysis of amphibolite-to-granulite facies zircons: geochronology of the Ivrea Zone (Southern Alps). Contribution to Mineralogy and Petrology 1999; 134, 380-404.

[86] Schenk V. P-T-t path of the lower crust in the Hercynian fold belt of southern Calabria. In: Daly JS, Cliff RA, Yardley BWD (ed.) Evolution of metamorphic belts. Geological Society Special Publication 1989. 43, 337-342.

[87] Faure, M. Microtéctonique et charriage Est-Ouest des nappes alpines profondes de Sila (Calabre, Italie méridionale). Revue de Géologie Dynamique et Géographie Physique 1980; 22(2), 135-146.

[88] Amodio Morelli L, Bonardi G, Colonna V, Dietrich D, Giunta G, Ippolito F, Liguori V, Lorenzoni S, Paglionico A, Perrone V, Piccarreta G, Russo M, Scandone P, Zanettin Lorenzoni E, Zuppetta A. L'arco Calabro-Peloritano nell'orogene AppenninicoMaghrebide. Memorie Società Geologica Italiana 1976; 17, 1-60.

[89] Del Moro A, Fornelli A, Piccarreta G. Tectonothermal history of the Hercynian continental crust of the Serre (southern Calabria, Italy) monitored by $\mathrm{Rb} / \mathrm{Sr}$ biotite resetting. Terra Nova 2000; 12, 239-244.

[90] Lee JKW, Williams IS, Ellis DJ. Determination of $\mathrm{Pb}, \mathrm{U}$ and $\mathrm{Pb}$ diffusion rates in zircon. Abstract, GAC/MAC Annual Meeting, Ottawa 1997 A87.

[91] Cherniak DJ, Hanchar JM, Watson EB. Rare-earth diffusion in zircon. Chemical Geology 1997; 134, 289-301.

[92] Cherniak DJ and Watson EB. Pb diffusion in zircon. Chemical Geology 2000; 172, 5-24.

[93] Angì G, Cirrincione R, Fazio E, Fiannacca P, Ortolano G, Pezzino A. Metamorphic evolution of preserved Variscan upper crust in the Alpine Calabria-Peloritani Orogen (southern Italy): structural and petrological constraints from the Serre Massif metapelites. Lithos 2010; 115, 237-262.

[94] Geisler T, Schaltegger U, Tomaschek F. Re-equilibration of zircon in aqueous fluids and melts. Elements 2007; 3(1), 43-50.

[95] Fornelli A, Pascazio A, Piccarreta G. Diachronic and different metamorphic evolution in the fossil Variscan lower crust of Calabria. International Journal of Earth Sciences 2012(b); 101(5),1191-1207. 
[96] Caggianelli A and Prosser G. Modelling the thermal perturbation of the continental crust after intraplating of thick granitoid sheets: a comparison with the crustal sections in Calabria (Italy). Geological Magazine 2002; 139(6), 699-706.

[97] Handy MR and Zingg A. The tectonic and rheological evolution of an attenuated cross section of the continental crust: Ivrea crustal section, southern Alps, northwestern Italy and southern Switzerland. Geological Society of America Bulletin 1991; 103, 236-253.

[98] Gardien V, Lardeaux JM, Ledru P, Allemand P, Guillot S. Metamorphism during late orogenic extension: insights from the French Variscan belt. Bulletin Société Géologique France 1997; 168, 271-286.

[99] Lardeaux J, Ledru P, Daniel I, Duchene S. The Variscan French Massif Central-a new addition to the ultra-high pressure metamorphic 'club': exhumation processes and geodynamic consequences. Tectonophysics 2001; 332, 143-167.

[100] Rossi P, Cocheri A, Fanning CM, Deloule E. Variscan to eo-Alpine events recorded in European lower-crust zircons sampled from the French Massif Central and Corsica, France. Lithos 2006; 87,235-260.

[101] Giacomini F, Bomparola RM, Grezzo C, Guldbransen H. The geodynamic evolution of the Southern European Variscides: constraints from the $\mathrm{U} / \mathrm{Pb}$ geochronology and geochemistry of the Palaeozoic magmatic-sedimentary sequences of Sardinia (Italy). Contribution to Mineralogy and Petrology 2006; 152,19-42.

[102] Lu M, Hofmann AW, Mazzucchelli M, Rivalenti G. The maficultramafic complex near Finero (Ivrea-Verbano Zone). II. Geochronology and isotope geochemistry. Chemical Geology 1997; 140, 223-235.

[103] Peressini G, Quick JE, Sinigoi S, Hofmann AW, Fanning M. Duration of a large mafic intrusion and heat transfer in the lower crust: a SHRIMP U-Pb zircon study in the Ivrea-Verbano Zone (Western Alps, Italy). Journal of Petrology 2007; 48, 1185-1218.

[104] Schaltegger U and Gebauer D. Pre-Alpine geochronology of the Central, Western and Southern Alps. Schweiz Mineral Petrogr Mitteilungen 1999; 79, $79-87$.

[105] Katayama I, Maruyama S, Parkinson CD, Terada K, Sano Y. Ion micro-probe U-Pb zircon geochronology of peak and retrograde stages of ultrahigh-pressure metamorphic rocks from the Kokchetav massif, northern Kazakhstan. Earth and Planetary Science Letters 2001; 188(1-2), 185-198.

[106] Hermann J, Rubatto D, Korsakov A, Shatsky VS. Multiple zircon growth during fast exhumation of diamondiferous, deeply subducted continental crust (Kokchetav Massif, Kazakhstan). Contributions to Mineralogy and Petrology 2001; 141(1), 66-82.

[107] Moller A, O’Brien PJ, Kennedy A, Kroner A. Polyphase zircon in ultrahigh-temperature granulites (Rogaland, SW Norway): constraints for $\mathrm{Pb}$ diffusion in zircon. Journal of Metamorphic Geology 2002; 20, 727-740. 
[108] $\mathrm{Wu} \mathrm{Y}$ and Zheng Y. Genesis of zircon and its constraints on interpretation of U-Pb age. Chinese Science Bulletin 2004; 49, 1554-1569.

[109] Liati A. Identification of repeated Alpine (ultra) high-pressure metamorphic events by U-Pb SHRIMP geochronology and REE geochemistry of zircon: the Rhodope zone of Northern Greece. Contributions to Mineralogy and Petrology 2005; 150, 608-630.

[110] Liu Y, Gao S, Hu Z, Gao C, Zong K, Wang D. Continental and oceanic crust recycling-induced melt-peridotite interactions in the Trans-North China Orogen: $\mathrm{U}-\mathrm{Pb}$ dating, Hf isotopes and trace elements in zircons from mantle xenoliths. Journal of Petrology 2010; 51(1-2), 537-571.

[111] McClelland WC, Power SE, Gilotti JA, Mazdab FK, Wopenka B. U-Pb SHRIMP geochronology and trace-element geochemistry of coesite-bearing zircons, North-East Greenland Caledonides. Geological Society of America, Special Paper 403, Ultrahighpressure Metamorphism: Deep Continental Subduction, 2006.

[112] Mcclelland WC, Gilotti JA, Mazdab FK, Wooden JL. Trace-element record in zircons during exhumation from UHP conditions, North-East Greenland Caledonides. European Journal of Mineralogy 2009; 21, 1135-1148.

[113] Chen RX, Zheng YF, Xie L. Metamorphic growth and recrystallization of zircon: distinction by simultaneous in situ analyses of trace elements, U-Th-Pb and Lu-Hf isotopes in zircons from eclogite-facies rocks in the Sulu orogen. Lithos 2010; 114, 132154.
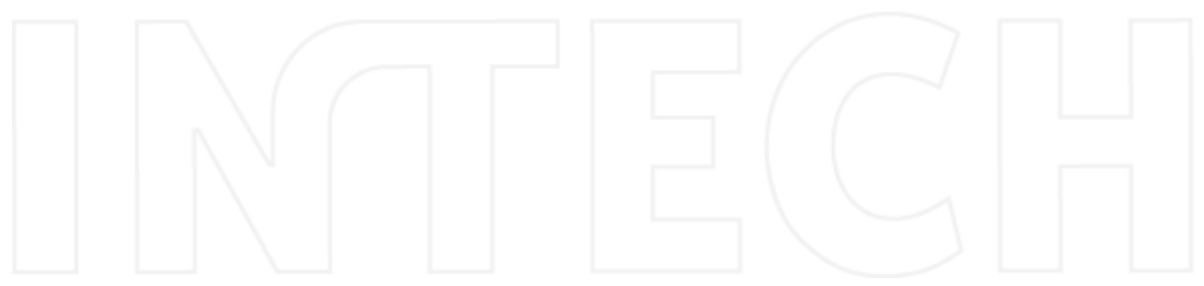

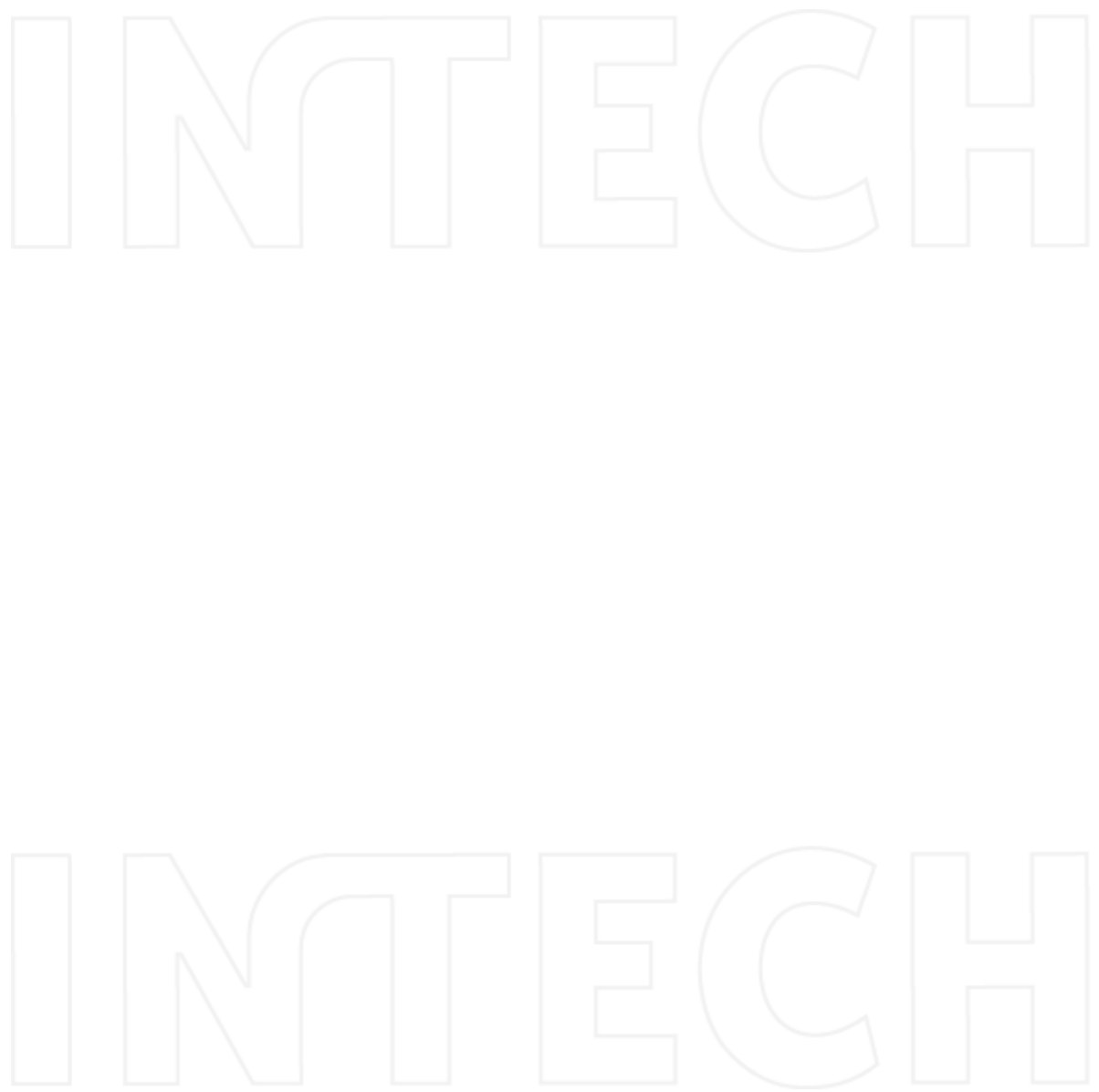


\title{
Layered Pge Paleoproterozoic (LIP) Intrusions in the N-E Part of the Fennoscandian Shield - Isotope Nd-Sr and ${ }^{3} \mathrm{He} /{ }^{4} \mathrm{He}$ Data, Summarizing U-Pb Ages (on Baddeleyite and Zircon), Sm-Nd Data (on Rock-Forming and Sulphide Minerals), Duration and Mineralization
}

\author{
T. Bayanova, F. Mitrofanov, P. Serov, L. Nerovich, \\ N. Yekimova, E. Nitkina and I. Kamensky
}

Additional information is available at the end of the chapter

http://dx.doi.org/10.5772/58835

\section{Introduction}

There are about 20 Palaeoproterozoic layered mafic-ultramafic bodies in Finland, most of which occur in a roughly east-west-trending, $300 \mathrm{kmlong}$ belt known as the TornioNa.ra.nka.vaara Belt (Alapieti et al. 1990; Vogel et al. 1998; Iljina \& Hanski 2005). The belt (Fig. 1) extends for a few kilometres into Sweden (Tornio intrusion), and for several tens of kilometres into the Russian Karelia (Olanga complex). Together the intrusions make up the Southern, or Fenno-Karelian Belt, FKB (Mitrofanov et al. 1997).

In the NE of the province, the Northern, or Kola Belt (KB) strikes northwestwards for about $500 \mathrm{~km}$ (Fig. 1). It includes more than ten isolated layered mafic-ultramafic bodies that are mostly ore-bearing (Mitrofanov et al. 1997). The central part of the Kola Belt has been suggested to be part of a triple junction typical of intraplate rifting (Pirajno 2007) and is occupied by the Monchegorsk Layered Complex with a fairly complete range of ore types $(\mathrm{Cr}, \mathrm{Cu}, \mathrm{Ni}, \mathrm{Co}, \mathrm{Ti}$, $\mathrm{V}, \mathrm{Pt}, \mathrm{Pd}, \mathrm{Rh})$. The western and eastern arms of the triple junction are composed of large anorthosite-troctolite (Main Ridge, Pyrshin, Kolvitsa) intrusions (Fig. 1). The most typical PGEbearing layered pyroxenite-norite-gabbroanorthosite intrusions of the Kola Belt (e.g. Mt Generalskaya, Monchegorsk Layered Complex, Fedorovo-Pansky) are confined to boundaries between early Proterozoic rifts which were in-filled with volcano-sedimentary rocks overlying the Archaean basement (Schissel et al. 2002; Mitrofanov et al. 2005). In these cases, similar to 
those in Finland, the intrusive rocks underwent relatively low-grade local metamorphism and preserve cumulus and intracumulus minerals.

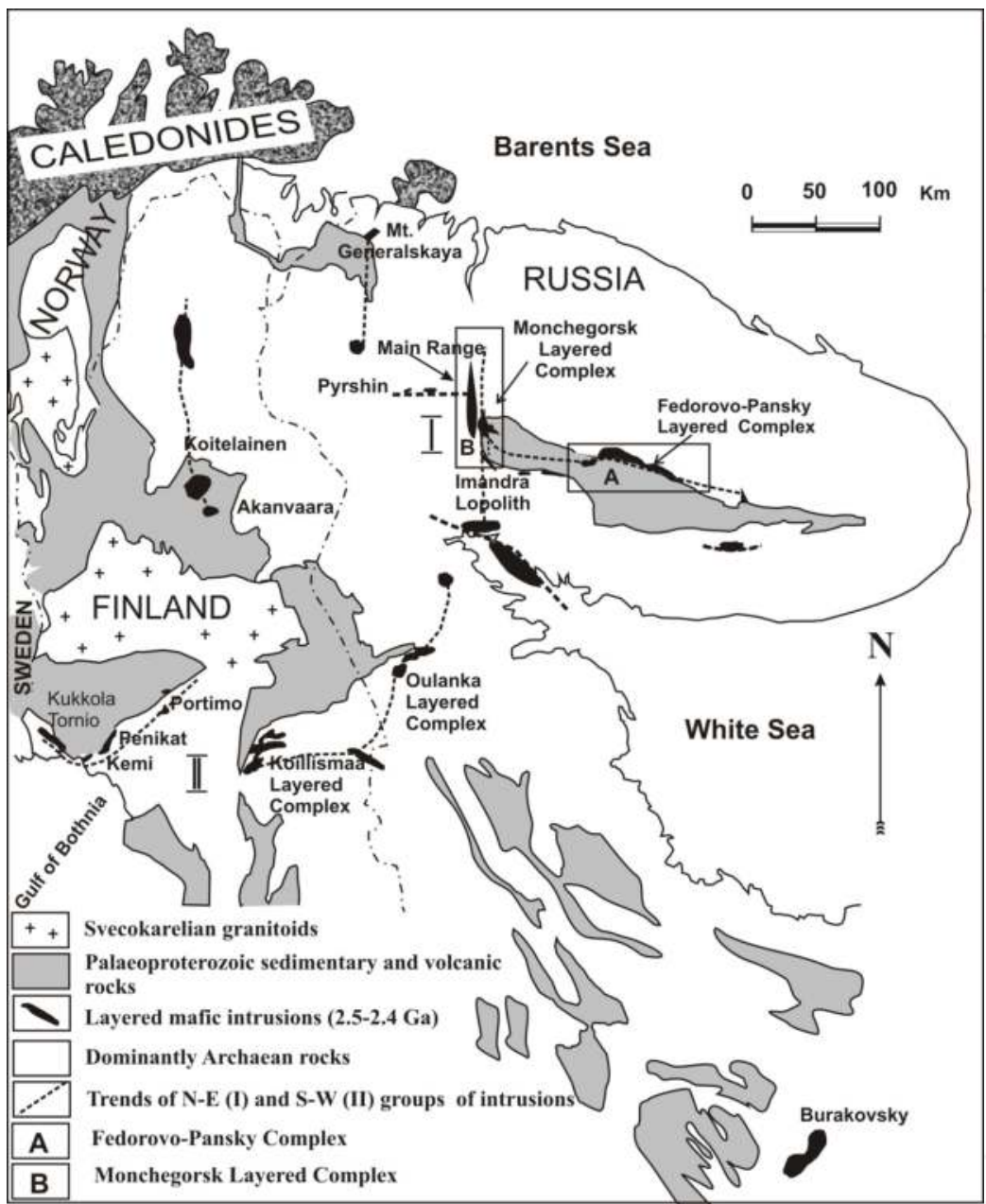

Figure 1. Generalized geological map of the northeastern part of the Baltic Shield and the location of Paleoproterozoic mafic layered intrusions (Mitrofanov et al., 2005).

Convincing arguments in support of the mantle plume hypothesis, either as 'shallow plumes' (from c. $670 \mathrm{~km}$ ) or 'deep plumes' (from the core - mantle boundary) have been put forward for relatively young well-preserved Palaeozoic and recent large igneous provinces (LIPs) (Coffin \& Eldhom 1994; Heaman 1997; Ernst \& Buchan 2003; French et al. 2008). Voluminous magmatism is considered to be related to mantle plumes that occurred throughout the Precambrian (Condie 2001; Pirajno 2007). The best records of a plume source are Cu, PGE, Ti, 
$\mathrm{V}$ and Fe. All the above-mentioned rock types, metals, host rift-associated volcanic rocks and mafic dykes are found in the relatively recently described East Scandinavian Palaeoproterozoic Large Igneous Province (LIP) (Iljina \& Hanski 2005) with a total area of more than $200000 \mathrm{~km} 2$ (Fig. 1). In Finland, these geological complexes have been widely studied (Alapieti et al. 1990; Huhma et al. 1990; Vogel et al. 1998; Hanski et al. 2001) and the data were su $\mu$ marized by Iljina \& Hanski (2005). Only a few publications on similar Russian complexes of the Baltic Shield have been written or translated into English (Papunen \& Gorbunov et al. 1985; Balashov et al. 1993; Bayanova \& Balashov 1995; Bayanova 2009; Amelin et al. 1995; Sharkov et al. 1995; Mitrofanov et al. 1997; Mitrofanov \& Bayanova 1999; Chashchin et al. 2002; Schissel et al. 2002; Mitrofanov et al. 2002, 2005).

This chapter presents a brief geological description of the Russian mafic-ultramafic intrusions of the Baltic Shield and associated mineralization. It focuses on new U-Pb (TIMS) and Sm-Nd geochronological data which constrain timing of magmatic pulses and the duration of the emplacement of $\mathrm{Cr}, \mathrm{Cu}, \mathrm{Ni}$, Ti and PGE-bearing layered intrusions of the Kola Belt. Nd, Sr and He-isotope data help define geodynamic models for a long-lived early Precambrian mantle source expressed either in a large mantle diapir or multiple plume processes for one of the earliest clearly identifiable old intraplate LIPs and its metallogeny.

\section{The Monchepluton, intrusions of the main ridge (Monchetundra and Chunatundra) and adjacent intrusions - Monchegorsk layered complex}

The Monchegorsk Layered Complex (Fig. 2) has long been the subject of detailed investigation due to the exploitation of rich $\mathrm{Cu}-\mathrm{Ni}$ ores of the Monchepluton (Papunen \& Gorbunov 1985; Chashshin et al. 2002; Smolkin et al. 2004). The complex is located at a triple junction (Fig. 2) where weakly metamorphosed early Proterozoic rift-related rocks and deep-seated Archaean rocks metamorphosed at granulite to amphibolite facies become contiguous at the modem erosion level. The Monchepluton is an S-shaped body with an area of c. $65 \mathrm{~km}^{2}$. It consists of two parts which probably represent independent magma chambers.

The northwestern and central parts of the Monchepluton (NKT: Mts Nittis, Kumuzhya and Travyanaya and Mt Sopcha) are mainly composed of non-metamorphosed ultramafic rocks, which from bottom-up are represented by a 10-100 mthick basal zone of quartz-bearing norite and gabbronorite, arzburgite (100-200 m), alternating harzburgite and orthopyroxenite (250$400 \mathrm{~m}$ ), orthopyroxenite (300-700 m) with chromitite lenses (Mt Kumuzhya) and 1-5 m-thick $\mathrm{Cu}-$ Nibearing dunite-harzburgite layers (Mt Sopcha, '330 horizon'). The total thickness of the NKT intrusion expands southwards from 200-1000 m and culminates at Mt Sopcha (1600 m).

The southeastern part of the Monchepluton (NPV: Mts Nyud, Poaz and Vurechuaivench) consists mainly of 100-600 m-thick mafic rocks: basal quartz-bearing gabbronorite and norite (up to $50 \mathrm{~m}$ ), melanocratic norite with lenses of olivinebearing harzburgite and norite, orebearing 'critical horizon' with xenoliths, olivine-free mesocratic and leucocratic norite and gabbronorite, gabbronorite, leucogabbro, anorthosite with PGE mineralization (Mt Vurechuaivench). 
Both parts of the Monchepluton (NKT and NPV chambers) have a trough-like shape with a nearhorizontal floor and flanks dipping southwestwards at an angle of $20-40^{\circ}$. The complex is underlain by the Archaean gneiss and migmatite, and overlain by the Sumi rocks of the Imandra-Varzuga rift (near Mt Vurechuaivench). The intrusive rocks of the Monchepluton are cut by veins of basic to intermediate pegmatites and diorite, and by dolerite and lamprophyre dykes.

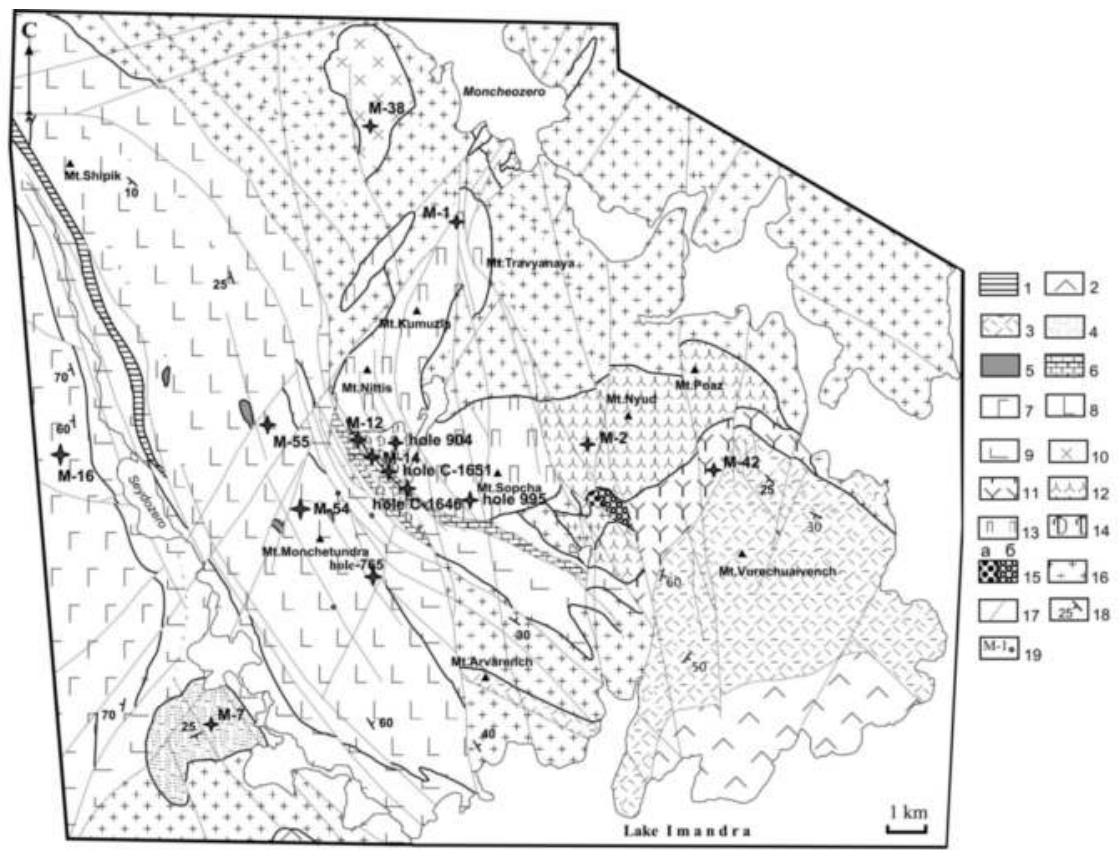

1- large gabbro-dolerite dykes, 2-metagabbroid rock of the Umbarechka-Imandra complex, 3-metasedimentary and metavolcanic rocks of the Imandra-Varzuga zone, 4 - Iherzolite, websterite, orthopyroxenite and gabbronorite of the Ostrovsky intrusion , 5 - troktolite, 6 - large norite, orthopyroxenite and gabbro dykes, 7 - gabbro-anorthosite of the Chunatundra massif, 8 - metagabbro, gabbronorite and alternating orthopyroxenite and norite of the Monchetundra intrusion, 9 - cataclasis and recrystallization of gabbroid rocks of the Chuna and Monchetundra massifs, and that of Archean amphibolite, gneiss and diorite, 10 - norite, diorite and granophyric quartz diorite of the Yarva-Varaka massif, 11-14 Monchepluton: 11 - metagabbro, gabbronorite and anorthosite of the Vurechuaivench Foothills, 12 - olivine norite, norite, gabbronorite of the Nyud-Poaz, 13 - peridotite and pyroxenite of the NKT 14 - dunite of the Sopcheozero (Dunite) Block, 15 - diorite (a) and metagabbro (6) of the Xth anomaly, 16-Archaean basement rocks; 17 - faults, 18 - dip, 19 numbered boreholes.

Figure 2. Geological map of the Monchegorsk layered complex (Smolkin et al. 2004).

The syngenetic disseminated $\mathrm{Cu}-\mathrm{Ni}$ ore occurs in layers and is usually spatially confined to the layers of olivine-bearing rocks. The ore location is controlled by the primary structural elements of the intrusions. It also may be found in the upper and basal parts of the intrusions. The mineralization is related to the coarse-grained pegmatoid rocks. Occurrences of syngenetic and nest-disseminated ore with bedded, lens-shaped and stock-like forms are locally confined 
to the parts of the intrusions where fine-grained and irregular-grained rocks, pegmatoids and rocks related to the intrusion ('critical horizon') are widely developed. The distribution of the two last-mentioned rock varieties may in some cases serve to reveal ore-controlling zones. Exploitable $\mathrm{Cu}-\mathrm{Ni}$-PGE deposits of veined epigenetic ores in the Monchepluton are confined to the systems of steeply dipping shear fractures trending NNE and dipping SSE, which trace the primary structural elements of the intrusion (geometry of intrusive blocks, primary jointing, etc.). The main ore-controlling elements in the occurrences of epigenetic stringerdisseminated ores are the zones of tectonic dislocations marked by schistose and blastomylonitized rocks. Most favourable for the concentration of injected stringer-disseminated ores are the places where the tectonic zones pass along the bend of the contact between rocks sharply different in physico-mechanical properties, for example between ultramafic rocks and Archaean granite-gneiss. The epigenetic sulphide $\mathrm{Ni}-\mathrm{Cu}$ ores of the complex tend to occur in bodies with a mainly NE strike and SW plunge.

The rocks of the Monchepluton were dated earlier by U-Pb methods on zircons and baddeleyite at the Geological Institute KSC RAS (Bayanova 2004) and at the Royal Ontario Museum laboratory in Canada (Amelin et al. 1995) with a good convergence of results (see below). These ages fall in the range of 2507-2490 Ma and favour the correlation of the Monchepluton maficultramafic layered series with the mafic layered series of the second intrusive phase of the Fedorovo-Pansky massif. In both intrusions, the main phase melts have produced $\mathrm{Cu}-\mathrm{Ni}-\mathrm{PGE}$ economic mineralization where base metals predominate, but the portion of platinum in the PGE disseminated occurrences is at least $20 \%$. The ore bodies within the ultramafic rocks of the Monchepluton (Papunen \& Gorbunov et al. 1985) are considerably richer than those of the Fedorov block deposit (Schissel et al. 2002). However, the deposits of the Monchegorsk region have already been mined out, while the Fedorovo-Pansky Complex is now being carefully investigated for future development.

Extensive areas of the Monchegorsk ore region are occupied by amphibolite-facies highpressure garnet-bearing gabbronorite-anorthosite and anorthosite with numerous conformable and cutting veins of leucogabbro and pegmatoid rocks. These are the intrusions of the Main Ridge and Lapland-Kolvitsa granulite belts (Pyrchin, etc.) located within strongly metamorphosed country rocks.

The rocks of the intrusions are insufficiently studied by modern geological and petrological methods, but have been investigated by mining companies because of the presence of high PGE and V-Ti concentrations. The Monchetundra intrusion is separated from the Monchepluton by a thick (a few hundreds of metres) blastomylonite zone with a garnet-amphibole mineral association (Smolkin et al. 2004). Regional shear zones cut and transform the primary monolith-like shape of the intrusion composed of roughly layered leucocratic mafic rocks. This results in the lens-like morphology of the intrusions.

Available U-Pb isotope ages of these anorthosites fall in a wide time interval (Mitrofanov \& Nerovich 2003; Bayanova 2004). The zircons derived from magmatic plagioclase yield an age varying from 2500-2460 Ma for different intrusions. A few generations of metamorphic zircons yield an age of multistage metamorphism that took place 2420, 1940 and 1900 Ma (Mitrofanov \& Nerovich 2003). 


\section{Monchegorsk layered complex, isotope data}

Ten samples of 50-120 kg were collected for U-Pb dating. Accessory baddeleyite and zircon were better preserved in drill core samples than in outcrops.

The oldest rocks studied are pegmatites of gabbronorite composition, which are associated with the ore-bearing sulphide veins from the basal zone of Mt Travyanaya and the 'critical horizon' (Mt Hyud, Terassa deposit). Two baddeleyite and three zircon populations were examined from these rocks. All the crystals were unaltered. Baddeleyite grains are up to 80 $\mu \mathrm{m}$ long and light brown in colour. Zircons are prismatic and isometric, up to $150 \mu \mathrm{m}$ in size, and feature narrow igneous zoning and various hues of brown. $\mathrm{U}$ and $\mathrm{Pb}$ concentrations are high, which is typical of pegmatite. A U-Pb age obtained on the five zircon and baddeleyite populations is $2500 \pm 5 \mathrm{Ma}, \mathrm{MSWD}=1.7$; the lower intersection of the discordia and the concordia is at $349 \pm 81 \mathrm{Ma}$, indicating Palaeozoic lead losses (Fig. 3A, Table 1). This age is comparable with that of $2493 \pm 7 \mathrm{Ma}$ obtained for gabbronorite of Mt Nyud, and with a zircon age for the norite of Mt Travyanaya (Fig. 3B, Table 1). A U-Pb age on baddeleyite and zircon recently obtained for the coarse-grained gabbronorite of Mt Vurechuaivench foothills (now considered as a PGE-bearing reef) is $2497 \pm 21 \mathrm{Ma}$, being very similar to that for the Fedorovo-Pansky gabbronorite (Fig. 3C, Table 1).

To determine the age of the Sopcheozero chromite deposit located within the Dunite Block of the Monchepluton, cross-cutting dykes were analysed. The Dunite Block is composed of rocks poor in accessory minerals. The dykes are assumed to be associated with intrusive mafic rocks of the Monchepluton and are thought to have intruded the Dunite Block rocks before they had cooled. Thus the age of the dykes would constrain the minimum age limit of the Dunite Block and Sopcheozero deposit formation. For U-Pb dating, a sample was collected from Borehole 1586 at a depth of 63-125 m, from a coarse-grained gabbronorite dyke cutting the ultramafic rocks of the Dunite Block. Baddeleyite, two zircon populations and rutile were used for dating. Brown transparent plate-like baddeleyite grains of up to 70-80 $\mu \mathrm{m}$ in size are well preserved. Light-ink zircons of up to $150 \mu \mathrm{m}$ in size have good outlines and thin zoning. The $\mathrm{U}-\mathrm{Pb}$ age on zircon and baddeleyite is $2496 \pm 14 \mathrm{Ma}, \mathrm{MSWD}=0.011$; the lower intersection of the discordia with the concordia is at $313 \pm 271 \mathrm{Ma}$ (Fig. 3D, Table 1). The point for the rutile has a near concordant value of c. $1.84 \mathrm{Ga}$ that reflects the time of its formation. A similar $\mathrm{U}-\mathrm{Pb}$ age $(2506 \pm 10 \mathrm{Ma})$ has also been obtained on zircon from a coarse-grained gabbronorite dyke from Borehole 1518 (Fig. 3E, Table 1). The gabbronorite dyke cuts the ultramafic rocks of the Dunite Block, therefore the Dunite Block must be older than the Monchepluton.

Small intrusions and dykes of the Monchegorsk Layered Complex were considered by most geologists to have the same age as the Monchepluton. In order to verify these relationships, diorite of the Yarva-Varaka intrusion was studied. Three zircon types and baddeleyite were selected from a sample of quartz diorite and granophyric hypersthenes diorite collected in the upper part of the Yarva-Varaka section. Stubby prismatic, pink-brown zircons of up to $150 \mu \mathrm{m}$ in size were divided by their colour hues into three populations. In i $\mu$ mersion view, they are multi-zoned. Baddeleyite grains and fragments are prismatic in habit, lightbrown coloured 
and up to $80 \mu \mathrm{m}$ in size. A $\mathrm{U}-\mathrm{Pb}$ age obtained on four points is $2496 \pm 9 \mathrm{Ma}, \mathrm{MSWD}=0.93$; the lower intersection is at zero, indicating recent lead losses (Fig. 3F, Table 1).

The Ostrovsky intrusion also belongs to the series of small mafic-ultramafic intrusions of the Monchegorsk Layered Complex. It was considered to correlate in age with the Monchepluton and was interesting as a target for $\mathrm{Cu}-\mathrm{Ni}$ prospecting. A sample for $\mathrm{U}-\mathrm{Pb}$ dating was taken from mafic pegmatite veins in the middle part of the upper gabbronorite zone (Mt Ostrovskaya). The pegmatite body is $>1 \mathrm{~m}$-thick, up to $2 \mathrm{~m}$ long and has a complex morphology, with sinuous contacts with the coarsegrained slightly amphibolized host pigeonite gabbronorite. The sample is dominated by coarsegrained to pegmatoid gabbronorites with a poikilitic texture, made up mostly of calcic plagioclase and amphibolized clinopyroxene. The $60 \mathrm{~kg}$ sample produced two types of baddeleyite and two types of zircon. Baddeleyite grains of type 1 are up to $80 \mu \mathrm{m}$ in size, with a deep-brown colour and flattened and tabular structure. Larger, up to $120 \mu \mathrm{m}$ baddeleyite grains of type 2 were found within a fringe of metamict zircon and were exposed to aeroabrasion for 15 minutes in order to remove the metamict fringe. Zircons are prismatic, up to $125 \mu \mathrm{m}$ in size, and are subdivided into light brown and brown varieties. Zircons show well-developed joints and thin zoning in i $\mu$ mersion view. The $\mathrm{U}-\mathrm{Pb}$ isochron age on two baddeleyite and two zircon points is $2445 \pm 11 \mathrm{Ma}, \mathrm{MSWD}=0.12$ and the lower intersection of the discordia with the concordia is at 500 $\pm 99 \mathrm{Ma}$ (Fig. 3G, Table 1).

To establish age correlations between the gabbronorite of the Monchepluton and the anorthosite of the Main Ridge intrusion, rock samples of the Monchetundra and Chunatundra intrusions were studied.

The Monchetundra intrusion has a complex structure and an overview of geological and geochronological investigations is given by Smolkin et al. (2004). It includes the upper zone comprised mainly of amphibolized gabbronorite and gabbro-anorthosite, and the lower zone, which consists of gabbronorite, norite and plagiopyroxenite (drilled by the deep borehole M-1).

The middle part of the upper zone, which contains a prominent horizon of slightlyaltered medium to coarse-grained gabbronorite with trachytoid texture, was sampled for $\mathrm{U}-\mathrm{Pb}$ dating. The sample yielded three zircon types. Prismatic acicular crystals up to 200 $\mu \mathrm{m}$ in size and their brown fragments were divided into three types by colour. In i $\mu$ mersion view, multi-zoning, mineral inclusions, strong jointing, corrosion of the surface and spotted uneven grain colour are observed. The $\mathrm{U}-\mathrm{Pb}$ ages (Fig. $3 \mathrm{H}$, Table 1) on zircon from trachytoid gabbronorite are 2505 $\pm 6 \mathrm{Ma}, \mathrm{MSWD}=0.31$ and $2501 \pm 8 \mathrm{Ma}$, (Fig. 3I, Table 1) $\mathrm{MSWD}=3$ (Bayanova \& Mitrofanov 2005).

A sample was also taken from the rocks of the differentiated series of the Chunatundra intrusion. Zircons from medium-grained leucogabbro with trachytoid texture were divided into five types. Four types are up to $150 \mu \mathrm{m}$ isometric fragments of brown and pink colour, whereas the last fraction is represented by up to $120 \mu \mathrm{m}$ twinned pinkishbrown zircons with adamantine lustre. The $\mathrm{U}-\mathrm{Pb}$ isochron plotted on five points has the upper intersection with the concordia at $2467 \pm 7 \mathrm{Ma}, \mathrm{MSWD}=1.4$ and the lower intersection is at zero (Fig. 3J, Table 1). This age is close to the age obtained on magmatic zircon from anorthosite of the Pyrshin 
intrusion (Mitrofanov \& Nerovich 2003) and on zircons from later anorthositic injections of the LLH (Fedorovo-Pansky Complex).
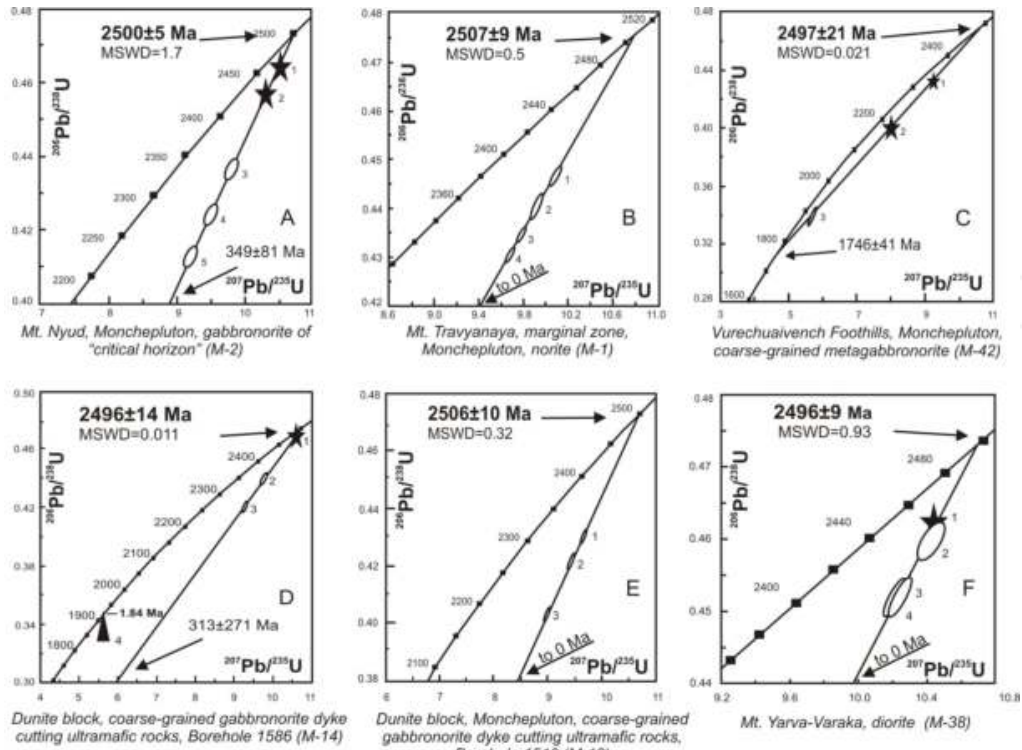
Borehole $1518(M-12)$
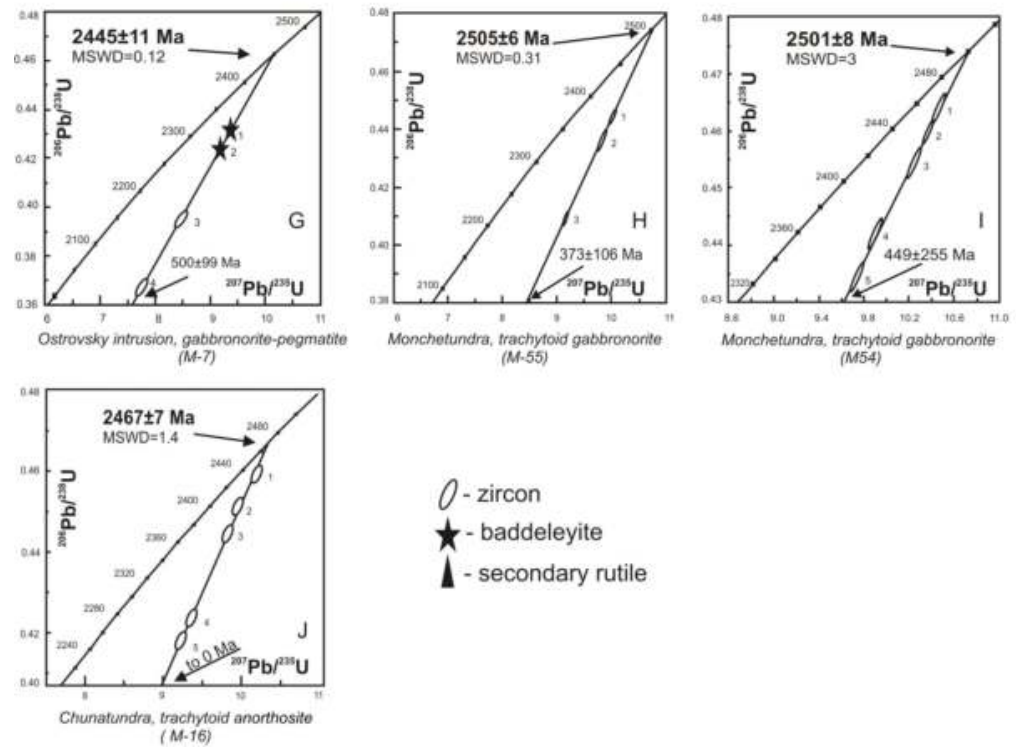

O-zircon

$\star$ - baddeleyite

\- secondary rutile

Figure 3. U-Pb concordia diagrams for zircon, baddeleyite and rutile from different rocks of the Monchegorsk Layered Complex. 


\begin{tabular}{|c|c|c|c|c|c|c|c|c|c|}
\hline \multirow{4}{*}{$\frac{\frac{0}{0}}{\frac{\sum^{2}}{N}}$} & \multirow{4}{*}{ है } & \multirow{2}{*}{\multicolumn{2}{|c|}{$\frac{\text { Concentration }}{(\mathrm{ppm})}$}} & \multirow{2}{*}{\multicolumn{3}{|c|}{$\mathrm{Pb}$ isotopic composition ${ }^{1}$}} & \multirow{2}{*}{\multicolumn{2}{|c|}{ Isotopic ratios ${ }^{2}$}} & \multirow{4}{*}{$\begin{array}{l}\mathrm{Age}^{2} \\
\text { (Ma) } \\
{ }^{207} \mathrm{~Pb} \\
{ }^{206} \mathrm{~Pb}\end{array}$} \\
\hline & & & & & & & & & \\
\hline & & \multirow{2}{*}{$\mathrm{Pb}$} & \multirow{2}{*}{ U } & ${ }^{206} \mathrm{~Pb}$ & ${ }^{206} \mathrm{~Pb}$ & ${ }^{206} \mathrm{~Pb}$ & ${ }^{207} \mathrm{~Pb}$ & ${ }^{206} \mathrm{~Pb}$ & \\
\hline & & & & ${ }^{204} \mathrm{~Pb}$ & ${ }^{207} \mathrm{~Pb}$ & ${ }^{208} \mathrm{~Pb}$ & ${ }^{235} \mathrm{U}$ & ${ }^{238} \mathrm{U}$ & \\
\hline \multicolumn{10}{|c|}{ ("critical horizon", Mt. Nyud Monchepluton, gabbronorite (M-2); from Bayanova, 2004) } \\
\hline $1(b d)$ & 0.70 & 93.1 & 198.8 & 9432 & 6.0586 & 103.8300 & 10.4643 & 0.4636 & 2495 \\
\hline $2(b d)$ & 0.40 & 170.8 & 364.4 & 3589 & 5.9833 & 50.7070 & 10.3199 & 0.4574 & 2494 \\
\hline 3 & 0.40 & 117.4 & 183.1 & 4590 & 6.0153 & 1.8703 & 9.8274 & 0.4359 & 2492 \\
\hline 4 & 0.80 & 187.9 & 308.4 & 13664 & 6.1169 & 1.9750 & 9.4740 & 0.4227 & 2483 \\
\hline \multirow[t]{2}{*}{5} & 0.50 & 152.2 & 252.5 & 5300 & 6.0842 & 1.8994 & 9.2129 & 0.4125 & 2477 \\
\hline & \multicolumn{8}{|c|}{ (marginal zone, Mt. Travyanaya Monchepluton, norite (M-1); from Smolkin et al., 2004) } & \\
\hline 1 & 0.30 & 308.3 & 504.8 & 5778 & 6.0202 & 2.3805 & 10.0760 & 0.4458 & 2497 \\
\hline 2 & 0.35 & 185.4 & 319.8 & 8358 & 6.0582 & 2.7721 & 9.9277 & 0.4402 & 2493 \\
\hline 3 & 0.40 & 264.5 & 441.6 & 23762 & 6.1006 & 2.2929 & 9.7814 & 0.4342 & 2491 \\
\hline 4 & 0.40 & 434.8 & 793.1 & 6273 & 6.0541 & 3.2613 & 9.7060 & 0.4314 & 2489 \\
\hline \multicolumn{10}{|c|}{ (Vurechuaivench Foothills Monchepluton, coarse-grained metagabbronorite (M-42); present study) } \\
\hline $1(b d)$ & 0.80 & 150.1 & 271.4 & 2982 & 6.3099 & 3.2054 & 9.23762 & 0.43446 & 2393 \\
\hline $2(b d)$ & 0.65 & 65.1 & 122.6 & 2080 & 6.5863 & 2.7920 & 8.13574 & 0.40516 & 2295 \\
\hline 3 & 0.75 & 137.4 & 288.5 & 911 & 6.4805 & 2.3018 & 5.75090 & 0.34208 & 2228 \\
\hline
\end{tabular}

(Dunite block, Monchepluton, coarse-grained gabbronorite dyke cutting ultramafic rocks, hole 1586 (M-14); from Bayanova, 2004)

\begin{tabular}{cccccccccc}
\hline $1(b d)$ & 0.50 & 5.3 & 10.3 & 1307 & 5.7748 & 12.4320 & 10.5720 & 0.4684 & 2494 \\
\hline 2 & 0.80 & 358.7 & 309.2 & 13360 & 6.1029 & 0.5312 & 9.8622 & 0.4391 & 2486 \\
\hline 3 & 0.60 & 321.8 & 362.1 & 3791 & 6.0407 & 0.7838 & 9.3919 & 0.4199 & 2479 \\
\hline $4(\text { ru })^{3}$ & 1.30 & 7.5 & 4.5 & 28 & 1.7085 & 0.8077 & 5.7139 & 0.3328 & 2022 \\
\hline
\end{tabular}

(Dunite block, Monchepluton, coarse-grained gabbronorite dyke cutting ultramafic rocks, hole 518 (M-12); from Smolkin et al., 2004)

\begin{tabular}{cccccccccc}
\hline 1 & 0.45 & 221.4 & 409.6 & 2152 & 5.9237 & 3.5776 & 9.6682 & 0.4303 & 2487 \\
\hline 2 & 0.30 & 321.7 & 542.6 & 11260 & 6.1264 & 2.2049 & 9.4976 & 0.4249 & 2478 \\
\hline 3 & 0.50 & 164.9 & 302.4 & 1952 & 5.9508 & 2.5602 & 8.9806 & 0.4031 & 2472 \\
\hline \multicolumn{7}{c}{$($ Mt. Yarva-Varaka, diorite (M-38); from Bayanova, 2004) } & & & \\
\hline $1(b d) 1$ & 0.50 & 32.9 & 70.0 & 5615 & 6.0158 & 53.309 & 10.419 & 0.4608 & 2497 \\
\hline 2 & 0.80 & 310.5 & 515.4 & 2587 & 5.9089 & 2.9118 & 10.420 & 0.4597 & 2501 \\
\hline 3 & 1.40 & 151.8 & 262.7 & 4840 & 5.9895 & 3.1873 & 10.242 & 0.4519 & 2501 \\
\hline
\end{tabular}




\begin{tabular}{|c|c|c|c|c|c|c|c|c|c|}
\hline \multirow{4}{*}{$\frac{\frac{0}{0}}{\frac{2}{n}}$} & \multirow{4}{*}{ 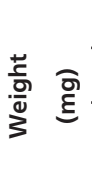 } & \multirow{2}{*}{\multicolumn{2}{|c|}{$\frac{\text { Concentration }}{(p p m)}$}} & \multirow{2}{*}{\multicolumn{3}{|c|}{$\mathrm{Pb}$ isotopic composition ${ }^{1}$}} & \multirow{2}{*}{\multicolumn{2}{|c|}{ Isotopic ratios ${ }^{2}$}} & \multirow{4}{*}{$\begin{array}{c}\mathbf{A g e}^{2} \\
\mathbf{( M a )} \\
{ }^{207} \mathrm{~Pb} \\
{ }^{206} \mathrm{~Pb}\end{array}$} \\
\hline & & & & & & & & & \\
\hline & & \multirow{2}{*}{$\mathrm{Pb}$} & \multirow{2}{*}{$U$} & ${ }^{206} \mathrm{~Pb}$ & & ${ }^{206} \mathrm{~Pb}$ & ${ }^{207} \mathrm{~Pb}$ & ${ }^{206} \mathrm{~Pb}$ & \\
\hline & & & & ${ }^{204} \mathrm{~Pb}$ & ${ }^{207} \mathrm{~Pb}$ & ${ }^{208} \mathrm{~Pb}$ & ${ }^{235} \mathrm{U}$ & ${ }^{238} \mathrm{U}$ & \\
\hline 4 & 0.70 & 273.2 & 472.5 & 4590 & 5.9970 & 3.1828 & 10.217 & 0.4518 & 2497 \\
\hline \multicolumn{10}{|c|}{ (Ostrovsky intrusion, gabbronorite-pegmatite (M-7); from Bayanova, 2004) } \\
\hline $1(b d)$ & 0.45 & 28.6 & 63.9 & 1820 & 6.162 & 29.610 & 9.350 & 0.4311 & 2405 \\
\hline $2(b d)$ & 0.55 & 36.3 & 83.7 & 3380 & 6.346 & 42.038 & 9.210 & 0.4248 & 2389 \\
\hline 3 & 0.45 & 336.4 & 694.8 & 9420 & 6.378 & 3.797 & 8.471 & 0.3953 & 2407 \\
\hline 4 & 0.35 & 89.1 & 187.6 & 3700 & 6.375 & 2.942 & 7.756 & 0.3667 & 2384 \\
\hline & & \multicolumn{7}{|c|}{ (Monchetundra, trachytoid gabbronorite (M-55); from Smolkin et al., 2004) } & \\
\hline 1 & 0.50 & 110.9 & 172.9 & 8690 & 6.0591 & 1.9737 & 10.0171 & 0.444092 & 2493 \\
\hline 2 & 0.35 & 37.7 & 61.0 & 1122 & 5.7260 & 2.2170 & 9.82794 & 0.436123 & 2492 \\
\hline 3 & 0.25 & 168.3 & 277.7 & 6350 & 6.0914 & 1.8221 & 9.15540 & 0.409417 & 2479 \\
\hline 4 & 0.30 & 122.6 & 213.5 & 6159 & 6.2294 & 1.9243 & 8.64155 & 0.395489 & 2439 \\
\hline \multicolumn{10}{|c|}{ (Monchetundra, trachytoid gabbronorite (M-54); from Smolkin et al., 2004) } \\
\hline 1 & 0.50 & 308.9 & 494.5 & 9172 & 6.0283 & 2.4881 & 10.47020 & 0.46359 & 2503 \\
\hline 2 & 0.35 & 374.3 & 587.5 & 18868 & 6.0791 & 2.2742 & 10.40220 & 0.46050 & 2496 \\
\hline 3 & 0.40 & 72.8 & 118.6 & 6833 & 6.0271 & 2.5023 & 10.25210 & 0.45405 & 2498 \\
\hline 4 & 0.25 & 206.3 & 333.1 & 7831 & 6.0324 & 2.1148 & 9.90668 & 0.44177 & 2499 \\
\hline 5 & 0.45 & 196.6 & 311.9 & 14844 & 6.1123 & 1.9269 & 9.74196 & 0.43412 & 2484 \\
\hline \multicolumn{10}{|c|}{ (Chunatundra, trachytoid anorthosite (M-16); from Bayanova, 2004) } \\
\hline 1 & 0.20 & 80.3 & 138.4 & 2710 & 6.020 & 3.404 & 10.216 & 0.4589 & 2471 \\
\hline 2 & 2.05 & 122.83 & 214.1 & 4420 & 6.144 & 3.293 & 9.999 & 0.4527 & 2455 \\
\hline 3 & 0.30 & 141.3 & 251.0 & 5140 & 6.137 & 3.291 & 9.831 & 0.4443 & 2461 \\
\hline 4 & 0.60 & 92.7 & 169.4 & 7860 & 6.148 & 2.970 & 9.388 & 0.4228 & 2467 \\
\hline 5 & 0.20 & 46.5 & 86.1 & 1090 & 5.805 & 3.242 & 9.262 & 0.4180 & 2463 \\
\hline
\end{tabular}

${ }^{1}$ All ratios are corrected for blanks of $0.08 \mathrm{ng}$ for $\mathrm{Pb}$ и $0.04 \mathrm{ng}$ for $\mathrm{U}$ and for mass discrimination of $0.12 \pm 0.04 \%$.

${ }^{2}$ Correction for common Pb was determined for the age according to Stacey and Kramers (1975).

${ }^{3}$ Corrected for isotope composition of light cogenetic plagioclase: ${ }^{206} \mathrm{~Pb} /{ }^{204} \mathrm{~Pb}=14.041 \pm 0.005,{ }^{207} \mathrm{~Pb} / 204 \mathrm{~Pb}=14.581 \pm 0.007$, ${ }^{208} \mathrm{~Pb} /{ }^{204} \mathrm{~Pb}=35.58 \pm 0.02$.

Table $1 \mathrm{U}-\mathrm{Pb}$ baddeleyite (bd), zircon and rutile (ru) isotope data from the Monchegorsk Layered Complex. 


\section{The Fedorovo-Pansky complex}

The Fedorovo-Pansky Layered Complex (Fig. 4) outcrops over an area of $>400 \mathrm{~km}^{2}$. It strikes northwestwards for $>60 \mathrm{~km}$ and dips southwestwards at an angle of 30-35. The total rock sequence is about $3-4 \mathrm{~km}$ thick. Tectonic faults divide the complex into several blocks. The major blocks from west to east (Fig. 4) are known as the Fedorov, the Lastjavr, the Western Pansky and the Eastern Pansky (Mitrofanov et al. 2005). The Fedorovo-Pansky complex is bordered by the Archaean Keivy terrane and the Palaeoproterozoic Imandra-Varzuga rift. The rocks of the complex crop out close to the Archaean gneisses only in the northwestern extremities, but their contacts cannot be established due to poor exposure. In the north, the complex borders with the alkaline granites of the White Tundra intrusion. The alkaline granites were recently proved to be Archaean with a U-Pb zircon age of $2654 \pm 15 \mathrm{Ma}$ (Bayanova 2004; Zozulya et al. 2005). The contact of the Western Pansky Block with the Imandra-Varzuga volcanosedimentary sequence is mostly covered by Quaternary deposits. However, drilling and excavations in the south of Mt Kamennik reveal a strongly sheared and metamorphosed contact between the intrusion and overlying Palaeoproterozoic volcano-sedimentary rocks that we interpret to be tectonic in origin.

The Fedorovo-Pansky Complex comprises predominantly gabbronorites with varying proportions of mafic minerals and different structural features. From bottom up, the composite layered sequence is as follows:

- Marginal Zone (50-100 m) of plagioclase-amphibole schists with relicts of massive finegrained norite and gabbronorite, which are referred to as chilled margin rocks;

- Taxitic Zone (30-300 m) that contains orebearing gabbronoritic matrix (2485 Ma, see below) and early xenoliths of plagioclasebearing pyroxenite and norite (2526-2516 Ma, see below). Syngenetic and magmatic ores are represented by $\mathrm{Cu}$ and $\mathrm{Ni}$ sulphides with $\mathrm{Pt}, \mathrm{Pd}$ and $\mathrm{Au}$, and $\mathrm{Pt}$ and Pd sulphides, bismuthotellurides and arsenides;

- Norite Zone (50-200 m) with cumulus interlayers of harzburgite and plagioclase-bearing pyroxenite that includes an intergranular injection $\mathrm{Cu}-\mathrm{Ni}-\mathrm{PGE}$ mineralization in the lower part. The rocks of the zone are enriched in chromium (up to $1000 \mathrm{ppm}$ ) and contain chromite that is also typical of the rocks of the Penikat and Kemi intrusions (Finland) derived from the earliest magma portion (Iljina \& Hanski 2005). Basal Cu-Ni-PGE deposits of the Fedorov Block have been explored and prepared for licensing (Schissel et al. 2002; Mitrofanov et al. 2005).

- Main Gabbronorite Zone (c. $1000 \mathrm{~m}$ ) that is a thickly layered 'stratified' rock series (Fig. 4) with a 40-80 m thinly layered lower horizon (LLH) at the upper part. The LLH consists of contrasting alteration of gabbronorite, norite, pyroxenite and interlayers of leucocratic gabbro and anorthosite. The LLH contains a reef-type PGE deposit poor in base-metal sulphides. The deposit is now being extensively explored (Mitrofanov et al. 2005). According to the field investigations (Latypov \& Chistyakova 2000), the LLH anorthositic layers have been intruded later, as shown by cutting injection contacts. This is confirmed by a zircon U$\mathrm{Pb}$ age for the anorthosite of $2470 \pm 9 \mathrm{Ma}$ (see below). 
- Upper Layered Horizon (ULH) between the Lower and Upper Gabbro Zones. The ULH consists of olivine-bearing troctolite, norite, gabbronorite and anorthosite (Fig. 4). It comprises several layers of rich PGE (Pd-Pt) ore poor in base-metal sulphides (Mitrofanov et al. 2005). The $\mathrm{U}-\mathrm{Pb}$ age on zircon and baddeleyite of the ULH rocks of $2447 \pm 12$ Ma (see below) is the youngest among those obtained for the rocks of the FedorovoPansky Complex.

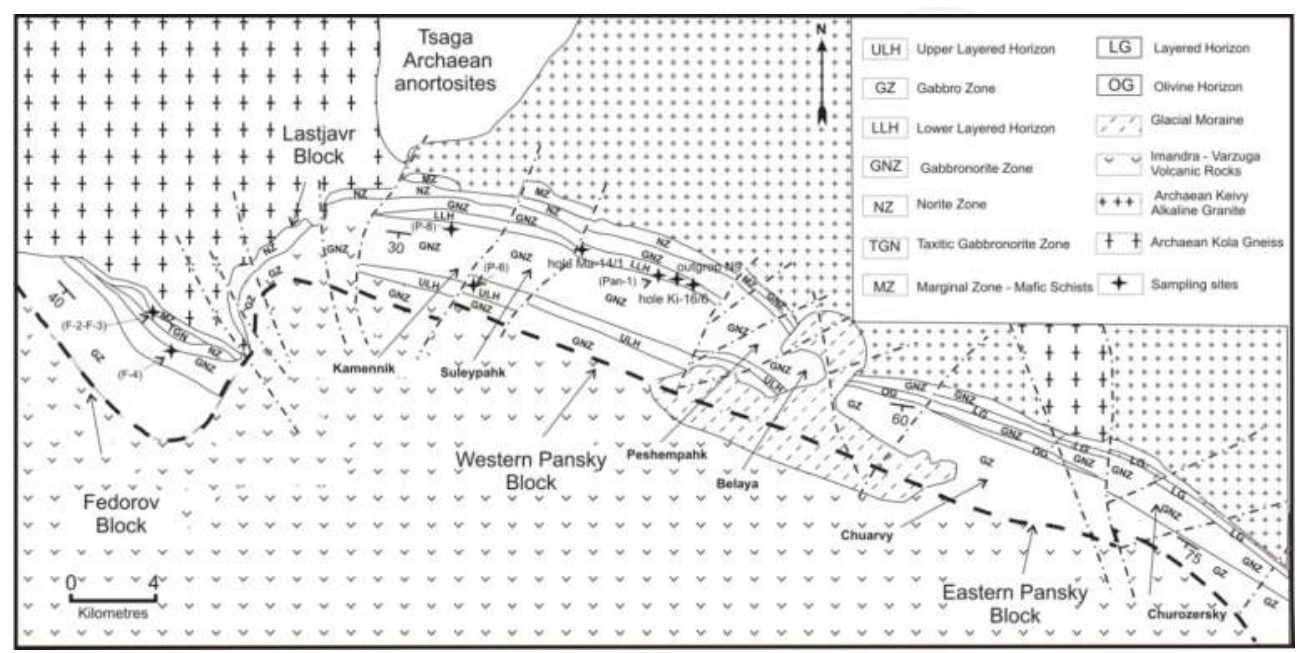

Figure 4. General geological map of the Fedorovo-Pansky Layered Complex (Mitrofanov et al. 2005).

\section{Fedorovo-Pansky complex, isotope data}

Several large samples were selected for the U-Pb dating of the Fedorovo-Pansky Complex.

A $60 \mathrm{~kg}$ sample of medium-and coarse-grained gabbronorite was collected from the Lower Layered Horizon in the Eastern Kievey area. The separated zircons are transparent with a vitreous lustre. All the grains were divided into three types: Pan-1-regular bipyramidalprismatic crystals of up to $120 \mu \mathrm{m}$; Pan-2-fragments of prismatic crystals; Pan-3-pyramidal apices of crystals of $80-100 \mu \mathrm{m}$. In i with fine zoning and cross jointing.

The discordia plotted on three points yields the upper intersection with the concordia and the $\mathrm{U}-\mathrm{Pb}$ age at $2491 \pm 1.5 \mathrm{Ma}, \mathrm{MSWD}=0.05$. The lower intersection of the discordia with the Concordia is at zero and reflects modern lead losses (Fig. 5A, Table 2). The same zircon sample was analysed in the Royal Ontario Museum laboratory in Canada; the obtained $\mathrm{U}-\mathrm{Pb}$ zircon 
age is $2501.5 \pm 1.7 \mathrm{Ma}$ (Amelin et al. 1995) that is somewhat older than ours. The age obtained is interpreted as the time of crystallization of the main gabbronorite phase rock (Mitrofanov et al. 1997; Mitrofanov \& Bayanova 1999).

$\mathrm{Sm}-\mathrm{Nd}$ dating on ortho-and clinopyroxene, plagioclase and whole-rock minerals extracted from the same gabbronorite gave an age of 2487 51 Ma, MSWD=1.5 (Balashov et al. 1993).

Three zircon populations of prismatic habit and light-yellow colour were separated from PGEbearing gabbro-pegmatite (LLH). The zircons from sample P-8 are stubby prismatic crystals with sharp outlines, about $100 \mu \mathrm{m}$ in size. The crystals show cross-cracks and apparent zoning in i $\mu$ mersion view. The zircons from samples D-15 and D-18 are multi-zoned pinkish fragments of prismatic crystals with adamantine lustre and 80 and $100 \mu \mathrm{m}$ in size. The U-Pb zircon age of $2470 \pm 9 \mathrm{Ma}, \mathrm{MSWD}=0.37$ (Fig. 5A, Table 2) was obtained from three points: one concordant and two lying in the upper part of the isochron. The lower intersection of the discordia with the concordia (c. $300 \mathrm{Ma}$ ) indicates lead loss associated with the Palaeozoic tectonic activation of the eastern Baltic Shield and the development of the giant Khibina and Lovozero intrusions of nepheline syenites (Kramm et al. 1993). Zircons from the gabbro-pegmatite are found to have higher $\mathrm{U}$ and $\mathrm{Pb}$ concentrations than those from the gabbronorite.

Three zircon and two baddeleyite populations were separated from a sample collected from the Upper Layered Horizon in the Southern Suleypahk area. All the zircons from anorthosite are prismatic, light-pink-coloured with vitreous lustre. In i $\mu$ mersion view, they are zoned and fractured. A population of bipyramidal-prismatic zircons (Pb-1) is made up of elongate (3:1) crystals. Sample $\mathrm{Pb}-2$ contains zircons of round-ellipsoidal habit and sample $\mathrm{Pb}-3$ contains transparent flattened crystal fragments of up to $0.75 \mu \mathrm{m}$ in size.

The separated baddeleyite crystals (first recorded in the anorthosite) were subdivided into two varieties, deep-brown and brown. All the grains are fragments of transparent baddeleyite crystals of $50 \mu \mathrm{m}$ in size, without selvages and inclusions.

A U-Pb isochron plotted from three zircons and two baddeleyites intersects the concordia with an age of $2447 \pm 12 \mathrm{Ma}, \mathrm{MSWD}=2.7$ (Fig. 5B, Table 2). The lower intersection of the Discordia with the concordia records recent lead loss. The position of the baddeleyite points is nearconcordant, while zircon points (Sample P6-1) are above the concordia due to uranium loss. This age $(2447 \pm 12 \mathrm{Ma})$ is considered to constrain the origin of latephase anorthosite because, as shown by Heaman \& LeCheminant (1993), baddeleyite is coumonly generated in residual melts.

The U-Pb zircon age of the early barren orthopyroxenite from the Fedorov Block, $2526 \pm 6 \mathrm{Ma}$, is believed to be the time of emplacement (Fig. 5C, Table 2). The U-Pb age of 2516 $\pm 7 \mathrm{Ma}$ (Fig. $5 \mathrm{D}$, Table 2), obtained from zircon from barren olivine gabbro, is interpreted as the time of crystallization. The last $\mathrm{Cu}-\mathrm{Ni}-\mathrm{PGE}-$ bearing taxitic gabbronorite from the Fedorov Block (Fig. $5 \mathrm{E}$, Table 2) yielded a U-Pb zircon age of $2485 \pm 9 \mathrm{Ma}$ (Nitkina 2006).

Ihe coeval $\mathrm{Sm}-\mathrm{Nd}$ isotope ages have been oblained using rock-forming minerals from the same rork of the Fedorovo-Pansky massif (Fig. 6, Table 3). 

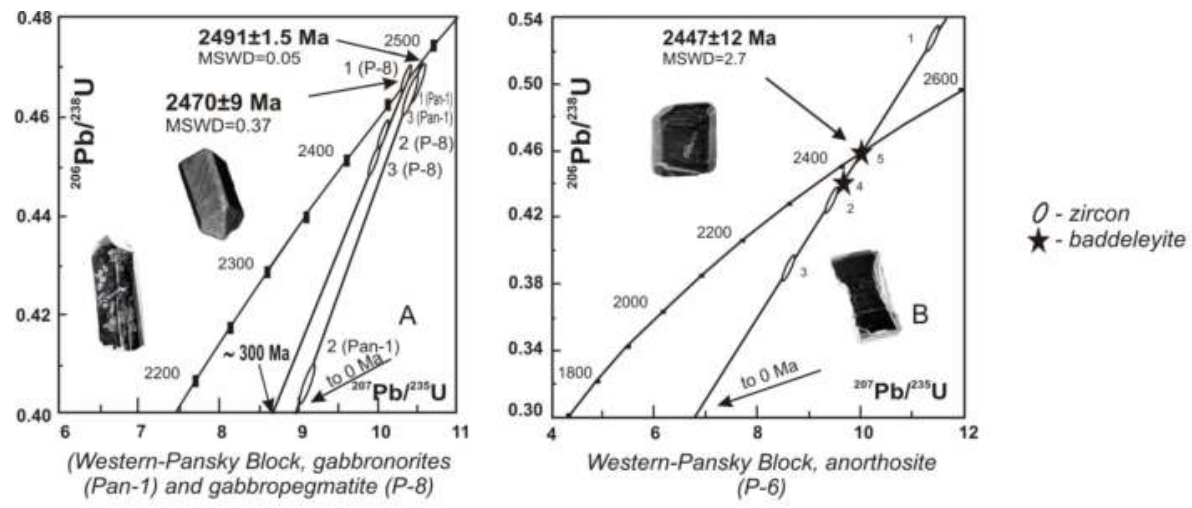

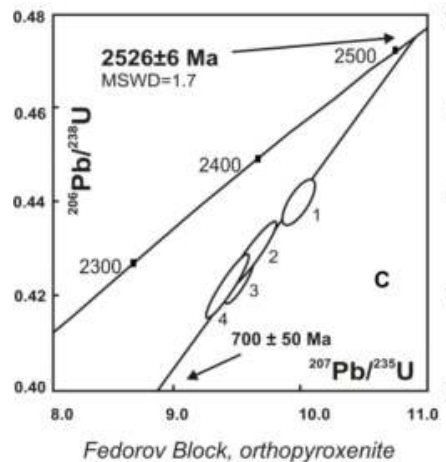

( $F-3)$

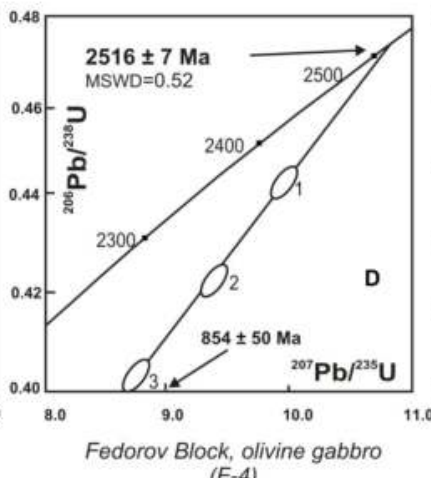

$(\mathrm{F}-4)$

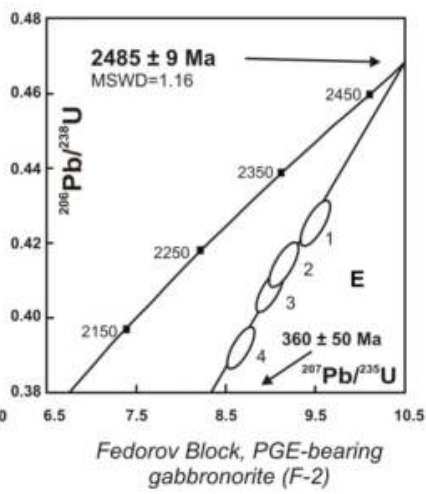

gabbronorite ( $F-2)$

Figure 5. U-Pb concordia diagrams for the Western-Pansky $(a, b)$ and Fedorov $(c, d, e)$ Blocks of the Fedorovo-Pansky Complex.

\begin{tabular}{|c|c|c|c|c|c|c|c|c|}
\hline \multirow[t]{2}{*}{ SampleNo } & \multirow{2}{*}{$\frac{\text { Weight }}{\text { (mg) }}$} & Concentration & \multicolumn{3}{|c|}{$\mathrm{Pb}$ isotopic composition ${ }^{1}$} & \multicolumn{2}{|c|}{ Isotopic ratios ${ }^{2}$} & \multirow{2}{*}{$\frac{\mathrm{Age}^{2}}{(\mathrm{Ma})}$} \\
\hline & & (ppm) & & & & & & \\
\hline & & $\mathrm{Pb}$ & ${ }^{206} \mathrm{~Pb}$ & ${ }^{206} \mathrm{~Pb}$ & ${ }^{206} \mathrm{~Pb}$ & ${ }^{207} \mathrm{~Pb}$ & ${ }^{206} \mathrm{~Pb}$ & ${ }^{207} \mathrm{~Pb}$ \\
\hline
\end{tabular}

(Western-Pansky Block, gabbronorites (Pan-1); from Bayanova, 2004)

\begin{tabular}{rrrrrrrrrr}
\hline 1 & 3.30 & 95.0 & 144 & 11740 & 6.091 & 3.551 & 10.510 & 0.4666 & 2491 \\
\hline 2 & 1.90 & 70.0 & 142 & 10300 & 6.100 & 4.220 & 9.135 & 0.4061 & 2489 \\
\hline 3 & 1.60 & 84.0 & 144 & 6720 & 6.062 & 3.552 & 10.473 & 0.4650 & 2491 \\
\hline
\end{tabular}




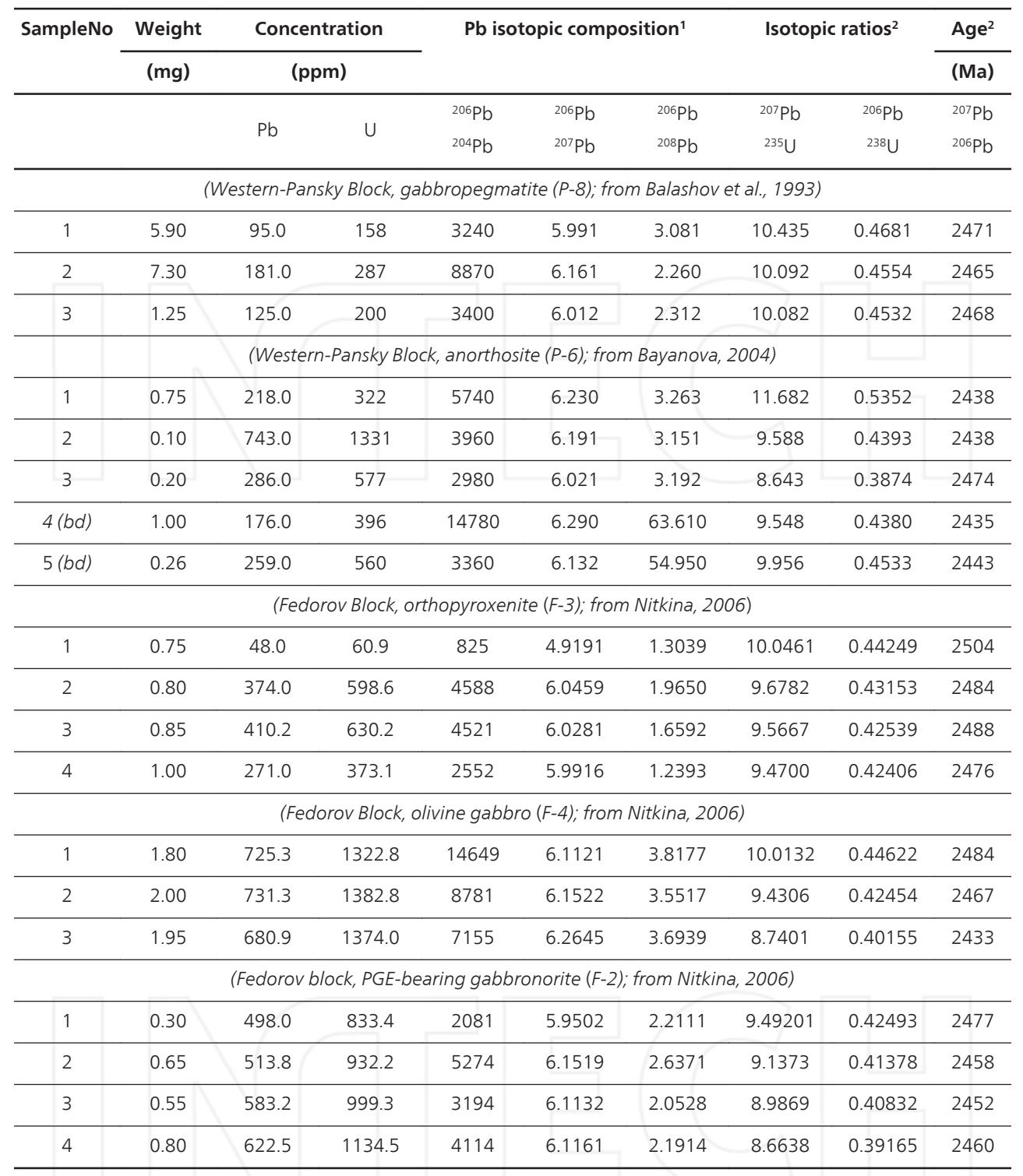

${ }^{1}$ All ratios are corrected for blanks of $0.1 \mathrm{ng}$ for $\mathrm{Pb}$ and $0.04 \mathrm{ng}$ for $\mathrm{U}$ and for mass discrimination of $0.17 \pm 0.05 \%$.

${ }^{2}$ Correction for common $\mathrm{Pb}$ was determined for the age according to Stacey and Kramers (1975).

Table $2 \mathrm{U}$-Pb baddeleyite $(\mathrm{bd})$ and zircon isotope data from the Western-Pansky and Fedorov Blocks of the FedorovoPansky Complex. 

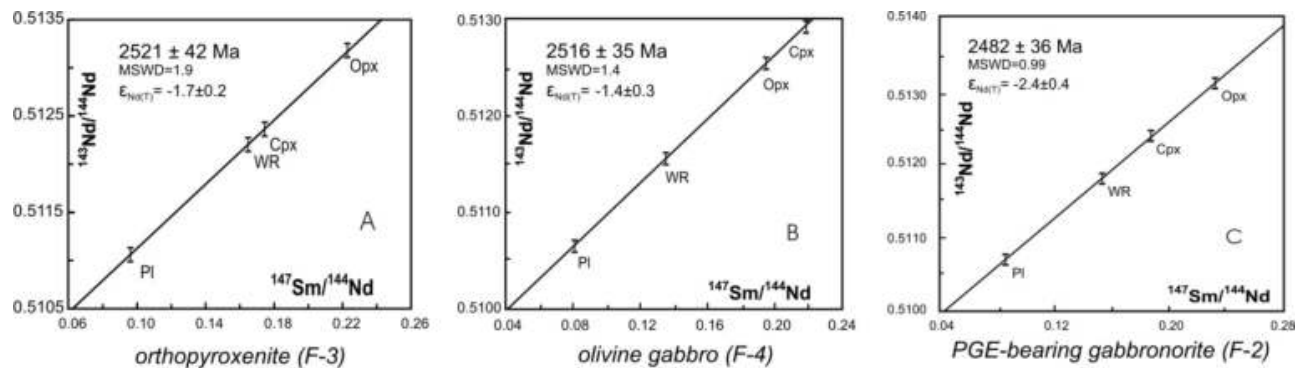

Figure 6. Mineral Sm-Nd isochrons for rocks and rock-forming minerals of the Fedorov Block of the Fedorovo-Pansky Complex.

\begin{tabular}{|c|c|c|c|c|c|c|c|}
\hline \multirow[t]{3}{*}{ Sample No } & \multirow{2}{*}{\multicolumn{2}{|c|}{$\begin{array}{c}\text { Concentration } \\
\text { (ppm) }\end{array}$}} & \multirow{2}{*}{\multicolumn{2}{|c|}{ Isotopic ratios }} & \multirow{3}{*}{$\frac{T_{D M}}{(G a)}$} & \multirow{3}{*}{$\frac{\mathrm{Sm}-\mathrm{Nd}}{(\mathrm{Ma})}$} & \multirow[t]{3}{*}{$\varepsilon_{\mathrm{Nd}(2.5 \mathrm{Ga})}$} \\
\hline & & & & & & & \\
\hline & Sm & Nd & ${ }^{147} \mathrm{Sm} /{ }^{144} \mathrm{Nd}$ & ${ }^{143} \mathrm{Nd} /{ }^{144} \mathrm{Nd}$ & & & \\
\hline \multicolumn{8}{|c|}{ orthopyroxenite (F-3) } \\
\hline WR & 0.32 & 1.17 & 0.1648 & $0.512196 \pm 12$ & 3.05 & $2521 \pm 42$ & -1.73 \\
\hline Opx & 0.12 & 0.38 & 0.2228 & $0.513182 \pm 16$ & & & \\
\hline Cpx & 2.21 & 7.67 & 0.1745 & $0.512349 \pm 17$ & & & \\
\hline $\mathrm{Pl}$ & 0.26 & 1.62 & 0.0960 & $0.511071 \pm 29$ & & & \\
\hline \multicolumn{8}{|c|}{ olivine gabbro (F-4) } \\
\hline WR & 0.63 & 2.80 & 0.1357 & 0.5115488 & 2.94 & $2516 \pm 35$ & -1.53 \\
\hline Opx & 0.23 & 0.72 & 0.1951 & 0.51255515 & & & \\
\hline Cpx & 0.83 & 2.28 & 0.2187 & 0.51294716 & & & \\
\hline $\mathrm{Pl}$ & 0.24 & 1.77 & 0.0815 & 0.51067714 & & & \\
\hline & & & \multicolumn{3}{|c|}{ PGE-bearing gabbronorite (F-2) } & & \\
\hline WR & 0.42 & 1.66 & 0.1537 & 0.51180720 & 3.18 & $2482 \pm 36$ & -2.50 \\
\hline $\mathrm{Pl}$ & 0.41 & 2.88 & 0.0865 & 0.51070914 & & & \\
\hline Cpx & 1.78 & 5.73 & 0.1876 & 0.5123878 & & & \\
\hline Opx & 0.13 & 0.33 & 0.2323 & 0.51308840 & & & \\
\hline
\end{tabular}

Table $3 \mathrm{Sm}-\mathrm{Nd}$ isotope data on whole rock and mineral separates of the Fedorov Block of the Fedorovo-Pansky Complex. 


\section{Analytical U-Pb, Sm-Nd, Rb-Sr methods}

$\mathrm{U}-\mathrm{Pb}$ (TIMS) method with ${ }^{208} \mathrm{~Pb} /{ }^{235} \mathrm{U}$ tracer. Following the method proposed by Krogh (1973), the samples were dissolved in strong (48\%) hydrofluoric acid at a temperature of $205-210{ }^{\circ} \mathrm{C}$ over 1-10 days. In order to dissolve fluorides, the samples were reacted with $3.1 \mathrm{~N} \mathrm{HCl}$ at a temperature of $130{ }^{\circ} \mathrm{C}$ for $8-10$ hours. To determine the isotope composition of lead and concentrations of lead and uranium, the sample was divided into two aliquots in $3.1 \mathrm{~N} \mathrm{HCl}$, and a mixed ${ }^{208} \mathrm{~Pb} /{ }^{235} \mathrm{U}$ tracer was added. $\mathrm{Pb}$ and $\mathrm{U}$ were separated on an $\mathrm{AG} 1 \times 8,200-400$ mesh anion exchanger in Teflon columns. The laboratory blank for the whole analysis was $<0.1-0.08 \mathrm{ng}$ for $\mathrm{Pb}$ and $0.01-0.04 \mathrm{ng}$ for $\mathrm{U}$. All isotopic determinations for zircon and baddeleyite were made on Finnigan MAT-262 and MI 1201-T mass spectrometers and the Pb isotopic composition was analysed on a secondary-ion multiplier on a Finnigan MAT-262 in ion counting mode. The measurements of the $\mathrm{Pb}$ isotopic composition are accurate to $0.025 \%$ (Finnigan MAT-262) and 0.15\% (MI 1201-T) when calibrated against NBS SRM-981 and SRM-982 standards, respectively. The $\mathrm{U}$ and $\mathrm{Pb}$ concentrations were measured in singlefilament mode with the addition of $\mathrm{H}_{3} \mathrm{PO}_{4}$ and silica gel using the method (Scharer \& Gower 1988; Scharer et al. 1996). $\mathrm{Pb}$ and $\mathrm{U}$ concentrations were measured within the temperature ranges of $1350-1450$ and $1450-1550{ }^{\circ} \mathrm{C}$, respectively. All of the isotopic ratios were corrected for mass discrimination during the static processing of replicate analyses of the SRM-981 and SRM-982 standards $(0.12 \pm 0.04 \%$ for the Finnigan MAT-262 and $0.17 \pm 0.05 \%$ per a.m.u.). The errors in the $\mathrm{U}-\mathrm{Pb}$ ratios were calculated during the statistical treatment of replicate analyses of the IGFM-87 standard and were assumed equal to $0.5 \%$ for Finnigan MAT-262 and $0.7 \%$ for MI 1201-T. If the actual analytical errors were higher, they are reported in the table of isotopic data. Isochrons and sample points were calculated Squid and Isoplot programs (Ludwig 1991, 1999). The age values were calculated with the conventional decay constants for $U$ (Steiger \& Jager 1977), all errors are reported for a 2 sigma level. Corrections for coumon $\mathrm{Pb}$ were made according to Stacey \& Kramers (1975). Corrections were also made for the composition of $\mathrm{Pb}$ separated from syngenetic plagioclase or microcline if the admixture of co $\mu$ mon $\mathrm{Pb}$ was $>10 \%$ of the overall $\mathrm{Pb}$ concentration and the ${ }^{206} \mathrm{~Pb} /{ }^{204} \mathrm{~Pb}$ ratios were $<1000$.

${ }^{205} \mathrm{~Pb} /{ }^{235} \mathrm{U}$ tracer for single grains. U-Pb (TIMS) method with based U-Pb method for single grain accessory minerals using ion-exchange chromatography. Handpicked crystals are first treated in ultrasonic bath for cleaning in spirit or in acetone, and then in $7 \mathrm{~N}$ nitric acid, heated for about 15 minutes on a warm rangette, and finally are three times flushed with recurrent purification water. Chemical mineral decomposition is performed in teflon bombs with adding 3 to $5 \mathrm{mcl}$ of mixed ${ }^{205} \mathrm{~Pb} /{ }^{235} \mathrm{U}$ tracer using T. Krogh method (1973) in concentrated nitric acid during 5 to 7 days at a temperature of $210^{\circ} \mathrm{C}$. After complete decomposition, the column effluent is evaporated on a warm ragette, and then 10 drops of $6.2 \mathrm{~N}$ chlorohydric acid are added. The sample is placed to the thermostat for 8 to 10 hours at a temperature of $140-150^{\circ} \mathrm{C}$ for homogenization. Lead and uranium are separated for isotope investigations using ion-exchange chromatography in columns with Dowex IX8 200-400 mesh resin. Lead is eluted with 10 drops of $6.2 \mathrm{~N}$ chlorohydric acid when also one drop of $0.1 \mathrm{~N}$ phosphoric acid is added, and the solution is evaporated on a ragette down to $3 \mathrm{mcl}$. Uranium is eluted separately from lead with 20 drops of water with one drop of $0.1 \mathrm{~N}$ phosphoric acid added, and evaporated 
on a ragetter down to $3 \mathrm{mcl}$. All chemical procedures are carried out in the ultraclean block with blank $\mathrm{Pb}$ and $\mathrm{U}$ contamination of ca. 1-3 pg, and ca. 10-15 pg respectively. The measurement of $\mathrm{Pb}$ and $\mathrm{U}$ isotope composition and concentrations is performed on Re bands at sevenchannel mass-spectrometer Finnigan-MAT 262 (RPG), on collectors, with ${ }^{204} \mathrm{~Pb}$ and ${ }^{205} \mathrm{~Pb}$ measured at a temperature of $1350-1450^{\circ} \mathrm{C}$ in an ion counting mode using a multiplier or quadrupole RPG accessory. Silicagel is used as an emitter. $\mathrm{U}$ concentrations are detected at a temperature of $1450-1550^{\circ} \mathrm{C}$ using a collector and a multiplier in a mixed statically dynamic mode. When $U$ concentrations are negligible, the multiplier or quadrupole RPQ accessory is applied in a dynamic mode. All the measured isotope ratios are adjusted for mass-discrimination obtained when studying parallel analyses of SRM-981 and SRM-982 standards to be $0.12 \pm 0.04 \%$. The coordinates of points and isochrone parameters are calculated using programs by K. Ludwig $(1991,1999)$. Ages are calculated in accordance with the accepted values of uranium decay constants (Steiger and Jger, 1977), with errors being indicated on a 2b level. The J. Stacey \& J. Kramers model (1975) is used to adjust numbers for the admixture of coumon lead.

Isotope $\mathrm{Sm} / \mathrm{Nd}$ method. In order to define concentrations of samarium and neodymium, the sample was mixed with a compound tracer ${ }^{149} \mathrm{Sm} /{ }^{150} \mathrm{Nd}$ prior to dissolution. It was then diluted with a mixture of $\mathrm{HF}+\mathrm{HNO}_{3}\left(\right.$ or $\left.+\mathrm{HClO}_{4}\right)$ in Teflon sample bottles at a temperature of $100{ }^{\circ} \mathrm{C}$ until complete dissolution. Further extraction of $\mathrm{Sm}$ and $\mathrm{Nd}$ was carried out using standard procedures with twostage ion-exchange and extraction-chromatographic separation using ion-exchange tar «Dowex» $50 \times 8$ in chromatographic columns employing $2.3 \mathrm{~N}$ and $4.5 \mathrm{~N} \mathrm{HCl}$ as an eluent. The separated $\mathrm{Sm}$ and $\mathrm{Nd}$ fractions were transferred into nitrate form, whereupon the samples (preparations) were ready for mass-spectrometric analysis. Measurements of Ndisotope composition and $\mathrm{Sm}$ and $\mathrm{Nd}$ concentrations by isotope dilution were performed using a multicollector mass-spectrometer in a Finnigan MAT 262 (RPQ) in a static mode using Re + Re and Ta+Re filament. The measured reproducibility for ten parallel analysis of Nd-isotope composition for the standard La Jolla $=0.511833 \pm 6$ was $<0.0024 \%(2 \sigma)$. The same reproducibility was obtained from 11 parallel analyses of the Japanese standard: Ji Nd1 $=0.512078 \pm 5$. The error in ${ }^{147} \mathrm{Sm} /{ }^{144} \mathrm{Nd}$ ratios of $0.2 \%(2 \sigma)$, the average of seven measures, was accepted for statistic calculations of $\mathrm{Sm}$ and $\mathrm{Nd}$ concentrations using the BCR standard. The blanks for laboratory contamination for $\mathrm{Nd}$ and $\mathrm{Sm}$ are 0.3 and $0.06 \mathrm{ng}$, respectively. Isochron parameters were developed from programs of Ludwig $(1991,1999)$. The reproducibility of measurements was $\pm 0.2 \%(2 \sigma)$ for $\mathrm{Sm} / \mathrm{Nd}$ ratios and $\pm 0.003 \%(2 \sigma)$ for $\mathrm{Nd}$-isotope analyses. All ${ }^{147} \mathrm{Sm} /{ }^{144} \mathrm{Nd}$ and ${ }^{143} \mathrm{Nd} /{ }^{144} \mathrm{Nd}$ ratios were normalized to ${ }^{146} \mathrm{Nd} /{ }^{144} \mathrm{Nd}=0.7219$ and adjusted to ${ }^{143} \mathrm{Nd} /{ }^{144} \mathrm{Nd} .0 .511860$ using the La Jolla Nd standard. The $\varepsilon_{\mathrm{Nd}}(\mathrm{T})$ values and model TDM ages were calculated using the currently accepted parameters of CHUR (Jacobsen \& Wasserburg 1984): ${ }^{143} \mathrm{Nd} /$ ${ }^{144} \mathrm{Nd}=0.512638$ and ${ }^{147} \mathrm{Sm} /{ }^{144} \mathrm{Nd}=0.1967$ and $\mathrm{DM}$ (Goldstein \& Jacobsen 1988): ${ }^{143} \mathrm{Nd} /$ ${ }^{144} \mathrm{Nd}=0.513151$ and ${ }^{147} \mathrm{Sm} /{ }^{144} \mathrm{Nd}=0.2136$.

Sm-Nd method for studying suphides. The chemical and analytical treatment of sulphide minerals (pyrite, pentlandite, chalcopyrite, etc.) for Sm-Nd study is performed following a modified technique (Yekimova 2011) as compared to the conventional one (Zhuravlyov et al. 1987). To decompose sulphides, a mineral weight $(20$ to $50 \mathrm{mg})$ is mixed with a ${ }^{149} \mathrm{Sm} /{ }^{150} \mathrm{Nd}$ 
tracer solution, treated with aqua regia $\left(\mathrm{HCl}+\mathrm{HNO}_{3}\right)$ until complete decomposition, and evaporated dry. Afterwards, this is transformed to chlorides through evaporating the sample in $4.5-6 \mathrm{~N} \mathrm{HCl}$. After the fractional acid decomposition, the dry residue is dissolved in $\sim 1 \mathrm{ml}$ $2.3 \mathrm{~N} \mathrm{HCl}$, and total REEs are separated from the solution via cation-exchange chromatography. A stepwise elution method is applied to 2.3 and $4.5 \mathrm{~N} \mathrm{HCl}$ in a chromatographic column with cation-exchange resin Dowex 50x8 (200-400 mesh). The separated REE fraction is evaporated dry, dissolved in $0.1 \mathrm{~N} \mathrm{HCl}$, and loaded to the second column with KEL-F solid ion-exchange resin HDEHP. The resin is used to separate Sm and $\mathrm{Nd}$. The selected Sm and $\mathrm{Nd}$ fractions are evaporated to get prepared for further mass-spectrometric analysis. All the measurements of the $\mathrm{Nd}$ isotope composition and $\mathrm{Sm}$ and $\mathrm{Nd}$ concentrations using an isotope dilution technique were performed at a seven-channel solid-phase mass-spectrometer Finnigan-MAT 262 (RPQ) in a static double-band mode in collectors using Ta+Re filaments. Re filaments were used as ionizers, and the sample was applied to the Ta filament with a diluted the $\mathrm{H}_{3} \mathrm{PO}_{4}$ microdrop being deposited on beforehand. The reproducibility error for eleven $\mathrm{Nd}$ isotope composition determinations of La Jolla $=0.511833 \pm 6(2 \sigma, \mathrm{N}=11)$ has been within $0.0024 \%(2 \sigma)$. The same error was obtained when measuring forty-four parallel analyses of a new Japanese standard, $\mathrm{JNd}_{\mathrm{i}} 1=0.512072 \pm 2(2 \sigma, \mathrm{N}=44)$. The error in ${ }^{147} \mathrm{Sm} /{ }^{144} \mathrm{Nd}$ ratios is accepted for the static calculation of the Sm and Nd concentrations in BCR-1 to be $0.2 \%(2 \sigma)$, which is an average of seven measurements. The blank intralaboratory contamination in $\mathrm{Nd}$ and in Sm is $0.3 \mathrm{ng}$ and $0.06 \mathrm{ng}$ respectively. The measured $\mathrm{Nd}$ isotope rations were normalized per ${ }^{148} \mathrm{Nd} /{ }^{144} \mathrm{Nd}=0.241570$, and recalculated for ${ }^{143} \mathrm{Nd} /{ }^{144} \mathrm{Nd}$ in LaJolla $=0.511860$ afterwards. The isochron parameters were computed using K. Ludwig programs (Ludwig 1991, 1999). The decompositions constants are as per (Steiger 1977). The $\varepsilon_{\mathrm{Nd}}$ parameters for a one-stage model were calculated using [De Paolo 1981], and for a two-stage model using (Liew \& Hofmann 1988).

Isotope $\mathrm{Rb} / \mathrm{Sr}$ method. The samples and minerals were all treated with double distilled acids ( $\mathrm{HCl}, \mathrm{HF}$ and $\mathrm{HNO}_{3}$ ) and $\mathrm{H}_{2} \mathrm{O}$ distillate. A sample of 20-100 mg (depending on $\mathrm{Rb}$ and $\mathrm{Sr}$ contents) was dissolved with $4 \mathrm{ml}$ of mixed $\mathrm{HF}$ and $\mathrm{HNO}_{3}(5: 1)$ in corked teflon sample bottles and left at a temperature of about $200{ }^{\circ} \mathrm{C}$ for one day. The solution was then divided into three aliquots in order to determine $\mathrm{Rb}$ and $\mathrm{Sr}$ isotope compositions and concentrations. These were measured by isotope dilution using separate ${ }^{85} \mathrm{Rb}$ and ${ }^{84} \mathrm{Sr}$ tracers. $\mathrm{Rb}$ and $\mathrm{Sr}$ extraction was performed by eluent chromatography with «Dowex» $\operatorname{tar} 50 \times 8$ (200-400 mesh); $1.5 \mathrm{~N}$ and 2.3 $\mathrm{N} \mathrm{HCl}$ served as an eluent. Tar volumes in the columns were c. 7 and c. $4 \mathrm{sm}^{3}$. The separated $\mathrm{Rb}$ and $\mathrm{Sr}$ fractions were evaporated until dryness, followed by treatment with a few drops of $\mathrm{HNO}^{3}$. Sr isotope compositions and $\mathrm{Rb}$ and $\mathrm{Sr}$ contents were measured by a MI-1201-T (Ukraine) mass spectrometer in the two-ribbon mode using Re filaments. The prepared samples were deposited on the ribbons in the form of nitrate. Sr isotope composition in all the measured samples was normalized to a value of 0.710235 recoumended by NBS SRM- 987 . Errors on Sr isotope analysis (confidence interval of 95\%) do not exceed $0.04 \%$, and those of $\mathrm{Rb}-\mathrm{Sr}$ ratio determination are of $1.5 \%$. Blank laboratory contamination for $\mathrm{Rb}$ is $2.5 \mathrm{ng}$ and for Sr $1.2 \mathrm{ng}$. The adopted Rb decay constant of Steiger \& Jager (1977) was used for age calculations. 


\section{Geological setting and petrography features of Monchetundra massif}

The Monchetundra intrusion belongs to Northern (Kola) belt and has a northwestwardly elongated oval shape with a total area of ca. $120 \mathrm{sq} . \mathrm{km}$ (Fig. 7). The length of the intrusion is ca. $30 \mathrm{~km}$, and the width varies from 2 to $6 \mathrm{~km}$. From east and southeast, the Monchetundra intrusion is separated from the Monchepluton intrusion by a thick mass of blastocataclasites and blastomilonites, and from west by the Viteguba-Seidozero fault. The shape of the intrusion is also compared with a lopolith. The intrusion generally plunges south-westwards, but in its central part the layering and trachytoid elements occur near-horizontally, dipping towards the axial part of the intrusion. The maximum vertical thickness of the Monchetundra intrusion cross-section exceeds $2 \mathrm{~km}$ (Fig. 7).

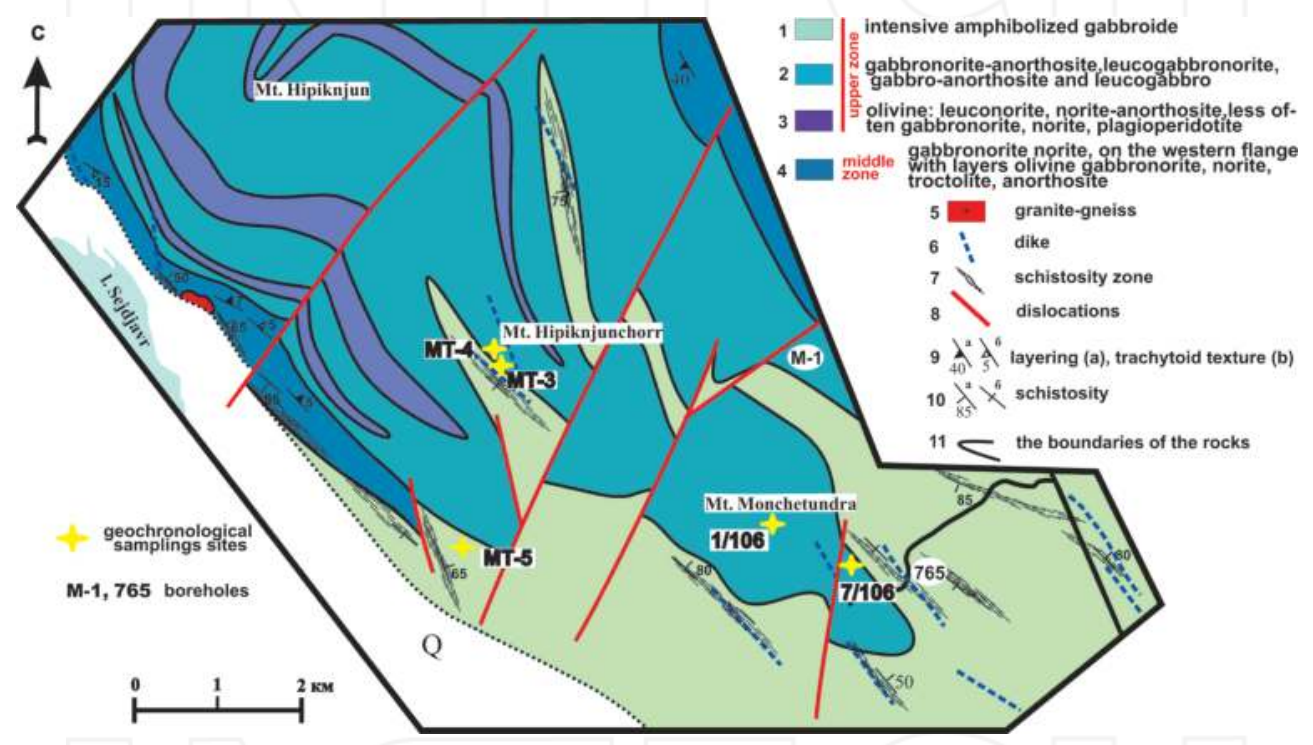

Figure 7. Schematic geological map of the central and southeastern parts of the Monchetundra intrusion (compiled by L.I. Nerovich on the materials of Central Kola Expedition OAO, Geological Institute KSC RAS with additions and amendments).

According to the results of the geological and petrographic study carried out on the principles of cumulative stratigraphy (Eules, Cawthorn, 1995; Irvine, 1982), the mafic and ultramafic rocks of the Monchetundra intrusion have been divided into three zones. The rock sequence of the lower zone (Fig. 8) varies from olivinite to leucocratic norite. The lower zone is dominated by norites with quite a wide distribution of pyroxenites and olivinites, which are coumon in the south-eastern flank of the intrusion. Minor are harzburgites and gabbronorites. Orthopyroxene and olivine cumulates prevail in the cumulative stratigraphy of the lower zone. The rocks of the middle zone represent northwestwardly elongated strings at the exposed surface of the eastern and western flanks of the intrusion (Fig. 7) and compose a significant part of the 
cross-section in the boreholes. The rock sequence of the middle zone ranges from troctolite and olivine gabbronorite to anorthosite. Contrast layering is more typical of the western flank of the intrusion. The lower zone is dominated by trachytoid medium-grained gabbronorite with plagioclase-pyroxene and minor plagioclase cumulates. The rocks of the upper zone make up the central part of the intrusion (Fig. 7).

\section{Petrographical features rocks of the Monchetundra massif}

In terms of composition, the rocks of the upper zone range from plagioperidotites to gabbronorite-anorthosites and gabbro-anorthosites. The contrast of the rocks increases northwestwards. Massive coarse-grained augite-pigeonite and augite-enstatite varieties of gabbronoriteanorthosites and leucogabbronorites, gabbro-anorthosite and leucogabbro prevail in the upper zone. The leucogabbro apparently represents an individual intrusive phase since there are xenoliths of leucocratic varieties of gabbronorites in the gabbro-anorthosites and leucogabbros of the upper and middle part of the Hipiknyunchorr Mt. slopes. The rocks of the upper zone correspond to plagioclase cumulates: poikilitic inclusions of plagioclase are typically observed in the pyroxenes and olivines.

Minor are plagioclase-pyroxene and plagioclase-olivine cumulates. Poikilitic inclusions of cumulus plagioclase in pyroxenes and olivines are found even in such melanocratic rocks as plagioperidotites of the upper zone. Thus, the lower zone of the Monchetundra intrusion consists of orthopyroxene and olivine cumulates, the middle zone of pyroxene-plagioclase and plagioclase cumulates, and the upper zone mainly of plagioclase cumulates.

Massive coarse-grained meso-leucocratic, mesocratic, and rarely melanocratic amphiboleplagioclase rocks are coumon in the southeastern and southwestern parts of the Monchetundra intrusion (Fig. 7). These rocks are mainly thought to be altered varieties of leucogabbro, gabbroanorthosite, and gabbro. Only relic clinopyroxene rarely shows well-preserved primary igneous features. The observed relic gabbro-ophytic and poikiloophytic structures indicate that the rocks are close to the leucogabbro and gabbro-anorthosite of the upper zone. However, secondary alteration of the rocks strongly hampers their investigation.

The internal structure of the Monchetundra intrusion displays significant lateral heterogeneity. The degree of differentiation tends to increase from the eastern flank of the intrusion southwards (for the lower zone) and westwards (for the middle and upper zones). This implies a possibility to find PGE mineralization not only at the junction of the Monchetundra and Monchepluton intrusions (eastern and southeastern flanks of the intrusion) that has mainly been investigated, but also in the rocks of the southwestern, western, and northwestern flanks of the intrusion.

The Monchetundra intrusion was earlier found to contain a level of noble metal mineralization confined to the norite and pyroxenite of the lower zone (Grokhovskaya et al., 2003; Smolkin et al., 2004). In 2005-2010, the exposed central and southeastern parts of the Monchetundra intrusion that are mainly composed of the upper zone rocks and of the middle zone rocks in 
the eastern and western flanks, were sampled for a geochemical analysis. A geochemical sample of $2 \mathrm{~kg}$ was taken for analysis. The rocks were analyzed for PGE, Au and Ag using atomic absorption method at the Analytic Laboratory for noble metals of the Geological Institute KSC RAS. The resultant local anomalies of noble metals were confirmed by mineragraphic methods. The polished samples were studied with scanning electron microscope LEO-1450 at the Physical Analysis Laboratory of the Geological Institute KSC RAS.

The analysis of the geochemical data has shown that the local geochemical anomalies are mainly concentrated within the western slope of Mt. Monchetundra. The only exception is Mt. Hipiknyunchorr, where increased Pd content is observed along the whole intersection from east to west. On the whole, for the massive gabbroids of the upper zone, and for the dike and veins of the intrusion, oxide mineralization that is often accompanied by syngenetic chalcopyrite and epigenetic chalcosine-bornite-chalcopyrite masses, is more typical. It correlates with a slight increase in Pd and rarely Au content. A slight increase in Pd content is also registered in the chlorite-amphibole schists after gabbroids adjacent to the shear-fault displacement planes. No noble-metal minerals themselves have been found in the above-discussed cases. Higher concentrations of valuable components and noble-metal minerals are established in the trachytoid gabbronorite of the middle zone at the western flank of the intrusion. The mineralization traced for over $10 \mathrm{~km}$ has been clearly associated with the top of the middle zone in terms of structure and lithology. It points out the stratiform character of mineralization, similar of sulphides ores at Sudbury (Li, Naldrett, 1993).

\section{Isotope - U-Pb data on zircon and baddeleyite from Monchetundra}

The age of the lower zone has about established in Pentlandite gorge (the contact zone between Monchepluton and Monchetundra). The trachytoid gabbronorite of the middle zone emplaced $-2501 \pm 8 \mathrm{Ma}$ and $2505 \pm 6 \mathrm{Ma}$ (Bayanova et al., 2010). The gabbro-anorthosite of the upper zone was previously dated to have an age of $2453 \pm 4$ Ma (Mitrofanov et al., 1993) that within error was confirmed by the investigations carried out within the present project $(2456 \pm 5 \mathrm{Ma}$, Fig. $8 \mathrm{~A}$, Table 4 ). The ages of $2471 \pm 9$ Ma and $2476 \pm 17$ Ma obtained on baddeleyite that as a primary igneous mineral allows reliably establishing the time of crystallization corroborate the geological evidence of the earlier emplacement of the upper zone leucogabbronorite and gabbronorite-anorthosite based on the xenoliths found in the gabbro-anorthosite (Fig. 8B-C, Table 4). Thus, according to the U-Pb isotope study, two injection phases have been established for the upper zone of the Monchetundra intrusion: an earlier (2471 $\pm 9 \mathrm{Ma}, 2476 \pm 17 \mathrm{Ma})$ and a later $(2456 \pm 5 \mathrm{Ma}, 2453 \pm 4 \mathrm{Ma})$ one. The ages of $2420 \pm 5 \mathrm{Ma}$ (Fig. 8D, Table 4 ) indicate the time of the earliest rock alterations and within error coincides with the alteration age of the other Early Proterozoic gabbro-anorthosite intrusions (Mitrofanov et al., 1993). A discordant age of $2521 \pm 8 \mathrm{Ma}$ (Fig. $8 \mathrm{E}$, Table 4), however, may indicate the presence of rocks of different age among strongly amphibolized gabbroids. The range of ages for the Monchetundra intrusion confirms its polychronous nature and long-term evolutional history, and is close to that for the Fedorovo-Pansky Complex (Balashov et al., 1993; Mitrofanov et al., 2005; Bayanova et al., $2009,2010)$. 

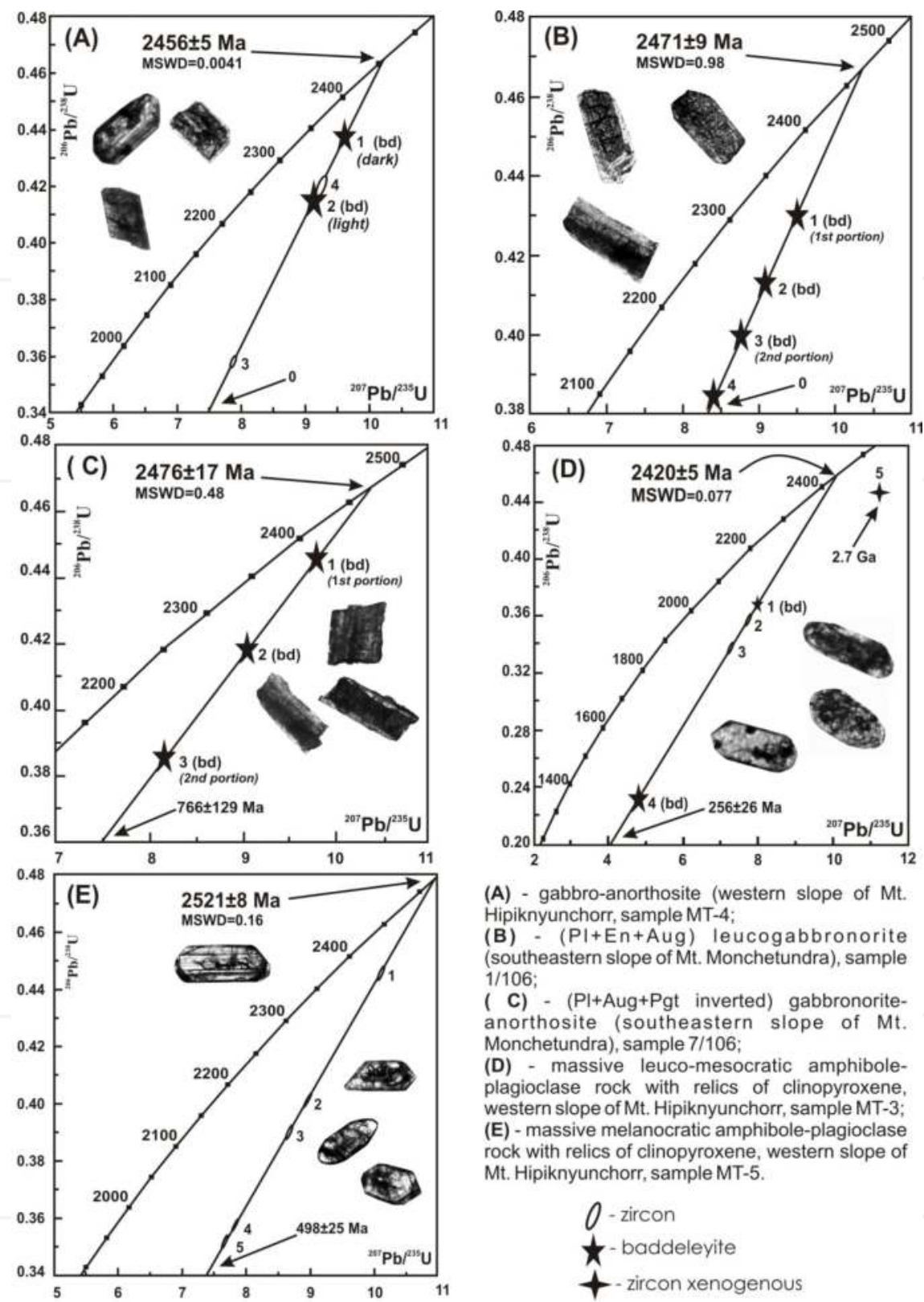

(A) - gabbro-anorthosite (western slope of Mt. Hipiknyunchorr, sample MT-4;

(B) - (PI+En+Aug) leucogabbronorite (southeastern slope of Mt. Monchetundra), sample 1/106;

( C) - (PI+Aug+Pgt inverted) gabbronoriteanorthosite (southeastern slope of Mt. Monchetundra), sample 7/106;

(D) - massive leuco-mesocratic amphiboleplagioclase rock with relics of clinopyroxene, western slope of Mt. Hipiknyunchorr, sample MT-3;

(E) - massive melanocratic amphibole-plagioclase rock with relics of clinopyroxene, western slope of Mt. Hipiknyunchorr, sample MT-5.

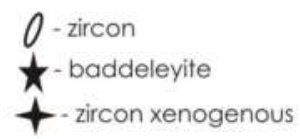

Figure 8. U-Pb concordia diagrams for zircon, baddeleyite and rutile from different rocks of the Monchegorsk Layered Complex. 


\begin{tabular}{|c|c|c|c|c|c|c|c|c|c|c|}
\hline \multirow[t]{3}{*}{ No. } & \multirow[t]{3}{*}{$\begin{array}{l}\text { Weight of portion, } \\
\text { Mg }\end{array}$} & \multicolumn{2}{|c|}{$\begin{array}{c}\text { Concentration, } \\
\text { ppm }\end{array}$} & \multicolumn{3}{|c|}{$\mathrm{Pb}$ isotope composition } & \multicolumn{3}{|c|}{ Isotope ratios and age $\mathrm{Ma**}$} & \multirow[t]{3}{*}{ Rho } \\
\hline & & \multirow{2}{*}{$\mathrm{Pb}$} & \multirow{2}{*}{$U$} & ${ }^{206} \mathrm{~Pb} /$ & ${ }^{206} \mathrm{~Pb} /$ & ${ }^{206} \mathrm{~Pb} /$ & ${ }^{207} \mathrm{~Pb} /$ & ${ }^{206} \mathrm{~Pb} /$ & ${ }^{207} \mathrm{~Pb} /$ & \\
\hline & & & & ${ }^{204} \mathrm{~Pb}$ & ${ }^{207} \mathrm{~Pb}$ & ${ }^{208} \mathrm{~Pb}$ & ${ }^{235} \mathrm{U}$ & $238 \mathrm{U}$ & ${ }^{206} \mathrm{~Pb}$ & \\
\hline \multicolumn{11}{|c|}{ Coarse-grained massive gabbro-anorthosite, slightly amphibolized, western slope of Mt. Khippiknyunchorr (MT-4) } \\
\hline 1 & $0.30(\mathrm{bd})$ & 62.2 & 138.3 & 3759 & 6.1220 & 35.850 & 9.64313 & 0.437144 & 2456 & 0.97 \\
\hline 2 & $0.10(b d)$ & 45.5 & 107.9 & 4982 & 6.1556 & 48.241 & 9.12187 & 0.413717 & 2455 & 0.95 \\
\hline 3 & $0.50(\mathrm{zr})$ & 348.4 & 634.6 & 7223 & 6.191 & 1.6275 & 7.88063 & 0.357735 & 2453 & 0.84 \\
\hline \multirow[t]{2}{*}{4} & $0.20(z r)$ & 216.8 & 420.0 & 562 & 5.7009 & 5.5664 & 9.06935 & 0.430759 & 2376 & 0.78 \\
\hline & \multicolumn{9}{|c|}{ Medium-to-coarse-grained leucogabbronorite, southeastern slope of Mt. Monchetundra (1/106) } & \\
\hline 1 & $0.50(\mathrm{bd})$ & 110.7 & 244.9 & 1478 & 5.9187 & 23.690 & 9.504470 & 0.429716 & 2460 & 0.94 \\
\hline 2 & $0.35(\mathrm{bd})$ & 152.6 & 359.1 & 3510 & 6.1640 & 35.011 & 9.119096 & 0.413367 & 2441 & 0.95 \\
\hline 3 & $0.50(\mathrm{bd})$ & 60.5 & 136.8 & 830 & 5.5615 & 13.663 & 8.820570 & 0.400447 & 2453 & 0.91 \\
\hline 4 & $0.20(\mathrm{bd})$ & 98.0 & 246.4 & 1539 & 6.3461 & 24.580 & 8.368770 & 0.382377 & 2437 & 0.83 \\
\hline
\end{tabular}

\begin{tabular}{|c|c|c|c|c|c|c|c|c|c|c|}
\hline \multicolumn{11}{|c|}{ Medium-to-coarse-grained gabbronorite-anorthosite, southeastern slope of Mt. Monchetundra (7/106) } \\
\hline 1 & $0.25(\mathrm{bd})$ & 94.5 & 114.8 & 86 & 3.3003 & 2.2134 & 9.92226 & 0.450763 & 2500 & 0.70 \\
\hline 2 & $0.20(\mathrm{bd})$ & 57.6 & 123.1 & 570 & 5.5602 & 11.459 & 9.08165 & 0.417879 & 2430 & 0.93 \\
\hline 3 & $0.25(\mathrm{bd})$ & 30.1 & 67.9 & 557 & 5.6496 & 8.0718 & 8.19067 & 0.385383 & 2392 & 0.88 \\
\hline \multicolumn{11}{|c|}{ Medium-to-coarse-grained massive metamorphosed leucogabbro, western slope of Mt. Khippiknyunchorr (MT-3) } \\
\hline 1 & $0.35(\mathrm{bd})$ & 145.6 & 385.9 & 9943 & 6.6849 & 24.354 & 7.82654 & 0.368090 & 2326 & 0.94 \\
\hline 2 & $0.40(z r)$ & 132.2 & 245.9 & 2892 & 6.4701 & 1.7904 & 7.68813 & 0.362228 & 2347 & 0.91 \\
\hline 3 & $0.25(\mathrm{zr})$ & 296.5 & 590.4 & 10687 & 6.4780 & 1.7975 & 7.18160 & 0.340041 & 2382 & 0.94 \\
\hline 4 & $0.20(\mathrm{bd})$ & 22.9 & 96.0 & 3075 & 6.4669 & 27.545 & 4.69641 & 0.232147 & 2351 & 0.86 \\
\hline 5 & $0.15(z r)$ & 38.3 & 73.6 & 3640 & 5.2862 & 7.1653 & 11.6136 & 0.453251 & 2706 & 0.96 \\
\hline \multicolumn{11}{|c|}{ Medium-to-coarse-grained massive metamorphosed gabbro, western slope of Mt. Khippiknyunchorr (MT-5) } \\
\hline 1 & $0.20(z r)$ & 442.9 & 674.4 & 11730 & 6.1317 & 1.3673 & 8.96466 & 0.401310 & 2477 & 0.95 \\
\hline 2 & $0.20(z r)$ & 110.7 & 187.1 & 4750 & 6.0548 & 1.6944 & 8.67071 & 0.389643 & 2482 & 0.85 \\
\hline 3 & $0.30(z r)$ & 717.3 & 1260.8 & 8770 & 6.1474 & 1.4718 & 7.83127 & 0.357492 & 2469 & 0.96 \\
\hline 4 & $0.20(z r)$ & 238.8 & 453.0 & 6557 & 6.2417 & 1.7384 & 7.67200 & 0.351541 & 2437 & 0.9 \\
\hline
\end{tabular}

Notes: ratios corrected to the free-running contamination $0.08 \mathrm{ng}$ for $\mathrm{Pb}$ and $0.04 \mathrm{ng}$ for $\mathrm{U}$ and to mass-discrimination of $0.12 \pm 0.04 \%$. ${ }^{* *}$ Correction to admixture of coumon lead made for the age acc. to the model by Stacey and Kramers (Stacey, Kramers, 1975).

Table 4 Isotope U-Pb (ID-TIMS) data for baddeleyite (bd) and zircon (zr) from the rocks of the Monchetundra intrusion. 


\section{Pentlandite gorge (Monchetundra and Monchepluton): Isotope $\mathrm{U}-\mathrm{Pb}$ age on single zircon and baddeleyite and Sm-Nd data on rock-forming and sulfides minerals from plagiopyroxenites}

Pentlandite gorge is a very important part of Monchetundra massif so it is a contact place with Monchegorsk intrusions (Smolkin et al., 2004). From plagiopyroxenites of outcrops about 80 $\mathrm{kg}$ were separated accessory minerals-baddeleyite and zircon were very small sizes about 50-70 $\mu \mathrm{m}$ and about 1-2 mg. Baddeleyite grains are black and dark brown were separated by 2 types due to sizes. Zircon grains are brown and were a fragment of the crystals and subdivided onto 4 types on sizes. Isotope U-Pb dating presented in (Fig. 9A, Table 5) and all 6 points lie near the concordant with age 2502.3 \pm 5.9 Ma which reflect origin of plagiopyroxenite.

The final magmatic activity in Monchetundra massif connected with gabbro pegmatite rocks. About $50 \mathrm{~kg}$ of the rocks were taken for $\mathrm{U}-\mathrm{Pb}$ single zircon dating. All zircon from separation procedures (Bayanova et al., 2009) were subdivided into 3 types due to colored. Grains of zircon are pink and have slightly rounded and corroded features with $100 \mu \mathrm{m}$ in sizes. All zircon population in CL transmission are characterized by invisible zonetions and was separated into intensively of color on three types to UOPb dating. Single zircon from gabbro-pegmatite on $\mathrm{U}$ $\mathrm{Pb}$ isochron yielded 2445.1 $\pm 1.7 \mathrm{Ma}$ and reflect the age of crystallization of the rock (Fig. 9B, Table 5).
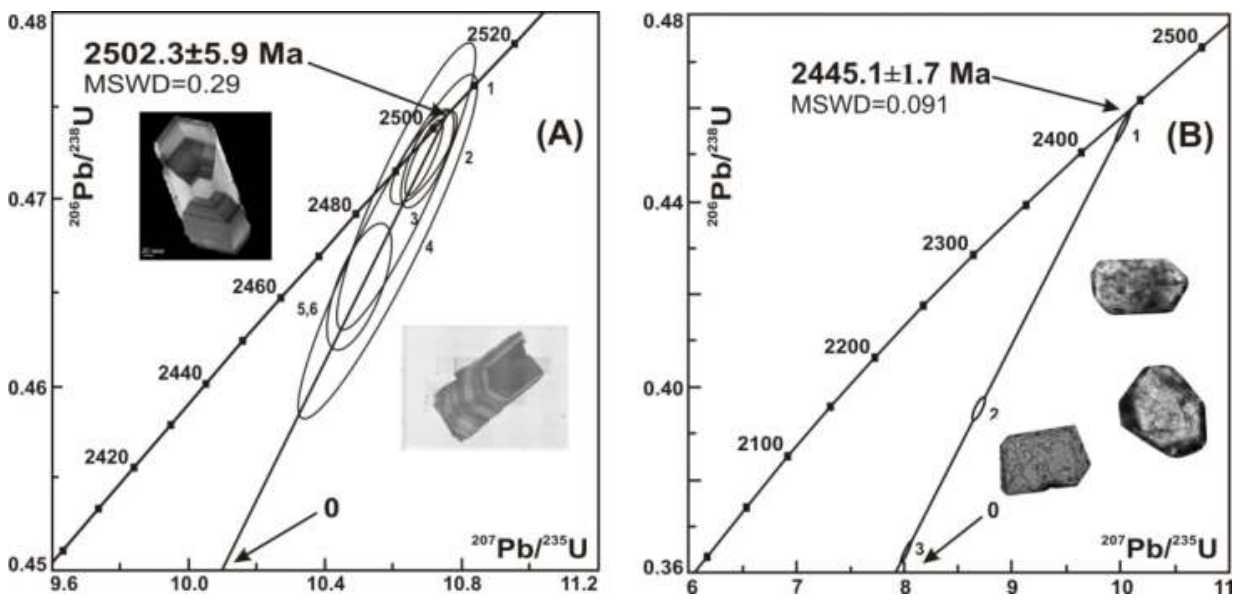

Figure 9. U-Pb concordia diagrams from Pentlandite gorge (Monchetundra massif) on: a-zircon (1-3, 6) and baddeleyite $(4,5)$ from plagiopyroxenite; b-zircon from gabbro-pegmatite. 
From plagiopyroxenites of Pentlandite gorge were separated rock-forming minerals (Cpx and Opx, Pl) and sulfides (Po) to Sm-Nd dating. All 6 minerals yielded Sm-Nd isochron age with $2489 \pm 49 \mathrm{Ma}$ (Fig. 10, Table 6) with positive $\varepsilon_{\mathrm{Nd}}= \pm 1.2$ and (model $\mathrm{T}_{\mathrm{DM}}$ age $-3192 \mathrm{Ma}$ ). Two points of sulphides - mixture of sulfides and pyrrhotine together with rock-forming minerals have shown less error in Sm-Nd dating and possibilities using sulphides in dating of layered PGE intrusions (Yekimova et al., 2011). Value $\varepsilon_{\mathrm{Nd}}(\mathrm{T})$ is positive and considered as a result of depleted mantle reservoir $(\mathrm{DM})$.

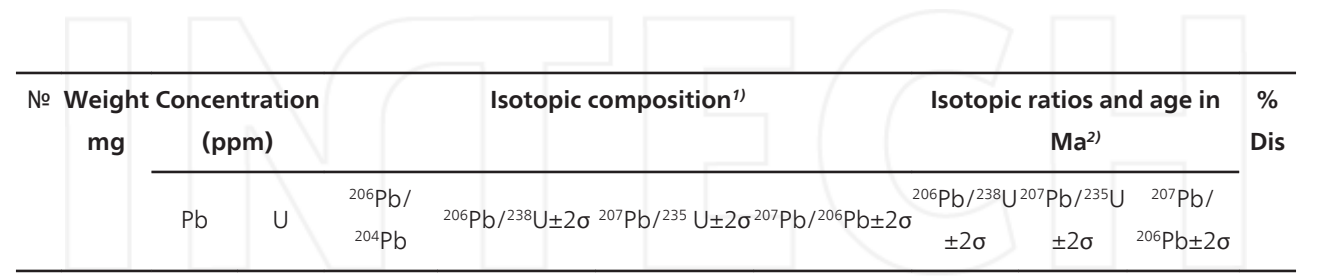

Plagiopyroxenite, zircon (1-3, 6) and baddeleyite $(4,5)$

\begin{tabular}{|c|c|c|c|c|c|c|c|c|c|c|}
\hline 1 & 0.099 & 25.45 & 38.49 & 1056.1 & $0.473 \pm 0.002$ & $10.713 \pm 0.059$ & $\begin{array}{c}0.1644 \pm 0.000 \\
6\end{array}$ & $2496 \pm 112499 \pm 14$ & $2501 \pm 8$ & 0.2 \\
\hline 2 & 0.093 & 25.46 & 30.49 & 975.1 & $0.472 \pm 0.002$ & $10.687 \pm 0.059$ & $\begin{array}{c}0.1642 \pm 0.000 \\
6\end{array}$ & $2493 \pm 112496 \pm 14$ & $2499 \pm 9$ & 0.2 \\
\hline 3 & 0.085 & 43.19 & 68.43 & 1084.9 & $0.472 \pm 0.002$ & $10.684 \pm 0.049$ & $\begin{array}{c}0.1643 \pm 0.000 \\
4\end{array}$ & $2491 \pm 102496 \pm 11$ & $2500 \pm 6$ & 0.4 \\
\hline 4 & 0.010 & 60.65 & 67.39 & 257.0 & $0.472 \pm 0.008$ & $10.683 \pm 0.188$ & $\begin{array}{c}0.1640 \pm 0.001 \\
0\end{array}$ & $2493 \pm 40 \quad 2496 \pm 44$ & $2499 \pm 18$ & 0.2 \\
\hline 5 & 0.010 & 60.07 & 69.55 & 414.1 & $0.467 \pm 0.007$ & $10.588 \pm 0.178$ & $\begin{array}{c}0.1640 \pm 0.001 \\
0\end{array}$ & $2473 \pm 392488 \pm 42$ & $2500 \pm 16$ & 1.1 \\
\hline 6 & 0.076 & 271.47 & 286.32 & 859.2 & $0.465 \pm 0.003$ & $10.509 \pm 0.086$ & $\begin{array}{c}0.1638 \pm 0.000 \\
9\end{array}$ & $2463 \pm 152481 \pm 20$ & $2495 \pm 14$ & 1.3 \\
\hline & & & & & Gabbro & -pegmatite, zir & rcon & & & \\
\hline 1 & 0.15 & 61.20 & 96.42 & 1709.4 & $\begin{array}{c}0.4562 \pm 0.003 \\
3\end{array}$ & $10.003 \pm 0.074$ & $\begin{array}{c}0.1590 \pm 0.000 \\
3\end{array}$ & $2423 \pm 18 \quad 2435 \pm 18$ & $2445 \pm 4$ & 0.9 \\
\hline 2 & 0.05 & 130.84 & 225.37 & 1594.9 & $\begin{array}{c}0.3953 \pm 0.002 \\
9\end{array}$ & $8.678 \pm 0.080$ & $\begin{array}{c}0.1592 \pm 0.000 \\
9\end{array}$ & $2147 \pm 16 \quad 2304 \pm 21$ & $2447 \pm 14$ & 12.2 \\
\hline 3 & 0.08 & 10.31 & 28.13 & 460.6 & $\begin{array}{c}0.3650 \pm 0.003 \\
1\end{array}$ & $8.011 \pm 0.070$ & $\begin{array}{c}0.1599 \pm 0.000 \\
3\end{array}$ & $1361 \pm 121846 \pm 16$ & $2447 \pm 5$ & 44.4 \\
\hline
\end{tabular}

${ }^{11}$ The ratios are corrected for blanks of $1 \mathrm{pg}$ for $\mathrm{Pb}$ and $10 \mathrm{pg}$ for $\mathrm{U}$ and for mass discrimination $0.12 \pm 0.04 \%$.

${ }^{2)}$ Correction for common $\mathrm{Pb}$ was determined for the age according to Stacey and Kramers (1975).

Table 5 Isotope U-Pb data on zircon and baddeleyite from different rocks of Monchetundra massif. 


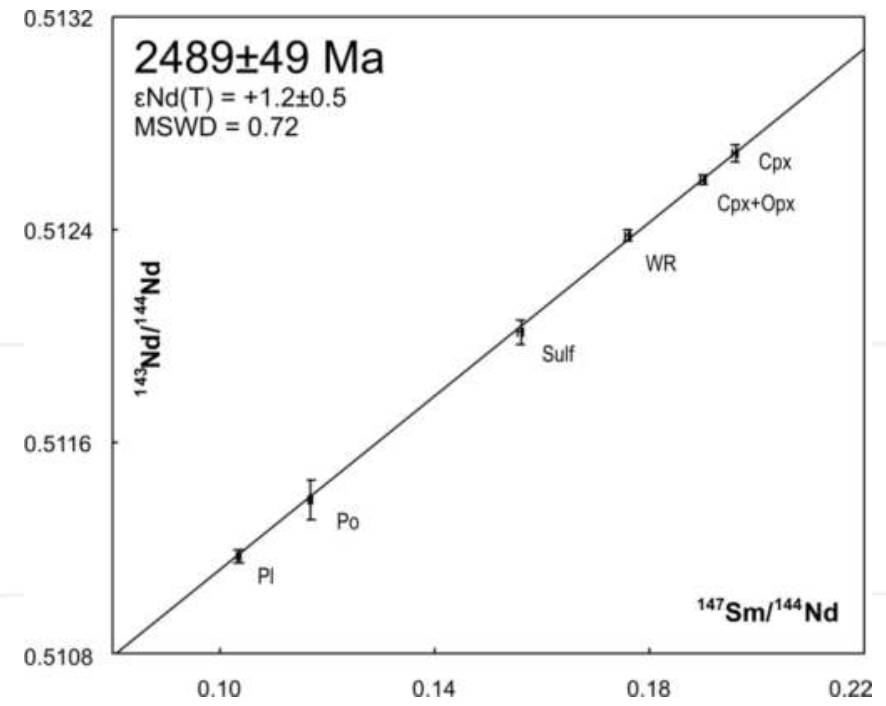

Figure 10. Isotope Sm-Nd mineral isochrone for rock-forming and sulphides minerals from plagiopyroxenites of Pentlandite gorge.

\begin{tabular}{|c|c|c|c|c|c|c|}
\hline \multirow{2}{*}{$\begin{array}{l}\text { Sample } \\
\text { № }\end{array}$} & \multicolumn{2}{|c|}{$\begin{array}{l}\text { Concentration } \\
\text { (ppm) }\end{array}$} & \multicolumn{2}{|c|}{ Isotope ratios } & \multirow[t]{2}{*}{$\mathrm{T}_{\mathrm{DM}}(\mathrm{Ga})$} & \multirow[t]{2}{*}{$\varepsilon_{\mathrm{Nd}}(2.5 \mathrm{Ga})$} \\
\hline & $\mathrm{Sm}$ & $\mathrm{Nd}$ & ${ }^{147} \mathrm{Sm} /{ }^{144} \mathrm{Nd}$ & ${ }^{143} \mathrm{Nd} /{ }^{144} \mathrm{Nd}$ & & \\
\hline P-1/109 WR & 0.678 & 2.090 & 0.17617 & $0.512377 \pm 19$ & 3192 & \pm 1.2 \\
\hline P-1/109 Po & 0.018 & 0.095 & 0.11710 & $0.011381 \pm 59$ & & \\
\hline P-1/109 Sulf & 0.032 & 0.123 & 0.15607 & $0.512015 \pm 43$ & & \\
\hline P-1/109 Cpx & 1.048 & 3.230 & 0.19614 & $0.512687 \pm 33$ & & \\
\hline P-1/109 Оpx \pm Сpx & 1.095 & 3.374 & 0.19018 & $0.512586 \pm 16$ & & \\
\hline P-1/109 PI & 0.090 & 0.523 & 0.10363 & $0.511171 \pm 26$ & & \\
\hline
\end{tabular}

Average standard values: $\mathrm{N}=11$ (La Jolla:=0.5118336); N=100 (JNdi1:=0.51209815).

Table 6 Isotope Sm-Nd data on rock-forming and sulphides minerals from Pentlandite gorge.

\section{Specific features of the isotope investigation of the intrusions}

$\mathrm{U}-\mathrm{Pb}$ (TIMS), Sm-Nd, and $\mathrm{Rb}-\mathrm{Sr}$ methods have been applied in this work for different purposes. 
The $\mathrm{U}-\mathrm{Pb}$ concordia and isochron method has been used to define the age of the rocks. The values obtained on zircons and baddeleyites from the same sample usually lie at the isochron (Table 7), indicating a similar age of magma crystallization and subsequent transformations. Coordinates of baddeleyites are near the concordia line. The method however encounters the obstacle that mafic rocks contain very few zircon and baddeleyite grains. Samples of tens of kilograms yield only a few milligrams of these minerals.

The Sm-Nd system is not an accurate geochronometer ( 2-5\%). However, the Sm-Nd isochron method can allow the establishment of crystallization times for mafic rocks on major rockforming minerals (orthopyroxene, clinopyroxene, and plagioclase). It is especially important for dating rocks with syngenetic ore minerals and sulphides. For example, this method has been used to determine for the first time the age $(2426 \pm 38 \mathrm{Ma})$ of the early ore body in the Penikat Cu-Ni-PGE deposit of the Finland (Southern belt) that has economic importance (Yekimova et al., 2011).

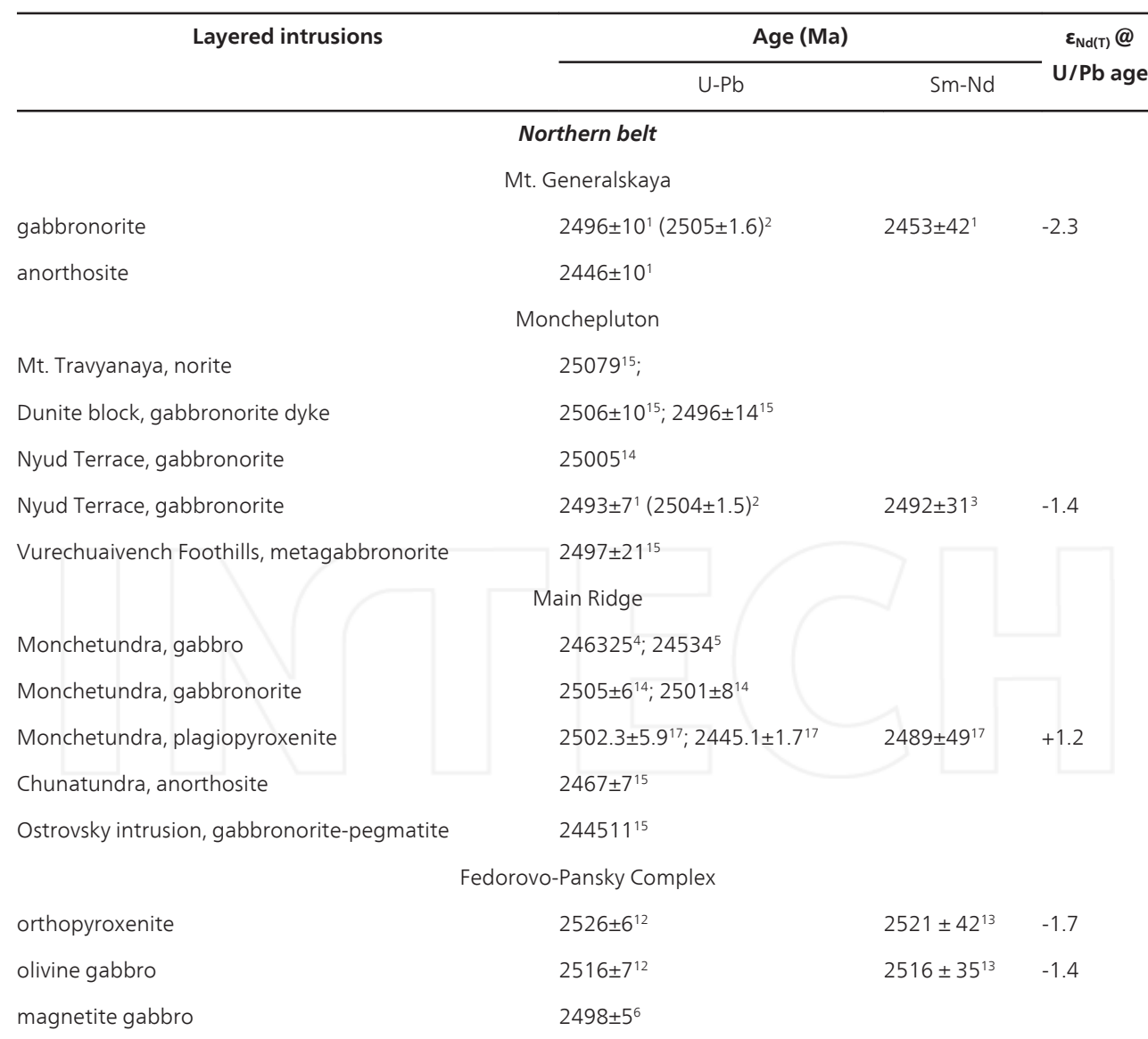




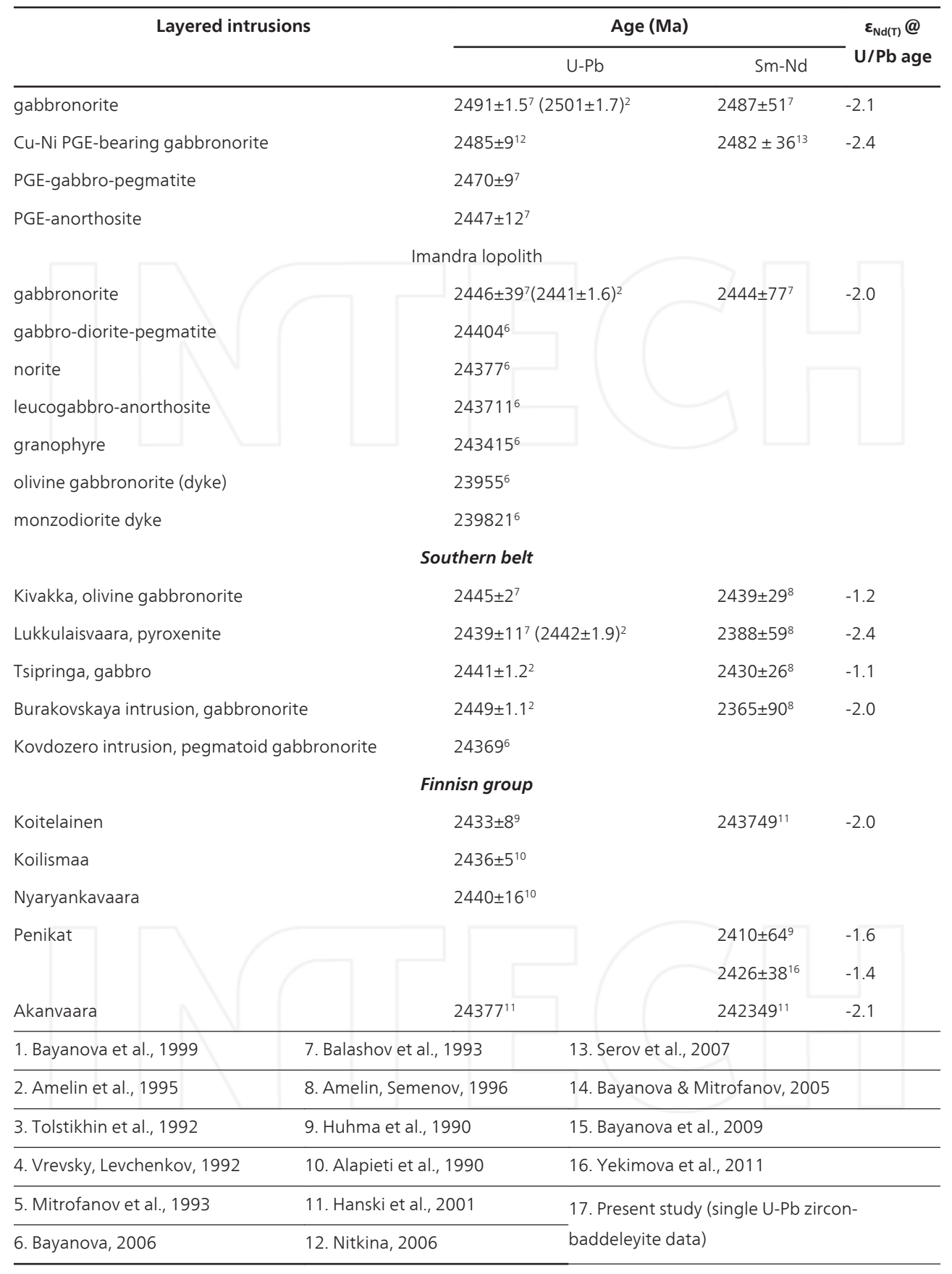

Table 7 Summary of U-Pb and Sm-Nd geochronology for layered intrusions located in the eastern Baltic Shield. 
For the mafic-ultramafic intrusions of the Kola belt, the Sm-Nd ages overlap because of high errors, but are commonly close to the U-Pb (TIMS) data on zircon and baddeleyite.

\begin{tabular}{|c|c|c|c|c|c|c|c|c|}
\hline Sample & Conce & tration & Isotopic rati & & $\varepsilon_{\mathrm{Nd}}$ & $T_{D M}$ & ${ }^{87} \mathrm{Rb} /{ }^{86} \mathrm{Sr}$ & ${ }^{87} \mathrm{Sr} /{ }^{86} \mathrm{Sr}( \pm 2 \sigma)$ \\
\hline \multirow[t]{2}{*}{ No } & \multicolumn{4}{|l|}{ (ppm) } & \multirow[t]{2}{*}{ (2.5Ga) } & \multirow{2}{*}{\multicolumn{2}{|c|}{ (Ga) }} & \multirow[t]{2}{*}{ @2.5 Ga } \\
\hline & Sm & $\mathrm{Nd}$ & ${ }^{147} \mathrm{Sm} /{ }^{144} \mathrm{Nd}$ & $\begin{array}{l}{ }^{143} \mathrm{Nd} /{ }^{144} \mathrm{Nd} \\
( \pm 2 \sigma)\end{array}$ & & & & \\
\hline \multicolumn{9}{|l|}{ Monchetundra } \\
\hline $\begin{array}{l}\text { MT-10, medium- } \\
\text { grained pyroxenite }\end{array}$ & 0.483 & 1.913 & 0.152689 & 0.51192533 & -0.36 & 2.81 & 0.00495 & $0.7039 \pm 2$ \\
\hline \multicolumn{9}{|l|}{ Mt. Generalskaya } \\
\hline S-3464, gabbronorite & 1.147 & 5.362 & 0.129320 & $0.511449 \pm 14$ & -2.30 & 2.91 & 0.00534 & $0.7042 \pm 2$ \\
\hline \multicolumn{9}{|c|}{ Fedorovo-Pansky intrusion } \\
\hline Pan-1, gabbronorite & 0.762 & 3.293 & 0.139980 & $0.511669 \pm 7$ & -2.00 & 2.98 & 0.00135 & $0.7032 \pm 1$ \\
\hline Pan-2, gabbronorite & 0.423 & 1.662 & 0.153714 & 0.51180720 & -2.50 & 3,18 & 0.00174 & $0.7029 \pm 2$ \\
\hline F-4, olivine gabbro & 0.629 & 2.801 & 0.135695 & 0.5115488 & -1.53 & 2.94 & 0.00144 & $0.7029 \pm 2$ \\
\hline F-3, orthopyroxenite & 0.318 & 1.166 & 0.164803 & $0.512196 \pm 12$ & -1.73 & 3.05 & 0.00205 & $0.7033 \pm 2$ \\
\hline \multicolumn{9}{|l|}{ Imandra lopolith } \\
\hline 6-57, gabbronorite & 2.156 & 10.910 & 0.119130 & $0.511380 \pm 3$ & -2.00 & 2.88 & 0.00339 & $0.7046 \pm 3$ \\
\hline \multicolumn{9}{|l|}{ Monchepluton } \\
\hline M-1, quartz norite & 1.750 & 8.040 & 0.131957 & $0.511493 \pm 3$ & -1.51 & 2.91 & 0.01053 & $0.7034 \pm 9$ \\
\hline $\mathrm{H}-7$, gabbronorite & 0.920 & 4.150 & 0.134055 & $0.511537 \pm 4$ & -1.37 & 2.90 & 0.00227 & $0.7037 \pm 2$ \\
\hline
\end{tabular}

Table 8 Isotope Sm-Nd and Rb-Sr data for rocks of the layered Paleoproterozoic intrusions.

It is also important to stress that the $\mathrm{Sm}-\mathrm{Nd}$ method provides valuable petrological and geochemical markers: $\varepsilon_{\mathrm{Nd}}(\mathrm{T})$ and $\mathrm{T}_{\mathrm{DM}}$. The $\varepsilon_{\mathrm{Nd}}$ shows the degree of mantle magma source depletion, while $\mathrm{T}_{\mathrm{DM}}$ indicates an approximate age of the mantle protolith (Faure, 1986).

The $\mathrm{Rb}-\mathrm{Sr}$ whole rock and mineral isochron method is mostly valuable for dating unaltered felsic igneous rocks and metamorphic amphibolite-facies associations (Faure, 1986). In this work, in Rb-Sr isotope values for the rocks (Fig. 11, Table 8) are considered to have only a petrological implication. Together with specific trace elements $\left(\mathrm{Cu}, \mathrm{Ni}, \mathrm{Ti}, \mathrm{V}\right.$, and LREE), $\varepsilon_{\mathrm{Nd}}$ (2.5 Ga), REE, $\varepsilon_{\mathrm{Nd}-\mathrm{I}} \mathrm{I}_{\mathrm{Sr}}$ (Fig. 11, Table 8), and ${ }^{4} \mathrm{He} /{ }^{3} \mathrm{He}$ (Table 9) data, the values of initial ${ }^{87} \mathrm{Sr} /{ }^{86} \mathrm{Sr}$ $\left(\mathrm{I}_{\mathrm{S}}, 2.5 \mathrm{Ga}\right)$ indicate an enriched mantle reservoir 2.5 billion years ago which is comparable with the modern EM-I (Hofmann, 1997). 


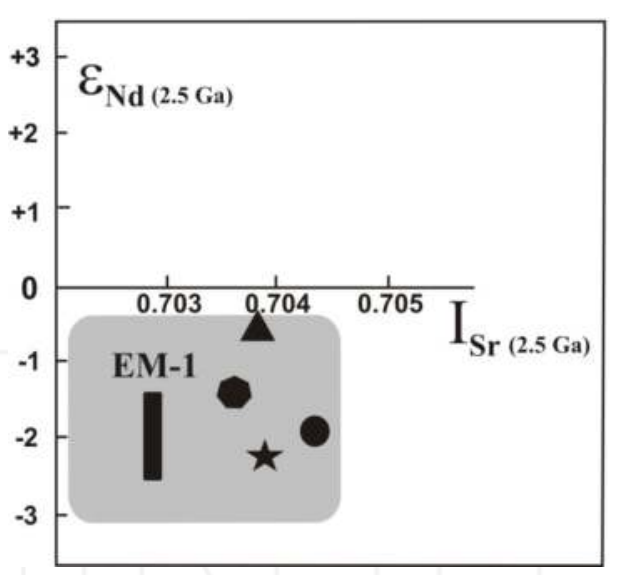

\section{- Fedorovo-Pansky Complex (Pan-1, Pan-2, F-3, F-4)}

$\star$ - Mt.Generalskaya (S-3464)

- Imandra lopolith (6-57)

- Monchetundra (MT-10)

- Monchepluton (M-1, H-7)

Figure 11. Isotope $\varepsilon_{\mathrm{Nd}-\mathrm{Sr}} \mathrm{I}_{\mathrm{sr}}$ plot of rocks from the Northern (Kola) Belt layered intrusions. Grey colour in the diagram shows EM-1 reservoir plotted for the layered intrusions of the Kola Peninsula based on the Sm-Nd and Rb-Sr isotope data given in Table 8.

\begin{tabular}{|c|c|c|c|c|}
\hline Hole No/sampling depth (m) & Rock, mineral & $\begin{array}{l}{ }^{4} \mathrm{He}^{10^{-6}} \\
\mu \mathrm{cm}^{3} / \mathrm{g}\end{array}$ & ${ }^{4} \mathrm{He} /{ }^{3} \mathrm{He} 10^{6}$ & $\begin{array}{l}\text { Low Mantle } \\
\text { contribution**\% }\end{array}$ \\
\hline \multicolumn{5}{|l|}{ Monchetundra } \\
\hline hole, $765 / 905,9$ & Clinopyroxene & 163.00 & 4.76 & 0.21 \\
\hline hole, 765/905,9 & Orthopyroxene & 21.00 & 4.76 & 0.21 \\
\hline hole, $765 / 985,3$ & Amphibole & 97.00 & 4.76 & 0.21 \\
\hline hole, 765/985,3 & Clinopyroxene & 115.00 & 5.00 & 0.20 \\
\hline outcrop, MT-5 & Gabbro & 1.30 & 2.00 & 0.41 \\
\hline \multicolumn{5}{|l|}{ Fedorovo-Pansky intrusion } \\
\hline hole, Ki-16/6 & Amphibole & 81.00 & 9.10 & 0.11 \\
\hline hole, Ma-14/1 & Orthopyroxene & 9.90 & 12.80 & 0.08 \\
\hline outcrop, No 9 & Ilmenite & 43.90 & 16.50 & 0.06 \\
\hline \multicolumn{5}{|l|}{ Monchepluton, Mt. Sopcha } \\
\hline hole, 995/315 & Olivinite, rock & 17.00 & 6.25 & 0.16 \\
\hline hole, 995/315 & Olivine & 25.00 & 5.88 & 0.17 \\
\hline hole, 995/315 & Orthopyroxene & 31.00 & 6.25 & 0.16 \\
\hline hole, 995/315 & Plagioclase & 47.00 & 5.56 & 0.18 \\
\hline hole, 995/315 & Magnetite & 132.00 & 4.35 & 0.23 \\
\hline \multicolumn{5}{|l|}{ Monchepluton, dunite block } \\
\hline hole, 904/102 & Dunite, rock & 218.00 & 1.47 & 0.68 \\
\hline hole, 904/102 & Olivine & 115.00 & 1.35 & 0.74 \\
\hline hole, $1651 / 244,9$ & Chromitite, ore & 56.00 & 1.43 & 0.70 \\
\hline hole, C-1651/373,5* & Dunite-Bronzitite & 28.00 & 0.83 & 1.20 \\
\hline hole, C-1622/7* & Chromitite, ore & 2.80 & 0.69 & 1.44 \\
\hline
\end{tabular}




\begin{tabular}{|c|c|c|c|c|}
\hline Hole No/sampling depth (m) & Rock, mineral & $\begin{array}{l}{ }^{4} \mathrm{He}^{10^{-6}} \\
\mu \mathrm{cm}^{3} / \mathrm{g}\end{array}$ & ${ }^{4} \mathrm{He} /{ }^{3} \mathrm{He} 10^{6}$ & $\begin{array}{l}{ }_{6} \text { Low Mantle } \\
\text { contribution**\% }\end{array}$ \\
\hline hole, C-1646/450* & Dunite & 2.20 & 1.29 & 0.77 \\
\hline hole, C-1651/373.5* & Dunite-Bronzitite-contact & 0.13 & 0.60 & 1.68 \\
\hline
\end{tabular}

Note: errors are according to the calculation method (Tolstikhin and Marty, 1998).

*Step wise heating experiment (fraction under the temperature 1300C).

**Mantle components are given from value ${ }^{4} \mathrm{He} /{ }^{3} \mathrm{He} 0.55 \times 10^{4}$ (solar helium from lower mantle reservoir), Tolstikhin and Marty (1998).

Table 9 Isotopic ${ }^{4} \mathrm{He} /{ }^{3} \mathrm{He}$ ratios of PGE layered intrusions of the Baltic Shield.

\section{Summary and conclusions}

\subsection{Total duration of magmatic activity, timing and multi-stages}

The largest and richest ore deposits of the Monchepluton, Monchetundra and FedorovoPansky Complexes have been carefully studied by geochronological methods.

The layered or differentiated series of mafic-ultramafic rocks, from troctolite to leucogabbroanorthosite, and syngenetic Cu-Ni-PGE ores of the Monchepluton formed within the time interval of 2516 ( $\max$ ) to 2476 ( $\mathrm{min}$ ) Ma. Without analytical errors, the time interval is from 2507 Ma to 2493 Ma. Some researchers (Smolkin et al., 2004) suggest that the Vurechuaivench part of the pluton, composed of gabbroids and anorthosites containing PGE deposits, is an independent magma chamber and that the age of rock and syngenetic PGE ore emplacement is $2497 \pm 21 \mathrm{Ma}$.

The Fedorov Block of the Fedorovo-Pansky Complex represents an independent magma chamber, the rocks and ores of which differ significantly from those of the Western Pansky Block (Schissel et al., 2002; Nitkina, 2006; Serov et al., 2007).

The early magmatic activity about 2.5 Ga manifested itself in the gabbronorite of the Monchetundra (2505 $\pm 6 \mathrm{Ma}$ and $2501 \pm 8 \mathrm{Ma}$ ) and Mt. Generalskaya (2496 $\pm 10 \mathrm{Ma})$. The magmatic activity that resulted in the formation of anorthosite took place about 2470 and $2450 \mathrm{Ma}$. It also contributed to the layered series of the Chunatundra $(2467 \pm 7 \mathrm{Ma})$ and Mt. Generalskaya (2446 $\pm 10 \mathrm{Ma})$, Monchetundra gabbro (2453 $\pm 4 \mathrm{Ma} ; 2456 \pm 5$ gabbro-anorthosite; and pegmatoid gabbronorite of the Ostrovsky intrusion ( $2445 \pm 11 \mathrm{Ma})$.

The Imandra lopolith is the youngest large layered intrusion within the Kola Belt. It varies from the other intrusions of the Kola Belt both in its emplacement age and its metallogeny. There are five U-Pb zircon and baddeleyite ages for the rocks of the main magmatic pulse represented by norite, gabbronorite, leucogabbro-anorthosite, gabbrodiorite, and granophyre; all formed within the interval from 2445 to 2434 Ma.

Thus, several eruptive pulses of magmatic activity have been established in the complex intrusions of the Kola Belt, including at least four pulses (or phases) in the Fedorovo-Pansky 
Complex: a 2526-2516 Ma barren pulse, and three ore-bearing of 2505-2485 Ma, $2470 \mathrm{Ma}$, and 2450 Ma. For similar intrusions of the Fenno-Karelian Belt, for example, Penikat intrusion in Finland, five magmatic pulses varying only in geochemistry have been distinguished from the same deep chamber (Vogel et al., 1998; Iljina \& Hanski, 2005).

A total duration for magmatic processes of over 80 million years in the Kola Belt intrusions is unexpected for many researchers.

The multi-phase magmatic duration of the Fenno-Karelian Belt intrusions was short-term and took place about $2.44 \mathrm{Ga}$ years ago. However, there are only a few $\mathrm{U}-\mathrm{Pb}$ precise age estimations for the Fenno-Karelian Belt intrusions (Iljina \& Hanski, 2005). A joint Russian-Finnish research collaboration intended for dating the intrusions of the both belts has recently been initiated. It is expected that the research will result in updating the knowledge about the timing and duration of the Paleoproterozoic ore-forming intrusions on the Baltic Shield.

The Kola results underline that the layering of the intrusions with thinly-differentiated horizons and PGE reefs was not contemporaneous (or syngenetic) with each intrusion defining its own metallogentic trends in time and space.

\subsection{Metallogeny features}

The Palaeoproterozoic magmatic activity in the eastern Baltic Shield is associated with the formation of widespread ore deposits: Cu-Ni $( \pm \mathrm{PGE}), \mathrm{Pt}-\mathrm{Pd}( \pm \mathrm{Rh}, \pm \mathrm{Cu}, \mathrm{Ni}, \mathrm{Au}), \mathrm{Cr}, \mathrm{Ti}-\mathrm{V}$ (Richardson \& Shirey, 2008; Mitrofanov \& Golubev, 2008).

On the Kola Peninsula, economic Cu-Ni $( \pm$ PGE) deposits are known in the Monchegorsk ( 2500 $\mathrm{Ma}$ ) and Pechenga ( 1980 Ma) type intrusions. In the Monchepluton (the Monchegorsk type), syngenetic disseminated Cu-Ni $( \pm$ PGE) ore bodies of magmatic origin are confined to basal parts of magmatic chambers (Papunen \& Gorbunov, 1985), while massive rich redeposited ores in the veined bodies of the Monchepluton bottom as well as beyond it (offset bodies) also contain a relatively high portion of platinum among platinum-group elements. They are associated mainly with ca. 2500 Ma magnesia-rich mafic-ultramafic rocks with $\varepsilon_{\mathrm{Nd}}(2.5 \mathrm{Ga})$ values varying from- 1 to- 2 . In comparison, $\mathrm{Cu}-\mathrm{Ni}( \pm \mathrm{PGE})$ ores of the Pechenga type intrusions that are not discussed are related to the $1980 \mathrm{Ma}$ gabbro-wehrlite rocks with $\varepsilon_{\mathrm{Nd}}(1.98 \mathrm{Ga})$ values varying from \pm 1 to \pm 3 (Hanski et al., 1990; Mitrofanov \& Golubev, 2008). The basal ores of the Fedorovo deposit are first of all valuable for platinum-group elements (Pt, Pd, Rh), but nickel, copper and gold are also of economic importance here (Schissel et al., 2002).

$\mathrm{Pt}-\mathrm{Pd}( \pm \mathrm{Cu}, \mathrm{Ni}, \mathrm{Rh}, \mathrm{Au})$ reef-type deposits and ore occurrences of the Vurechuaivench Foothills (Monchepluton) and Western Pansky Block (Fedorovo-Pansky Complex) seem, in terms of genesis, to be associated with pegmatoid leucogabbro and anorthosite rocks enriched in latestage fluids. Portions of this magma produce additional injections of ca. $2500 \mathrm{Ma}$ (Vurechuaivench), ca. $2470 \mathrm{Ma}$ (the Lower, Northern PGE reef), and ca. $2450 \mathrm{Ma}$ (the Upper, Southern PGE reef of the Western Pansky Block and PGE-bearing mineralization of the Mt. Generalskaya intrusion). These nonsimultaneous injections are quite close in terms of composition, prevalence of Pd over Pt, ore mineral composition (Mitrofanov et al., 2005), and isotope geochemistry 
of $\mathrm{Sm}-\mathrm{Nd}$ and $\mathrm{Rb}-\mathrm{Sr}$ systems. The $\varepsilon_{\mathrm{Nd}}$ values for the rocks under consideration vary from-1 to-3, which probably indicates a single long-lived magmatic hearth.

Chromium concentration (>1000 ppm) is typical geochemical feature of the lower maficultramafic rocks of the layered intrusions of the Baltic Shield (Alapieti, 1982; Iljina \& Hanski, 2005). The chromite mineralization is known in the basal series of the Monchepluton, Fedorovo-Pansky Complex, Imandra lopolith (Russia), Penikat and Narkaus intrusions (Finland) and in chromite deposits of the Kemi intrusion (Finland) and Dunite Block (Monchepluton, Russia). On the contrary, Fe-Ti-V mineralization of the Mustavaara intrusion (Finland) tends to most leucocratic parts of the layered series, and to leucogabbro-anorthosite and gabbrodiorite of the Imandra lopolith (Russia) and Koillismaa Complex (Finland).

Thus, PGE-bearing deposits of the region are represented by two types: the basal and the reeflike ones. According to modern economic estimations, the basal type of deposits is nowadays more preferable for mining, even if the PGE concentration (1-3 ppm) is lower compared to the reef-type deposits (>5ppm). Basal deposits are thicker and contain more platinum, copper and, especially, nickel. These deposits are accessible to open pit mining.

\subsection{Isotope-geochemistry and geodynamic significance}

Magmatic processes since the Palaeoproterozoic (2.53 Ga) have affected almost the whole region of the East-Scandinavian (Kola-Lapland-Karelian) province and a mature continental crust formed (2.55 Ga) in the Neoarchaean (Gorbatschev \& Bogdanova, 1993). Thick (up to 3 $\mathrm{km}$ ) basaltic volcanites of the Sumian age (2.53-2.40 Ga) in Karelia, Kola and northeast Finland cover an area of greater than $200.000 \mathrm{~km}^{2}$. In the north, magmatic analogues of these volcanic rocks are represented by two belts of layered intrusions and numerous dyke swarms (Vuollo et al., 2002, Vuollo \& Huhma, 2005). This together composes a single time-and space-related megacyclic association, the East-Scandinavian Large Igneous Province (LIP). All the magmatic units of the province covering a huge area show similar geological, compositional and metallogenic features (Coffin, Eldholm, 1994).

Regional geological settings indicate anorogenic rift-like intraplate arrangements involving volcano-plutonic belts connecting different domains of the Paleoarchaean Kola-LaplandKarelia protocontinent. This resembles early advection extensional geodynamics of passive rifting that is typical of intraplate plume processes (Pirajno, 2007).

Geochemical and isotope-geochemical data shed light on features of deep magma source for the LIP rocks. $\mathrm{T}_{\mathrm{DM}}$ values (Faure, 1986) are approximately the age of the depleted mantle reservoir (DM) with slightly enriched $\mathrm{Sm}-\mathrm{Nd}$ ratios. The $\mathrm{T}_{\mathrm{DM}}$ values lie within the interval of 3.1-2.8 Ga. The $\varepsilon_{\mathrm{Nd}}$ values vary from-1.1 to-2.4 and similar $\mathrm{I}_{\mathrm{Sr}}$ values $(0.703-0.704)$ obtained for discrete layered intrusions form a narrow range of enriched compositions. It is difficult to argue for a local crustal contamination and we suggest that the magmas producing different rocks of the LIP layered intrusions were derived from a single homogenous mantle source enriched both with typically magmatic ore elements (Ni, TI, V, and $\mathrm{Pt}$ ) and lithophile elements including light REE. To some extent, this reservoir is comparable with the modern EM-1 source (Hofmann, 1997). 
Isotope ${ }^{4} \mathrm{He} /{ }^{3} \mathrm{He}$ ratio is also a reliable isotope tracer of mantle plume processes (Tolstikhin \& Marty, 1998; Bayanova et al., 2006, 2009; Pirajno, 2007). Their use in studying Precambrian rocks and requires special case. Table 9 shows recent helium isotope data for the rocks and minerals of the Kola Belt intrusions. The data indicate that the ${ }^{4} \mathrm{He} /{ }^{3} \mathrm{He}$ isotope ratios of $\mathrm{n} \mathrm{x}$ $10^{6-5}$ correspond to those of the upper mantle and differ from those of the crust $\left(\mathrm{n} \times 10^{8}\right)$ and lower mantle ( $\left.\mathrm{n} \times 10^{4}\right)$ (Tolstikhin \& Marty, 1998). The helium isotope data tend to favour a source dominated by mantle derived magmas with only local crustal contamination.

According to the available data (Campbell, 2001; Condie, 2001; Vuollo et al., 2002; Bleeker, 2003; Ernst \& Buchan, 2003; Bleeker, Ernst, 2006; Bayanova et al., 2009; present study), the peak of the mafic-ultramafic magmatic activity of the Kola-Karelian, Superior and Wyoming provinces has been estimated at $\sim 2.45 \mathrm{Ga}$. Figure 12 presents an attempt to demonstrate some reconstruction of the Archaean supercontinent embodying these three provinces of Europe and North America (Heaman, 1997; Bleeker, Ernst, 2006; Ernst, 2008). Trends of the Kola and Fenno-Karelian Belts of 2.52-2.44 Ga layered intrusions are show with the intraplate nature interpreted from the results of the present study.

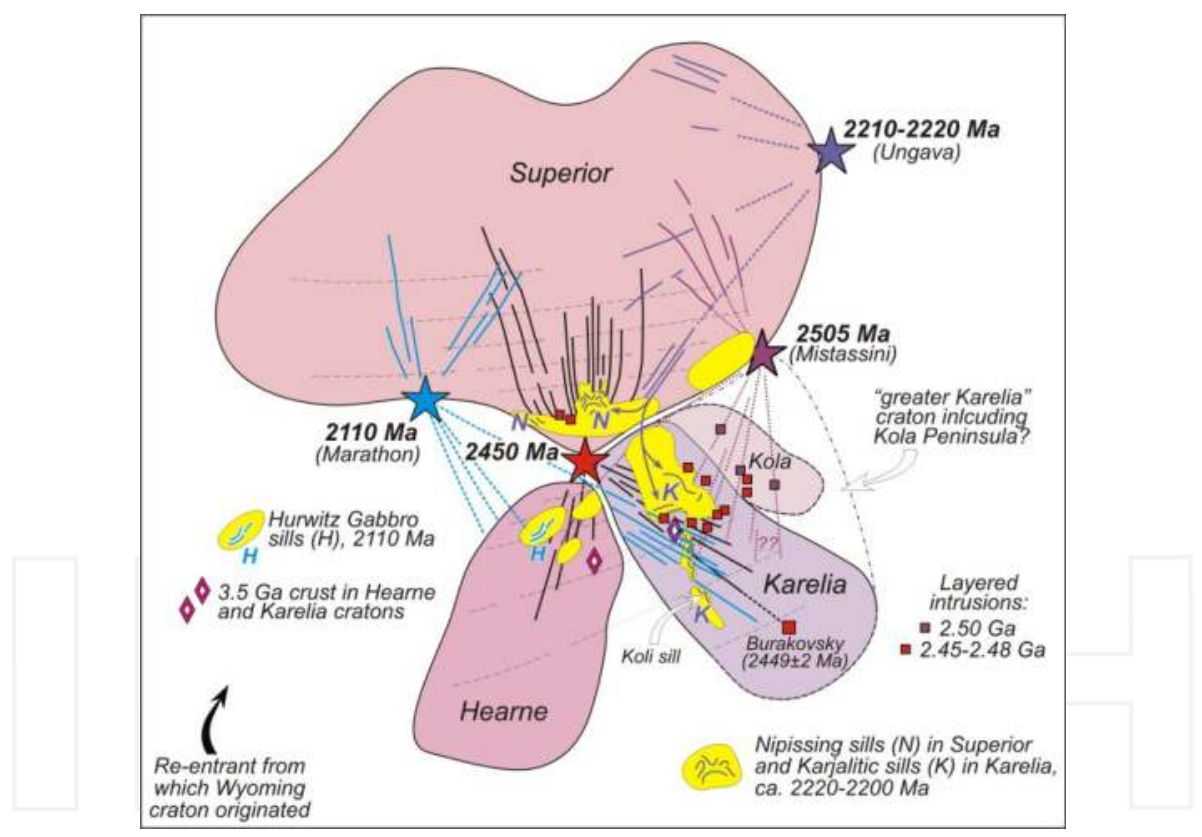

Figure 12. A new correlation for the Superior, Karelia and Hearne craton. The detailed fit is based on successful matching of several short-lived magmatic events, at ca. $2450 \mathrm{Ma}$ (Matachewan), ca. $2217 \mathrm{Ma}$ (Nipissing (N) and Karjalitic (K) sills), and ca. $2110 \mathrm{Ma}$ (Marathon), as well as correlation of the cover sequences (see text). Kola is likely part of this correlation as part of a "greater Karelia" craton. The Wyoming craton likely originated from the re-entrant west of the Hearne craton. Note that our reconstruction successfully places the ca. 3.5 Ga Siurua gneiss of Karelia (diamond symbol; "Europe's oldest rocks") along strike of similar age crust in the Hearne craton. Arrows indicate part of the long-distance transport of magma to feed the Nipissing and Karjalitic sills (Bleeker, Ernst, 2006). 
The LIP layered intrusions are directly related to the Baltic Shield metallogeny (Mitrofanov \& Golubev, 2008). The $>80 \mathrm{Ma}$ duration and multiphase history of the Kola Belt layered mafic intrusions (i.e., 2.53-to $2.45 \mathrm{Ga}$ ) has been shown here. It has also been underlined that the younger intrusions of the Fenno-Karelian Belt (Fig. 1) cluster at 2.44 Ga (Iljina \& Hanski, 2005). The partially asynchronous evolution of these two belts, that are thought to be arms of a mantle plume, is now being examined in more detail as a follow-up to this study within the framework of Russian-Finnish and Canadian research collaboration (Ernst, 2008).

A number of new $\mathrm{U}-\mathrm{Pb}$ and $\mathrm{Sm}-\mathrm{Nd}$ isotope data were obtained for Monchetundra massif of Monchegorsk ore region and various rocks of the mafic layered intrusions of the Kola Belt (Baltic Shield), including those which bear PGE, Ni-Cu and Ti-V mineralization. A surprisingly long period of multiphase magmatic activity, from 2530 to $2450 \mathrm{Ma}$ (about 80 million years), resulted in the intrusion of large-scale ore-bearing intrusions of the Kola Belt. Magmatism continued until about $2400 \mathrm{Ma}$ and generated wide-spread dykes and small-scale intrusions. These results contrast with the published data indicating short-term evolution interval ( 2440 Ma) for similar intrusions of the Fenno-Karelian Belt (Iljina \& Hanski, 2005).

The two belts of mafic layered intrusions of the Baltic Shield (the Kola and Fenno-Karelian belts), together with the surrounding volcanic rocks and dyke swarms, compose the Palaeoproterozoic East-Scandinavian Large Igneous Province (LIP) with an area of more than 200.000 $\mathrm{km}^{2}$. The petrological-geodynamic interpretation proposed by the present paper of the LIP is a product of a vast long-lived plume is based on the enriched isotope characteristics of the magmas and also the large volume and widespread distribution of the magmas. The is acknowledge that alternatives involving super-long duration of the homogenous deep-seated magma sources are possible.

\section{Discussion}

\subsection{Specific features of the isotope investigation of the intrusions}

The $\mathrm{U}-\mathrm{Pb}$ concordia and isochron method has been used to define the age of crystallization of the rocks. The values obtained on zircons and baddeleyites from the same sample usually lie at the isochron, indicating a similar age of magma crystallization and subsequent transformations. Coordinates of baddeleyites are near the Concordia line. However, the method encounters the obstacle that mafic rocks contain very few zircon and baddeleyite grains. Samples of tens of kilograms yield only a few milligrams of these minerals.

In order to compare our $\mathrm{U}-\mathrm{Pb}$ results (23 isochron points), some samples were sent to the

Royal Ontario Museum Laboratory in Canada. The data obtained there (Amelin et al. 1995) agree with ours within error.

The Sm-Nd system is not an accurate geochronometer (c. 2-5\%). However, the $\mathrm{Sm}-\mathrm{Nd}$ isochron method can allow the establishment of crystallization times for mafic rocks on major rock-forming minerals (olivine, orthopyroxene, clinopyroxene and plagioclase). It is especially 
important for dating rocks with syngenetic ore minerals. For example, this method has been used to determine for the first time the age $(2482 \pm 36 \mathrm{Ma})$ of the early ore body in the FedorovoPansky Cu-Ni-PGE deposit of the Kola Peninsula that has economic importance (Serov et al. 2007).

For the mafic-ultramafic intrusions of the Kola belt, the Sm-Nd ages overlap because of high errors, but are coumonly close to the U-Pb (TIMS) data on zircon and baddeleyite. They are especially valid for the marginal fast-crystallizing rocks of the Taxitic Zone of the FedorovoPansky Complex, where the early barren orthopyroxenite and gabbro have the following ages: $2521 \pm 42 \mathrm{Ma}$ and $2516 \pm 35 \mathrm{Ma}$ (Sm-Nd method) and 2526 $\pm 6 \mathrm{Ma}$ and $2516 \pm 7 \mathrm{Ma}$ (U-Pb method), respectively. The ore-bearing norite of the Fedorov Block yielded an age of $2482 \pm 36 \mathrm{Ma}(\mathrm{Sm}-$ $\mathrm{Nd}$ method) and $2485 \pm 9 \mathrm{Ma}$ (U-Pb method) according to Nitkina (2006) and Serov et al. (2007).

It is also important to stress that the $\mathrm{Sm}-\mathrm{Nd}$ method provides valuable petrological and geochemical markers: $\varepsilon_{\mathrm{Nd}}(\mathrm{T})$ and $\mathrm{T}_{\mathrm{DM}}$. The $\varepsilon_{\mathrm{Nd}}$ shows the degree of mantle magma source depletion, while $\mathrm{T}_{\mathrm{DM}}$ indicates an approximate age of the mantle protolith (Faure 1986).

The $\mathrm{Rb}-\mathrm{Sr}$ whole rock and mineral isochron method is mostly valuable for dating unaltered felsic igneous rocks and metamorphic amphibolitefacies associations (Faure 1986). In our work, $9 \mathrm{Rb}-\mathrm{Sr}$ isotope values for the rocks are considered to have only a petrological implication. Together with specific trace elements $\left(\mathrm{Cu}, \mathrm{Ni}, \mathrm{Ti}, \mathrm{V}\right.$ and LREE), $\varepsilon_{\mathrm{Nd}}(2.5 \mathrm{Ga})$ and ${ }^{4} \mathrm{He} /{ }^{3} \mathrm{He}$ data, the values of initial ${ }^{87} \mathrm{Sr} /{ }^{86} \mathrm{Sr}\left(\mathrm{I}_{\mathrm{Sr}}[2.5 \mathrm{Ga}]\right)$ indicate an enriched mantle reservoir 2.5 billion years ago which is comparable with the modern EM-I.

\subsection{The timing, pulsation and total duration of magmatic activity}

The largest and richest ore deposits of the Monchepluton and Fedorovo-Pansky complexes have been carefully studied by geochronological methods.

The layered or differentiated series of maficultramafic rocks, from troctolite to leucogabbroanorthosite, and syngenetic $\mathrm{Cu}-\mathrm{Ni}-\mathrm{PGE}$ ores of the Monchepluton formed within the time interval of 2516 ( $\max$ )-2476 ( $\mathrm{min}$ ) Ma. Without analytical errors, the time interval is from 25072493 Ma. Some researchers (Smolkin et al. 2004) suggest that the Vurechuaivench part of the pluton, composed of gabbroids and anorthosites containing PGE deposits, is an independent magma chamber and that the age of rock and syngenetic PGE ore emplacement is $2497 \pm 21 \mathrm{Ma}$.

The Fedorov Block of the Fedorovo-Pansky Complex represents an independent magma

chamber, the rocks and ores of which differ significantly from those of the Western Pansky Block (Schissel et al. 2002). The $2 \mathrm{~km}$-thick rock sequence, from the Marginal Zone to the Lower Gabbro Zone, is a layered or differentiated syngenetic series of relatively melanocratic pyroxenite-norite-gabbronorite-gabbro dated at $2526 \pm 6$ and $2516 \pm 7 \mathrm{Ma}$. The Taxitic Zone is penetrated by concordant and cutting $\mathrm{Cu}-\mathrm{Ni}$-PGEbearing gabbronorite (Fedorovo deposit) of the second pulse of magmatic injection, which is slightly younger (2485 \pm 9 Ma.

The Western Pansky Block from the Main Gabbronorite Zone, without the Lower Layered Horizon and probably without the upper part (above $3000 \mathrm{~m}$ ), can also be considered a single syngenetic series of relatively leucocratic, mainly olivine-free gabbronorite-gabbro crystallized 
within the interval of 2503-2498-2491 \pm 5 Ma. In the lower part of the Block there are Norite and Marginal zones. The Marginal zone contains poor disseminated $\mathrm{Cu}-\mathrm{Ni}-\mathrm{PGE}$ mineralization. This rock series can be correlated with certain parts of the Monchepluton and the Fedorov Block. The 40-80 m-thick Lower Layered Horizon (LLH) is prominent because of its contrasting structure with predominant leucocratic anorthositic rocks. The exposed part of the horizon strikes for almost $15 \mathrm{~km}$ and can be traced in boreholes down to a depth of $500 \mathrm{~m}$ (Mitrofanov et al. 2005). By its morphology, the horizon seems to be part of a single layered series. Nevertheless, there are anorthositic bodies that in outcrop show cutting contacts and apophyses (Latypov \& Chistyakova 2000); the cumulus plagioclase compositions in the rocks of the horizon are different from those in the surrounding rocks; and the age of the PGE-bearing leucogabbro-pegmatite, which is precisely defined by concordant and near-concordant $\mathrm{U}-\mathrm{Pb}$ data on zircon as $2470 \pm 9 \mathrm{Ma}$, is slightly younger than the ages of the surrounding rocks (e.g. $2491 \pm 1.5 \mathrm{Ma}$ ). The LLH rocks, especially the anorthosite and the PGE mineralization, probably represent an independent magmatic pulse.

The upper part and olivine-bearing rocks of the Western Pansky Block and the anorthosite of the Upper Layered Horizon (ULH) with the Southern PGE Reef have been poorly explored. They differ from the main layered units of the Block in rock, mineral and PGE mineralization composition (Mitrofanov et al. 2005). Until now, only one reliable U-Pb age (2447 $\pm 12 \mathrm{Ma}$ ) has been obtained for the PGE-bearing anorthosite of the block, which may represent another PGEbearing magmatic pulse.

The early magmatic activity of about 2.5 Ga manifested itself in the gabbronorite of the Monchetundra (2505 \pm 6 and $2501 \pm 8 \mathrm{Ma}$ ) and Mt Generalskaya (2496 $\pm 10 \mathrm{Ma})$. The magmatic activity that resulted in the formation of anorthosite took place about 2470 and $2450 \mathrm{Ma}$. It also contributed to the layered series of the Chunatundra (2467 $\pm 7 \mathrm{Ma})$ and Mt Generalskaya (2446 $\pm 10 \mathrm{Ma})$, Monchetundra gabbro (2453 $\pm 4 \mathrm{Ma}$, Mitrofanov et al. 1993) and pegmatoid gabbronorite of the Ostrovsky intrusion $2445 \pm 11$ Ma.

The Imandra lopolith is the youngest large layered intrusion within the Kola Belt. It varies from the other intrusions of the Kola Belt both in its emplacement age and its metallogeny. There are five $\mathrm{U}-\mathrm{Pb}$ zircon and baddeleyite ages for the rocks of the main magmatic pulse represented by norite, gabbronorite, leucogabbro-anorthosite, gabbrodiorite and granophyre; all formed within the interval from 2445-2434 Ma.

Thus, several eruptive pulses of magmatic activity have been established in the complex intrusions of the Kola Belt, including at least four pulses (or phases) in the Fedorovo-Pansky Complex: a 2526-2516 Ma barren pulse and three ore-bearing of 2505-2485, 2470 and 2450 Ma. For similar intrusions of the Fenno-Karelian Belt, for example, the Penikat intrusion in Finland, five magmatic pulses varying only in geochemistry have been distinguished from the same deep chamber (Iljina \& Hanski 2005).

A total duration for magmatic processes of over $130 \mathrm{Ma}$ in the Kola Belt intrusions is unexpected for many researchers. The multi-phase magmatic duration of the Fenno-Karelian Belt intrusions was short-term and took place about 2.44 Ga years ago. However, there are only a few $\mathrm{U}-\mathrm{Pb}$ precise age estimations for the Fenno-Karelian Belt intrusions (Iljina \& Hanski 
2005). A joint Russian- Finnish research collaboration intended for dating the intrusions of the both belts has recently been initiated. It is expected that the research will result in updating the knowledge about the timing and duration of the Palaeoproterozoic ore-forming intrusions on the Baltic Shield.

The Kola results underline that the layering of the intrusions with thinly-differentiated horizons and PGE reefs was not contemporaneous (or syngenetic), with each intrusion defining its own metallogentic trends in time and space.

\subsection{Metallogenic implications}

The Palaeoproterozoic magmatic activity in the eastern Baltic Shield is associated with the formation of widespread ore deposits: $\mathrm{Cu}-\mathrm{Ni}( \pm \mathrm{PGE}), \mathrm{Pt}-\mathrm{Pd}(. \mathrm{Rh}, \pm \mathrm{Cu}, \mathrm{Ni}, \mathrm{Au}), \mathrm{Cr}, \mathrm{Ti}-\mathrm{V}$ (Mitrofanov \& Golubev 2008; Richardson \& Shirey 2008).

On the Kola Peninsula, economic Cu-Ni (+PGE) deposits are known in the Monchegorsk (c. $2500 \mathrm{Ma}$ ) and Pechenga (c. $1980 \mathrm{Ma}$ ) type intrusions. In the Monchepluton (the Monchegorsk type), syngenetic disseminated $\mathrm{Cu}-\mathrm{Ni}$ (+PGE) ore bodies of magmatic origin are confined to basal parts of magmatic chambers (Papunen \& Gorbunov 1985), while massive rich redeposited ores in the veined bodies of the Monchepluton bottom as well as beyond it (offset bodies) also contain a relatively high portion of platinum among PGE. They are associated mainly with c. 2500 Ma magnesium-rich mafic-ultramafic rocks with $1 \mathrm{Nd}(2.5 \mathrm{Ga})$ values varying from 21 to 22. In comparison, $\mathrm{Cu}-\mathrm{Ni}( \pm \mathrm{PGE})$ ores of the Pechenga type intrusions, that are not discussed, are related to the $1980 \mathrm{Ma}$ gabbro-wehrlite rocks with $1 \mathrm{Nd}(1.98 \mathrm{Ga})$ values varying from+1 to+3 (Hanski et al. 1990; Mitrofanov \& Golubev 2008). The basal ores of the Fedorovo deposit are first of all valuable for platinum-group elements ( $\mathrm{Pt}, \mathrm{Pd}, \mathrm{Rh})$, but nickel, copper and gold are also of economic importance here (Schissel et al. 2002). The ore-forming magmatic and post-magmatic processes are closely related to the Taxitic Zone gabbronorite of $2485 \pm 9$ Ma magmatic pulse.

Pt-Pd $( \pm \mathrm{Cu}, \mathrm{Ni}, \mathrm{Rh}, \mathrm{Au})$ reef-type deposits and ore occurrences of the Vurechuaivench Foothills (Monchepluton) and Western Pansky Block (Fedorovo-Pansky Complex) seem, in terms of genesis, to be associated with pegmatoid leucogabbro and anorthosite rocks enriched in late-stage fluids. Portions of this magma produce additional injections of c. $2500 \mathrm{Ma}$ (Vurechuaivench), c. $2470 \mathrm{Ma}$ (the Lower, Northern PGE reef) and c. $2450 \mathrm{Ma}$ (the Upper, Southern PGE reef of the Western Pansky Block and PGE-bearing mineralization of the Mt Generalskaya intrusion). These nonsimultaneous injections are quite close in terms of composition, prevalence of $\mathrm{Pd}$ over $\mathrm{Pt}$, ore mineral composition (Mitrofanov et al. 2005), and isotope geochemistry of $\mathrm{Sm}-\mathrm{Nd}$ and $\mathrm{Rb}-\mathrm{Sr}$ systems. The $\varepsilon_{\mathrm{Nd}}$ values for the rocks under consideration vary from 21 to 23, which probably indicates a single long-lived magmatic hearth.

Chromium concentration (.1000 ppm) is a typical geochemical feature of the lower maficultramafic rocks of the layered intrusions of the Baltic Shield (Alapieti 1982; Iljina \& Hanski 2005). The chromite mineralization is known in the basal series of the Monchepluton, Fedorovo-Pansky Complex, Imandra lopolith (Russia), Penikat and Narkaus intrusions (Finland) and in chromite deposits of the Kemi intrusion (Finland) and Dunite Block (Monchepluton, 
Russia). On the contrary, Fe-Ti-V mineralization of the Mustavaara intrusion (Finland) tends to most leucocratic parts of the layered series, and to leucogabbro-anorthosite and gabbrodiorite of the Imandra lopolith (Russia) and Koillismaa Complex (Finland).

Thus, PGE-bearing deposits of the region are represented by two types: the basal and the reeflike ones. According to modern economic estimations, the basal type of deposits is nowadays more preferable for mining, even if the PGE concentration (1-3 ppm) is lower compared to the reef-type deposits ( $>5 \mathrm{ppm})$. Basal deposits are thicker and contain more platinum, copper and, especially, nickel. These deposits are accessible to open pit mining.

\subsection{Petrological and geodynamic implications}

Magmatic processes since the Palaeoproterozoic (2.53 Ga) have affected almost the whole region of the East Scandinavian (Kola-Lapland-Karelian) province and a mature continental crust formed (2.55 Ga) in the Neoarchaean (Gorbatschev \& Bogdanova 1993). Thick (up to 3 $\mathrm{km}$ ) basaltic volcanites of the Sumian age (2.53-2.40 Ga) in Karelia, Kola and NE Finland cover an area of $>200000 \mathrm{~km}^{2}$. In the north, magmatic analogues of these volcanic rocks are represented by two belts of layered intrusions and numerous dyke swarms (Vuollo et al. 2002; Vuollo \& Huhma 2005). This together composes a single time-and space-related megacyclic association, the East Scandinavian Large Igneous Province (LIP). All the magmatic units of the province covering a huge area show similar geological, compositional and metallogenic features.

Regional geological settings indicate anorogenic rift-like intraplate arrangements involving volcanoplutonic belts connecting different domains of the Palaeoarchaean Kola-LaplandKarelia protocontinent. This resembles early advection extensional geodynamics of passive rifting that is typical of intraplate plume processes (Pirajno 2007). Geochemical and isotopegeochemical data shed light on features of deep magma source for the LIP rocks. TDM values (Faure 1986) are approximately the age of the depleted mantle reservoir (DM) with slightly enriched Sm-Nd ratios. The TDM values lie within the interval of 3.1-2.8 Ga. The $\varepsilon_{\mathrm{Nd}}$ values vary from-2.4 to +1.2 and similar $\mathrm{I}_{\mathrm{Sr}}$ values (0.703-0.704) obtained for discrete layered intrusions form a narrow range of enriched compositions. It is difficult to argue for a local crustal contamination and we suggest that the magmas producing different rocks of the LIP layered intrusions were derived from a single homogenous mantle source enriched both with typically magmatic ore elements (Ni, TI, V and Pt) and lithophile elements including light REE. To some extent, this reservoir is comparable with the modern EM-1 source (Hofmann 1997).

Isotope ${ }^{4} \mathrm{He} /{ }^{3} \mathrm{He}$ ratio is also a reliable isotope tracer of mantle plume processes (Tolstikhin \& Marty 1998; Bayanova et al. 2006; Pirajno 2007). Their use in studying Precambrian rocks requires special care. Table 10 shows recent helium isotope data for the rocks and minerals of the Kola Belt intrusions. The data indicate that the ${ }^{4} \mathrm{He} /{ }^{3} \mathrm{He}$ isotope ratios of $n \times 10^{6-5}$ correspond to those of the upper mantle and differ from those of the crust $\left(\mathrm{n} \times 10^{8}\right)$ and lower mantle $(\mathrm{n} \times$ $10^{4}$ ) (Tolstikhin \& Marty 1998). The helium isotope data tend to favour a source dominated by mantle-derived magmas with only local crustal contamination. 
According to the available data (Campbell 2001; Condie 2001; Vuollo et al. 2002; Bleeker 2003; Ernst \& Buchan 2003; present study), the peak of the mafic-ultramafic magmatic activity of the Kola-Karelian, Superior and Wyoming provinces has been estimated at c. 2.45 Ga. Fig. 12 presents an attempt to demonstrate some reconstruction of the Archaean supercontinent embodying these three provinces of Europe and North America (Heaman 1997; Bleeker, Ernst, 2006). Insert (A) shows trends of the Kola and Fenno-Karelian Belts of 2.52-2.40 Ga layered intrusions with the intraplate nature interpreted from the results of the present study.

The LIP layered intrusions are directly related to the Baltic Shield metallogeny (Mitrofanov \& Golubev 2008). The 130 Ma duration and multiphase history of the Kola Belt layered mafic intrusions (i.e. 2.53-2.40 Ga) has been shown here. It has also been underlined that the younger intrusions of the Fenno-Karelian Belt clustre at 2.44 Ga (Iljina \& Hanski 2005). The partially asynchronous evolution of these two belts, that are thought to be arms of a mantle plume, is now being examined in more detail as a follow-up to this study within the framework of Russian-Finnish research collaboration.

5. Relationship of Pt-Pd provinces with large igneous provinces, LIP (hot plume fields)

Large igneous provinces (or LIPs, by Campbell, Griffiths, 1990) as derivatives of deep mantle plume or asthenospheric upwelling processes were minutely discussed in May 2006 in China at the International Continental Volcanism Conference (Yi-gang Xu, 2007). A special LIP group along with alkaline, komatiite, felsic ones, is represented by mafic intraplate continental provinces (or mafic LIPs, by Bleeker, Ernst, 2006) composed of thick riftogene sedimentary and volcanic rocks cogenetic with dike swarms and mafic-ultramamfic intrusions.

Grachyov (2003), Pirajno (2007), Bogatikov et al. (2010) cite main geological, geophysical, and geochemical features of geological processes within LIPs related with deep mantle plumes. Taking into account experience of studying ancient (Precambian) areas where most geological and geophysical features of geological units, bodies, and rock compositions fail to be preserved, Felix P. Mitrofanov proposed the following indicators of various rank for intraplate mafic LIPs:

- presence of gravitational anomalies caused by a crust-mantle layer in the crust bottom;

- riftogene (anorogenic) structural ensemble with manifestations of multipath fault tension tectonics identified by the distribution of grabens and volcanic belts, elongated dike swarms, and radial belts of intrusions;

- long duration, polystage and pulsating nature of tectonics and magmatism, continental discontinuities and erosion with early stages of tholeiite-basalt (trappean), boninite-like and subalkaline magmatism in the continental crust, and possible closing stages of the Red Sea spreading magmatism;

- intrusive sills, lopoliths, sheet-like bodies, large dikes and dike swarms. The intrusions are often layered, being different from rocks typical of subduction and spreading zones in terms of nature (Bleeker, Ernst, 2006), with trends of thin differentiation (or layering), with limited development of intermediate and felsic rocks, often with leucogabbro and anorthosite ends and abundant pegmatoid mafic varieties; 
- typical mantle geochemistry of rocks and ores, isotope mantle tracers: ${ }^{143} \mathrm{Nd} /{ }^{144} \mathrm{Nd},{ }^{87} \mathrm{Sr} /{ }^{86} \mathrm{Sr}$, ${ }^{187} \mathrm{Os} /{ }^{188} \mathrm{Os},{ }^{3} \mathrm{He} /{ }^{4} \mathrm{He}$;

- mafic intracontinental LIPs accoumodate large orthomagmatic $\mathrm{Cr}, \mathrm{Ni}, \mathrm{Cu}, \mathrm{Co}, \mathrm{PGE}( \pm \mathrm{Au})$, Ti, V deposits.

The Palaeoproterozoic East-Scandinavian Large Igneous Province (LIP) with a modern area of ca. 1,000,000 $\mathrm{km}^{2}$ occupies the eastern part of the Baltic (or Fennoscandinavian) Shield which basement is represented by the mature Archaean granulite and gneiss-migmatite crust formed $>2550$ Ma. Main features of the structure and description of coumercial Pt-Pd and Cu-Ni-PGE deposits are given in modern publications of F. Mitrofanov, Ye. Sharkov, V. Smolkin, A. Korchagin, T. Bayanova, S. Turchenko, etc. It is worth noting certain preserved geological and geophysical features of this ancient (Palaeoproterozoic) ore-bearing mafic LIP.

Geophysical survey demonstrated the lower part of the Earth crust in the eastern part of the shield to be represented by a transitional crust-mantle layer $(\mathrm{V} p=7.7-7.1 \mathrm{~km} / \mathrm{s})$. Deep xenoliths in the Kandalaksha explosion pipes elevated from this layer have the compositions of granulite and garnet anorthosite with an age of 2460 Ma typical of most bodies in the province (Verba et al., 2005). This shows that masses of deep matter arose not only in the form of volcanic, dikes, and intrusions, but also emplaced to the crust bottom in the course of vast underplating (Mitrofanov, 2010). The outcropped part of the province continues under the platform cover in the northern part of the Russian platform in the form of vast Palaeoproterozoic Baltic-Central Russian wide arc, or intracontinental orogen (Mints, 2011). This certainly expands long-term coumercial opportunities of the province.

Anorogenic autonomous pattern of grabens, dike swarms and belts (rays) of intrusive bodies independent from the structure of the enclosing Archaean gneiss-migmatite frame, is prominent in the Geological Map of the Fennoscandian Shield (2005). The studied intrusions with deposits and prospects compose elongated belts (rays), e.g. northwesttrending Kola belt in the northern part of the province, and northeasttrending Fenno-Karelian belt with the concentration of intrusions in the well-known Monchegorsk ore node (Bayanova et al., 2009).

In the Early Palaeoproterozoic (2530-2400 Ma) epoch of the long history of the LIP evolution, a few stages separated by breaks (conglomerates) sedimentation and magmatism have been distinguished. The Sumi (2550-2400 Ma) stage was principle in the metallogeny of Pt-Pd ores related to the intrusive siliceous highly $\mathrm{Mg}$ boninite-like and anorthositic magmatism (Mitrofanov, 2005; Sharkov, 2006). Such ore-bearing intrusions formed earlier in the Kola belt (Fedorov-Pana and other intrusions: 2530-2450 Ma) and later in the FennoKarelian belt (2450-2400 Ma), according to (Bayanova et al., 2009; Mitrofanov et al., 2012; Ekimova et al., 2011).

A number of new $\mathrm{U}-\mathrm{Pb}$ and $\mathrm{Sm}-\mathrm{Nd}$ isotope data were obtained for various rocks of the mafic layered intrusions of the Kola Belt (Baltic Shield), including those which bear PGE, $\mathrm{Ni}-\mathrm{Cu}$ and $\mathrm{Ti}-\mathrm{V}$ mineralization. A surprisingly long period of multiphase magmatic activity, from 2530-2400 Ma (about $130 \mathrm{Ma}$ ), resulted in the intrusion of large-scale ore-bearing 
intrusions of the Kola Belt. Magmatism continued until about $2400 \mathrm{Ma}$ and generated widespread dykes and small-scale intrusions. These results contrast with the published data, indicating short-term evolution interval (c. $2440 \mathrm{Ma}$ ) for similar intrusions of the FennoKarelian Belt (Iljina \& Hanski 2005).

The two belts of mafic layered intrusions of the Baltic Shield (the Kola and Fenno-Karelian belts), together with the surrounding volcanic rocks and dyke swarms, compose the Palaeoproterozoic East Scandinavian Large Igneous Province (LIP) with an area of $>200000$ $\mathrm{km}^{2}$. The petrologicalgeodynamic interpretation proposed by this chapter of the LIP is a product of a vast longlived plume is based on the homogenous and enriched isotope characteristics of the magmas and also the large volume and widespread distribution of the magmas. It is quite possible, and fully consistent with our observations, that the geochemical signatures of the LIP magmas may well have been in part inherited from the subcontinental lithosphere, as described recently based on Osisotope characteristics for the Bushveld magmas (Richardson \& Shirey 2008).

\section{Acknowledgements}

We thank to D.Wasserburg for ${ }^{205} \mathrm{~Pb}$ artificial spike, J. Ludden for 91500 and Temora standards, F. Corfu, V. Todt and U. Poller for assistance in the establishing of the U-Pb method for single zircon and baddeleyite grains.

The authors would like to thank the following: L. Koval for baddeleyite, zircon, rock-forming and sulphides minerals separation from rock samples; L. Laylina for baddeleyite and zircon analyses using a Cameca MS-46 and for taking images of baddeleyite crystals; N. Levkovich for the chromatographic separation of $\mathrm{U}$ and $\mathrm{Pb}$ for analyses by mass spectrometry at the Geological Institute, Kola Science Center, Russian Academy of Sciences.

The studies were supported by the Russian Foundation of Basic researches (RFBR), projects no. 13-05-00493, OFI-M-13-05-12055, IGCP-SIDA-599 and Program Department of Earth Sciences RAS no. 2,4 .

\section{Author details}

T. Bayanova, F. Mitrofanov, P. Serov, L. Nerovich, N. Yekimova, E. Nitkina and I. Kamensky

*Address all correspondence to: tamara@geoksc.apatity.ru

Geological Institute of the Kola Science Centre, Russian Academy of Sciences (GI KSC RAS), Apatity, Russia 


\section{References}

[1] Alapieti, T.T. 1982. The Koillismaa layered igneous complex, Finland: its structure, mineralogy and geochemistry, with emphasis on the distribution of chromium. Geol. Surv. Finland, Bull. 319, 116.

[2] Alapieti, T.T., Filen, B.A., Lahtinen, J.J., Lavrov, M.M., Smolkin, V.F. \& Voitekhovsky, S.N. 1990. Early Proterozoic layered intrusions in the Northeastern part of the Fennoscandian Shield. Miner. Petrol., 42, 1-22.

[3] Amelin, Yu.V., Heaman, L.M \& Semenov, V.S. 1995. U-Pb geochronology of layered mafic intrusions in the eastern Baltic Shield: implications for the timing and duration of Palaeoproterozoic continental rifting. Precambrian Research, 75, 31-46.

[4] Balashov, Y.A., Bayanova, T.B., \& Mitrofanov, F.P. 1993. Isotope data on the age and genesis of layered basic-ultrabasic intrusions in the Kola Peninsula and northern Karelia, northeastern Baltic Shield. Precambrian Research, 64, N 1/4, 197-205.

[5] Bayanova, T.B. 2004. Age of reference Geological complexes of the Kola region and the duration of igneous processes. Saint-Petersburg, Nauka, 174.

[6] Bayanova, T.B., Nerovich, L.I., Mitrofanov, F.P., Zhavkov, V.A., Serov, P.A. The Monchetundra basic massif of the Kola region: new geological and isotope geochronological data // Doklady Earth Sciences, 2010. V. 431. N 1. C. 288-293.

[7] Bayanova, T., Ludden, J. \& Mitrofanov, F. 2009. Timing and duration of Palaeoproterozoic events producing ore-bearing layered intrusions of the Baltic Shield: metallogenic, petrological and geodynamic implications / In: Reddy S.M., Mazumder R., Evans D.A.D. \& Collins A.S. (eds) // Palaeoproterozoic Supercontinents and Global Evolution. Geological Society, London, Special Publications, 323, 165-198.

[8] Bayanova, T.B, Novikov D.D., Nitkina, E.A., Serov, P.A. \& Mitrofanov, F.P. 2006. Polychronic and long-time interval of the Formation Proterozoic PGE - bearing Fedorovo-Pansky intrusion. Understanding the genesis of ore deposits to meet the demands of the $21^{\text {st }}$ century. $12^{\text {th }}$ quadrennial IAGOD symposium 2006: Abstract. Moscow. (fill №106).

[9] Bayanova, T.B. \& Mitrofanov, F.P. 2005. Layered Proterozoic PGE intrusions in Kola region: new isotope data. $X$ international symposium of platinum "Platinum-Group Elements - from Genesis to Beneficiation and Environmental Impact": Extended abstracts. Oulu, Finland, 289-291.

[10] Bayanova, T.B. 2006. Baddeleyite: A Promising Geochronometer for Alkaline and Basic Magmatism. Petrology. 14, N 2, 187-200.

[11] Bayanova, T.B., \& Balashov, Yu.A 1995. Geochronology of Palaeoproterozoic layered intrusions and volcanites of the Baltic Shield: Proceed. of the 1 st. Intern. Barents 
Sympos. "Geology and minerals in the Barents Region". Norges geol. unders. Spec. Publ. 7, 75-80.

[12] Bayanova, T.B., Galimzyanova, R.M. \& Fedotov, G.A. 2001. Evidence of the multiphase complex history of the Imandra lopolith. Svekalapko. Europrobe project. 6th Workshop. Abstracts. Laumi, Finland. University of Oulu, 7.

[13] Bayanova, T.B., Smolkin, V.F. \& Levkovich, N.V. 1999. U-Pb geochronological study of Mount Generalskaya layered intrusion, northwestern Kola Peninsula, Russia. Transactions of the Institution of Mining and Metallurgy, 108, B83-B90.

[14] Bleeker, W., Ernst, R. 2006. Short-lived mantle generated magmatic events and their dyke swarms: the key unlocking Earth's paleogeographic record back to 2.6 Ga. Dyke Swarms-Time Marker of Crustal Evolution, Balkema Publ. P.1-20.

[15] Bleeker, W. 2003. The late Archaean record: a puzzle in ca. 35 pieces. Lithos. 71, 99-134.

[16] Bogatikov, O.A., Kovalenko, V.I., Sharkov, Ye.V. 2010. Magmatism, tectonics, and geodynamics of the Earth. M., Nauka, 606 p.

[17] Boynton, W.V. 1984. Cosmochemistry of the rare earth elements: meteorite studies / Ed. Henderson P. Rare earth element geochemistry. Amsterdam: Elsevier, 63-114.

[18] Campbell, L.H., Griffiths, R.W. 1990. Implications of mantle plume structure for the evolution of flood basalts. Earth and Planetary Science Letters. V. 99. P. 79-93.

[19] Campbell, I.H. 2001. Identification of ancient mantle plumes. In Ernst R.E. and Buchan (Eds.) Mantle plumes: their identification through time. Geological Society of America, Special Papers. 352, 5-22.

[20] Chashchin, V.V., Bayanova, T.B. \& Apanasevich, E.A. 2002. The Monchegorsk Ore District as an Example of the Palaeoproterozoic Ore-bearing Chamber Structure (Kola, Russia). Geology of Ore Deposits, 44, N 2, 142-149.

[21] Coffin, M.F. \& Eldholm, O. 1994. Large igneous provinces: crustal structure, dimensions and external consequences. Rev. Geophys., 32, 1-36.

[22] Condie K.C. Supercontinents and superplume events: Distinguishing signals in the geology record. Phys. of the Earth and Planetary Interiors, v.146, p.319-332, 2004.

[23] Condie, K.C. 2001. Mantle Plumes and Their Record in Earth History. Condie. Cambridge. University press, 306.

[24] DePaolo, D.J. 1981. Neodymium isotopes in the Colorado Front Range and crastmantle evolution in the Proterozoic / Nature, 291, 21, 193-196.

[25] Dobretsov, N.L. 1997. Permian-Triassic magmatism and sedimentation in Eurasia as a reflection of a superplume. Doklady RAS. V. 354. N 2. P. 220-223. 
[26] Dodin, D.A., Chernyshov, N.M., Cherednikova, O.I. 2001. Russian platinum-group element mineralogy. M., Geoinfor $\mu$ mark. 302 p.

[27] Dyuzhikov, O.A., Distler, V.V., Strunin, B.M., Mkrtshan, A.K., Sherman, M.L., Sluzenikin, S.F., Lurie, A.M. 1988. Geology and ore content of the Norilsk region. M., Nauka. 279 p.

[28] Ekimova, N.A., Serov, P.A., Bayanova, T.B., Elizarova, I.R., Mitrofanov, F.P. 2011. Distribution REE in sulfide minerals and Sm-Nd dating of processes ore forming layered basis intrusions. Doklady RAS. V. 436. N 1. P. 75-78.

[29] Ernst, R.E. \& Buchan, K.L. 2003. Recognizing Mantle Plumes in the Geological Record. Annu. Rev. Earth Planet. Sci., 31, 469-523.

[30] Eules, H.V. \& Cawthorn, R.G. 1995. The Bushveld Complex. Layered Intrusions. Developments in Petrology 15. Amsterdam; N.Y., Tokyo: Elsevier, 181-229.

[31] Faure, G.1986. Principles of Isotope Geology. 2 nd eds.Wiley. New York, 460.

[32] French, I.E., Heaman, L.M., Chacko, T., \& Sristava, R.K. 2008. 1891-1883 Ma Southern Bastar -Cuddapah mafic igneous events, Judia: A newly recognized large igneous province. Precambrian Research, 160, 308-322.

[33] Goldstein, S.J. \& Jacobsen, S.B. 1988. Nd and Sr isotopic systematics of river water suspended material implications for crystal evolution. Earth Plan. Sci. Letters, 87, 249-265.

[34] Gorbatschev, R. \& Bogdanova, S. 1993. Frontiers in the Baltic Shield. Precambrian Research.V. 64, 3-21.

[35] Grachyov, A.F. 2003. Identification of mantle plumes on the basis of studying composition and isotope geochemistry of volcanic rocks. Petrology. V. 11. P. 618-654.

[36] Grochovskaya, T.L., Bakaed, G.F., Sholohnev, A.B., Lapina, M.I., Muravizkaya, G.H., Voitechovich, V.C. 2003. Ore platinometall mineralization in layered Monchegorsk magmatic complex (Kola peninsula, Russia) // Geology of ore deposited, 45, 4, 329-352.

[37] Groshev, N.Yu., Nitkina, Ye.A., Mitrofanov, F.P. 2009. Two-phase mechanism for the generation of PGE mafic rocks in the Fedorovo-Pana intrusion: New geological and isotope-geochemical data. Doklady RAS. V. 427. N 5. P. 669-673.

[38] Groves, D.I., Vielreicher, R.M., Goldfarb, R.J. 2005. Controls on the heterogeneous distribution of mineral deposits through time. Miner. Deposits and Earth Evolution, Geol. Soc. Sp. Publ. V. 248. P. 71-101.

[39] Hanski, E., Huhma, H., Smolkin, V. et al. 1990. The age of the ferropicric volcanics and comagmatic Ni-bearing intrusions at Pechenga, Kola Peninsula, USSR. Bull. Geol. Soc. Finland, 1990, V. 62, No 2, 123-133. 
[40] Hanski, E., Walker, R.J., Huhma, H., \& Suominen, I. 2001. The Os and Nd isotopic systematics of c. $2.44 \mathrm{Ga}$ Akanvaara and Koitelainen mafic layered intrusions in northern Finland. Precambrian Research, 109, 73-102.

[41] Heaman, L.M. \& LeCheminant, A.N. 1993. Paragenesis and U-Pb systematics of baddeleyite (ZrO). Chemical Geology. Elsevier, 110, 95-126.

[42] Heaman, L.M. 1997. Global mafic magmatism at 2.45 Ga: Remnants of an ancient large igneous province? Geology, 25, No 4, 299-302.

[43] Hofmann, A.W. 1997. Mantle geochemistry: the message from oceanic volcanism. Nature, 385, 219-229.

[44] Huhma, H., Clift, R.A., Perttunen, V. \& Sakko, M. 1990. Sm-Nd and Pb isotopic study of mafic rocks associated with early Proterozoic continental rifting: The Perapohja schist belt in Northern Finland. Contrib. Mineral. Petrpol., 104, 369-379.

[45] Iljina, M., \& Hanski, E. 2005. Layered mafic intrusions of the Tornio-Näränkävaara belt. In Lehtinen, M., Nurmi, P.A. \& Rämo, O.T. (Eds.). Precambrian Geology of Finland - Key to the Evolution of the Fennoscandian Shield. Elsevier B.V., Amsterdam. 101-138.

[46] Irvine, T.N. 1982. Terminology for layered intrusions. Journal of Petrology. V. 23, P. 127-162.

[47] Jacobsen, S. B. \& Wasserburg, G. J. 1984. Sm-Nd Isotopic Evolution of Chondrites and Achondrites, II. Earth Planet. Sci. Lett., 67, 137-150.

[48] Korchagin, A.U., Subbotin, V.V., Mitrofanov, F.P., Mineev, S.D. 2009. Kievey PGEbearing deposit in the West Pana layered intrusion. In: Strategic mineral resources of Lapland, Apatity. P. 12-32.

[49] Kramm, U. 1993. Mantle components of carbonatites from the Kola Alkaline Province, Russia and Finland: A Nd-Sr study. Eur J. Mineral, 5, 985-989.

[50] Krogh, T.E. 1973. A Low-Contamination Method for Hydro-thermal Dissolution of Zircon and Extraction of $\mathrm{U}$ and $\mathrm{Pb}$ for Isotopic Age Determinations. Geochim. Cosmochim. Acta, 37, 485-494.

[51] Latypov, R.M., Mitrofanov, F.P., Alapieti, T.T., Halkoaho, A.A. 1999. Petrology of the Lower Layered Horizon, West Pana Tundra. Petrology. V. 7. N 5. P. 509-538.

[52] Latypov, R.M. \& Chistyakova, S.Yu. 2000. Mechanism for differentiation of the Western-Pana layered intrusion. Apatity: Publ. of KSC RAS, 315.

[53] Li, C., Naldrett, A.J. 1993. Sulfide capacity of magma: a quantitative model and its applition to the formation of sulfide ores at Sudbury, Ontario. Economic Geology, 88, 1253-1260. 
[54] Likhachyov, A.P. 2006. Platinum-copper-nickel and platinum deposits. M., Nauka. $496 \mathrm{p}$.

[55] Lubnina, N.V. 2009. East European craton from the Neoarchaean to the Palaeozoic in accordance with palaeomagnetic data. Thesis of a doctorate, M., $41 \mathrm{p}$.

[56] Ludwig, K.R. 1991. ISOPLOT - A plotting and regression program for radiogenic isotope data, version 2.56 // Open-file report 91-445. US Geol. Surv., 40 p.

[57] Ludwig, K.R. 1999. ISOPLOT/Ex - A geochronological toolkit for Microsoft Excel, Version 2.05. Berkeley Geochronology Center Special Publication, 1a, 49.

[58] Ludwig, K.R. 1991. PBDAT - A Computer Program for Processing Pb-U-Th isotope Data. Version 1.22. Open-file report 88-542. US Geol. Surv., 38.

[59] Mints, M.V. 2011. Solid model for the deep structure of the Early Precambrian crust of the East European craton: Palaeogeodynamic consequences. Geotectonics. N 4. P. 3-29.

[60] Mitrofanov, F., Golubev, A. 2008. Russian Fennoscandia metallogeny. Abstr. To 33 IGC. Oslo, Norway.

[61] Mitrofanov, F.P. 2010. Metallogeny of the Kola-Karelian region, Baltic Shield. In: Fundamental geological problems of mineral deposits metallogeny. M., IGEM RAS. P. 257.

[62] Mitrofanov, F.P. 2005. New kinds of minerals resources in the Kola Province: Discoveries and perspectives. In Conference of V.I. Smirnov. M., IGEM RAS. P. 39-53.

[63] Mitrofanov, F.P., Malitch, K.N., Bayanova, T.B., Korchagin, A.U., Zhirov, D.V. Comparison of East-Scandinavian and Norilsk large plume mafic igneous provinces of PGE ores // Proceedings of the MSTU, 2012. V. 15. N 2. P. 380-394.

[64] Mitrofanov, F.P., Zhirov, D.V., Bayanova, T.B. Geological and isotope-geochemical forecast criteria of the PGM and/or Cu-Ni for mafic-ultramafic layered intrusions of the LIPs of Pre-Cambrian Shields // 34th International Geological Congress (IGC), 5-10 August 2012, Brisbane, Australia. SD-ROM-P3M-137 (P. 2300).

[65] Mitrofanov, F.P., Nerovich L.I. 2003. Time of igneous crystallization and metamorphic modifications in the Pyrshin and Abvar autonomous anorthosite intrusions of the Lapland granulite belt. Petrology. V. 11. N 4. P. 381-390.

[66] Mitrofanov, F.P, Smolkin, V.F., Bayanova, T.B, Neradovsky, Yu.N., Ohnenstetter, D., Ohnenstetter, M. \& Ludden, J. 2002. Palaeoproterozoic (2.5-2.4 Ga) Plume Magmatism in the North-Eastern Baltic Shield and Origin of the PGE, Sulphide and Chromite Ore Deposit. Extended abstracts: $9^{\text {th }}$ International Platinum Symposium. Billings, Montana, USA, 309-311. 
[67] Mitrofanov, F.P. \& Bayanova, T.B. 1999. Duration and timing of ore-bearing Palaeoproterozoic intrusions of Kola province. Mineral Deposits: Processes to Processing. Stanley et al. (Eds.). Balkema, Rotterdam, 1275-1278.

[68] Mitrofanov, F.P. \& Nerovich, L.I. 2003. Timming of magmatic crystallization and metamorphic transformation in the Pyrshin and Abvar Autonomous anorthosite massifs, Lapland granulate belt. Petrology. V. 11, No 4, 343-351.

[69] Mitrofanov, F.P., Balabonin, N.L., Bayanova, T.B. et al. 1997. Main results from the study of the Kola PGE-bearing province, Russia. Mineral Deposits. Papunen (Eds.). Balkema, Rotterdam, 483-486.

[70] Mitrofanov, F.P., Balagansky, V.V., Balashov, Yu.A., Gannibal, L.F., Dokuchaeva, V.S., Nerovich, L.I., Radchenko, M.K. \& Ryungenen, G.I. 1993. U-Pb age for gabbroanorthosite of the Kola Peninsula. Doklady RAN, 331, 1, 95-98.

[71] Mitrofanov, F.P., Korchagin, A.U., Dudkin, K.O. \& Rundkvist, T.V. 2005. FedorovoPana layered mafic intrusion (Kola peninsula, Russia): Approaches, methods, and criteria for prospecting PGEs. Exploration for platinum-group elements deposits. Short Course delivered on behalf of the Mineralogical Association of Canada in Oulu, Finland, 35, 343-358.

[72] Naldrett, A.J. 2003. Igneous sulfide deposits of copper-nickel and PGE ores. SPb., SpbGU, 487 p.

[73] Nitkina, E.A. 2006. U-Pb Zircon Dating of Rocks of the Platiniferous Fedorova-Pana Layered Massif, Kola Peninsula. Doklady Earth Sciences, 408, 4, 551-554.

[74] Panteeva, S.V., Gladkochoub, D.P., Donskaya, T.V., Markova, V.V., Sandimirova, G.P. 2003. Determination of 24 trace elements in felsic rocks by inductively coupled plasma mass spectrometry after lithium metaborate fusion // Spectrochimica Acta Part B: Atomic Spectroscopy, 58, 2, 341-350.

[75] Papunen, H. and Gorbunov, G. I. (eds.). 1985. Nickel-Copper deposits of the Baltic Shield and Scandinavian Caledonides. Geological Survey of Finland, Bulletin 333, 394.

[76] Pirajno, F. 2007. Mantle plumes, associated intraplate tectono-magmatic processes and ore systems. Episodes, 30, 1, 6-19.

[77] Reischmann, T. 1995. Precise U-Pb age determination with baddeleyite $\left(\mathrm{ZrO}_{2}\right)$, a case study from the Phalaborwa Igneous Complex, South Africa. S. Afr. J. Geol., 1, 1-4.

[78] Richardson, S.H. \& Shirey, S.B. 2008 Continental mantle signature of Bushveld magmas and coeval diamonds, Nature. 453, 910-913.

[79] Robb, L. 2008. Introduction to ore-forming processes. Blackwell Publ., 373 p.

[80] Scharer, U. \& Gower, C.F. 1988. Crustal Evolution in Eastern Labrador: Constraints from Precise U-Pb Ages. Precambrian Research, 38, 405-421. 
[81] Scharer, U., Wilmart, E., \& Duchesne, J.-C. 1996. The Short Duration and Anorogenic Character of Anorthosite Magmatism: U-Pb Dating of the Rogaland Complex, Norway. Earth Planet. Sci. Lett., 139, 335-350.

[82] Schissel, D., Tsvetkov, A.A., Mitrofanov, F.P. \& Korchagin, A.U. 2002. Basal Platinum-Group Element Mineralization in the Fedorov Pansky Layered Mafic Intrusion, Kola Peninsula Russia. Econ. Geol., 97, 1657-1677.

[83] Serov, P.A., Nitkina, E.A., Bayanova, T.B. \& Mitrofanov, F.P. 2007. Comparison of the new data on dating using $\mathrm{U}-\mathrm{Pb}$ and $\mathrm{Sm}-\mathrm{Nd}$ isotope methods of early barren phase rocks and basal ore-hosting rocks of the Pt-bearing Fedorovo-Pansky layered intrusion (Kola peninsula). Doklady Earth Sciences, 415, 3, 1-3.

[84] Sharkov, Ye.V. 2006. Formation of layered intrusions and related mineralization. M., Scientific world, $364 \mathrm{p}$.

[85] Sharkov, E.V., Bogatikov, O.A., Grokhovskaya, T.L., Snyder, G.A., Taylor, L.A. 1995. Petrology and Ni-Cu-Cr-PGE mineralization of the largest mafic pluton in Europe: the early Proterozoic Burakovsky Layered Intrusion, Karelia, Rossia. International Geology Reviev, 37, 509-525.

[86] Skuf'in, P.K. \& Bayanova, T.B. 2006. Early Proterozoic Central-Type Volcano in the Pechenga Structure and Its Relation to the Ore-Bearing Gabbro-Wehrlite Complex of the Kola Peninsula Petrology, 14, 6, 609-627.

[87] Sluzhenikin, S.F., Distler, V.V., Dyuzhikov, O.A., Kravzov, V.F., Kunilov, V.T., Laputina, N.P., Turovzev, D.M. 1994. Low-sulfide PGE mineralization in the Norilsk differentiated intrusions. Geology of ore deposits. V. 36. N 3. P. 195-217.

[88] Smolkin, V.F., Kremenetsky, A.A., Vetrin, V.R. 2009. Geological and geochemical model of the Palaeoproterozoic ore-magmatic systems, Baltic Shield. National Geology, Iss. 3. P. 54-62.

[89] Smolkin, V.F., Fedotov, Zh.A, Neradovsky, Yu.N., Bayanova, T.B. et al. 2004. Layered intrusions of the Monchegorsk ore region: petrology, mineralization, isotope features and deep structure. Mitrofanov, F.P. \& Smolkin, V.F. (Eds.). Part 1. Apatity: publ. by Kola Science Centre RAS, 177 p.

[90] Stacey, J. S. \& Kramers, J. D. 1975. Approximation of Terrestrial Lead Isotope Evolution by a Two-Stage Model. Earth Planet. Sci. Lett., 26, 207-221.

[91] Starostin, V.I., Sorokhtin, O.G. 2010. Recycling origin model for sulfide copper-nickel deposits with PGEs of Norilsk type. Proceedings of the Earth Sciences Division, RANS, Iss. 19. P. 5-10.

[92] Steiger, R.H. \& Jager, E. 1977. Subcoumission on Geo-chronology: Convention on the Use of Decay Constants in Geo- and Cosmochronology. Earth Planet. Sci. Lett., 36, 359-362. 
[93] Tolstikhin, I.N. \& Marty, B. 1998. The evolution of terrestrial volatiles: A view from helium, neon, argon and nitrogen isotope modeling. Chemical Geology, 147, 27-52.

[94] Tolstikhin, I.N., Dokuchaeva, V.S., Kamensky, I.L. \& Amelin, Yu.V. 1992. Juvenile helium in ancient rocks: II. U-He, K-Ar, Sm-Nd, and Rb-Sr systematics in the Monchepluton. ${ }^{3} \mathrm{He} /{ }^{4} \mathrm{He}$ rations frozen in uranium-free ultramafic rocks. Geochim. Cosmochim. Acta, 56, 987-999.

[95] Tuganova, Ye.V. 2000. Formational types, genesis, and emplacement mechanisms for sulfide platinum-copper-nickel and platinum deposits. VSEGEI, $102 \mathrm{p}$.

[96] Verba, M.L., Vinogradov, A.N., Mitrofanov, F.P. 2005. Evolution of the Earth crust and mineragenic potential of the Barents region. Structure of the lithosphere in the Russian part of the Barents region. Petrozavodsk, Karelian Science Centre RAS. P. 256-311.

[97] Vogel, D.C., Vuollo, J.I., Alapieti, T.T. \& James, R.S. 1998. Tectonic, stratigraphic, and geochemical comparison between ca. 2500-2440 Ma mafic igneous events in the Canadian and Fennoscandian Shields. Precambrian Research, 92, 89-116.

[98] Vrevsky, A.B. \& Levchenkov, O.A. 1992. Geological-geochronological scale of the endogenous processes operated within the Precambrian complexes of the central part of the Kola Peninsula. In: Mitrofanov, F.P. \& Bolotov, V.I. (Eds.). Geodynamics and deep structure of the Soviet Baltic Schield. Apatity, 150 p.

[99] Vuollo, J.I. \& Huhma, H. 2005. Palaeoproterozoic mafic dykes in NE Finland. In: Lehtinen, M., Nurmi, P.A. \& Rämö, O.T. (eds.) Precambrian Geology of Finland. Elsevier. B.V. Amsterdam, 195-236.

[100] Vuollo, J.I., Huhma, H., Stepana, V. \& Fedotov, Zh.A. 2002. Geochemistry and Sm$\mathrm{Nd}$ isotope studies of a $2.45 \mathrm{Ga}$ dyke swarm: hints at parental magma compositions and PGE potential to Fennoscandian layered intrusions. In: Boudreau, A. (ed.). $9^{\text {th }}$ Intern. Platinum Symp., 21-25 July, 2002, Billings, Montana USA, 469-470.

[101] Yekimova, N.A., Serov, P.A., Bayanova, T.B., Yelizarova, I.R., Mitrofanov, F.P. 2011. The REE distribution in sulphide minerals and $\mathrm{Sm}-\mathrm{Nd}$ age determination for the oreforming processes in mafic layered intrusions // Doklady RAS, 436, 1, 75-78.

[102] Yi-Gang, Xu. 2007. Mantle plume, large igneous provinces and lithospheric evolution. Episodes. V. 30. No 1, P. 5.

[103] Zhuravlyov, A.Z., Zhuravlyov, D.Z., Kostitsyn, Yu.A., Chernyshov, I.V. 1987. Determination of the Sm-Nd ration for geochronological purposes // Geochemistry, 8, 1115-1129.

[104] Zozulya, D.R., Bayanova, T.B. \& Nelson, E.G. 2005. Geology and Age of the Late Archaean Keivy Alkaline Province, Northeastern Baltic Shield. Geology, 113, 601-608. 

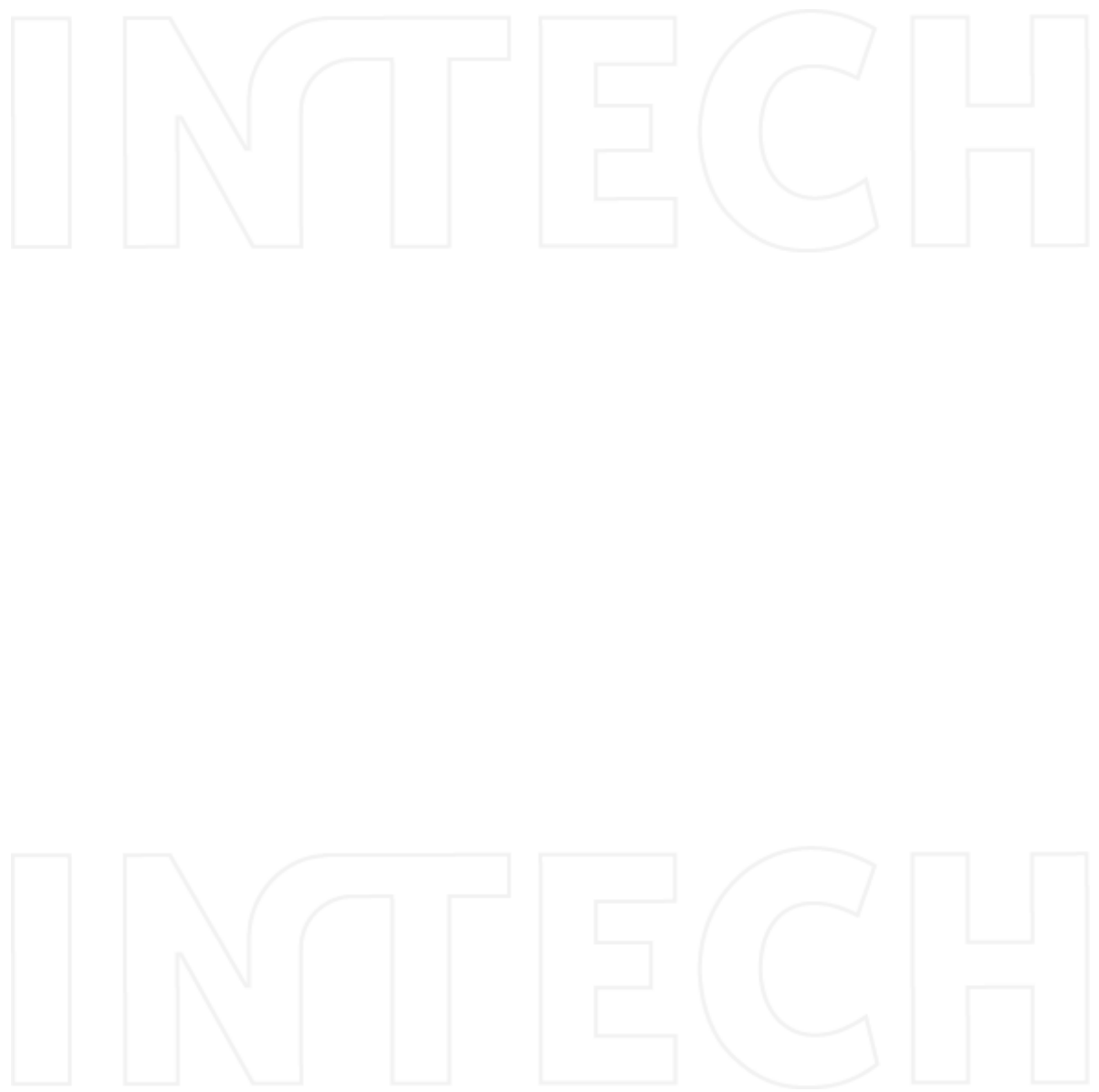